\title{
FACTORS IN THE EFFECTIVENESS OF ANTICIPATORY GUILT AND SHAME APPEALS ON HEALTH COMMUNICATIONS: THE ROLE OF SELF-CONSTRUAL, REGULATORY FOCUS AND PERSONAL CULTURAL ORIENTATION
}

\author{
by \\ Nguyen Hoang Sinh
}

\begin{abstract}
A thesis
submitted to the Victoria University of Wellington in fulfilment of the requirements for the degree of

Doctor of Philosophy in Marketing
\end{abstract}

Victoria University of Wellington 2017 



\begin{abstract}
Negative emotional appeals are commonly used in health messages to cut through the clutter and promote health behaviour change. A research gap exists as to how the emotions of guilt and shame and respective arousals to these emotions act to influence compliance with health messages. Research rarely distinguishes between guilt and shame appeals, different emotional and psychological responses to the two types of appeals, and the main moderators that influence the response to these appeals. To address this gap, this empirical study builds and tests a model for better understanding the processes by which guilt and shame appeals lead to compliance with health messages. Drawing on the theoretical frameworks of cognition, emotion, motivation and research focusing on guilt or/and shame messages and behavioural intention, this study develops an extended model that incorporates influential variables. These include the significant mediating variable of the coping response to emotion, and the moderating variables of self-construal, regulatory focus, and personal cultural orientation.
\end{abstract}

Binge drinking among young adults (aged 16 to 30) is the research context for this study. A series of experiments was conducted to test the research model. Data was collected through an online questionnaire survey among university undergraduates in New Zealand. The main survey collected 301 useable responses including the treatment $(n=266)$ and control (35) groups. The survey data were analysed using a combination of analysis of covariance and covariance-based structural equation modelling. The results broadly support the proposed model for health communications using guilt and shame appeals.

Findings revealed that the coping response has a partial mediating effect on the relationship between guilt/shame arousals and message compliance. Both guilt and shame arousals influence not only message compliance (directly) but also the coping response (indirectly). As predicted, regulatory focus and self-construal were found to moderate guilt/shame arousals from respective emotional appeals. Regulatory focus moderated the levels of shame arousals from shame appeals; that is, prevention-focused individuals exhibited higher shame arousals than their promotion-focused counterparts. Self-construal moderated the levels of guilt arousals from guilt appeals; that is, independent selfconstruals exhibited higher guilt arousals than their interdependent counterparts. However, there were no interactive effects of self-construal with self-referencing or sources of evaluation on guilt/shame arousals. Personal cultural orientation moderated the 
impact of shame arousals, but not those of guilt arousals, on message compliance. That is, shame predicted message compliance in collectivists, but not individualists. Interestingly, there was no main differential effect of guilt versus shame arousals in message compliance, but there was an interactive effect of emotion type with personal cultural orientation as previously mentioned.

The contributions of this study include refining understanding of guilt versus shame, developing the coping response construct, and identifying key moderators and illustrating their impacts on self-conscious emotional arousals. These contributions open new lines of inquiry in the health communications and discrete emotions literature. First, previous discrete emotions literature has mentioned the effects of unintentional emotions, but this research controlled for these effects. It examined guilt and shame separately through respective emotional arousals rather than emotional appeals. Second, the study extended the model of the effectiveness of guilt versus shame appeals in health communications where the coping response is an instrumental mediator. This mediator influences whether or not the receivers actually take on compliant behaviour. Third, the present study differentiated the effect of guilt versus shame appeals. It provides conditions where such appeals are effective. These conditions are type of emotion interacting with self-construal, and regulatory focus. In addition, the study identified the condition under which guilt or shame arousals are most effective. Effectiveness depends on emotion type and personal cultural orientation.

The findings have important practical implications. By understanding how distinct emotion (i.e., guilt versus shame) works and how coping responses (i.e., adaptive versus maladaptive) to these emotions are triggered, practitioners can better structure emotional messaging. Knowledge of message receiver attributes will help them select media appropriately. These attributes are independent versus interdependent, promotion focused versus prevention focused, and individualist versus collectivist. Thus, insights from this research could help health marketers, policy makers as well as health promotion agencies to effectively develop health communications campaigns with more appealing message content and appropriate media selection.

Keywords: Guilt, Shame, Coping response, Self-construal, Regulatory focus, Personal cultural orientation, Binge drinking, Emotional appeal, Health communications. 
To my family,

Vuong Le, Kent, and Huda 


\section{Acknowledgements}

I could not have completed this thesis without the assistance and support of many people. I wish to acknowledge and thank them.

Firstly, I would like to express my sincere gratitude to my supervisors, Assoc. Prof. Daniel Laufer and Dr Jayne Krisjanous, for their guidance, advice, and dedication throughout my $\mathrm{PhD}$ study. Thank you, Dan, for always pushing me like asking for "a justification for every action". Jayne, thank you so much for your continued interest in my work and family as well. It was truly such a motive to lead me through this research work.

I would especially like to thank my grand family for their support and sacrifice throughout the course of my study in New Zealand. Thanks to my parents, especially my mother-in-law, who looked after my family while I was absent from home. Thanks to my wife, Vuong Le, and my sons, Kent and Huda, for love, encouragement, and understanding.

Throughout my PhD journey, there are other people who supported me, to those I need to give a special thanks - Dr James Richard (former PhD Programme Director), Helen Barnard, Kathryn Lawrence (both proof-readers), Linsell Richards, Helena Cook (both former Team Leaders, New Zealand Scholarships Programme, Victoria International), among others.

A special thanks also to the Faculty of Commerce, the School of Marketing and International Business (SMIB) for the support and assistance offered by the academic and administrative staff over the course of the $\mathrm{PhD}$ programme. Especially, my thanks goes to the SMIB Research Committee for the constructive feedback to my PhD proposal and the funding of fieldwork and conference attendance.

My sincere thanks goes to the New Zealand ASEAN Scholar Awards for offering me a full scholarship to pursue a $\mathrm{PhD}$ in Marketing at Victoria University of Wellington in New Zealand.

Thanks to staff and students from Ho Chi Minh City Open University, Ho Chi Minh City University of Social Sciences and Humanities, and Hoa Sen University for assisting me in 
data collection in Vietnam (even though the data was not included in this thesis). Thanks also to all students who were involved in my study both in New Zealand and Vietnam, without your participation the study would not have been possible.

I also acknowledge the examiners, reviewers whose comments and suggestions that have been incorporated into the benefit of this thesis.

I would like to thank my PhD cohorts, past and present, at the SMIB for their friendship, generosity, and cheerfulness. I am particularly grateful to Heath Johnson for proof reading my $\mathrm{PhD}$ proposal.

Finally, thanks to my colleagues, friends, and flatmates whom I have worked, played, and shared with over the past few years. In particular, thanks to members of Vietnamese Tennis Club of Wellington.

They are too numerous to name, but they have my gratitude. 


\section{List of Publications}

Sinh, N. H. (2017). Guilt and Shame Appeals in Health Communications. Paper presented at the Australia and New Zealand Marketing Academy 2017 Conference, Melbourne, Australia.

Sinh, N. H., Laufer, D., \& Krisjanous, J. (2017). The Effectiveness of Guilt and Shame Appeals on Health Communications: The Role of Coping Response, SelfConstrual and Personal Cultural Orientation. Paper presented at the American Academy of Advertising 2017 Global Conference, Tokyo, Japan.

Sinh, N. H. (2017). Factors in the Effectiveness of Guilt and Shame Appeals on Health Communications: The Role of Coping Response, Self-Construal and Personal Cultural Orientation. Paper presented at the Macromarketing Conference 2017 Doctoral Colloquium, Queenstown, New Zealand.

Sinh, N. H. (2015). The Effectiveness of Guilt and Shame Appeals on Health Communications: The Role of Self-Construal, Regulatory Focus and Culture. Proceedings of the International Conference on Business 2015 (pp. 372-388). Ho Chi Minh City: VNU-HCMC Press. 


\section{Table of Contents}

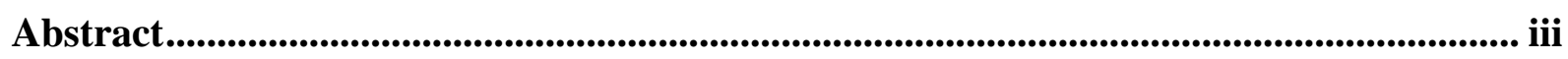

Acknowledgements ..............................................................................................................................................vi

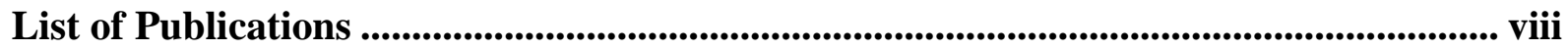

Table of Contents ......................................................................................................................................ix

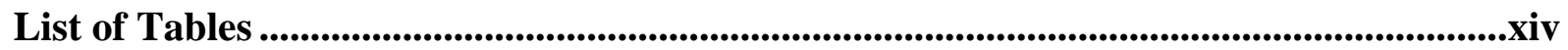

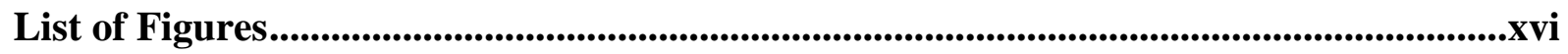

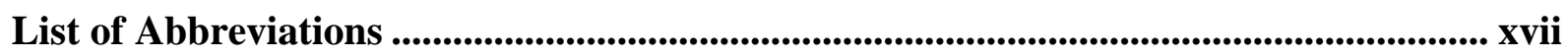

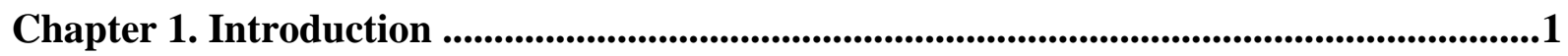

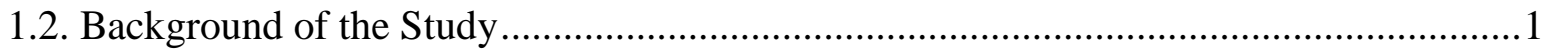

1.2.1. Health Communications Using Guilt and Shame .....................................................2

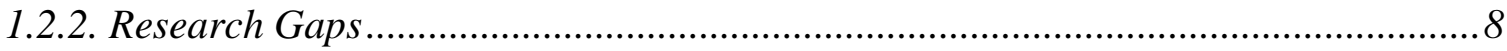

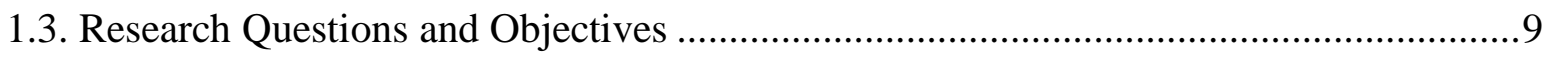

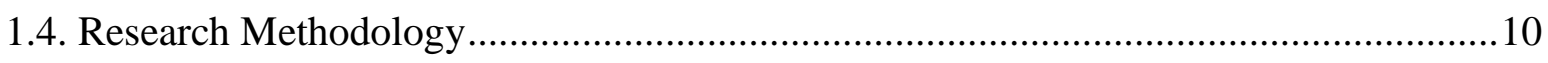

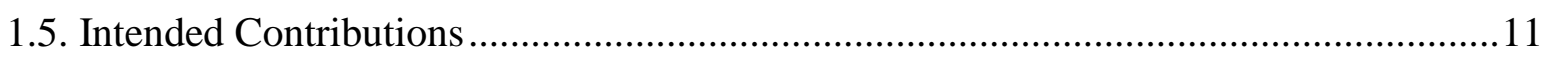

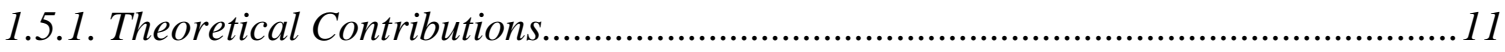

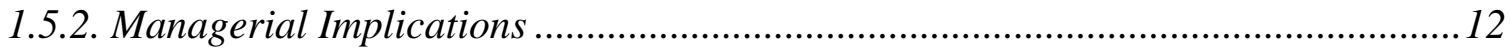

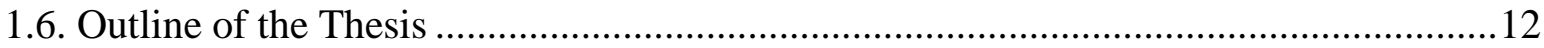

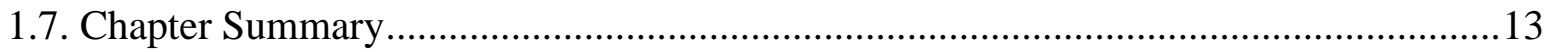

Chapter 2. Literature Review ...................................................................................14

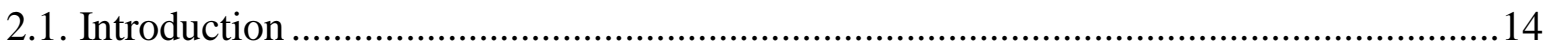

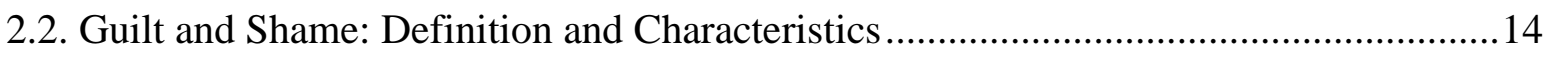

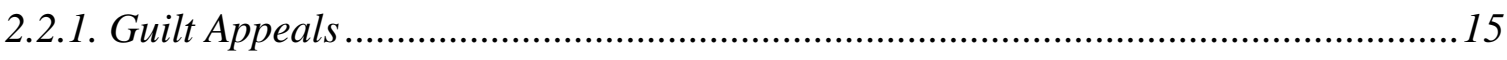

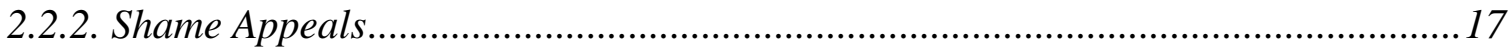

2.2.3. Distinction between Guilt and Shame Appeals..................................................... 19

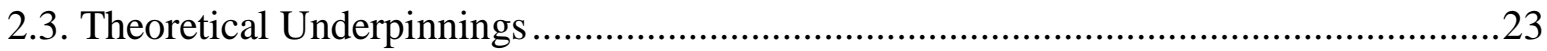

2.3.1. Cognitive-Motivational-Relational Theory of Emotion ...........................................23

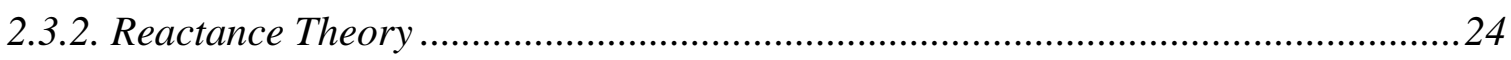

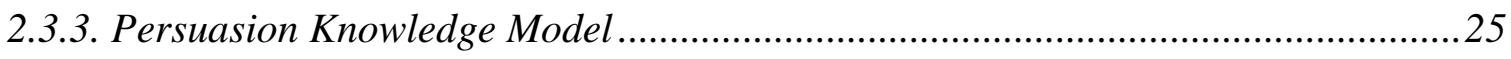

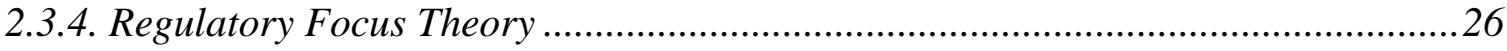

2.4. An Extant Model of the Effectiveness of Guilt Appeals.............................................27

2.5. A Proposed Model of Guilt and Shame Appeals on Health Communications ..............30

2.5.1. Emotional Appeals versus Emotional Arousals.......................................................30 
2.5.2. Coping Responses

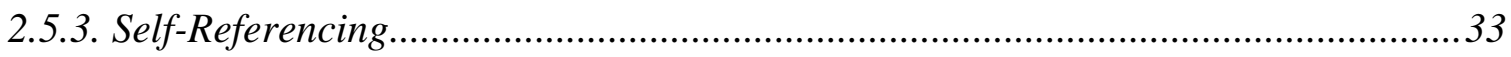

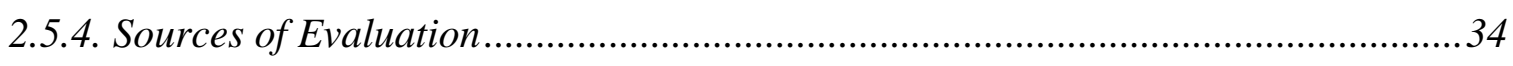

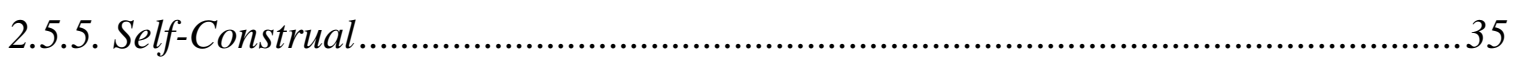

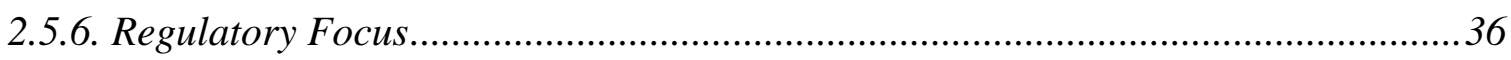

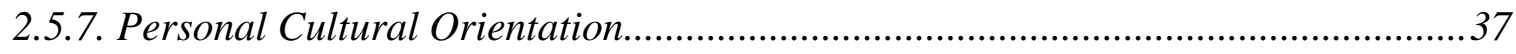

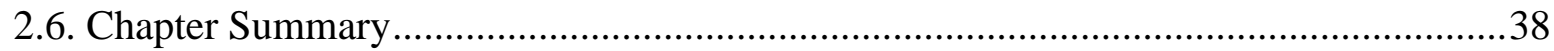

Chapter 3. Conceptual Framework and Hypotheses.......................................................40

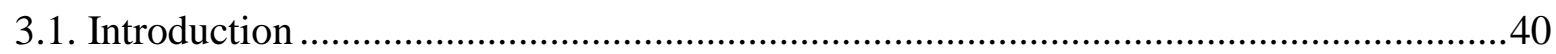

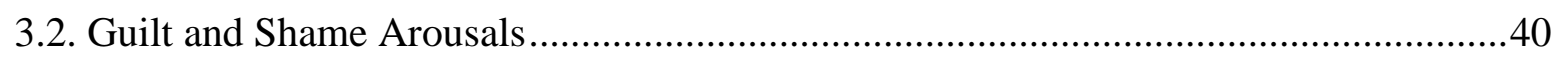

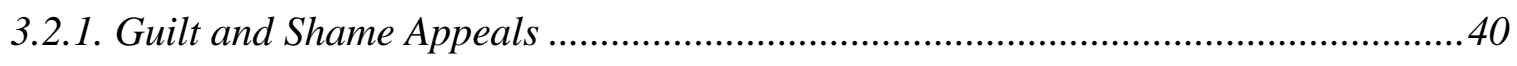

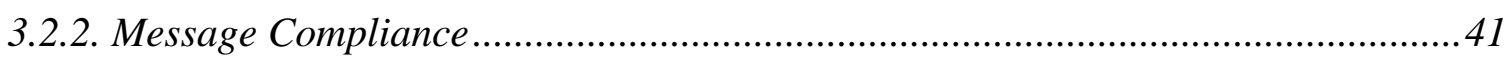

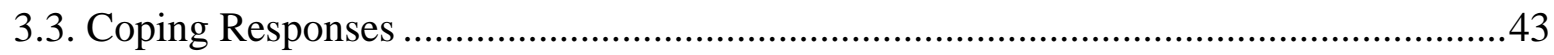

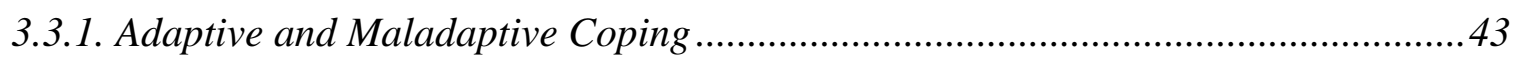

3.3.2. Mediating Emotional Arousal and Message Compliance .......................................4

3.4. Varying Guilt and Shame Arousal Intensity ...........................................................4

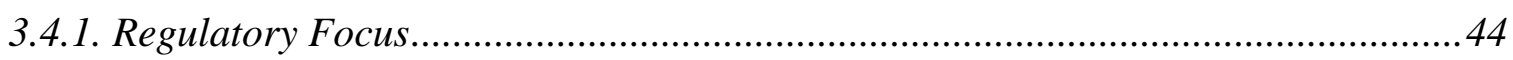

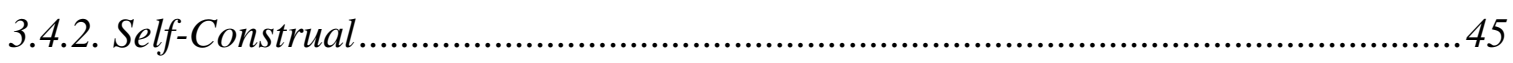

3.4.3. Self-Construal Interacting with Self-Referencing and Sources of Evaluation ........48

3.5. Under Which Personal Cultural Orientation Is Guilt or Shame Most Effective? ..........52

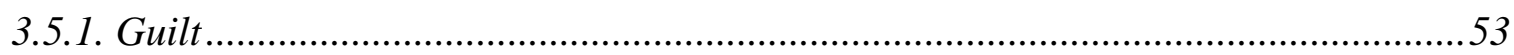

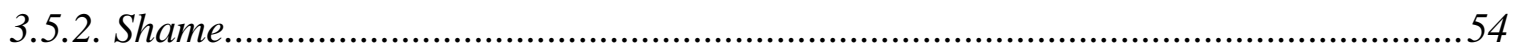

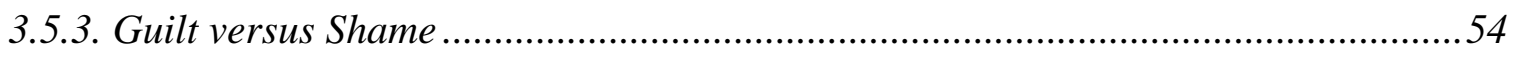

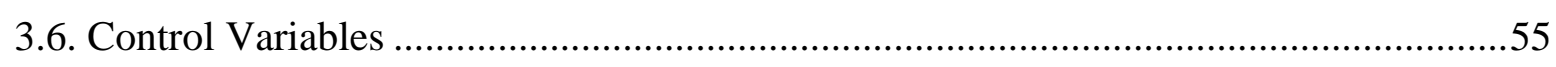

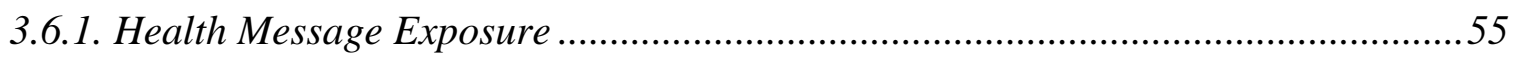

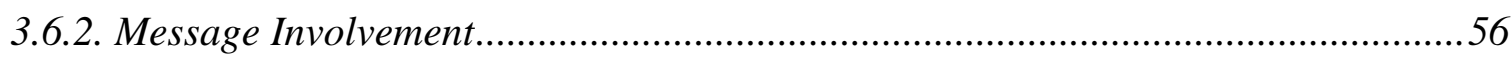

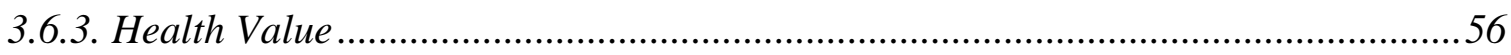

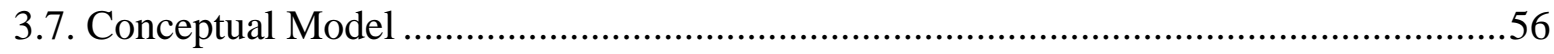

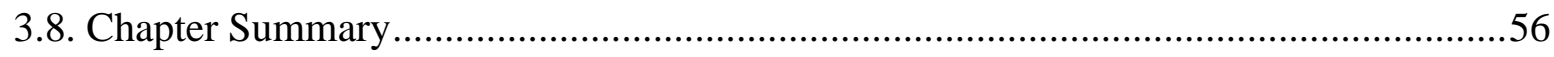

Chapter 4. Research Methodology and Method ................................................................58

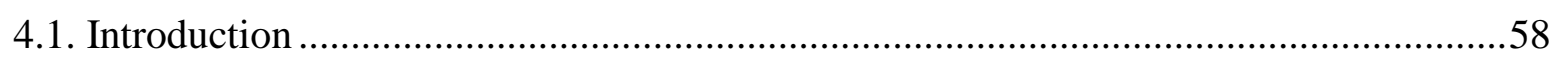

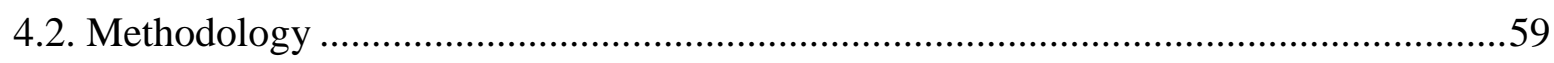

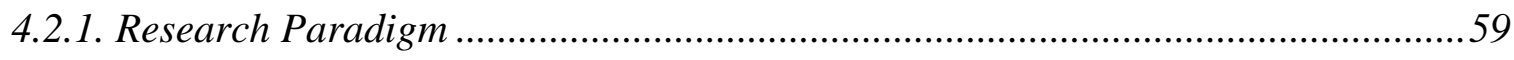

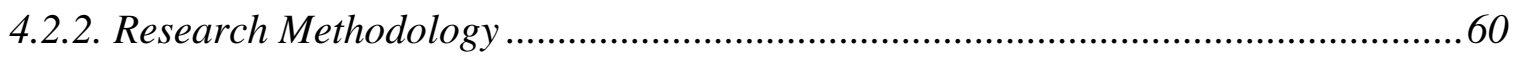


4.2.3. Experiment 62

4.3. Survey Methods .71

4.3.1. Sampling .71

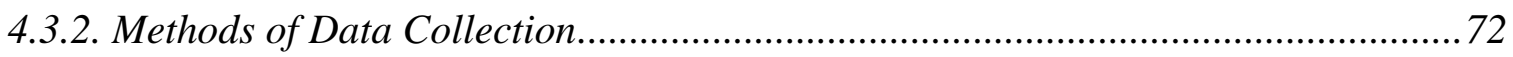

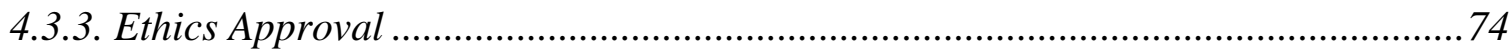

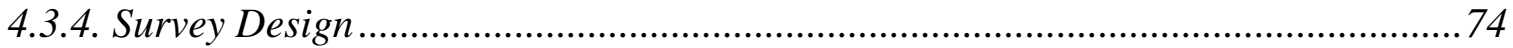

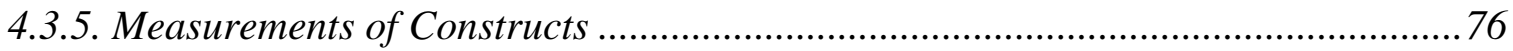

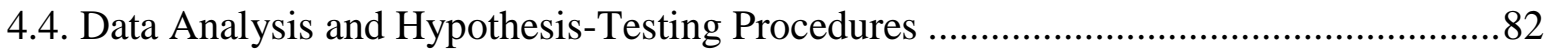

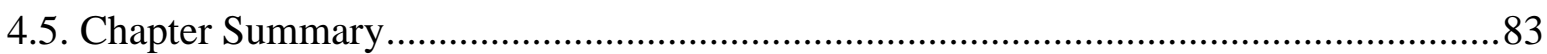

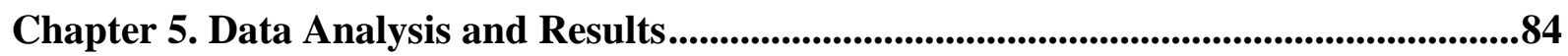

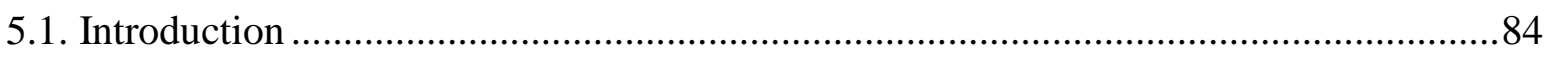

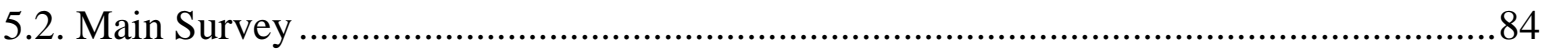

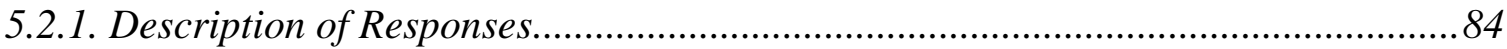

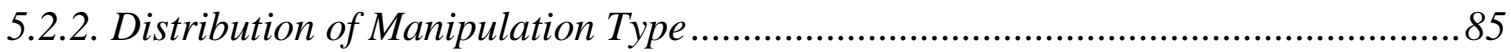

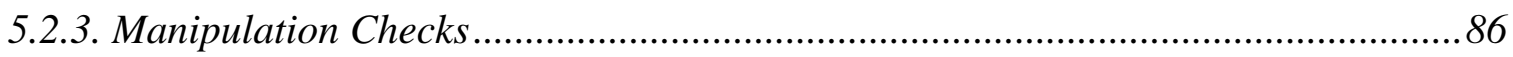

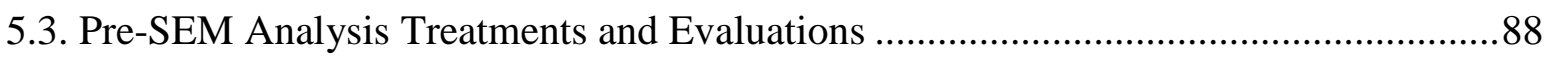

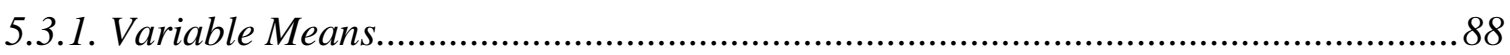

5.3.2. Missing Data and Negative Questions ................................................................ 88

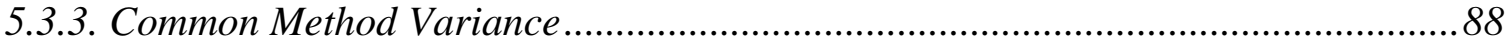

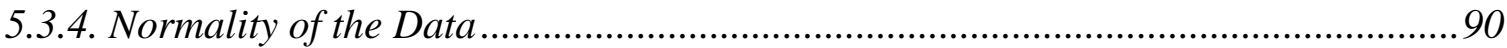

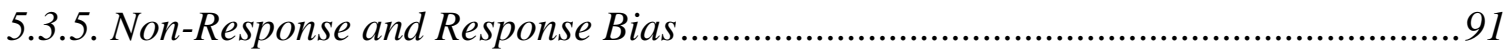

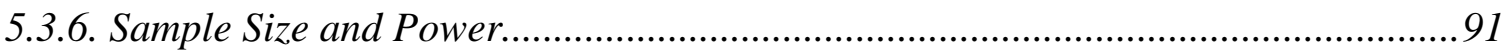

5.4. Critical Statistical Values Applied in the Study ....................................................... 91

5.4.1. Confirmatory Factor Analysis and Model Fit Statistics........................................ 92

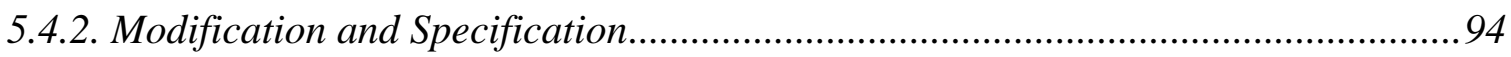

5.5. Confirmatory Factor Analysis and Measurement Model ...........................................94

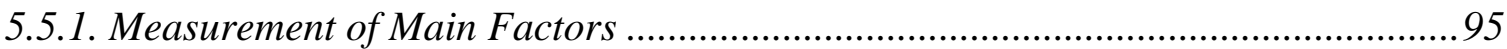

5.5.2. Measurement of Explanatory Factors ................................................................. 98

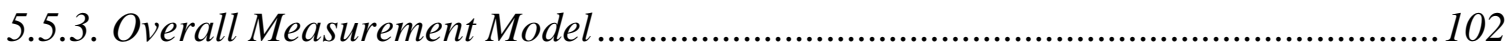

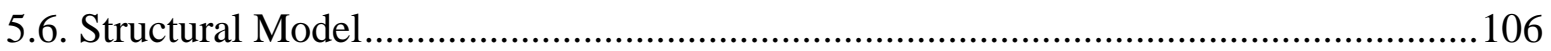

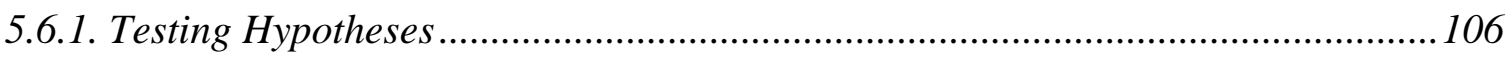

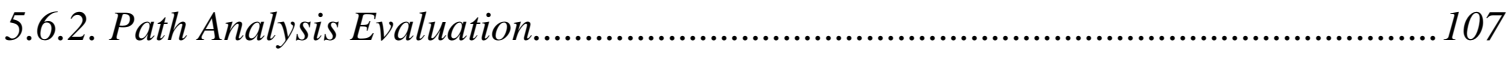

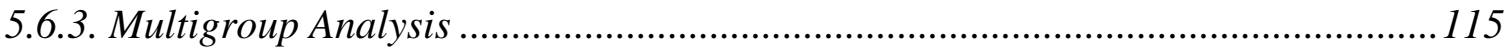

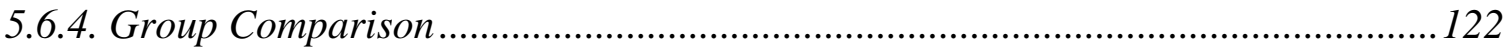


5.6.5. Additional Research Findings

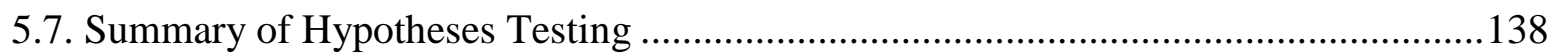

Chapter 6. Discussion of Results.........................................................................142

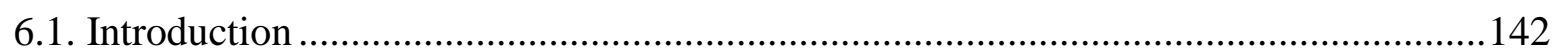

6.2. Effects of Guilt and Shame on Behavioural Intention ...............................................143

6.2.1. Emotional Appeals and Emotional Arousals...................................................... 143

6.2.2. Emotional Arousals and Message Compliance ................................................... 144

6.2.3. Emotional Arousals and Coping Responses....................................................... 146

6.2.4. Coping Responses and Message Compliance ...................................................... 147

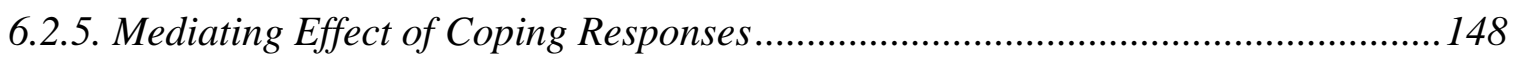

6.2.6. Moderating Effect of Personal Cultural Orientation ........................................... 151

6.2.7. Moderation Effect of Distinct Emotion Type ....................................................... 153

6.3. Moderating Effects of Regulatory Focus and Self-Construal ..................................154

6.3.1. Moderating Effect of Regulatory Focus on Guilt/Shame Arousal........................ 154

6.3.2. Moderating Effect of Self-Construal on Guilt/Shame Arousal..............................157

6.3.3. Interactive Effects of Self-Construal with Self-Referencing, Sources of

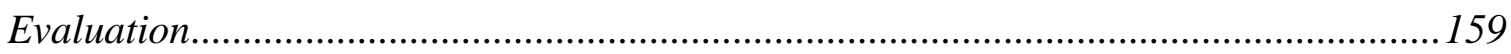

6.4. Further Dependent and Demographic Factors ....................................................... 163

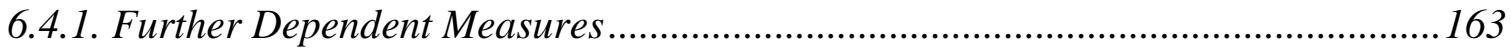

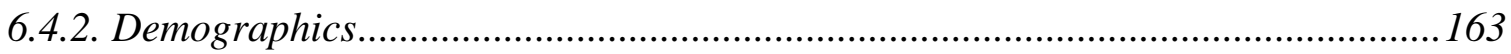

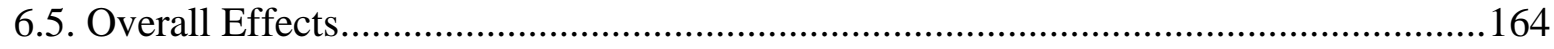

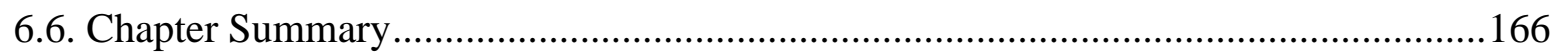

Chapter 7. Contributions, Implications and Conclusion ...............................................168

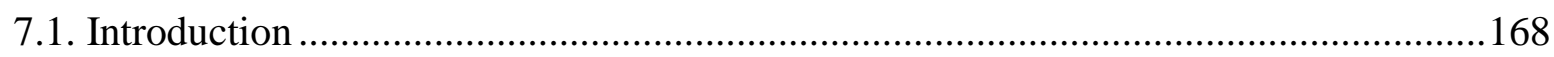

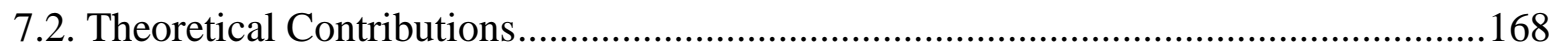

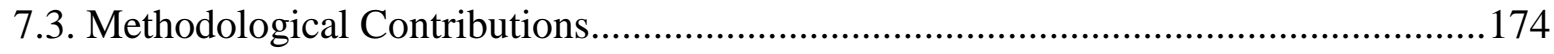

7.3.1. The Development of a New Set of Stimulus Materials..........................................174

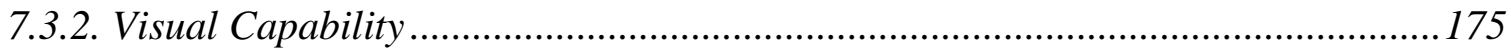

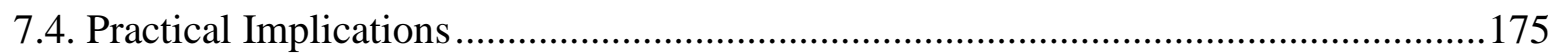

7.5. Limitations and Future Research Directions .......................................................... 178

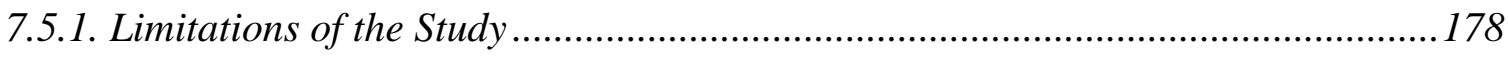

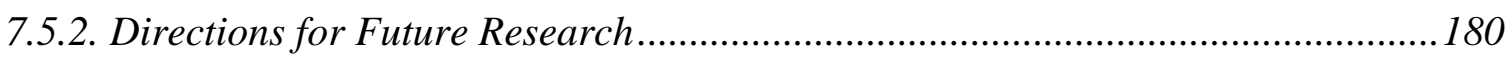

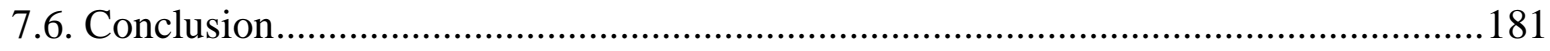


7.6.1. Research Question One: Do Self-Construal, Regulatory Focus and Personal Cultural Orientation Influence the Relative Effectiveness of Guilt and Shame Appeals towards Health Message Compliance?

7.6.2. Research Question Two: Does the Coping Response to Guilt and Shame

Arousals Influence Health Message Compliance? ............................................................ 183

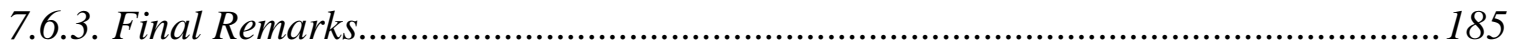

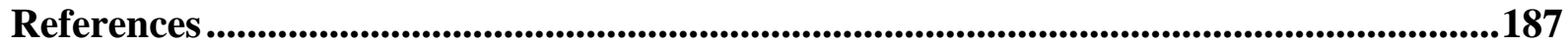

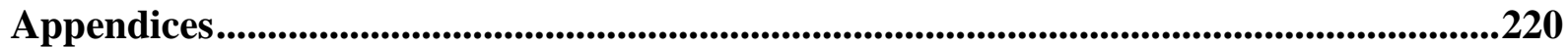

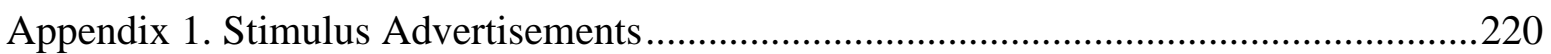

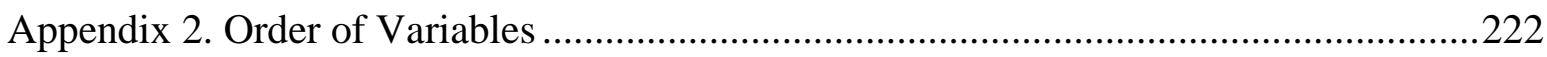

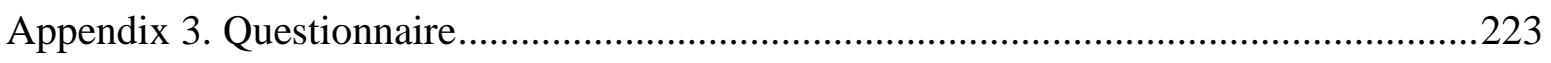

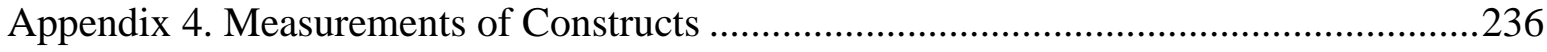

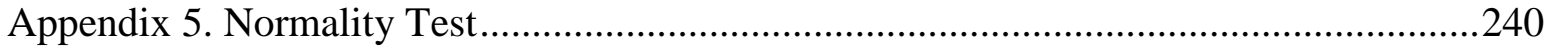

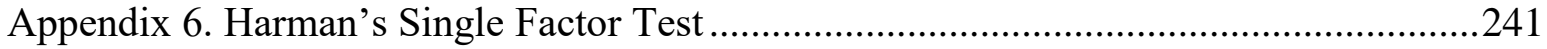

Appendix 7. Marker Variable Correlations ......................................................................241

Appendix 8. Two Waves of Respondents Key Variable Statistics ...................................242

Appendix 9. Items Removed from the Final Model.....................................................243

Appendix 10. Correlation Coefficients in the Modified Measurement Model ..................243

Appendix 11. Final Overall Measurement Model for Guilt ..............................................244

Appendix 12. Final Overall Measurement Model for Shame ….....................................245 


\section{List of Tables}

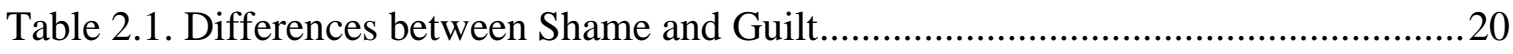

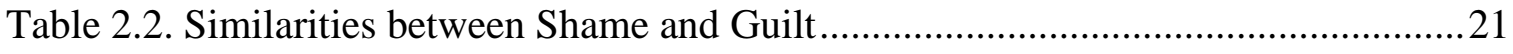

Table 2.3. Health Examples of Guilt and Shame Appeals.............................................22

Table 4.1. Experiment Design: 2 x 2 x 2 between-Subjects ...........................................62

Table 4.2. Emotional Type's Highlights and Consequences of Drinking ........................65

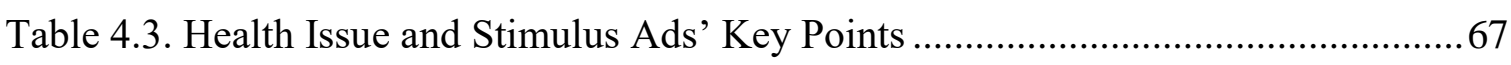

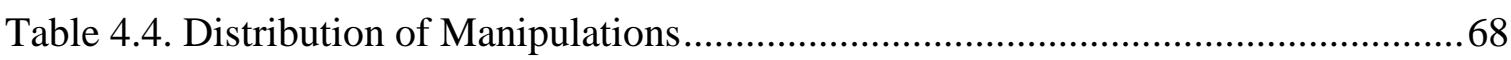

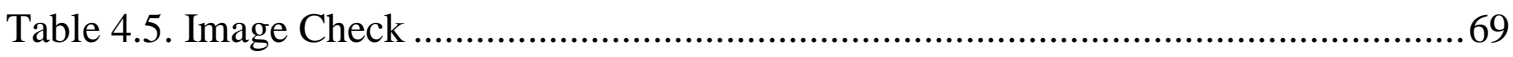

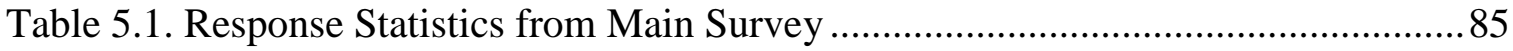

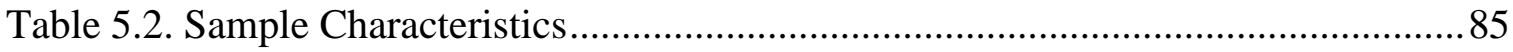

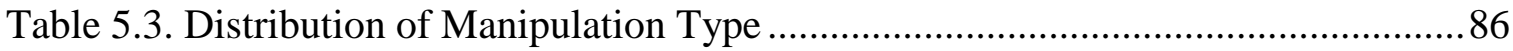

Table 5.4. Descriptive Statistics and Assessment of Normality of the Data .....................90

Table 5.5. Rules of Thumb for CFA and Model Fit Statistics ........................................93

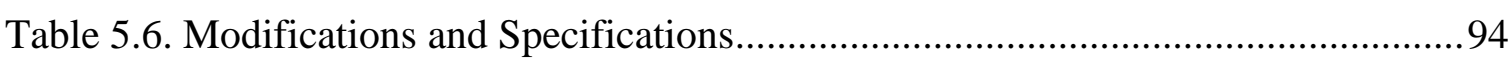

Table 5.7. Model Fit Statistics for the Measurement Model - Guilt ................................96

Table 5.8. Model Fit Statistics for the Measurement Model - Shame .............................. 97

Table 5.9. Model Fit Statistics for the Measurement Model - Explanatory Factors .........99

Table 5.10. Model Fit Statistics for the Final Measurement Model ................................102

Table 5.11. Standardised Factor Loadings, C.R. and AVE Statistics for Convergent

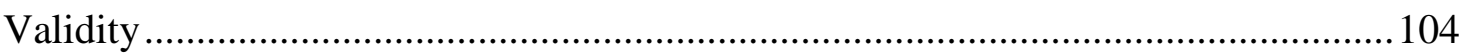

Table 5.12. Discriminant Validity: Fornell-Larcker Criterion....................................... 105

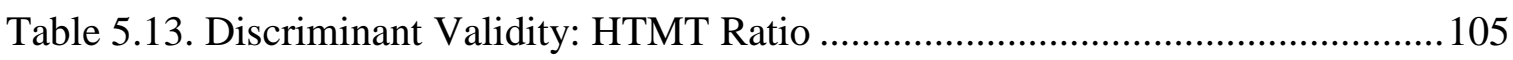

Table 5.14. Structural Model Path Coefficients and Significance Level......................... 107

Table 5.15. Direct and Indirect Effects for Guilt/Shame (bootstrapping $n=2,000 ; \alpha=.05$ )

Table 5.16. Structural Model Path Coefficients and Significance Level with Control ...115

Table 5.17. Measurement Invariance Tests for Guilt - Self-Construal ...........................117

Table 5.18. Measurement Invariance Tests for Shame - Self-Construal ........................118

Table 5.19. Measurement Invariance Tests for Guilt - Regulatory Focus ......................119

Table 5.20. Measurement Invariance Tests for Shame - Regulatory Focus ...................120

Table 5.21. Measurement Invariance Tests for Guilt - Personal Cultural Orientation ... 120

Table 5.22. Measurement Invariance Tests for Shame - Personal Cultural Orientation. 121 
Table 5.23. Group Comparison on the Basis of Regulatory Focus

Table 5.24. Adjusted Means for Regulatory Focus on Guilt/Shame Arousal

Table 5.25. Group Comparison on the Basis of Self-Construal ...................................... 124

Table 5.26. Adjusted Means for Self-Construal on Guilt/Shame Arousal

Table 5.27. Adjusted Means of Self-Construal and Type of Appeal on Guilt/Shame Arousal 126

Table 5.28. Interaction of Self-Construal and Type of Appeal for Guilt 127

Table 5.29. Interaction of Self-Construal and Type of Appeal for Shame 128

Table 5.30. Adjusted Means for Self-Referencing on Guilt/Shame Arousal 129

Table 5.31. Adjusted Means for Source of Evaluation on Guilt/Shame Arousal

Table 5.32. Group Comparison on the Basis of Personal Cultural Orientation 130

Table 5.33. Adjusted Means for Personal Cultural Orientation on Message Compliance 130

Table 5.34. Interaction of Personal Cultural Orientation and Type of Emotion 132

Table 5.35. Level of Emotional Arousal and Type of Coping Response 133

Table 5.36. Mean Message Compliance by Type of Coping Response 134

Table 5.37. Zero-order Pearson Correlations for Dependent Measures 135

Table 5.38. Group Comparison on the Basis of Gender 136

Table 5.39. Adjusted Means for Gender on Message Compliance 137

Table 5.40. Group Comparison on the Basis of Alcohol Use Disorder. 137

Table 5.41. Adjusted Means for AUDIT Level on Message Compliance 138

Table 5.42. Summary of Hypotheses Testing 138

Table 6.1. Zero-Order Pearson Correlations for Key Variables 164

Table 6.2. Path Coefficients across Moderators 165 


\section{List of Figures}

Figure 2.1. Extant Model of the Effectiveness of Guilt Appeals.

Figure 2.2. Proposed Model of the Effectiveness of Guilt and Shame Appeals on Health

Communications. 38

Figure 3.1. Conceptual Model of the Effectiveness of Guilt and Shame Appeals on Health

Communications. .57

Figure 4.1. Outline of Chapter 4 58

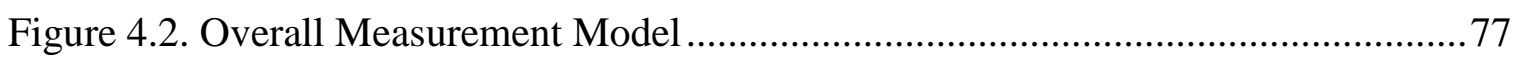

Figure 5.1. Final Measurement Model for Guilt.......................................................... 96

Figure 5.2. Final Measurement Model for Shame ….....................................................98

Figure 5.3. Final Measurement Model for Explanatory Factors......................................99

Figure 5.4. Final Overall Measurement Model................................................................ 103

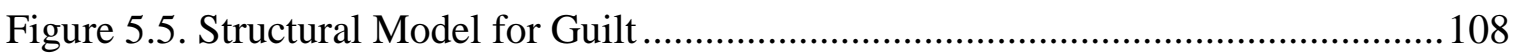

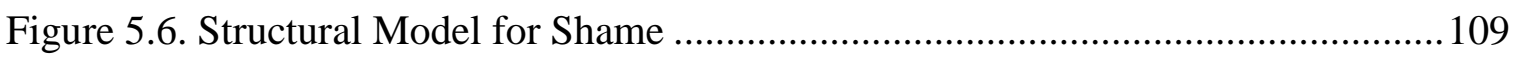

Figure 5.7. Structural Model for Guilt with Control.................................................. 114

Figure 6.1. Direct and Indirect Relationships between Emotional Arousal and Message

Compliance 149

Figure 6.2. Regression of Guilt and Shame Arousal on Message Compliance between Personal Cultural Orientations 152

Figure 6.3. Effect of Regulatory Focus and Type of Emotion on Emotional Arousal ....156

Figure 6.4. Effect of Self-Construal and Type of Emotion on Emotional Arousal .........158

Figure 6.5. Moderating Effects of Regulatory Focus and Self-Construal on Emotional Arousal 162 


\section{List of Abbreviations}

AAt

Ad

AMOS

ANCOVA

ANOVA

AUDIT

AVE

BCCI

BDI

C.R.

c.r.

CFA

CFI

CMV

$\mathrm{CN}$.

ColPCO

CR

DF

EAp

Ear

EFA

GAp

GAr

HME

HMI

HTMT

HV

IndPCO

IndSC

IntSC

LB

MAt
Attitude towards the Advertisement

Advertisement

Analysis of Moment Structures

Analysis of Covariance

Analysis of Variance

Alcohol Use Disorders Identification Test

Average Variance Extracted

Bias-Corrected Confidence Intervals

..Binge Drinking Intention

..Construct Reliability

critical ratio

.Confirmatory Factor Analysis

.Comparative Fit Index

.Common Method Variance

..Critical N

Collectivist

..Coping Response

..Degree of Freedom

..Emotional Appeal

..Emotional Arousal

..Exploratory Factor Analysis

Guilt Appeal

..Guilt Arousal

..Health Message Exposure

..Health Message Involvement

..Heterotrait-Monotrait

..Health Value

.Individualist

..Independent Self-Construal

.Interdependent Self-Construal

..Lower Bound

Attitude towards the Message 


\begin{tabular}{|c|c|}
\hline MC ........ & ...Message Compliance \\
\hline MI ....... & ...Modification Index \\
\hline ML ......... & ....Maximum-Likelihood \\
\hline $\mathrm{PCO} \ldots . .$. & ....Personal Cultural Orientation \\
\hline PreRF ... & ...Prevention Focus \\
\hline ProRF .... & ....Promotion Focus \\
\hline RF........... & ...Regulatory Focus \\
\hline RMSEA & ...Root Mean Square Error of Approximation \\
\hline S.E. ........ & ...Standard Errors \\
\hline S.R. ..... & ..Standardised Residuals \\
\hline SAp .... & ..Shame Appeal \\
\hline SAr ...... & ..Shame Arousal \\
\hline $\mathrm{SC} \ldots \ldots \ldots$ & ..Self-Construal \\
\hline $\mathrm{SD} \ldots \ldots$. & ...Standard Deviations \\
\hline SEM ..... & ...Structural Equation Modelling \\
\hline SPSS ..... & ...Statistical Package for the Social Sciences \\
\hline sRMR ... & ...Standardised Root Mean Square Residual \\
\hline TLI ........ & ...Tucker and Lewis Index \\
\hline UB ..... & ..Upper Bound \\
\hline
\end{tabular}




\section{Chapter 1. Introduction}

This chapter introduces the topic area of the present research. First, the background of the study on health communications using guilt and shame is introduced. Second, the importance of conducting this research is justified, followed by a presentation of the research questions and objectives. Then, the significance of this study for academics and practitioners is outlined. Finally, an outline of the thesis is given.

\subsection{Background of the Study}

Health communications is commonly used in social marketing campaigns for public health (Evans \& McCormack, 2008; Maibach, Abroms, \& Marosits, 2007) and defined as the creating and delivery of messages and strategies to promote the health of individuals and communities (Noble \& Camit, 2005; Roper, 1993). Health communications is the study and use of methods to influence and inform individual as well as community decisions that improve health (Freimuth, Linnan, \& Potter, 2000). Typically, health communications involves the persuasive use of messages and communications strategies (Hill, 2001; Lee \& Kotler, 2016). While it is only one component used by health marketers to develop social marketing campaigns, understanding how health communications messages work is critical for the effectiveness of holistic campaigns. Within messages, emotional appeals are often used in health communications as persuasive devices and to cut through the clutter (Boudewyns, Turner, \& Paquin, 2013; Turner, 2012).

In this regard, this proposed study builds and tests a theoretical model for understanding the processes by which guilt and shame appeals may lead to better compliance with health messages. To date, there is little understanding of how and when guilt and shame appeals in health communications can encourage a desired pro-health or pro-social behaviour. Although the proposed context and goal is preventing binge drinking, it is anticipated that the knowledge gained will be able to be generalised to other health contexts. The present lack of understanding regarding guilt and shame appeals is partly due to a lack of research on the distinction between the two types of appeals, the differing of emotional and psychological responses to them, and the main moderators to the effectiveness of guilt and shame appeals in motivating positive behaviour. Drawing on the existing literature's focus on pro-social and charitable donation messages and 
behavioural intention, this present study builds a theoretical model incorporating a number of variables that to date have generated little attention in previous research in this field. These variables include the mediator of the coping response to emotion, and the moderators of self-construal, regulatory focus, and personal cultural orientation. The findings will inform how these variables are influential in predicting the effectiveness of guilt and shame appeals in promoting healthy and pro-social behaviour. By addressing the current knowledge gaps and informing the development of more effective processes for health communications using guilt and shame appeals, the current research will benefit health marketers, policy makers and health promotion agencies alike.

\subsubsection{Health Communications Using Guilt and Shame}

Message appeals (rational and emotional) are important factors in how messages are attended to and interpreted by a receiver (Flora \& Maibach, 1990; Parrott, 1995). Emotion-based appeals are common (de Hoog, Stroebe, \& de Wit, 2005; Dickinson \& Holmes, 2008; Pechmann, Zhao, Goldberg, \& Reibling, 2003; Schoenbachler \& Whittler, 1996). Negative emotional appeals are most frequently used (Agrawal \& Duhachek, 2010; Keller \& Lehmann, 2008; Keller, Lipkus, \& Rimer, 2003) because they can create an emotional discomfort that can be remedied by engaging in the featured behaviour. Along with fear, guilt and shame appeals may be used in health messages owing to their presumed ability to motivate positive behaviours (Agrawal \& Duhachek, 2010; Boudewyns et al., 2013; Duhachek, Agrawal, \& Han, 2012). Although the effectiveness of fear appeals in message persuasiveness has been substantially explored, guilt and shame appeals remain relatively unexplored by comparison (Huhmann \& Brotherton, 1997; Witte \& Allen, 2000).

Health messages can challenge individuals' views of themselves as "adaptively and morally adequate" (Leary \& Baumeister, 2000, p. 262), leaving them with feelings attributed to guilt and shame. In this regard, therefore, guilt and shame can feasibly be used directly as appeals in health messages to motivate positive behaviour because of a threat posed to personal notions of self-integrity. However, there is little understanding of how and when guilt and shame appeals in health messages are most effective and, conversely, how and when these same messages may be counterproductive. Therefore, this proposed research will focus on the effectiveness of guilt and shame appeals on health communications by examining the difference between guilt and shame 
appeals, the coping response to the arousal of guilt and shame as a key mediator, and other key moderators.

Guilt is a negative emotion that occurs when a person believes his/her behaviour has violated personal or social standards (Izard, 1977). Guilt appeals have been shown to influence attitudes and intentions, but there is a debate regarding when they are most effective. Early studies have found that highly intense guilt appeals often cause anger and are less persuasive (referred to as an inverse-U relationship; Coulter \& Pinto, 1995), while moderately intense guilt appeals can elicit guilty feelings, thereby motivating people to change attitudes about a given product/offer (Coulter, Cotte, \& Moore, 1999; Coulter \& Pinto, 1995; Ghingold \& Bozinoff, 1982; O'Keefe, 2002; Pinto \& Priest, 1991; Pinto \& Worobetz, 1992). However, recent studies testing guilt appeals in both the prosocial and commercial realm attest to the effectiveness of intense guilt appeals (Cotte, Coulter, \& Moore, 2005; Lindsey, 2005; Turner \& Underhill, 2012) such that they did not support the inverse-U effect but rather supported a linear effect. This claim, further supported by Boudewyns et al. (2013), proposed that guilt is not correlated with anger, but rather that shame is associated with anger.

Shame differs from guilt, although people are often confused by these concepts (Boudewyns et al., 2013; Dearing, Stuewig, \& Tangney, 2005). Shame is a painful emotion aroused when the self is perceived to be defective, unworthy, and disgraceful (Lewis, 1992). Shame is associated with anger and even aggression, which has been empirically supported by several social psychology studies (Bennett, Sullivan, \& Lewis, 2005; Ferguson, Stegge, Miller, \& Olsen, 1999; Hoglund \& Nicholas, 1995; Stuewig, Tangney, Heigel, Harty, \& McCloskey, 2010; Tangney, 1999; Tangney \& Dearing, 2002). For instance, Tangney and Dearing (2002) found that while anger has been positively associated with shame-proneness, it has been negatively associated with guiltproneness. However, studies in communications have not only supported the claim but also raised the issue of confusion between guilt and shame appeals (Bennett, 1998; Boudewyns et al., 2013). Most importantly, Bennett (1998) suggested that messages designed to elicit highly intense levels of guilt may actually evoke shame feelings unintentionally. Similarly, Boudewyns et al. (2013) claimed that the highly intense guilt appeals that lead to anger actually evoked a combination of shame and guilt, or shame only. Some guilt appeals are actually shame appeals and this may provide a reason why 
intense guilt appeals are ineffective. Therefore, more research is needed to more fully understand the effects of guilt and shame separately.

In addition, guilt and shame are distinct and have differential effects in encouraging compliance (see Tangney, Stuewig, \& Mashek, 2007; Tracy \& Robins, 2007b). Guilt tends to create more favourable behavioural responses, but shame is more likely to trigger negative behaviours (Abe, 2004; Gausel \& Brown, 2012; Lindsay-Hartz, 1984). In social psychology, shame is a potent experience that plays an important role in normal development of both adaptive and maladaptive behaviours. Adaptively, shame essentially helps to motivate socially favourable behaviour and efforts towards self-improvement (de Hooge, Zeelenberg, \& Breugelmans, 2010; Gausel \& Leach, 2011; Lazarus, 1991a; Sznycer et al., 2016). However, when shame becomes a dominant emotion, it can lead to maladaptive outcomes (Gilbert, 1998; Kaufman, 1996; Lewis, 1995; Mills, 2005; Schore, 1998). In fact, shame has been found to have a strong relationship with many psychopathological symptoms. These include eating disorders, social anxiety, depression, and post-traumatic stress disorder symptoms (Averill, Diefenbach, Stanley, Breckenridge, \& Lusby, 2002; Gilbert, Allan, \& Goss, 1996; Tangney \& Dearing, 2002). Remarkably, the damaging aspects of shame are recognised consistently in psychology, but evidencebased interventions for health communications are noticeably absent (Boudewyns et al., 2013).

In terms of conceptualisation, the two emotions are definitely distinct (see Tangney et al., 2007, for review). For the outcomes, these constructs result in extensively different psychological and behavioural consequences (Tangney et al., 2007; Tracy \& Robins, 2007b). Researchers have claimed that the focus of blame attribution in shame is different from that associated with guilt, resulting in distinct phenomenological outcomes (e.g., Lewis, 1992). When people experience shame they tend to focus on themselves, not on their actions. But for guilt, the self is the subject not the object of the emotion, resulting in a focus on harm done to others or a relationship in relation to standards or rules (Tangney et al., 2007). Shamed people regret who they are and are more concerned with the past (e.g., hiding), while guilty people regret what they have done and are more concerned with the future (e.g., making amends) (Tangney et al., 2007).

However, guilt and shame both have been referred to as self-conscious emotions because they frequently involve perceptions of the self (e.g., Gao, Wang, \& Qian, 2010). These 
self-conscious emotions often carry extremely strong personal implications, therefore people have experienced these with health messages are highly motivated to make amends as a result of the personal significance in the message. In fact, they are particularly persuasive tools in health communications for reducing harmful behaviours, such as underage and binge drinking (Agrawal \& Duhachek, 2010). While a significant number of studies supporting development of guilt theory in the literature exists (Brennan \& Binney, 2010; Leshner, Bolls, \& Thomas, 2009), the theory of shame, especially in health communications, is underdeveloped.

The emotion of guilt or shame is an aversive experience, and it motivates an individual to try to ignore or avoid it (e.g., Czub, 2013). There are the two adaptive and maladaptive coping responses to these emotions (Lazarus, 1991a, 1991b; Schoenbachler \& Whittler, 1996). In the literature, given such a distinction has been overlooked by researchers; the specific variables through which emotions impact these distinct coping responses have not been investigated. Specifically, there has been no detailed discussion regarding how the evoking of guilt and shame affect the coping responses. It is argued that the intensity of emotional arousals elicited from guilt or shame appeals may have an impact on the coping responses (Agrawal \& Duhachek, 2010; Agrawal, Menon, \& Aaker, 2007; Dickinson \& Holmes, 2008). Indeed, Dickinson and Holmes (2008) found that higher emotional arousals from threat appeals did not lead adolescent individuals to respond adaptively. That is, while high physical threats produced the strongest emotional response, this does not, in turn, influence adaptive coping behaviour and message acceptance. Similarly, Agrawal and Duhachek (2010) found that a compatible message (e.g., a message that evokes the same emotion) is less effective in influencing behaviour and intentions due to it results in an emotional overload that drives people to shut down and avoid processing the message (Agrawal et al., 2007).

Given that guilt and shame appeals in threat-based messages raise ethical concerns due to explicitly using negative emotional feelings to try to manipulate human behaviour, there must first be reasonable justification for their use. First, there is a certain degree to which eliciting negative emotions is perceived to be fair. For example, anticipatory guilt could be construed as the least negative variation, since it centres on the future and the potential that individuals can avoid a negative emotion through their effort or exercise of selfcontrol (LaBarge \& Godek, 2006). This anticipatory guilt appeal has purely used the 
prospect of the negative emotion of guilt and implicitly prompted consumers to remember what experiencing guilt feels like. Second, shame appeals do not always have negative effects, and under the right circumstances can lead to positive outcomes (Wong \& Tsai, 2007). For example, in individualist cultures people may be harmed when shamed, but in collectivist cultures others may actually be helped. Finally, it has been argued that consumers can use their persuasion knowledge, known as what people know about marketers' persuasion efforts (Friestad \& Wright, 1994), to respond to advertisers' attempts. In fact, research confirms that consumers are active rather than passive processors of marketing stimuli by activating the role of persuasion knowledge as methods of coping with, and informing responses to negative emotional appeals (Coulter et al., 1999; Hibbert, Smith, Davies, \& Ireland, 2007; Lwin \& Phau, 2010).

Although a number of studies have examined the effectiveness of guilt and/or shame appeals, they have ignored some influential moderators. There is evidence that individual and cultural characteristics may influence message compliance in response to guilt and shame arousals. By examining these individual and cultural differences the study can identify conditions under which guilt and shame appeals are effective, in terms of message compliance. Message persuasion literature largely focused on message characteristics as moderators. However, the study investigates these moderators as individual characteristics. While the message characteristics are under the practitioners' control, it is possible to target specific audiences based on these individual differences (e.g., independent versus interdependent self-construals) through the choice of communication vehicles. For example, Facebook users who keep in contact with their family and close friends are likely to be interdependents (Martin, Lee, Weeks, \& Kaya, 2013).

Notably, an increasing body of literature suggests that the valuation, eliciting, and behavioural consequences of self-conscious emotions differ across cultures because of the type of self-construal that is promoted in one's cultural context (Lee \& Paek, 2014; Wong \& Tsai, 2007). That is, in contexts that promote an independent self (e.g., Western cultures), guilt is one of the valued emotions; however, in contexts that promote an interdependent view (e.g., Eastern cultures), shame is perceived more positively. Specifically, in a collectivist culture shame can lead to adaptive outcomes (Bagozzi, Verbeke, \& Gavino, 2003; Scollon, Koh, \& Au, 2011; Silfver, 2007; Wong \& Tsai, 
2007). For instance, Bagozzi et al. (2003) found shame improved customer relationshipbuilding as a result of adaptive resource use by Filipino salespersons, such as being flexible and adaptive in their interactions with clients. Thus, shame is self-regulated differently across cultures, and leads to differential effects on behavioural responses. Consequently, the role of culture, especially at individual level (e.g., personal cultural orientation, Yoo \& Donthu, 2005), is important in guilt and shame appeals, but this role is under-studied. Most studies of guilt and shame across cultures have been proposed and tested based mostly on national values, norms, and samples (Scollon et al., 2011). It is argued that in the case of coping responses to guilt and shame emotions, national culture may not apply. Thus, further advances in this area require exploring under which individual culture people up-regulate or down-regulate these two self-conscious emotions.

Regulatory focus has been conceptualised both as a malleable attribute that can be manipulated for a particular goal (e.g., message framing) and a stable individual difference variable (e.g., prevention-focused versus promotion-focused) (Higgins, 1997). Studies demonstrate that matching viewers' regulatory focus with the message's regulatory focus could be beneficial (Aaker \& Lee, 2001; Zhao \& Pechmann, 2007). That is, promotion-focused viewers are more persuaded by health messages that suggest behaviour changes lead to achievements (e.g., promotion-focused framing), whereas prevention-focused viewers are more persuaded by health messages suggesting that behaviour change results in threat reduction (e.g., prevention-focused framing). However, the role of viewers' regulatory focus in influencing the intensity of negative emotional arousals from guilt and shame appeals is not clearly understood.

Self-construal is defined as "a constellation of thoughts, feelings, and actions concerning the relationship of the self to others, and the self as distinct from others" (Singelis \& Sharkey, 1995, p. 624). While it is a more dynamic individual characteristic (Aaker \& Lee, 2001; Levinson, Langer, \& Rodebaugh, 2011), studies on self-construal are typically conducted through chronic cultural characteristics such as ethnicity. For instance, in Block's (2005) studies on the persuasiveness of fear and guilt appeals, the 'selfconstruals' were grouped into two kinds based on participants self-rating as American or Asian. Explaining the influence of self-construal as the dynamic individual characteristics of independence versus interdependence can be helpful to comprehend healthy, pro-social messages and compliance. 
Furthermore, guilt and shame appeals could be differently evaluated by referencing the transgression of non-adherence on the target (e.g., 'other-referenced') or those close to the target (e.g., 'self-referenced') (Burnkrant \& Unnava, 1995; Symons \& Johnson, 1997), and by the evaluation sources from the target's perspective (e.g., internal) or from others' perspectives (e.g., external) (Goss \& Allan, 2009; Grabhorn, Stenner, Stangier, \& Kaufhold, 2006; Lee, Scragg, \& Turner, 2001; Proeve \& Howells, 2002). The impact of guilt and shame appeals may be contingent on viewers' self-construal, and on the message's referencing or the sources of evaluation functioning synergistically. Little is known about the interactive effects between such message tactics and the individual characteristics of message receivers on guilt and shame appeals' effectiveness. This study, therefore, considers the interactive rather than main effects of such message characteristics and the individual characteristics of self-construal in health communications using guilt and shame appeals to better understand the effects of moderators on health message compliance.

\subsubsection{Research Gaps}

Given a growing literature has endeavoured to characterise the psychological processes involved in guilt and shame, often focusing on the distinct antecedents and their consequences for social behaviours, there is little understanding of how (e.g., mediators) and when (e.g., moderators) guilt and shame appeals in health communications can encourage the desired pro-social or pro-health behaviour. This is partly due to the lack of research into the distinction between guilt and shame effectiveness, the differing of emotional and psychological responses to the two types of emotions, and the key moderators that are influential in the response to them that motivate positive behaviours. Specifically, the current research focuses on examining the mediator of the coping response to emotional arousals, and the moderators of individual differences of self-construal (interacting with self-referencing, the sources of evaluation), regulatory focus, and personal cultural orientation influencing the relative effectiveness of guilt and shame appeals in health communications (see Figure 2.2).

These identified gaps are vital to fill as it is of great importance to get a better understanding of existing theory on the topic of guilt and shame appeals in health communications. First, it is important to examine the difference between guilt and shame appeals because health communications researchers and practitioners commonly use the 
terms 'guilt' and 'shame' interchangeably. A growing body of literature suggests that these constructs are distinct and generate widely divergent emotional and psychological responses (see Tangney et al., 2007; Tracy \& Robins, 2007b). Previously, such a distinction has been overlooked by researchers. Therefore, it is possible that guilt appeals have unintentionally elicited shame, which might account for some of the unintended effects that have been found in the literature (Bennett, 1998; Boudewyns et al., 2013). This study examines guilt and shame separately via distinct emotional guilt versus shame arousals rather than emotional appeals. By isolating the effect of an unintended emotion aroused from the appeal, the present study makes findings consistent and generalizable.

Second, it is important to study the mediating effect of the coping response to the arousal of guilt and shame to know the mechanism through which guilt and shame responses motivate compliance with health messages. Third, the three individual characteristics/moderators are important in order to explore conditions under which such appeals are effective. In other words, a significant contribution of the research is to identify boundary conditions on self-construal, regulatory focus, and personal cultural orientation guilt versus shame effects. Further, this study directly examine self-construal and personal cultural orientation - assumptions frequently made in the cross-cultural literature (Bagozzi et al., 2003; Kitayama, Mesquita, \& Karasawa, 2006; Yoo \& Donthu, 2005). Therefore, it has the potential to expand our knowledge of how guilt and shame function across cultures.

By focusing attention on both effects - mediating and moderating - this work provides full insights into the different emotional and psychological responses that result from guilt and shame emotions. It provides additional clarity to the guilt and shame literature. And finally, it provides a new approach for examining cross-cultural differences in selfconscious emotions.

\subsection{Research Questions and Objectives}

Against the aforementioned background, the current research contributes to literature in the domain of health communications and emotions by answering the following research questions: 
1. Do self-construal, regulatory focus and personal cultural orientation influence the relative effectiveness of guilt and shame appeals towards health message compliance?

2. Does the coping response to guilt and shame arousals influence health message compliance?

To address the above research questions, further objectives are as follows:

1. To investigate the impact of self-construal, regulatory focus and personal cultural orientation on the effectiveness of guilt and shame appeals in health communications.

2. To investigate the impact of coping responses to guilt and shame arousals on message compliance.

3. To examine differences in the effectiveness of guilt versus shame appeals towards health message compliance.

4. To examine the mechanism through which guilt and shame appeals motivate health message compliance. Specifically, to examine the mediating roles of guilt and shame arousals and coping responses, and the moderating roles of selfconstrual (interacting with self-referencing and the sources of evaluation), regulatory focus, and personal cultural orientation.

\subsection{Research Methodology}

This proposed empirical study builds and tests a theoretical framework for the processes by which guilt and shame appeals may lead to better compliance with health messages. This research involves multiple psychological mechanisms and multiple moderators.

This study investigates the research questions in the context of health communications, binge drinking in particular, with a sample of undergraduate students in New Zealand. A series of 2 emotion (guilt versus shame) x 2 self-referencing (self-reference versus otherreference) x 2 source of evaluation (internal versus external) between-subjects experiments was conducted in an online survey to test the research model. A set of stimulus materials using print advertisements (ads) was developed. The ads in the study differentiated guilt and shame, and were based on the results of pretests.

Based on the survey data, this study applied structural equation modelling and regression analyses to test the hypotheses and address the research questions. Specifically, structural 
equation models were estimated using AMOS to test hypotheses regarding the main structural relationships. In addition, multiple sample analyses were conducted to test the moderation effects of self-construal, regulatory focus, and personal cultural orientation. The structural equation model of latent interactions was estimated to moderating effect of tie strength. A two-way ANCOVA using SPSS was conducted to test the hypotheses associated with the interaction effects of self-construal with self-referencing or the sources of evaluation.

\subsection{Intended Contributions}

The study offers several theoretical and practical contributions to the literature on health communications and discrete emotions.

\subsubsection{Theoretical Contributions}

Regarding theoretical contributions, the current research attempts to build a model of the effectiveness of guilt versus shame appeals on health message compliance. This research has several important aspects. Firstly, it considers the effects of coping responses to emotions, a mediating factor which is under-studied but underlies health message compliance to better understand the theoretical premise of self-conscious emotional appeal messages. Secondly, it examines the interactive rather than main effects of moderating factors, i.e., self-referencing and the sources of evaluation with self-construal. These interactive effects help better predict the effects of individual differences in selfconstrual on self-conscious emotional appeal messages. Thirdly, this research considers the effects of additional moderating factors, i.e., viewers' regulatory focus and personal cultural orientation, which have also been under-researched in the field of guilt and shame emotions. This aspect of the research identifies boundary conditions on which guilt versus shame effects are brought about by regulatory focus and personal cultural orientation. Fourthly, this research examines the differential effects of guilt versus shame appeals in the health communications context. This provides understanding of the importance of personal significance in this context. The much-researched pro-social and donation behaviour context does not include personal significance. Taken together, these focal aspects better predict the effectiveness of guilt versus shame appeals on health message compliance. 


\subsubsection{Managerial Implications}

Regarding practical contributions, guilt and shame as self-conscious emotions frequently involve perceptions of the self, and are thus particularly persuasive tools for health communicators in addressing a wide range of unhealthy behaviours, such as obesity, underage and binge drinking, and drinking and driving. Besides highlighting guilt and shame as they relate to harmful behaviours (e.g., unhealthy eating, not exercising), health communicators could focus on the idea of self-affirmation (i.e., the affirmation of values that are important to the self, see de Wit, Das, \& de Hoog, 2007) to trigger adaptive responses that make receivers more accepting of health messages. In addition, health messages can centre on the role of the evaluation sources or transgression of a person with respect to others (e.g., self-referenced versus other-referenced, internal versus external source of evaluation). The use of such message tactics definitely enhances the effectiveness of guilt and shame appeals in practice. Also, while the message characteristics (e.g., self-referencing, source of evaluation) are under the marketers' control, it is possible to target specific audiences (e.g., self-construal, regulatory focus, personal cultural orientation) through the choice of media programmes/channels as communication vehicles. This study, therefore, offers significant practical benefits for health marketers, health promotion agencies, and policy makers.

\subsection{Outline of the Thesis}

The thesis comprises seven chapters. This chapter briefly discusses the research background, research gaps, research objectives and questions, research methodology and expected research contributions. The remainder of this thesis is structured as follows: Chapter 2 reviews the relevant literature that provides theoretical foundations for this research: guilt versus shame and theoretical underpinnings. The development of the key constructs, hypotheses, and the conceptual framework are discussed in Chapter 3. Chapter 4 describes the methodology of this study, including the operational measures of the constructs, the experiment development and stimulus pretest, the data collection procedures and data analysis strategies. Chapter 5 presents the results of the empirical analysis and hypotheses testing. Chapter 6 comprises an in-depth discussion of the test results while reflecting back on the literature. In Chapter 7, this research concludes with a discussion of the theoretical and methodological contributions, and practical implications. 
This chapter also discusses the study's limitations with suggestions for future research. References and appendices follow Chapter 7.

\subsection{Chapter Summary}

The chapter has introduced this work by a brief discussion of the motivation for the research, research gaps, objectives, questions and methodology description, and the theoretical and practical contributions. An overview of the conceptual model to be developed has been outlined drawing on the theoretical frameworks of cognition, emotion and motivation, and empirical studies focusing on guilt and shame appeals and effectiveness. The conceptual model incorporates both influential mediating and moderating variables. These include the mediator (e.g., coping response to emotion) and the moderators (e.g., self-construal, regulatory focus, and personal cultural orientation). The next chapter, the literature review, now follows. 


\section{Chapter 2. Literature Review}

\subsection{Introduction}

This chapter reviews the major literature on guilt, shame and pro-social and/or health communications that is pertinent to the study. First, it discusses the definition and distinction of guilt and shame, and the theoretical underpinnings. Next, a review of studies on the subject from the pro-social and charitable donation behaviour literature that examines studies of guilt and/or shame appeals' effectiveness is presented. The next section proposes the model of guilt and shame appeals in health communications based on the literature reviewed, examining the mediating and moderating variables influencing the effects of guilt and shame appeals on health communications.

\subsection{Guilt and Shame: Definition and Characteristics}

Guilt and shame are perceived as intense, negative self-conscious emotions (Gao et al., 2010). They are characteristically more self-evaluative than basic emotions and, as such, are dependent on sophisticated cognitive processes (Kim, Thibodeau, \& Jorgensen, 2011). The cognitive prerequisites for guilt and shame include: (i) self-awareness and a stable sense of 'self-as-separate-from-other' (Lewis \& Brooks-Gunn, 1979); (ii) the ability to direct attention towards the self and to self-reflect; (iii) an understanding that the self is the agent of behaviour (Kagan, 1981); (iv) the apprehension, endorsement, and internalisation of standards for behaviour (Stipek, Recchia, \& McClintic, 1992); (v) an ability to identify discrepancies between these standards and the self or its behaviour; and (vi) attributions, e.g., the processes of explaining the causes of events (Weiner, 1985). Conversely, this array of developmentally advanced cognitive skills is seen as unnecessary for basic emotions.

While basic emotions, such as fear or anger, are assumed to have primarily evolved to address immediate and typically urgent physical survival-related threats, opportunities and reproduction, self-conscious emotions such as guilt and shame have evolved to address social survival-related problems and opportunities (Levenson, 1999; Plutchik, 1980; Tooby \& Cosmides, 1990). Basic emotions should be experienced similarly across cultures subject to the same universal concerns (Ekman \& Friesen, 1975; Ellsworth, 1994). But, there is substantial cross-cultural divergence with respect to several elements of self-conscious emotions (Wong \& Tsai, 2007), including the value, subjective 
experience, and antecedent events that give rise to such emotions. This is because there are cross-cultural differences in standards and attributions. For example, according to Kitayama and Park (2007), different cultures have differing views of self, which are influential in defining daily routines, practices, and public meanings. As a result, culture has a strong impact on emotion, wellbeing, and health (Mesquita, 2001; Tracy \& Robins, 2007a).

\subsubsection{Guilt Appeals}

Guilt is defined as "an aversive conscious emotion that involves criticism of, and remorse for, one's thoughts, feelings, or actions" (Blum, 2008, p. 97). A typical guilt-evoking circumstance is one in which a person has acted in some manner inconsistent with his/her own conception of proper conduct (O'Keefe, 2002). For example, the sorts of situations that people recall as especially associated with guilt are ones that involve conduct such as lying, cheating, stealing, neglecting others, or failing to perform duties (Keltner \& Buswell, 1996). This suggests that guilt involves a particular behaviour that one selfperceives as a shortfall compared to his/her own standards. Notably, guilt is accompanied by feelings of remorse, accountability and responsibility that motivate people to take action to repair the committed transgression in order to reduce negative feelings (Izard, 1977). Therefore, guilt has been considered an emotion that can be constructed in positive ways to motivate individuals to follow a recommended action (Huhmann \& Brotherton, 1997). Accordingly, guilt appeals are persuasive messages designed to call for remedial action (O'Keefe, 2000). Guilt appeals have a 'problem-solution' structure: when the individual's conduct violates their personal, social, and moral norms and principles, they experience guilt and take the recommended course of action to reduce those feelings. In other words, by directly or indirectly challenging people with inconsistencies between their internalised standards and actual behaviour, guilt appeals can create an aversive feeling that will encourage them to seek relief by making amends for their self-perceived shortfalls. For an example of a guilt appeal, see Table 2.3.

There are three types of guilt recognised in the literature: reactive, anticipatory, and existential guilt (see Huhmann \& Brotherton, 1997), which differ in their antecedents. Reactive guilt is a response to having violated one's standards of acceptable behaviour (Rawlings, 1970). For example a viewer who, having watched an ad, realises they previously neglected their children's dental care (violating their own personal standards) 
might purchase dental floss suggested by the ad to alleviate the evoked feelings of guilt. Anticipatory guilt occurs when one anticipates a potential violation of one's internalised standards (Rawlings, 1970). Anticipatory guilt appeals provide an opportunity for receivers to avoid a transgression, but an unwanted outcome will occur if the opportunity is neglected. For instance, life insurance ads have tried to elicit guilt in parents by portraying unwanted outcomes that they could have prevented by buying a suggested policy for their children. Existential guilt is experienced as a result of the awareness of a discrepancy between one's well-being and the well-being of others (Izard, 1977). Such a discrepancy occurs when one feels better off, or more fortunate than others, resulting in feelings of empathy. For that reason, charity messages often use existential guilt appeals to trigger the audience's feelings of responsibility for easing the suffering from poverty, famine, or natural disasters (Lill, Gross, \& Peterson, 1986).

The effects of guilt appeals on attitude change and behavioural intentions have found significant support in the literature (Alden \& Crowley, 1995; Bécheur, Dib, Merunka, \& Valette-Florence, 2007; Bennett, 1998; Cotte et al., 2005; Ghingold, 1980; Lindsey, 2005; Pinto \& Priest, 1991). For instance, studies have demonstrated that guilt can impact consumers' decision-making process (Burnett \& Lunsford, 1994), attitude towards the ad (Cotte et al., 2005), and attitude towards the brand (Coulter \& Pinto, 1995; LaBarge \& Godek, 2006); and it can motivate charitable behaviours (Hibbert et al., 2007) and reduce risky behaviours like substance use (Dearing, Stuewig, \& Tangney, 2005). However, the use of guilt appeals is controversial regarding when they are most effective. Early studies found that highly intense guilt appeals are less persuasive (Coulter \& Pinto, 1995; Pinto \& Priest, 1991; Pinto \& Worobetz, 1992). For example, a study in consumer marketing by Coulter and Pinto (1995) showed that moderate and intense guilt appeals evoke stronger feelings of guilt than low intensity ones, but such guilt appeals generate anger and a perception of manipulative intent, and consequently purchase intention declines. This is because strong guilt appeals are viewed as attacks on the self and efforts to limit one's freedom by criticising one's conduct (Burgoon, Alvaro, Grandpre, \& Voulodakis, 2002). Likewise, others found intense guilt appeals result in psychological reactance, such as resentment, anger and a desire to lash out against the message and the messenger (Stark \& Frenkel, 2013). This is similar with the inverse-U effect in fear appeals (see Bennett, 1996), but support for this interpretation is somewhat conflicted. For example, recent studies testing guilt appeals in the pro-social and commercial realm attest to the 
effectiveness of intense guilt appeals such that they did not support the inverse-U effect, but rather supported a linear effect (Cotte et al., 2005; Lindsey, 2005; Turner \& Underhill, 2012).

Bennett (1998) identified that while the intense guilt appeals are designed to evoke guilt, they could elicit shame feelings unintentionally. This may be an explanation for why intense guilt appeals [not generating anger] are ineffective because they are actually evoking shame [generating anger] (Boudewyns et al., 2013). In the literature, there are studies using stimuli of intense guilt appeals that actually evoke shame. For instance, in Coulter and Pinto's (1995) consumer advertising studies, their intense guilt appeal for dental floss stated: "It's YOUR responsibility to make sure that your kids have healthy teeth and gums. The pressure is on YOU, so don't make any mistakes... DO IT RIGHT!" (p. 700). Clearly, the stimulus seems to emphasise the word 'you' that implies the person instead of behaviour; as a result, intense guilt appeals result in shame rather than guilt.

\subsubsection{Shame Appeals}

Shame is a painful self-conscious emotion brought about by an evaluation of failure in relation to internalised standards when an evaluation of the global self is made (Lewis, 1992). Shame often induces additional feelings such as embarrassment, shortfall, and selfcontempt (Tangney \& Dearing, 2002). Most define shame as a negative experience. For instance, Kaufman (1996) states that shame is the source of feelings of inferiority, and the inner experience of shame is like a sickness of the soul. Stuewig and McCloskey (2005) refer to shame as a negative emotion focusing on evaluation of the global self with internalised standards. Similarly, Lewis (1995) and Tangney (1995) describe shame as a negative experience involving defensive reactions such as self-condemnation and the desire to hide from others. Lewis (2000) concludes that shame is a self-conscious emotion requiring cognition of self and the ability to evaluate the global self against a standard and recognise one's failure. Miller (2012) describes the experience of shame as an inner, critical voice that judges a person's actions as wrong, inferior, or worthless. Notably, shame is a forceful experience that plays an important role in normal development for both adaptive and maladaptive manners. Adaptively, shame is essentially helping to motivate socially favourable behaviour and efforts to improve the self (Lazarus, 1991a; Sznycer et al., 2016). However, when shame becomes a dominant emotion, it can lead to 
maladaptive outcomes (Gilbert, 1998; Kaufman, 1996; Lewis, 1995; Mills, 2005; Schore, 1998).

Shame is associated with defensive reactions such as anger, resentment, and lashing out (Lewis, 1971; Tangney, 1993; Tangney \& Dearing, 2002). Anger may be evoked through an emphasis on others' sense of self in the shame appeals, such as through the use of the word 'you' (Niederdeppe, Bu, Borah, Kindig, \& Robert, 2008). The reason for this reaction is that the use of the second-person pronoun implies persuasive intent, which has been shown to be counter-productive. This supports a claim that perceptions of manipulative intent do not necessarily block guilt feelings; instead, they result in shame feelings (Boudewyns et al., 2013).

According to Bagozzi et al. (2003), the experience of shame has four dimensions: (i) selffocused attention (e.g., the self as an object for evaluation); (ii) the core self is threatened (a failure to meet the expectations imposed by others such as friends, family members); (iii) awareness of additional physiological symptoms (a bit edgy, fidgety, shaky, physically weak, dizzy feelings); and (iv) having the urge to hide or run away. Thus shame appeals can be defined as persuasive messages designed to urge attention to a (or anticipated) failure and call for an respected behaviour, and it focuses on the self rather than the behaviour (Boudewyns et al., 2013). For an example of a shame appeal, see Table 2.2.

There are many functional interpretations for shame established in the literature (Sznycer et al., 2016). Shame has been known to mobilise withdrawal, which protects the transgressor against acts immediately motivated by devaluation (Tangney, Miller, Flicker, \& Barlow, 1996; Wicker, Payne, \& Morgan, 1983) and weakens the formation of common knowledge of the shameful act (Thomas, DeScioli, Haque, \& Pinker, 2014). Each submission (Gilbert, 2000), appeasement (Keltner, Young, \& Buswell, 1997), and cooperation (de Hooge, Breugelmans, \& Zeelenberg, 2008) would function to increase the value of the transgressor after devaluation. Sometimes, aggression occurs when threatening harm is an effective way of preventing the spread of negative information or when it is the best way to bargain for better treatment (Fessler, 2001; Tangney, Wagner, Fletcher, \& Gramzow, 1992). 


\subsubsection{Distinction between Guilt and Shame Appeals}

Despite often being used interchangeably, guilt and shame have important conceptual differences (Cleary, 1992; Stuewig \& McCloskey, 2005; Teroni \& Deonna, 2008). The major difference involves the differing foci of attention between the self and behaviour (Stuewig \& McCloskey, 2005). In shame, the focus is on the global self, whereas in guilt it is on a specific behaviour (Feiring \& Taska, 2005; Lewis, 1971; Tangney, 1992, 1995). A person might be ashamed of who s/he is, but feel guilty about what s/he did (O'Keefe, 2002).

Guilt and shame are separate emotions (see Tangney \& Dearing, 2002). Firstly, as discussed, guilt is a negative feeling that is experienced when individuals appraise negative outcomes to their specific behaviour (Blum, 2008; Lewis, 1971). Guilt-laden individuals are likely to blame a specific behaviour for the negative outcomes rather than view their global self negatively (Blum, 2008; Tangney \& Dearing, 2002; Tangney, Stuewig, \& Mashek, 2007). For instance, research shows that individuals who attribute poor performance on their specific errors, e.g., "I did not study hard", are more likely to experience feelings of guilt (Tangney et al., 1992). In contrast, shame is a negative feeling experienced when individuals blame negative events to their entire shortfalls (Lewis, 1971; Lewis, 2000; Van Vliet, 2009). Individuals who experience feelings of shame tend to centre on the deficiency of their global selves (Lewis, 1992, 2000; Tangney, 1995; Tangney \& Dearing, 2002) and blame themselves as a whole for negative outcomes (Van Vliet, 2009). Research also shows that individuals who attribute poor performance on their global deficiency, e.g., "I'm an unintelligent person", are more likely to feel shame (Tangney et al., 1992).

A second distinction between guilt and shame aligns guilt with subsequent approach behaviours and shame with avoidance behaviours (see Tangney et al., 2007). Specifically, guilt increases empathy and the desire to apologise and make amends, whereas shame prompts the desire to escape from social attention and one's own awareness of the transgression (Lindsay-Hartz, 1984; Tangney, Wagner, Hill-Barlow, Marschall, \& Gramzow, 1996). Shame has also been linked to cognitive-attributional reactions like externalising blame for the transgression, which appears to foster anger and aggression (Dean \& Fles, 2016; Stuewig et al., 2010). 
In addition, shame feelings result in feelings that are painful and disabling for individuals, putting one's core sense of self in threat (Lewis, 1971; Lindsay-Hartz, de Rivera, \& Mascolo, 1995). Shame may cause a self-defeating cycle of negative affect and substance abuse due to struggling to ease this painful feeling with drugs or alcohol. In contrast, guilt is painful but less debilitating, and is likely to encourage people to seek relief by making reparation or behavioural change (Baumeister, Stillwell, \& Heatherton, 1995).

Table 2.1 below shows the critical differences between guilt and shame. For a review of distinct self-evaluative antecedents and consequences for social behaviour, see Tangney et al. (2007).

Table 2.1. Differences between Shame and Guilt

\begin{tabular}{|c|c|c|c|}
\hline Dimension & Guilt & Shame & Sources \\
\hline \multicolumn{4}{|l|}{ Conceptualisation } \\
\hline $\begin{array}{l}\text { Object of negative } \\
\text { evaluation }\end{array}$ & $\begin{array}{l}\text { A specific behaviour/ } \\
\text { action }\end{array}$ & The global self & $\begin{array}{l}\text { Lewis (1971); } \\
\text { Tangney and } \\
\text { Dearing (2002) }\end{array}$ \\
\hline Formal object & The superego & The ego-ideal & $\begin{array}{l}\text { Lynd (1958); Piers } \\
\text { and Singer (1971) }\end{array}$ \\
\hline Context & Internal sanction & Social sanction & $\begin{array}{l}\text { Teroni and Deonna } \\
(2008)\end{array}$ \\
\hline Phenomenology & $\begin{array}{l}\text { Tension, regret, } \\
\text { remorse, other-oriented } \\
\text { concern }\end{array}$ & $\begin{array}{l}\text { Feeling 'small' and inferior, } \\
\text { helpless, powerless, } \\
\text { exposed }\end{array}$ & $\begin{array}{l}\text { Lindsay-Hartz } \\
\text { (1984); Tangney and } \\
\text { Dearing (2002) }\end{array}$ \\
\hline \multicolumn{4}{|l|}{ Outcomes } \\
\hline Action tendencies & $\begin{array}{l}\text { Approach, amendment, } \\
\text { reparation, confession, } \\
\text { apology }\end{array}$ & $\begin{array}{l}\text { Avoidance, hiding, } \\
\text { withdrawal, escapism, self- } \\
\text { isolation, a desire to } \\
\text { 'disappear' }\end{array}$ & $\begin{array}{l}\text { Frijda, Kuipers, and } \\
\text { Ter Schure (1989) }\end{array}$ \\
\hline $\begin{array}{l}\text { Accompanying } \\
\text { emotions }\end{array}$ & $\begin{array}{l}\text { Sorrow and empathy; } \\
\text { typically less painful }\end{array}$ & $\begin{array}{l}\text { Anger, anxiety, (self-) } \\
\text { disgust; typically more } \\
\text { painful }\end{array}$ & Gilbert (1998) \\
\hline
\end{tabular}

The distinct behavioural consequence that shame is more likely leads to avoidance behaviours while guilt more likely leads to subsequent approach behaviours has been long established in past research (Tangney et al., 2007; Tracy \& Robins, 2007b). However, recent studies of shame critique this rationale that shame is associated with greater desire to change aspects of one's self implicated in a past transgression, activates approach action tendencies (Gausel \& Brown, 2012; Sznycer et al., 2016). Specifically; for shame 
the desire to protect one's self-image prompts self-enhancement and the desire to restore the integrity of the self prompts behaviour aimed at self-improvement (e.g., Dean \& Fles, 2016). Therefore, shame and guilt stems from negative evaluations of the self versus behaviour, but elicits positive behavioural consequences as a function of self-conscious emotions.

Guilt and shame both have been referred to as self-conscious emotions because they frequently involve perceptions of the self as previously mentioned in Section 2.2 on their characteristics (Gao, Wang, \& Qian, 2010). The self-conscious emotions of guilt and shame often carry extremely strong personal implications, therefore people have experienced these with health messages are highly motivated to make amends as a result of the personal significance in the message. That is, guilt and shame feelings motivate pro-health behaviour and efforts towards self-improvement because of a threat posed to personal notions of self-integrity (Leary \& Baumeister, 2000; Sznycer et al., 2016). In fact, both guilt and shame appeals are particularly persuasive tools in health communications for reducing harmful behaviours, such as binge and underage drinking (Agrawal \& Duhachek, 2010).

Table 2.2 below shows the crucial similarities between guilt and shame in terms of conceptualisation and outcomes.

Table 2.2. Similarities between Shame and Guilt

\begin{tabular}{|c|c|c|}
\hline Dimension & Both Guilt and Shame & Sources \\
\hline \multicolumn{3}{|l|}{ Conceptualisation } \\
\hline $\begin{array}{l}\text { Characteristics of } \\
\text { perception }\end{array}$ & $\begin{array}{l}\text { Self-conscious emotions: self-awareness, self- } \\
\text { evaluation }\end{array}$ & $\begin{array}{l}\text { Gao et al. (2010); } \\
\text { Kim et al. (2011); } \\
\text { Tracy and Robins } \\
\text { (2007a); Tracy ano } \\
\text { Robins (2007b) }\end{array}$ \\
\hline \multicolumn{3}{|l|}{ Outcomes } \\
\hline Action tendencies & $\begin{array}{l}\text { Motivate people to make amends: resultant pro-health } \\
\text { behaviour and efforts towards self-improvement } \\
\text { because of the personal significance and personal } \\
\text { notions of self-integrity posed by a threat }\end{array}$ & $\begin{array}{l}\text { Agrawal and } \\
\text { Duhachek (2010); } \\
\text { Dean and Fles } \\
\text { (2016); Sznycer et } \\
\text { al. (2016) }\end{array}$ \\
\hline
\end{tabular}

For engineering a guilt-inducing or shame-reducing appeal, Tangney and Dearing (2002) have created some practical guidelines, including emphasising the behaviour, not the self, 
involving the consequences for others, and guiding the development of reparative skills. It should also be noted that there is the tactical option of varying the degree of shame appeals. According to Boudewyns et al. (2013), rather than choosing the common tactic of using more severe language or more emotionally overloaded pictures, a better way to thoroughly intensify shame is to vary the intensity of empathy and efficacy comprised in the appeal. In this approach, a high-shame appeal would de-emphasise the consequences for others, cut-off the efficacy cue, and focus on the self. These guidelines are applied to the manipulation of guilt versus shame appeals for the current research (see Table 4.2). Table 2.3 summarises health examples of guilt and shame appeals in the literature.

Table 2.3. Health Examples of Guilt and Shame Appeals

\begin{tabular}{|c|c|}
\hline Guilt appeal & Shame appeal \\
\hline WHAT WOULD GIVE YOUR PARTNER AN & WHO WOULD GIVE THEIR PARTNER AN \\
\hline STD? & STD? \\
\hline A. Someone with forgetful behaviour. & A. An immature person. \\
\hline B. Someone with uninformed behaviour. & B. A selfish person. \\
\hline C. Someone with unreliable behaviour. & C. An irresponsible person. \\
\hline D. Someone who hasn't been tested for STDs. & D. An untested person. \\
\hline E. All of the above. & E. All of the above. \\
\hline
\end{tabular}

By the age of 25 , one in two young people will give a sexually transmitted disease (STD) to someone else. Most won't know it because many STDs have no symptoms. If you haven't been tested, that doesn't mean you are a bad person, but STDs have serious consequences for both you and your sexual partner. So if you have ever had sex without a condom you should be tested for STDs.

SHOW YOUR PARTNER RESPECT. GET TESTED FOR STDS.

Source: Boudewyns et al. (2013, pp. 824-825)

In sum, the two emotions of guilt and shame differ in their attributional conceptualisations. However, there is disagreement regarding their distinct behavioural consequences. Continuing in this vein, the current study examines the roles of mediator of coping response and moderators including self-construal interacting with self-referencing or sources of evaluation, regulatory-focus and personal cultural orientation in order to distinguish guilt and shame, specifically their appeals' effects in message compliance. 


\subsection{Theoretical Underpinnings}

The objective of this present research is to develop an extended model of the effectiveness of guilt and shame appeals on health communications. Specifically, the study examines the roles of the coping response to emotional arousals and individual differences of self-construal, regulatory focus, and personal cultural orientation in the relative effectiveness of guilt and shame appeals. In order to provide foundations to the current research, theoretical insights are reviewed. The review commences with the Cognitive-Motivational-Relational Theory of Emotion. The theory provides the primary foundation for this study's proposed model as it: $(i)$ specifies how the different emotions are aroused and how each influences subsequent actions and reactions, while this study examines the differential between guilt and shame; (ii) differentiates between coping and action tendencies, specifically coping follows emotion and shapes negative emotions, while this study examines coping response as a key construct. This is followed by Reactance Theory offering the foundations for some emotional reactions to negative emotional appeals. There are two types of emotional reactions experienced in response to guilt and shame appeals: $(i)$ the negative emotion feelings intended by the messenger to stimulate action, such as guilt and shame feelings; ( $i i)$ the subsequent negative emotions or defensive reactions, such as anger, that are likely not intended by the messenger (and arise due to the individuals activating their persuasion knowledge). Next is the Persuasion Knowledge Model describing how message viewers use their persuasion knowledge to respond to influence attempts from messengers. It is used to explain why perceptions of manipulative intent brought about by intense guilt/shame appeals result in psychological reactance, such as anger as previously mentioned. Lastly, Regulatory Focus Theory constructing regulatory focus as an influential moderator in this study is drawn on.

\subsubsection{Cognitive-Motivational-Relational Theory of Emotion}

The Cognitive-Motivational-Relational Theory of Emotion (Lazarus, 1991a, 1991b) provides the primary foundation for this study's proposed model. It specifies how the different emotions are aroused and how each powers subsequent actions and reactions. The theory is relational, motivational, and cognitive. Relationally, emotions are always about person-environment relationships that encompass benefits from positive emotions and harm from negative emotions. Motivationally, acute moods/emotions are responses to 
the status of everyday, or our lives' overall, goals/adaptational encounters. The term cognitive relates to the knowledge and appraisal of what is happening in the adaptational encounters of living. Knowledge consists of situational and generalised beliefs about how things work, while appraisal consists of an evaluation of the personal significance of what is happening in an encounter with the environment.

Significantly, the theory differentiates between coping and action tendencies (Lazarus, 2000). Whereas coping is the psychological signal of action tendencies, action tendencies appear to be biologically given and therefore relatively rigid and automatic. From this perspective, coping is a key variable in the emotions, especially the negative ones elicited from harm and threat. Accordingly, coping follows emotion, e.g., emotion-focused coping, which is designed to self-regulate emotional suffering, and is the traditional way in which coping is conceptualised. Moreover, coping shapes subsequent feelings, a direction of effect that has been under-emphasised in traditional coping theory. For example, coping shapes negative emotions in one of two ways: $(i)$ problem-focused coping often involves planned actions to change the actual person-environment relationship by directly acting on the environment or on oneself, and this is referred to as adaptive coping; and (ii) emotion-focused coping alters only what is in the mind in one of two ways, either by attention deployment (e.g., avoidance) or by changing the meaning of the relationship (e.g., denial or distancing), and this is referred to as maladaptive coping.

\subsubsection{Reactance Theory}

Reactance Theory (Brehm, 1966) offers the foundations for some emotional reactions to negative emotion appeals. That is, if people believe that a message appeal (e.g., guilt or shame) is trying to force a response, they feel threatened and will react negatively to their perceived loss of freedom. Support for this defensive reaction can be found in the literature. For example, studies have found that people viewing a guilt ad reported higher levels of anger, disdain, and disgust (Coulter \& Pinto, 1995; Englis, 1990). Similarly, another study found that as the intensity of the guilt appeal increased, participants reported increased anger and fewer positive emotional reactions (Pinto \& Priest, 1991). As a result, there are two types of emotional reactions experienced in response to guilt and shame appeals. The first type is the negative emotion feelings intended by the messenger to stimulate action, such as guilt and shame feelings. The second type is the subsequent negative emotions or defensive reactions, such as anger, resentment, 
annoyance and lashing out that are likely not intended by the messenger, and arise due to the individuals activating their persuasion knowledge (Cotte et al., 2005; Coulter, Cotte, $\&$ Moore, 1997). Coulter and Pinto (1995) found that these unintended emotions affect attitudes towards the message and the messenger to such a great extent that they overwhelm the feelings of guilt and shame in forming attitude and behaviour intentions.

\subsubsection{Persuasion Knowledge Model}

The Persuasion Knowledge Model (Friestad \& Wright, 1994) describes how people use their persuasion knowledge to interpret, evaluate, and respond to influence attempts from marketers. The model presumes that people's persuasion knowledge is developmentally contingent (Campbell \& Kirmani, 2000). Within individuals, it continues developing over time and it is also, to some extent, historically contingent. People can learn about persuasion in numerous ways: from straight experiences involving family, friends, and colleagues; from discussions about what influenced people's thoughts, attitudes, and behaviours; from marketers' and other persuasion agents' observation; and from the mass media's commentary on marketing persuasive tactics, and so on. As a result, over time, the impact of these actions by known persuasion agents (e.g., advertisers, promoters, salespeople) on consumers' attitudes and behaviour will change, because the persuasion knowledge obtained will shape responses by consumers as persuasion targets.

In the literature, research has found that intense guilt appeals generate perceptions of manipulative intent as a result of activating receivers' persuasion knowledge, and consequently behavioural intentions ultimately decline. That is because strong guilt appeals are viewed by consumers as attacks on their self and as efforts to limit their freedom by criticising their conduct (Burgoon et al., 2002). Perceptions of manipulative intent brought about by intense guilt appeals result in psychological reactance, such as resentment, anger and a desire to lash out against the message and the messenger (Stark \& Frenkel, 2013). Noted that, regarding anger there is confusion between guilt and shame appeals existing in the literature (Bennett, 1998; Boudewyns et al., 2013). That is, messages designed to elicit highly intense levels of guilt may actually evoke shame feelings unintentionally. Thus, the highly intense guilt appeals that lead to anger actually evoked a combination of shame and guilt, or shame only.

It is argued that the consumers' persuasion knowledge varies across cultures due to peer conformity, and marketplace knowledge (e.g., advertising exposure). Peer group 
conformity was shown to be negatively related to ad scepticism (Mangleburg \& Bristol, 1998). People from Eastern cultures are often more concerned with peer conformity (being from collectivist societies), are relatively less sceptical of advertising and are less likely to perceive manipulative intent (Schaefer, Hermans, \& Parker, 2005; Sinh, 2013, 2014). Market knowledge was shown to be positively related to advertising exposure (Mangleburg \& Bristol, 1998; Schaefer et al., 2005). Heavy exposure to advertising has fostered in people a familiarity with advertising tactics and provided opportunities to test ad truthfulness through their personal purchase experiences. Thus people with greater marketing exposure (e.g., Americans) will have a greater tendency towards scepticism and perceptions of manipulative intent.

\subsubsection{Regulatory Focus Theory}

Regulatory Focus Theory (Higgins, 1997, 1998) is proposed as the foundation for an individual variable in this study. It posits that there are two distinct types of regulatory systems concerned with meeting the basic needs for nurturance and gain (e.g., promotionfocused), and for security and safety (e.g., prevention-focused). The two self-regulatory systems involve an approach motivation such that self-regulation towards any specific goal may be focused on promotion, e.g., the pursuit of gains and aspiration towards ideals or, alternatively, may be focused on prevention, e.g., the avoidance of losses and the fulfilment of obligations. These distinct motivational patterns of promotion versus prevention focus have been shown to powerfully predict cognition and affect (Aaker \& Lee, 2001).

Regulatory focus has been conceptualised both as: (i) a malleable attribute that can be manipulated for a particular goal (e.g., message framing and presentation), and (ii) a stable individual difference variable (e.g., prevention-focused versus promotion-focused). In terms of the latter, individuals with a promotion focus direct their behaviour towards events they expect to have a positive outcome, while individuals with a prevention focus will act in such a way as to avoid events they expect to have a negative outcome (Aaker $\&$ Lee, 2001). It could thus be expected that individuals with a prevention focus will experience a more negative emotional response to both guilt and shame appeals as compared to individuals with a promotion focus. This is because the feelings evoked by guilt and shame appeals are all painful emotions. The congruence between regulatory focus and message presentation is supported by research in the area of message framing. 
For instance, Lee and Aaker (2002) found that positively-framed appeals are more persuasive than negative ones when they have a promotion focus, whereas negativelyframed appeals are more persuasive than positive ones when they are prevention-focused.

\subsection{An Extant Model of the Effectiveness of Guilt Appeals}

In this research, the current knowledge on pro-social and charitable donation behaviour is synthesised into an extant model of guilt appeals, which serves as the foundation of the proposed model in health communications. It is accordingly noted that the factors focused on, and examined in, the extant model are not examined in this study.

A large number of research studies have examined the effects of guilt appeals in prosocial and charitable donation behaviour. Previous key findings in charity donations outlined that an ad's credibility (Cotte et al., 2005) and a moderate level of induced guilt (Coulter \& Pinto, 1995) are prerequisites for guilt appeals' effectiveness, and that empathy and self-efficacy are factors defining whether guilt or defensive reactions result (Basil, Ridgway, \& Basil, 2008). Among the mediators examined are: persuasion knowledge, including ad credibility, perception of manipulative intent (Coulter et al., 1999; Hibbert et al., 2007; Lwin \& Phau, 2010), and anger (Coulter \& Pinto, 1995); agent knowledge, including beliefs about the characteristics, competencies, and goals of the persuasion agent (e.g., the charity, Hibbert et al., 2007); perceived threats to unknown others, response-efficacy and self-efficacy (Lindsey, 2005); and responsibility (Basil, Ridgway, \& Basil, 2001, 2006).

Research on the effects of guilt appeals has also examined possible moderators such as an ad's credibility and perceived manipulative intent (Cotte et al., 2005), guilt characteristics such as types of guilt and levels of intensity (Giner-Sorolla, 2001; LaBarge \& Godek, 2006; Renner, Lindenmeier, Tscheulin, \& Drevs, 2013). Significantly, the moderating role of message tactics in motivating pro-social behaviour has also been examined, including message framing (i.e., loss versus gain) (Agrawal \& Duhachek, 2010; Duhachek, Agrawal, \& Han, 2012), and message sidedness (i.e., two-sided versus onesided) (Renner et al., 2013).

There is a differential effect in the emotional intensity between two types of anticipatory and reactive guilt. For example, Giner-Sorolla (2001) found that, in the case of a delayedcost dilemma activity (e.g., short-term positive but long-term negative consequence), 
anticipatory guilt is felt less intensely and less negatively than the reactive guilt that is experienced after people have committed the guilt-inducing action. The chance to avoid the transgression may cause reactive guilt, and also creates feelings of self-efficacy and self-control in anticipatory guilt. However, the failure to resist the temptation adversely increases the feelings of powerlessness and helplessness, and these overall contribute to negative effect. Furthermore, research has also shown that anticipatory guilt is perceived as the most adaptive among the three types (LaBarge \& Godek, 2006), because it is about the future and the potential that an individual can seek to avoid a negative emotion. In contrast, reactive guilt is viewed as more negative, because it involves past actions and transgressions that trigger action towards reducing that negative emotion.

These differences in the emotion aroused by anticipatory versus reactive appeals have some implications for the message persuasiveness. That is, consumers would respond more positively towards the ad and brand when ads used anticipatory compared to reactive appeals. Most interestingly, anticipatory guilt ads are processed heuristically rather than systematically, implying that consumers process and accept the ads with little appraisal (LaBarge \& Godek, 2006). That is because the feelings of self-efficacy and selfcontrol increased in anticipatory guilt appeals have positive short-term hedonic consequences for mood processing heuristically.

Recently, Renner et al. (2013) have further examined the effects of distinct types of guilt appeals (i.e., anticipatory versus reactive), reference groups (i.e., informational groups considered to have a special expertise in a field of interest versus non-informational groups not having this expertise), and message sidedness (i.e., two-sided versus onesided). They found that these variables influence the effectiveness of blood donation appeals.

Earlier research has demonstrated that the levels of induced guilt have different effects. That is, moderately intense guilt appeals are most effective, whereas highly intense guilt appeals tend to evoke anger, resentment and annoyance, leading consumers to respond negatively towards the message (Coulter \& Pinto, 1995; Moore \& Harris, 1996). This is consistent with the inverted-U relationship between an ad's intensity and its effectiveness derived from research in fear appeals (Bennett, 1996). Cotte et al. (2005) have also concluded that guilt appeals will have an adaptive effect on attitudes towards the brand if consumers do not believe the message to be manipulative. However, in contrast to Cotte 
et al.'s (2005) conclusions, in a charitable donations study Lwin and Phau (2014) found that the relationship between existential guilt and donation intentions was not moderated by manipulative intent. An explanation is that people may have high tolerance towards existential guilt appeals. That is, due to consumers' persuasion knowledge (Friestad \& Wright, 1994) and agent knowledge of the charity, consumers view existential guilt appeals as appropriate for charitable ads. It is therefore suggested that people have higher intensity thresholds to existential guilt, and this may extend Coulter and Pinto's (1995) investigation that appeal intensity thresholds vary across the three types of guilt appeals.

For the above-discussed mediating and moderating factors influencing the effectiveness of guilt appeals, an extant model is summarised (see Figure 2.1).

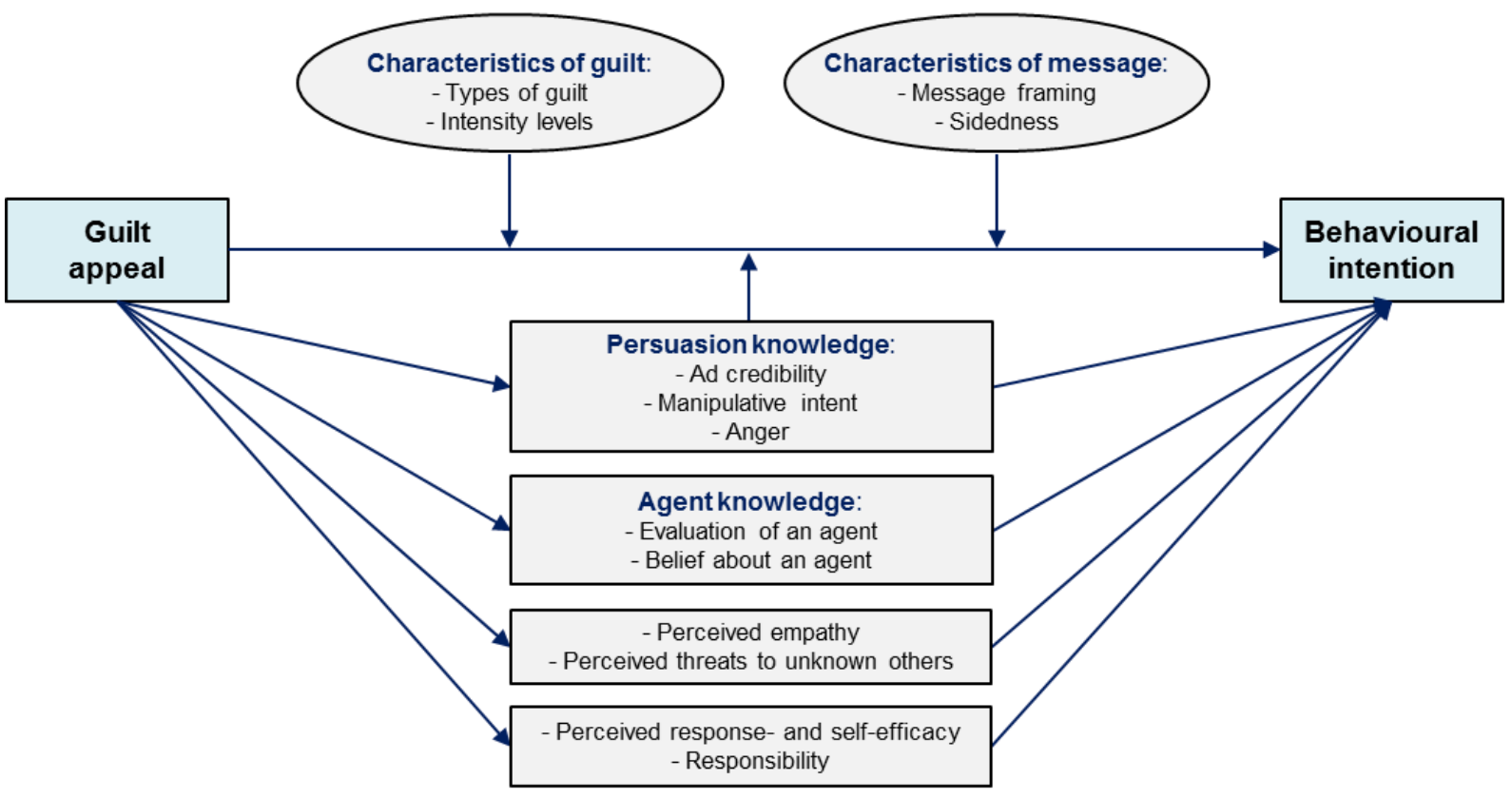

Figure 2.1. Extant Model of the Effectiveness of Guilt Appeals

The extant model specifies the relationships between guilt appeals and behavioural intentions mediated and moderated by several message characteristics factors. However, the model has largely neglected the role of significant individual difference factors and, more importantly, coping responses to the arousal of guilt that underlie behavioural intentions and are influential in predicting the effectiveness of such appeals. Further, the pro-social or charitable donations context differs from the health communications context such that, at the individual level, the former involves motivating people for the benefit of others while the latter involves stimulating people for the benefit of themselves. As a result, in the health communications context, guilt or shame appeals may operate 
differently due mainly to the interaction between the self-referential type of such emotions and the personal significance in the context. In light of these gaps, this study proposes a better model of the guilt and shame appeals' effectiveness for health communications.

\subsection{A Proposed Model of Guilt and Shame Appeals on Health Communications}

Based on the previously reviewed theoretical foundations and empirical studies in prosocial and donation behaviour focusing on guilt or/and shame messages, an extended model in heath communications incorporating a number of influential variables is proposed. These include distinct emotional arousals, coping response (identified as mediators), self-construal, regulatory focus, and personal cultural orientation (moderators). As previously mentioned, in health communications, the encounters have a personal significance to the receivers, therefore these variables are examined in consideration with the interaction of the self-conscious emotions of guilt and shame and the personal significance in the health communications context.

\subsubsection{Emotional Appeals versus Emotional Arousals}

An emotional appeal is a communication stimulus that attempts to evoke an emotional response. For example, guilt is an actual emotional response evoked from a guilt appeal that shows some type of outcome that the receiver wants to avoid. The distinction is important because previous research has often examined what were labelled 'levels of appeal' but, in fact, were different degrees of threat portrayed or types of threats (Boudewyns et al., 2013; LaTour \& Rotfeld, 1997). In addition, many studies did not examine the influence of fear (e.g., arousal) on consumer responses to threatening communications, but rather the impact of different types of appeals to fear on audience segments (e.g., based on the notion that different people fear different things) (LaTour \& Rotfeld, 1997). Thus, a clarification of arousals versus appeals affords a starting point for this research.

Notably, in the case of guilt and shame appeals, some authors suggested that appeals designed to elicit highly intense levels of guilt may actually evoke shame feelings unintentionally (Bennett, 1998; Boudewyns et al., 2013). For example, Boudewyns et al. (2013) claimed that the highly intense guilt appeals that lead to anger actually evoked a combination of shame and guilt, or shame only. For that reason, they concluded that 
shame-free guilt is more persuasive than guilt-free shame as the former is not correlated with anger while the latter is associated with anger.

Also, the interplay between the different emotions is important as it can determine the effectiveness of the message (De Pelsmacker, Cauberghe, \& Dens, 2011; Dillard \& Nabi, 2006). This might explain the inconsistent findings in the threat appeal driving literature that reflect a disconnect between emotion (e.g., fear) and behaviour (e.g., driving) (Carey, McDermott, \& Sarma, 2013; Carey \& Sarma, 2011). Therefore, this research examines guilt and shame separately through specific emotional arousals rather than appeals to control unintentional emotions, other than guilt or shame, evoked by the appeals to more fully understand the link between distinct emotion and behaviour.

Insight into the nature of emotional arousal resulting from emotional appeal provides the primary basis for understanding the phenomenon. While strong evidence exists to suggest that guilt and shame arousals are effective under the right conditions, based on findings to date, there is little evidence to confirm that they consistently work. By examining distinction of cognitive and emotional responses stemming from guilt relative to shame arousals, this study also makes findings consistent and generalisable.

\subsubsection{Coping Responses}

Guilt and shame appeals are persuasive messages that have the ability to create emotional responses which, in turn, cause coping responses (Agrawal \& Duhachek, 2010; Duhachek et al., 2012). The feeling of guilt or shame is an aversive experience, and it motivates an individual to try to ignore or avoid it (Czub, 2013). As a result, regulating emotional experiences of guilt or shame consists of undertaking various actions that aim at alleviating the current negative feelings and/or preventing experiencing guilt or shame in the future (for emotion regulation, see Gross, 1998). Broadly, there are adaptive and maladaptive coping responses to these emotional arousals. When emotional responses induce individuals to recognise a threat and search for ways to deal with, it is considered adaptive coping; in contrast, when individuals find ways to avoid the notion of threat, this is referred to as maladaptive coping (Lazarus, 1991b; Schoenbachler \& Whittler, 1996).

Specifically, to take the example of shame regulation, according to Czub (2013) an antecedent-focused strategy may be to give up a behaviour that the person knows may cause shame, such as adjusting the behaviour to the sociocultural rules and standards that 
condition social acceptance (adaptive coping), or in a defensive withdrawal from undertaking goals, challenges, or social contacts (maladaptive coping). A responsefocused strategy may be openly discussing, in a friendly environment, the situation in which shame has been activated, or simply leaving the situation in which shame has been felt, without denying what happened, and waiting until the emotion disappears on its own (adaptive coping), or through substitution of shame with anger (maladaptive coping).

In the extant fear literature, this distinction has been overlooked by researchers (e.g., Tanner, Hunt, \& Eppright, 1991); however, how broader emotional responses impact on coping responses has not been investigated. In particular, there is no detailed discussion regarding how emotions affect these coping responses through emotional intensity levels. To fill this theoretical gap, this research investigates the role of levels of emotional arousal in influencing coping responses to fully understand the processing of health emotional appeal messages.

The coping response to a negative emotional message can be influenced by the intensity of emotional responses aroused in the individual. Many studies on persuasion show that people in a negative mood state guard their negative mood against further intensity and resist negative self-evaluation (Agrawal \& Duhachek, 2010; Agrawal et al., 2007; Dickinson \& Holmes, 2008). For example, Dickinson and Holmes (2008) found that higher emotional responses based on the summed score across disgust, guilt, stress and anger did not lead an individual to respond adaptively. This inverse-U effect was commonly found in fear appeals research (Bennett, 1996), but interestingly was not obtained in existential guilt appeals on donations (Lwin \& Phau, 2011, 2014). In an antidrinking study, Agrawal and Duhachek (2010) added that compatible appeals (e.g., ads that evoke the same emotion) are less effective in influencing behaviour and intentions due to a process where people reduce the notion that they may cause the negative consequences featured in the message. Such defensive processing of compatible messages is triggered by a drive to reduce the existing negative emotion that is explained by an emotional overload account. Accordingly, compatibility or intense emotion results in a negative emotional overload that drives people to shut down and avoid processing the message (Agrawal et al., 2007). Therefore, the leading proposition presented in this research is that the coping responses to guilt and shame arousals vary depending on the intensity of the emotional feelings aroused by the receivers. 


\subsubsection{Self-Referencing}

In the literature, information about the self is one of the best-developed and most rich networks in people's memory (e.g., Symons \& Johnson, 1997) as it is highly structured, highly elaborate, and regularly accessed. Self-referenced information is more easily connected to earlier stored information, as the self is a regularly accessed construct in memory (Block, 2005). Because of these exceptional characteristics, self-referenced information has greater memory and persuasion advantages, and this is called the selfreference effect. Self-referencing is defined as a strategy of cognitive processing where audiences reference their individual self-structures when evaluating message information (Burnkrant \& Unnava, 1995). An example of self- and other-referenced guilt appeals can be seen in Block's (2005, p. 2031) studies. The self-referenced guilt appeal is as follows: "The more you drink, the more coordination you lose. That's a fact, plain and simple. Still you drink too much and then go out and expect to handle a car. When you drink too much, you can't handle a car. You can't even handle a pen. If you drink and drive, you risk losing everything". The other-referenced guilt appeal replaces the word 'you' with 'people' (i.e., "The more people drink, the more coordination they lose...”).

The self-reference effect has been examined in numerous domains and it has prevailed in the majority of studies (Symons \& Johnson, 1997), but there have been very few studies exploring circumstances in which the self-reference effect is accentuated or attenuated. Lord's (1980) study on self-referencing versus other-referencing across different modes of processing is cited as a notable exception to the self-reference effect. The findings suggested that the self could not have superior memory capacities for imagined, as compared to propositional, representations of the self. In this case, the self-image is less effective in memory than an other-image because of perceptual salience. Obviously, one imagines the scene from his/her physical perspective (e.g., looking out through one's own eyes), s/he becomes the non-salient figure while others become the salient figure in the imagined incident. Thus, the self-reference effect is reversed for events where they require someone to imagine a hypothetical incident or action happening to them (for more studies on the imagination hypothesis, see Brown, Keenan, \& Potts, 1986). Further, a study by Sedikides and Green (2000) showing more shallow processing and less recall for negative self-referent than other-referent material supports the speculation that the selfreference effect is reversed when information is negative. 
Based on the discussion above, it can be theorised that the effects of guilt and shame appeals on health message processing are varied by self-referencing. In health situations, such self-conscious emotional appeals work by making people imagine or anticipate a negative consequence. Additionally, according to the Cognitive-Motivational-Relational Theory of Emotion (Lazarus, 1991a, 1991b), the self versus other stake in health encounters has an impact on the appraisal of these self-conscious emotional appeals. It could be expected that self-referencing and the negative anticipated consequent emotions interact to make such guilt and shame appeals have significant effects on health message processing.

\subsubsection{Sources of Evaluation}

Guilt and shame both have internal and external sources of evaluation (Brommersma, 2007; Gilbert et al., 2007; Kim et al., 2011). Guilt is not just evoked internally by the self's action but, as Baumeister, Stillwell, and Heatherton (1994) explained, it may also be aroused externally through social interactions between people. For example, in the internal guilt, the transgressor is the only person who knows of its breach of conduct (i.e., the self's observation). The external guilt occurs when a transgression is known to others (e.g., friends, family members, i.e., the others' observation). Similarly, shame has two distinct internal versus external forms (Gilbert, 1998). Accordingly, while internal shame is related to experiences in which the self is devalued in one's own eyes and self-identity is harmed, external shame is associated with beliefs that others look down on the self and see the self as inferior, inadequate, disgusting or weak in some way.

Such internal and external sources of evaluation of guilt and shame have great relevance to how people process health messages. According to the Cognitive-MotivationalRelational Theory of Emotion (Lazarus, 1991a, 1991b), the source of evaluation has an impact on cognition of promoted issues due to the personal significance. Indeed, research on guilt appeals in charity shows that the others' presence (e.g., external guilt) would make salient a pro-social norm (Basil et al., 2001, 2006). For example, Basil et al.'s (2001) study demonstrated that the impact of guilt appeals on charitable donations is mediated by both a sense of responsibility and the activation of altruistic norms. Because the behaviour is influenced partly by society's expectations, the others' presence thus makes a pro-social norm salient. The effect of the presence of others on pro-social behaviour is not only involving what others will think but also serves to enhance the 
salience of norms people hold, which leads them to a sense of responsibility towards those norms (Basil et al., 2006). These results provided preliminary insight into the process through which internal versus external guilt appeals can generate pro-social intentions.

It is assumed that evaluation sources of guilt and shame may have an impact on health message processing; specifically, they vary in the emotional intensity aroused by these appeals. Although the impact of different evaluation sources of guilt and shame in psychopathology has received attention (Goss \& Allan, 2009; Grabhorn et al., 2006; Lee et al., 2001; Proeve \& Howells, 2002), there has been little research devoted to this role in health communications. This research examines the role of evaluation sources of guilt and shame in levels of emotional arousal intensity, which, in turn, may influence health message compliance.

\subsubsection{Self-Construal}

Studies on self-construal of independence versus interdependence have indicated that this has an impact on emotional message processing (Lee, Aaker, \& Gardner, 2000). More specifically, self-construal has the interactive effect with self-referencing on selfconscious emotional appeals' processing (Aaker, 2000; Aaker \& Williams, 1998; MurrayJohnson et al., 2001; Williams \& Aaker, 2002). For example, Aaker and Williams (1998) examined the persuasive effects of ego-focused (e.g., pride) versus other-focused (e.g., empathy) emotional appeals across cultures. They found that the self-reference effect was obtained for independent self-construals who viewed an ego-focused emotional appeal. That is, the ego-focused appeal created more favourable brand attitudes than did the other-focused appeal, the reverse pattern for independent self-construals who viewed the other-focused emotional appeal. However, for interdependent self-construals, the selfreferenced and other-referenced appeals were equally favourable across both kinds of emotions. Likewise, Block's (2005) study on the persuasiveness of fear and guilt appeals suggested that for individuals with interdependent self-construals, self-referenced versus other-referenced appeals are equally persuasive. For people with independent construals, the self-reference compared with other-reference effect is either advantageous or disadvantageous depending on the types of emotional arousals (e.g., fear versus guilt). Notably, the self-construals in these studies are measured by ethnicity (American versus Asian). 
Given the cultural focus of much research regarding self-construals, many researchers have argued that the self-construals are dynamic individual characteristics rather than stable cultural characteristics (Levinson et al., 2011; Martin et al., 2013). Therefore, the current research examines self-construal as an individual-level variable and its impact on levels of emotional arousal intensity. It is proposed that, for independents, selfreferencing is advantageous when the guilt is aroused as the emotion of internal sanction, and disadvantageous when shame is aroused as the emotion of social sanction; whereas for interdependents, self-referencing has no effect.

\subsubsection{Regulatory Focus}

There may be other individual difference characteristics that affect levels of guilt and shame arousal intensity (LaBarge \& Godek, 2006). Particularly, Regulatory Focus Theory (Higgins, 1997, 1998) has been suggested as a possible variable of note. A growing literature has demonstrated that a promotion versus prevention focus powerfully predicts cognition and affect (Aaker \& Lee, 2001). In fact, many studies found the impact of these distinct motivational patterns on the cognitive processes (Crowe \& Higgins, 1997), emotional responses (Higgins, Shah, \& Friedman, 1997), and behavioural intentions (Higgins, Roney, Crowe, \& Hymes, 1994).

Regulatory focus can be conceptualised as the message or viewers' regulatory focus. The message's regulatory focus has received much attention, but the viewers' regulatory focus has been the subject of few studies, and its potential role in the self-conscious emotional appeals effects is not clearly understood. Indeed, many studies focus on the message's regulatory focus, such as message framing (e.g., gain/approach versus loss/avoidance) (Duhachek et al., 2012; Kees, Burton, \& Tangari, 2010; Lee \& Aaker, 2002; Zhao \& Pechmann, 2007), but relatively few studies examine regulatory focus as an individual difference variable (e.g., viewers' regulatory focus) (Lockwood, Jordan, \& Kunda, 2002; Zhao \& Pechmann, 2007). More importantly, no studies on viewers' regulatory focus have examined the effects of the persuasive message regarding its antecedent influential components, such as the amount of affect (e.g., emotional arousal intensity level). This research will investigate viewers' regulatory focus (prevention- versus promotionfocused) in influencing levels of emotional arousal intensity elicited from guilt and shame appeals. It could be expected that both these negative emotional appeals will be felt more intensely by prevention- than promotion-focused people, because guilt and shame appeals 
focus on the painful emotion experienced on either contemplating or actually committing a failure.

\subsubsection{Personal Cultural Orientation}

The impact of culture on emotions experienced and regulated is widely accepted. Specifically, the impact of cultural norms on emotions experienced is weakened or strengthened depending on the centrality of the self in the emotion that is under scrutiny (Scollon et al., 2011). Generally, the amount of cultural differences is greater particularly for self-conscious emotions in comparison to other emotions due to the function of selfconsciousness in the emotions. Self-consciousness in these emotions has important social functions in helping people create and maintain relationships in a dynamic changing social hierarchy setting (e.g., group or dyadic) by providing feedback about their selfidentities (Goetz \& Keltner, 2007; Keltner \& Haidt, 1999). Due to differences in the social hierarchical structures, and differences in the function of self-consciousness, the cultural differences in self-conscious emotions are likely to be greater. For example, the feelings of pride focus on personal achievement and one's uniqueness apart from others, thus they are more congruent with the self of people in individualist cultures such as North America. Conversely, feelings of guilt facilitate social harmony and stimulate individuals to make reparations after transgressions, and are therefore more congruent with the self of people in collectivist cultures such as East Asia (Kitayama, Markus, \& Kurokawa, 2000; Kitayama et al., 2006).

In particular, the literature suggests that the valuation, elicitation, and behavioural consequences of guilt and shame differ across cultural contexts (e.g., Lee \& Paek, 2014; Wong \& Tsai, 2007). Accordingly, in societies that promote an independent self (e.g., individualism), there are obvious distinctions between the two emotions guilt and shame and guilt is perceived more positively than shame. However, in societies that promote an interdependent self (e.g., collectivism), there are less obvious distinctions between guilt and shame and shame is perceived more positively than guilt (Wong \& Tsai, 2007). Indeed, they are experienced even when transgressions are committed by others, such as reflected shame when one brings shame to one's family or community in these cultures. Significantly, in collectivist cultures, experiencing shame has an association with adaptive consequences. This is because selves are contextually and situationally dependent, and therefore situational changes in concepts of the self are viewed as normal (Kondo, 1990). 
For example, in cultures influenced by Confucian values where people are encouraged to constantly nurture and improve themselves, changes in the self are explicitly appreciated and expected (Cho, 2000). Hence, feeling bad about the self is not merely normal, but to some extent expected since it serves the greater goal of self-improvement. This implies that the effects of guilt and shame arousals on behavioural intentions may vary across cultures. However, most studies of guilt and shame across cultures have been tested based mostly on nationality/ethnicity. Thus, this study explores under which individual culture (i.e., personal cultural orientation) guilt or shame is most effective.

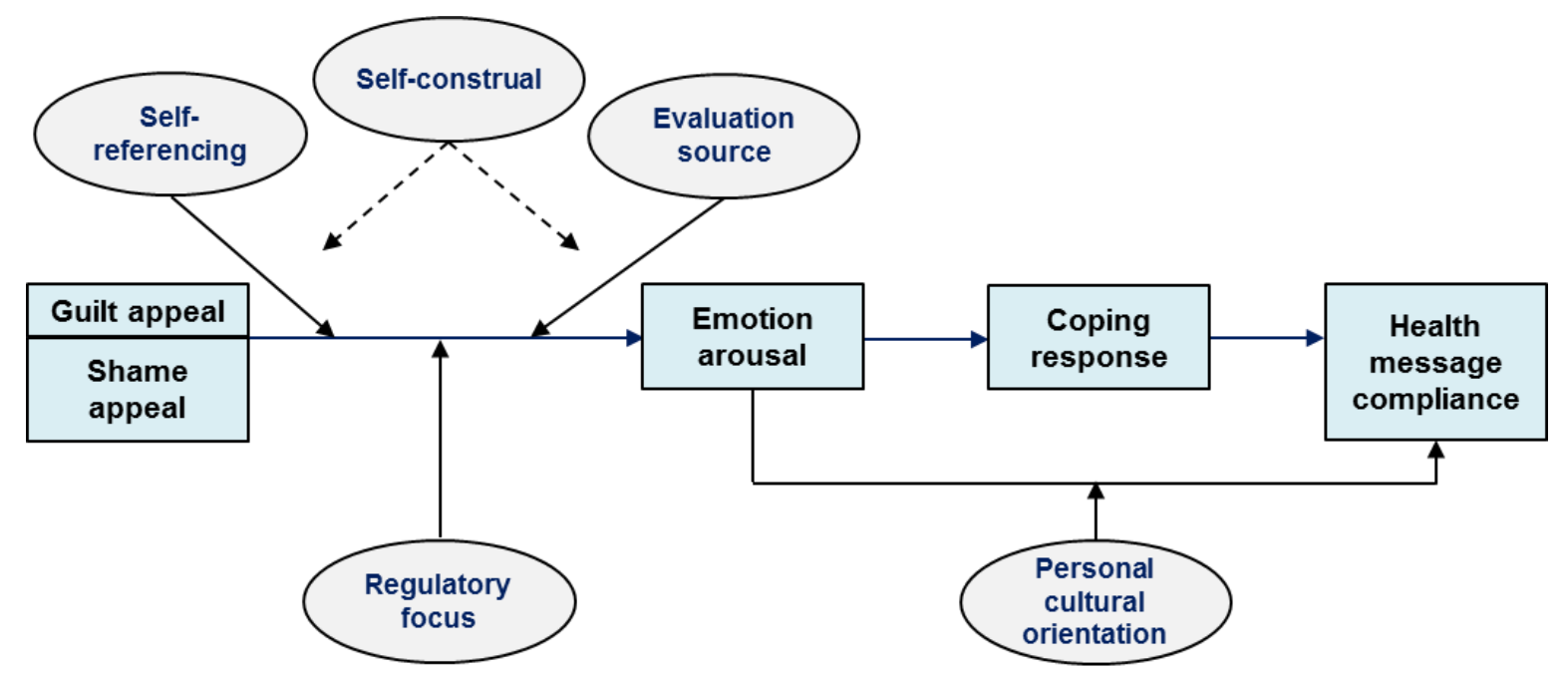

Figure 2.2. Proposed Model of the Effectiveness of Guilt and Shame Appeals on Health Communications

From the aforementioned discussions, a model for health communications of the effectiveness of guilt and shame appeals is proposed (see Figure 2.2).

\subsection{Chapter Summary}

Considering the reviewed literature, limited empirical research, and the identified gaps, it would be of interest to build and test an extended model for better understanding the processes by which guilt and shame appeals may lead to compliance with health messages. The gaps evident in the extant research include: $(i)$ the role of coping response to emotion as a mediator which specifies the underlying dimension that accounts for health message compliance; (ii) the impacts of the degree of self-construal and regulatory focus of individuals on guilt and shame arousals; (iii) the impact of personal cultural orientation on guilt and shame arousals' effects; and (iv) the lack of empirical research examining guilt and shame separately (Boudewyns et al., 2013). The lack of generalizable 
research in these areas may be a factor contributing to the differential effects of guilt and shame appeals and inverted U-shaped relationship in guilt appeals' effects.

Several opportunities for improvement within the extant literature were identified to aid the present study in closing the identified gaps. The study, therefore, examines the effects of the mediating factor of the coping response to emotion, the interactive effects of the dual moderating factors of self-referencing, the sources of evaluation with self-construal, and the additional individual difference factors of regulatory focus and personal cultural orientation. Taken together, this better predicts the effectiveness of guilt and shame appeals. Accordingly, the proposed model: $(i)$ incorporates coping response as the cognitive outcome of emotional arousal underlying health message compliance; (ii) explains the role of self-referencing and the evaluation sources of guilt and shame appeals interacting with self-construals, and individual difference regulatory focus in predicting the levels of emotional arousal intensity; (iii) explores under which personal cultural orientation guilt or shame appeals are most effective; and (iv) differentiates between guilt and shame appeals' effects in the context of health communications. Overall, these investigations at both mediating and moderating impacts offer a better understanding of the effectiveness of guilt in comparison to shame appeals on health communications.

It is also worth noting that, as mentioned in the extant model, the types of guilt appeals matter to message processing. There is a differential effect in the intensity with which types of anticipatory, reactive, and existential guilt are experienced (Giner-Sorolla, 2001; LaBarge \& Godek, 2006). That is, the anticipatory guilt is felt less intensely and less negatively than the reactive guilt experienced after engaging in guilt-inducing behaviour. Importantly, there is congruence between the appeal and product/service types. For example, congruence was found in charitable donations with reactive appeal and health behaviours with anticipatory appeal (LaBarge \& Godek, 2006). This research complied with the ethical consideration by using anticipatory guilt to examine the role of other factors in the guilt appeals' effects. As such, the anticipatory appeals of guilt are selected for the study.

Having established the relevant areas of literature, and identified the research gaps and objectives of the current research, the next chapter discusses in more detail, the possible relationships between the constructs and presents a conceptual framework for the study. 


\section{Chapter 3. Conceptual Framework and Hypotheses}

\subsection{Introduction}

A review of the literature recognised a lack of extensively theoretical and empirical study regarding the effectiveness of guilt and shame appeals in health communications. A conceptual framework was developed to empirically test the impact of the mediating factor of the coping response to emotion, the interactive effects of the moderating factors of self-referencing, the sources of evaluation with self-construal. In addition, the research considers the moderating effects of the individual difference factors of regulatory focus and personal cultural orientation. This chapter discusses each hypothesis in detail, within the framework, which was developed based on the literature as reviewed in Chapter 2, followed by a presentation of the complete conceptual model to be empirically tested in Chapter 4.

\subsection{Guilt and Shame Arousals}

\subsubsection{Guilt and Shame Appeals}

The intensity levels of emotional appeal have been used in examining the effects of threat communications (e.g., Dickinson \& Holmes, 2008; Tanner et al., 1991). The three main levels of threat most regularly used in a threat appeal communication are low, moderate and high (Schoenbachler \& Whittler, 1996). The main point of discussion among these studies is the inverse- $U$ effect that advocates moderate threats to bring out the 'optimal level of threat'. The inverse-U effect suggests that, in terms of persuasiveness, moderate levels of threat outperform both high and low levels of threat and therefore should be considered as the 'optimal' level of threat. However, findings continue to be mixed (Cotte et al., 2005; Lindsey, 2005; Turner \& Underhill, 2012). In the case of guilt and shame appeals, Bennett (1998) suggested that messages designed to elicit highly intense levels of guilt may actually evoke shame feelings unintentionally. Similarly, Boudewyns et al. (2013) claimed that the highly intense guilt appeals that lead to anger actually evoked a combination of shame and guilt, or shame only. Thus, there is a need to control the impact of appeal-intensity levels to examine the effects of emotional appeals on respective emotional arousals through which persuasive message is processed.

In the charitable donations and pro-social behaviour literature, guilt and shame are most frequently researched. However, in such a different context, health communications, they 
are under-studied. As mentioned above, a study examining guilt and shame arousals rather than appeals in order to control the impact of these appeals' intensity to more fully understand the distinct emotional responses on message persuasiveness is more important. In threat appeal communications, the relationship between appeals and emotional arousals was found to be strong, regardless of emotion type (Carey et al., 2013; Dickinson \& Holmes, 2008). The findings of a meta-analysis suggest that threat appeals have a strong impact on the level of emotion aroused (e.g., fear) in individuals (Carey et al., 2013). In terms of the relationship between levels of threat and emotional arousal, moderate and high threats were able to elicit the strongest emotional response (a global emotional reaction including fear, sadness, anger, shame and disgust, Dickinson \& Holmes, 2008). Therefore, the link between guilt and shame emotional appeals and emotional arousals elicited is definitely strong. This leads to the subsequent hypothesis:

H1: The relationship between (a) guilt appeals and guilt arousals, (b) shame appeals and shame arousals is positive.

\subsubsection{Message Compliance}

The link between negative emotional arousal, attitude formation and behavioural compliance is theoretically supported (e.g., Brennan \& Binney, 2010). Thus, social marketers and public policy makers often use negative emotional appeals in an attempt to encourage compliant behaviour from the public (Han et al., 2014). Individuals frequently experience guilt or shame in daily life, stemming from engaging in unhealthy behaviour such as smoking, drinking driving or binge drinking. Health communications can feasibly arouse guilt and shame in individuals to motivate positive behaviour because of a threat posed to personal notions of self-integrity (Leary \& Baumeister, 2000; Sznycer et al., 2016).

However, there are mixed findings regarding negative emotions in general. Some fear studies found no relationship between emotional arousal and behavioural intention (Rogers, 1975; Schoenbachler \& Whittler, 1996), while others found that stronger emotional responses, regardless of emotion type, lead an individual to using an adaptive response (Folkman, Schaefer, \& Lazarus, 1979; Tanner et al., 1991) and, ultimately, message compliance (Ang \& Low, 2000). As noted previously, distinct guilt and shame are under-studied. This research examines the impact of guilt and shame emotions 
separately on message compliance [by through emotional arousals rather than emotional appeals].

\subsubsection{Guilt arousal}

Early studies have found the inverted U-shaped relationship in guilt intensity and message persuasion (Coulter et al., 1999; Coulter \& Pinto, 1995; Ghingold \& Bozinoff, 1982; O'Keefe, 2002; Pinto \& Priest, 1991; Pinto \& Worobetz, 1992). That is, highly intense guilt messages often cause anger and are less persuasive, while moderately intense guilt can elicit guilty feelings, thereby motivating people to change attitudes about a given product or offer. However, recent studies testing guilt messages in both the pro-social and commercial realms attest to the effectiveness of intense guilt levels (Cotte et al., 2005; Lindsey, 2005; Turner \& Underhill, 2012). Such that, they did not support the inverted-U effect but rather they supported a linear effect.

Research focused on charitable donations indicates that people who experience guilt tend to want to make reparations for the harm that has been done to others and want to avoid actions that might result in guilty feelings (Hibbert et al., 2007; Lwin \& Phau, 2011, 2014). Taken together, there is strong evidence to suggest that any intense guilt arousals can evoke positive behaviour. It is thus assumed that there is a strong positive relationship between guilt arousals and behaviour intentions.

\subsubsection{Shame arousal}

In comparison with guilt, shame tends to create more defensive responses (Abe, 2004; Lindsay-Hartz, 1984; Stuewig et al., 2010). However, in social psychology, shame essentially helps to motivate socially favourable behaviour and efforts towards selfimprovement (de Hooge et al., 2010; Gausel \& Leach, 2011; Lazarus, 1991a; Sznycer et al., 2016). In fact, shame is associated with a greater desire to change aspects of one's self implicated in a past transgression (Gausel \& Brown, 2012), activates approach action tendencies, manifesting as pro-social behaviour like directly repairing social errors (de Hooge et al., 2010), and deters actions that would lead to more devaluation than benefits (Sznycer et al., 2016). This claim is definitely supported in contexts that promote an interdependent view or a collectivist orientation (Bagozzi et al., 2003; Scollon et al., 2011; Silfver, 2007; Wong \& Tsai, 2007). It is thus hypothesised that there is a positive relationship between shame arousals and message compliance. 
Indeed, both guilt and shame appeals are particularly persuasive tools in health communications for reducing harmful behaviours, such as binge and underage drinking (Agrawal \& Duhachek, 2010). The self-conscious emotions of both guilt and shame carry extremely strong personal implications, therefore individuals have experienced these with health messages are highly motivated to make amends as a result of the personal significance in the message as well as a threat posed to personal notions of self-integrity (Leary \& Baumeister, 2000; Sznycer et al., 2016).

In the preceding discussions, the following hypothesis is proposed for both guilt and shame arousals towards health message compliance:

H2: The relationship between (a) guilt arousals and message compliance, (b) shame arousals and message compliance is positive.

\subsection{Coping Responses}

\subsubsection{Adaptive and Maladaptive Coping}

In health contexts, adaptive coping responses are those that relate to the intention of improving an individual's health (Schoenbachler \& Whittler, 1996), such as where an individual may rationalise that "Smoking negatively affects your health, therefore no one should smoke" (Dickinson \& Holmes, 2011, p. 122). In contrast, when an individual finds ways to avoid the notion of danger, this is referred to as a maladaptive coping response (Schoenbachler \& Whittler, 1996). A maladaptive coping response towards a smoking message may include statements such as "God will take care of people who smoke", "Smoking is only dangerous for old people", and "One cigarette won't have any effect" (Dickinson \& Holmes, 2011, p. 122). Individuals who engage in maladaptive coping responses are unlikely to accept health messages, and thus adaptive coping responses are sought since previous research has shown these responses result in positive attitudes towards the messages (Belch, Belch, \& Jones, 1995; Pechmann, 2001; Schoenbachler \& Whittler, 1996). When an adaptive coping response occurs, an individual has a positive attitude towards the messages so they will accept the advice/suggestions given in heath messages (Arthur \& Quester, 2004; Schoenbachler \& Whittler, 1996). Therefore, adaptive coping responses are associated with health danger removal (e.g., problem-focused coping), while maladaptive coping responses are associated with the control of one's own failure generated by the danger (e.g., emotion-focused coping). 
It is evident that there is a strong association between coping responses (e.g., adaptive versus maladaptive) and health message compliance. More specifically, adaptive coping responses elicited from a guilt or shame response are likely to result in message compliance, whereas maladaptive coping responses are unlikely to result in message compliance. This leads to the proposition that the coping response is mediating to message compliance.

\subsubsection{Mediating Emotional Arousal and Message Compliance}

The importance of understanding the role of arousal in emotional-appeal processing is based on the relationship between emotional arousal and coping response. The acceptance (adaptive coping response) or resistance (maladaptive coping response) towards a negative emotion-based message can be largely influenced by the emotional response aroused, regardless of emotion type, in individuals (Dickinson \& Holmes, 2008; Schoenbachler \& Whittler, 1996).

As mentioned, guilt and shame are negative self-referential emotions which people who have experienced these are highly motivated to make amends (Agrawal \& Duhachek, 2010; Leary \& Baumeister, 2000; Sznycer et al., 2016). It is thus assumed that strong intensity emotional arousals, regardless of guilt or shame, are more likely to lead to adaptive processing. As such, it is expected that the higher the intensity emotional arousals, the greater the adaptive coping to the health message.

Based on the above discussions, the following hypothesis is suggested for both guilt and shame arousals:

H3: Coping responses will mediate the relationship between (a) guilt, (b) shame arousals and message compliance.

\subsection{Varying Guilt and Shame Arousal Intensity}

\subsubsection{Regulatory Focus}

Studies demonstrate that matching viewers' regulatory focus with that of the message's could be beneficial (Aaker \& Lee, 2001; Zhao \& Pechmann, 2007). That is, promotionfocused viewers are more persuaded by health messages that suggest behaviour changes lead to achievements (e.g., promotion-focused framing: "If you do not smoke, you can obtain positive results, such as..."), whereas prevention-focused viewers are more 
persuaded by health messages suggesting that behaviour change results in threat reduction (e.g., prevention-focused framing: "If you do not smoke, you can avoid negative results, such as..."). However, the role of viewers' regulatory focus in influencing the intensity of negative emotional arousals from guilt and shame appeals is not clearly understood.

It is argued that promotion-focused individuals are motivated by achievements and are sensitised to opportunities for advancement, while prevention-focused individuals are motivated to avoid threats to security and safety and are sensitised to occasions of threat. For guilt and shame, both are negative emotions, it could, therefore, be expected that individuals with a prevention focus will respond more intensively to these negative emotional appeals than individuals with a promotion focus. This is because both negative self-conscious guilt and shame emotional appeals focus on the threats to the committer's notions of self-integrity. Thus, it is hypothesised that:

H4: Levels of emotional arousal elicited from (a) a guilt appeal or (b) a shame appeal are impacted by individuals' regulatory focus. Specifically, preventionfocused individuals will exhibit higher guilt or shame arousal than their promotionfocused counterparts.

\subsubsection{Self-Construal}

Culturally, there are strong differences in self-conscious emotions that reflect on the selfawareness and self-evaluations (Eid \& Diener, 2001; Tangney \& Fischer, 1995; Weiner, 1986). A number of theoretical works characterise American culture as a 'guilt culture' and Japanese culture as a 'shame culture' (Benedict, 1946; Creighton, 1990). Creighton (1990) states that the Japanese people's experience of shame is driven by threat that their inadequacies will result in the loss of union or exclusion from the group as they are concerned with maintaining social bonds. However, shame is an inadequate social sanction for American people given motivations of separateness and self-reliance. Consistently, shame is more elaborated (Li, Wang, \& Fischer, 2004) and more commonly used to teach moral lessons (Tinsley \& Weldon, 2003) in China than America. Also, selfreports of shame-proneness are higher among Asian-Americans than EuropeanAmericans (Lutwak, Razzino, \& Ferrari, 1998).

While self-construal is imposed by culture (the independent self is emphasised more in individualist cultures, while the interdependent self is represented more often in 
collectivist cultures, Cross, Hardin, \& Gercek-Swing, 2010), self-conscious emotions are sensitive to self-construal differences. Indeed, research suggests that a different selfconstrual - how individuals define themselves with respect to others (Markus \& Kitayama, 1991; Singelis \& Sharkey, 1995) - leads to different self-conscious emotions. This is because these emotions rely on self-awareness and self-evaluations (Tracy \& Robins, 2007a, 2007b). As a result, intensity levels of self-conscious emotional feelings vary depending on the centrality of the self in the self-conscious emotions and selfconstruals.

Previous findings have shown that self-construal plays a moderating role in predicting moral behaviour between two different ego-focused and other-focused emotions (Kim \& Johnson, 2013, 2014). For example, Kim and Johnson's (2014) studies investigating a moderating effect of self-construal on moral judgement revealed that independents were more likely to be influenced by the feeling of pride (i.e., ego-focused) than shame (i.e., other-focused). Conversely, interdependents were more likely to be influenced by the feeling of shame than pride with respect to counterfeits when they evaluated the rightness or wrongness of the purchase of counterfeits. However, research on self-construal differences in a discrete emotion arousal intensity, especially guilt or shame, is lacking.

\subsubsection{Guilt appeals}

Theoretically, collectivist cultures (e.g., Japanese, Chinese) favoured shame emotion (shame culture) while individualist cultures (e.g., European, American) favoured guilt emotion (guilt culture) (cf. Mead, 1971). This is because in individualist cultures, people rely on an internalised sense of right and wrong to control behaviour; collectivist cultures, on the other hand, rely on people's concerns about how they look to others (Sabini \& Silver, 1997). Empirically, Eid and Diener (2001) studied norms for experiencing emotions in different countries and showed that there is a tendency for people in the more individualist countries (i.e., America, Australia) to feel negative emotions (e.g., anger, fear, sadness, guilt) more intensely than do people in the more collectivist countries (i.e., China, Taiwan).

Research in self-construals implies that additional manifestations of prevailing motivational tendencies for maintaining positive distinctiveness for independent selfconstruals and maintaining social connectedness for interdependent self-construals are the attributions that elicit guilt and shame emotions (Dean \& Fles, 2016). Individually, 
independent self-construals are expected to attempt to be unique, to promote their own personal goals, more sensitive to personal responsibility, and independent from others (Markus \& Kitayama, 1991; Singelis \& Sharkey, 1995). It is assumed that in a guilt appeal, an internalised sense of moral responsibility is the salient feature. Since independent people feel a greater sense of right and wrong, a guilt appeal making their own personal responsibility/accountability more salient will create an elevated response, compared to interdependents. Thus, it can be expected that with exposure to the same guilt appeal, independent self-construals, as an independent entity, will experience the emotion more intensively. As a result, it can be postulated that:

H5 (a): For guilt appeals, independent self-construals will exhibit higher arousal than their independent counterparts.

\subsubsection{Shame appeals}

Previous researchers argued that cultural values may have a different influence on the reported experiences and behavioural expression of shame (Lee \& Paek, 2014; Wong \& Tsai, 2007). They claim that these negative views might be associated with cultural beliefs, such as matters for saving face and family honour (the salient feature in collectivist cultures) that could otherwise induce strong feelings of shame (Gilbert, Gilbert, \& Sanghera, 2004; Lam, Tsang, Chan, \& Corrigan, 2006; Lam et al., 2010). Further, the frequency of occurrence, and even the valence, of shame emotion vary across cultures (Menon \& Shweder, 1994; Tracy \& Robins, 2007a; Wong \& Tsai, 2007). For instance, shame is perceived to be appreciated in collectivist societies rather than in individualist ones, because it reaffirms the individual's place and sense of belonging in one's own social group (Menon \& Shweder, 1994; Wong \& Tsai, 2007). Likewise, Mesquita and Karasawa (2004) note that while shame is one of the most aversive emotions in an individualist culture, shame in a collectivist culture creates social harmony. Experiencing shame, the transgressor implicitly acknowledges his/her inferiority and embarrassment against the important social rules and expectations, and thus commits to improving their behaviour in the future.

Given that people with a chronic and salient interdependent view readily accept negative self-relevant information and use it as the basis for future self-improvement (Heine et al., 2001), interdependent self-construals are expected to be concerned with ongoing relationships, to maintain interdependence, to perform their part of group actions on the 
job, to adjust to, and fit into, their groups and relationships and, in general, to promote group welfare. When shamed, interdependents become aware that their actions or performances are negatively evaluated and come to feel that their self has been degraded and ridiculed. It would be argued that with exposure to the same shame appeal, interdependent self-construals might experience shame more intensively.

H5 (b): For shame appeals, interdependent self-construals will exhibit higher arousal than their independent counterparts.

\subsubsection{Self-Construal Interacting with Self-Referencing and Sources of Evaluation}

\subsubsection{Guilt appeals}

\section{Self-referencing}

Research notes that the relative intensity of guilt may vary depending on determined conceptions of self-reference (Block, 2005; Meyers-Levy \& Peracchio, 1996). That is, individuals who view a self-referenced guilt appeal involving an unpleasant, negative health-related event, and are told that they are responsible for its occurrence, will experience guilt more intensely compared with an other-referenced appeal involving others' responsibility as guilt is the emotion of internal sanction (Block, 2005). Further, whether self-referenced emotional appeals are more or less aroused may also depend on individuals' independence versus interdependence (e.g., self-construal) (Aaker, 2000; Aaker \& Williams, 1998; Murray-Johnson et al., 2001; Williams \& Aaker, 2002). It is therefore proposed that not only do self-referenced guilt appeals arouse guilt more intensively than do other-referenced guilt appeals, but this effect is also influenced by independent or interdependent self-construal.

Block's (2005) study on the persuasiveness of fear and guilt appeals indicated that for individuals with independent construals (measured by ethnicity, i.e., America versus Asian), the self-reference versus other-reference effect is obtained depending on the types of emotional arousals (e.g., fear versus guilt). It is thus suggested that, for people with independent self-construals, the self-reference effect is either advantageous or disadvantageous depending on the centrality of the self in the emotion that is elicited from a health message. Attributions of causality to the self versus the other in the independents create different levels of perceived guilt. At least one study reported that people who have independent self-concepts felt guilty when they recognised they had violated their own 
personal standards, while people who have dependent self-concepts felt guilty when they recognised that their wrongdoings hurt others (Stipek, Weiner, \& Li, 1989). Thus, it is argued that, for independents who perceive the self as a separate and distinct entity, the self-reference effect should be more prominent in perceived guilt than for people with interdependent self-concepts. The self-reference effect will accordingly be obtained when independents process the guilt appeals. Specifically, the self-referenced guilt appeals lead to more intense guilt than do the other-referenced appeals. Therefore, it can be hypothesised that:

H6 (a): For independent self-construals, levels of emotional arousal elicited from a guilt appeal are impacted by self-referencing. Specifically, they will exhibit higher guilt arousal for a self-referenced appeal than for an other-referenced appeal.

However, for people with interdependent self-construals, self-referenced guilt versus other-referenced guilt appeals are equally persuasive regardless of self-conscious emotional types (Aaker \& Williams, 1998; Block, 2005). Indeed, Aaker and Williams' (1998) studies on the persuasiveness of ego- (e.g., pride) versus other-focused (e.g., empathy) emotional appeals found that the self-reference effect was not obtained for interdependent self-construals across two emotions. That is, for individuals with interdependent self-construals, the self- and other-referenced appeal was equally favourable in terms of brand attitudes regardless of self-conscious emotional types. This means that self-referencing has no effect for those interdependent people who experience guilt. This leads to the following hypothesis:

H6 (b): For interdependent self-construals, levels of emotional arousal elicited from a guilt appeal are not impacted by self-referencing. Specifically, they will exhibit equal guilt arousal for a self-referenced appeal as for an other-referenced appeal.

\section{Sources of evaluation}

Research indicates that the impact of internal and external sources of evaluation for experienced guilt and shame on message processing is moderated by self-construal (Kim \& Johnson, 2013; Wong \& Tsai, 2007). According to Wong and Tsai (2007), an assumption of prevailing models of guilt and shame states that internal orientation (e.g., oriented to one's own standards/view) is more powerful and genuine than external 
orientation (e.g., oriented to others' standards/view) in the case of the self that is distinct, separate from others, and defined by stable personal characteristics, which is referred to as an independent self-construal. For independents, the internal source of evaluation should be more pronounced in perceived guilt and shame than the external one. For guilt, the internal source of evaluation will be more powerful when independent self-construals process the appeals. That is, the internal guilt appeals lead to more intense guilt than do the external guilt appeals. Thus:

H7 (a): For independent self-construals, levels of emotional arousal elicited from a guilt appeal are impacted by the evaluation source. Specifically, they will exhibit higher guilt arousal for an internal appeal than for an external appeal.

However, for interdependents, internal versus external sources of evaluation for guilt appeals are equally persuasive, meaning that the source of evaluation has no effect for those people because individuals with interdependent perceptions of the self view themselves in terms of their connections with others, external influences (e.g., other people's thoughts and feelings) are as important and meaningful as internal ones (e.g., one's own thoughts and feelings) (Wong \& Tsai, 2007). This means that the source of evaluation has no effect for interdependent people who feel guilt. Therefore, it can be hypothesised that:

H7 (b): For interdependent self-construals, levels of emotional arousal elicited from a guilt appeal are not impacted by the evaluation source. Specifically, they will exhibit equal guilt arousal for an internal as for an external appeal.

\subsubsection{Shame appeals}

\section{Self-referencing}

As noted previously in Section 2.5.3. Self-referencing, both imagining oneself and receiving aversive information diminishes the likelihood of the self-reference effect (Lord, 1980; Sedikides \& Green, 2000). As Lord (1980) reported, the self could not have superior memory capacities for imagined representations of the self as compared with propositional representations of the self. In this case of anticipated shame, the otherimage (i.e., other-reference) is more effective in memory for the self than the self-image (i.e., self-reference) because of perceptual salience. Besides, the negative valence of shame appeals also reduces the effect of self-reference. Since a shame appeal in health 
messages relies on the social sanction of an imagined negative consequence to the self, the mentioned reversed self-reference effect has suggested that self-referenced shame appeals would be less influential than other-referenced for people with independent selfconstruals.

Taken together, the self-reference effect could be reversed when people with a primarily independent view of self, process an anticipated shame appeal. Thus it could be argued that, for independent self-construals, the other-referenced shame appeals lead to more intense shame than do the self-referenced. As a result, the following hypothesis is suggested:

H8 (a): For independent self-construals, levels of emotional arousal elicited from a shame appeal are reversely impacted by self-referencing. Specifically, they will exhibit higher shame arousal for an other-referenced appeal than for a selfreferenced appeal.

As previously mentioned with respect to self-referencing, regardless of either guilt or shame emotional types, for people with interdependent self-construals, self-referenced versus other-referenced shame appeals are equally persuasive (Aaker \& Williams, 1998; Block, 2005). This means that self-referencing has no effect for interdependent people who feel guilt or shame. It could be argued that as a blurred self-perception, interdependent self-construals will arouse shame equally with exposure to self-referenced versus other-referenced shame appeals. The following hypothesis is therefore generated:

H8 (b): For interdependent self-construals, levels of emotional arousal elicited from a shame appeal are not impacted by self-referencing. Specifically, they will exhibit equal shame arousal for a self-referenced appeal as for an other-referenced appeal.

\section{Sources of evaluation}

As with guilt, the effect of internal and external sources of evaluation for experienced shame on message processing is affected by self-construal (Kim \& Johnson, 2013; Wong $\&$ Tsai, 2007). Accordingly, the self with independent views evaluates the internal source of shame as more powerful and genuine than the external one. For independents who are predisposed to viewing the self as a separate and distinct entity, the internal source of 
evaluation should be more pronounced in perceived shame than the external one. For shame, the internal source of evaluation will thus be more powerful when independent self-construals process the appeals. That is, the internal shame appeals lead to more intense shame than do the external shame appeals. As a result, it can be proposed that:

H9 (a): For independent self-construals, levels of emotional arousal elicited from a shame appeal are impacted by the evaluation source. Specifically, they will exhibit higher shame arousal for an internal appeal than for an external appeal.

However, as noted earlier, for individuals with interdependent perceptions of the self viewing themselves in terms of their connections with others, external influences are as important and meaningful as internal ones (Wong \& Tsai, 2007). This means that for these people, internal versus external shame appeals are equally persuasive. In other words, the source of evaluation has no effect for interdependent people who experience shame. It could be argued that as a dependent entity, the self cannot be separated from others or from the social context, interdependent self-construals will arouse shame equally when exposure to internal versus external shame appeals. Therefore:

H9 (b): For interdependent self-construals, levels of emotional arousal elicited from a shame appeal are not impacted by the evaluation source. Specifically, they will exhibit equal shame arousal for an internal appeal as for an external appeal.

\subsection{Under Which Personal Cultural Orientation Is Guilt or Shame Most Effective?}

Literature supports the claim that different emotions might enhance or impede persuasive effectiveness (Brennan \& Binney, 2010; Dillard \& Nabi, 2006; Dillard \& Peck, 2000). For example, Brennan and Binney (2010) conducted a qualitative study on compliance with income-reporting requirements and argued that fear, guilt, and shame may be differentially motivating. Specifically, an overuse of fear appeals can result more often in fight (e.g., aggression), shame appeals can result in flight from the message (e.g., social anxiety) and guilt appeals can be motivating. Consequently, it is necessary to carry out further research that compares the discrete emotions' effects on message persuasiveness. Following this, the current research investigates the differential effects of guilt and shame on health message compliance to determine under what circumstances which type of these two emotions is most effective. More specifically, this study examines the role of 
personal cultural orientation in guilt and shame emotional arousals in influencing message compliance in order to learn in which individual culture guilt or shame is most effective.

Across cultures, research has stated that guilt and shame lead to different action tendencies (Abe, 2004; Dearing et al., 2005; Kim et al., 2011; Lindsay-Hartz, 1984). Accordingly, arousing guilt has been shown to motivate reparative action and prompt behavioural change, whereas shame tends to motivate efforts to hide or disappear. For instance, Dearing et al. (2005) examined the relationship of shame-proneness (one's tendency to feel bad about his/her self) and guilt-proneness (one's tendency to feel bad about his/her specific behaviour) to substance abuse. They found that shame-proneness is generally positively correlated with alcohol and drug abuse, whereas guilt-proneness was inversely related (or unrelated) to problems with alcohol and drugs (particularly marijuana). Given these findings, the intersection of individual culture and guilt and shame emotions is increasingly an important but under-studied area. Further, most studies of guilt and shame across cultures were based on ethnicity as chronic national characteristics rather than personal cultural orientation as dynamic individual characteristics. Thus, this study explores in which personal cultural orientation these two self-conscious emotions will be up-regulated or down-regulated.

\subsubsection{Guilt}

At the national level, guilt is considered as a more favourable emotion in individualist cultures, resulting in a higher emotional arousal than in collectivist cultures, resulting in a lower emotional arousal (Eid \& Diener, 2001). However, in terms of emotion regulation, empirical studies found that there are not clear cultural differences in guilt feelings (Wallbott \& Scherer, 1995). Therefore, it is possible that coping with guilt is unrelated to cultural contexts. Meanwhile, in the dominant models of guilt, regardless of culture, guilt leads to reparative action (Wong \& Tsai, 2007). For example, empirical findings in the American contexts suggest that experiencing guilt leads to higher self-esteem and increases in empathy and perspective taking (Tangney, 1998). Taken together, feelings of guilt are likely to lead to message compliance for both individualist and collectivist people.

At an individual level, while there is a lack of studies on culture in guilt and shame feelings, personal cultural orientation is dominantly influenced by national culture 
(Alegria, Atkins, Farmer, Slaton, \& Stelk, 2010). This can lead to the following hypothesis.

H10: The impact of guilt arousal on message compliance is not moderated by personal cultural orientation. Specifically, individuals experiencing guilt are more likely to comply with the message, regardless of either (a) individualist or (b) collectivist orientations.

\subsubsection{Shame}

The influence of shame on coping responses and behavioural intentions depends on the values and beliefs associated with the given culture (Czub, 2013; Silfver, 2007). Kitayama, Markus, and Matsumoto (1995) suggest that the link between shame and defensive reactions, such as anger, is distinctive of individualist cultures where the sense of the independent self is highly appreciated, and where shame can thus be perceived as a sign of weakness. From this perspective, hiding shame with anger is reasonable in individualist cultures, but unreasonable in collectivist cultures. This is because in collectivist cultures where interdependence is highly valued, defending the self against shame in this way would likely be seen as inadequate. Demonstrating shame to others is seen as better, because it helps to maintain relationships and social cohesion. In fact, Bagozzi et al. (2003), comparing Dutch and Filipino salespeople's experiences of shame as a result of customer actions, found that they have similar experiences of shame but the behavioural consequences of emotion are different. For Filipino salespeople, shame improved customer relationship-building, whereas for Dutch salespeople, shame diminished it. In other words, behavioural intentions with the shame arousal appear to be highly related to cultural contexts. Thus, the following hypothesis is proposed:

H11: The impact of shame arousal on message compliance is moderated by personal cultural orientation. Specifically, (a) individualist individuals experiencing shame are less likely to comply with the message, whereas (b) collectivist individuals experiencing shame are more likely to do so.

\subsubsection{Guilt versus Shame}

As mentioned previously, the dominant models of shame and guilt claim that guilt leads to reparative action while shame does not, regardless of culture (Wong \& Tsai, 2007). Specifically, research aligns guilt with subsequent approach behaviours that maintain 
existing relationships and shame with avoidance behaviours that protect the self at the expense of social bonding (Tangney et al., 2007).

Analogous patterns have been observed in several studies concerning the links between shame, guilt, and empathy. It has been shown that proneness to shame is connected with a lower level of empathy, whereas proneness to guilt correlates positively with empathy (Tangney, 1991, 1995). It has also been observed that shame is negatively, and guilt positively, connected with psychological well-being (Orth, Robins, \& Soto, 2010). This shows a strong relationship between proneness to shame with a tendency to externalise blame, anger, and aggression (Dean \& Fles, 2016; Stuewig et al., 2010). There is, thus, a lot of empirical evidence that confirms the strong relationship between shame and the occurrence of unfavourable social behaviour, maladaptation, and psychological disorders. Besides, shame-free guilt is found likely to be adaptive and the presence of shame makes pro-social behaviour less likely (Bennett, 1998; Boudewyns et al., 2013). In sum, guilt rises empathy and the desire to apologise and make amends, while shame attempts to escape from social attention and one's own awareness of the transgression (Tangney \& Fischer, 1995; June Price Tangney et al., 1996). It is evident that guilt is, generally, a more adaptive emotion than shame.

As noted, in this study, emotional arousals are examined rather than emotional appeals. Formally, Hypothesis 12 states the following.

H12: Guilt arousal is more likely than shame arousal to lead to message compliance.

\subsection{Control Variables}

Several variables are used as control variables for their influence on health message persuasiveness.

\subsubsection{Health Message Exposure}

As previously mentioned in Section 2.3.3. Persuasion Knowledge Model, consumers' persuasion knowledge is positively related to advertising exposure (Mangleburg \& Bristol, 1998; Schaefer et al., 2005). Thus, exposure to health messages is a strong predictor of message persuasiveness (e.g., message compliance). 


\subsubsection{Message Involvement}

Message involvement is known as a state of focusing on and paying careful attention to a message (Andrews, Durvasula, \& Akhter, 1990). Message involvement has been found to be associated with stronger responses to persuasive appeals (e.g., Roser, 1990). Consequently, involvement in a message will influence how individuals attend and respond to a health message.

\subsubsection{Health Value}

Health value is defined as the importance of health to an individual (Lau, Hartman, \& Ware, 1986). Previous studies have revealed that health value is positively related to attitudes towards health protective behaviours (Moorman, 1994; Smith, Wallston, \& Smith, 1995) and thus health value positively influences the compliance towards health messages.

\subsection{Conceptual Model}

The conceptual model for research is proposed (see Figure 3.1).

\subsection{Chapter Summary}

This chapter presented the conceptual model and hypotheses of this study. The major relationships between constructs and the relevant hypotheses were discussed based on previous literature. The 12 hypotheses discussed in this chapter form the basis of the present study. The next chapter will discuss the research methodology used and approach undertaken in order to answer the research questions and test these hypotheses. 


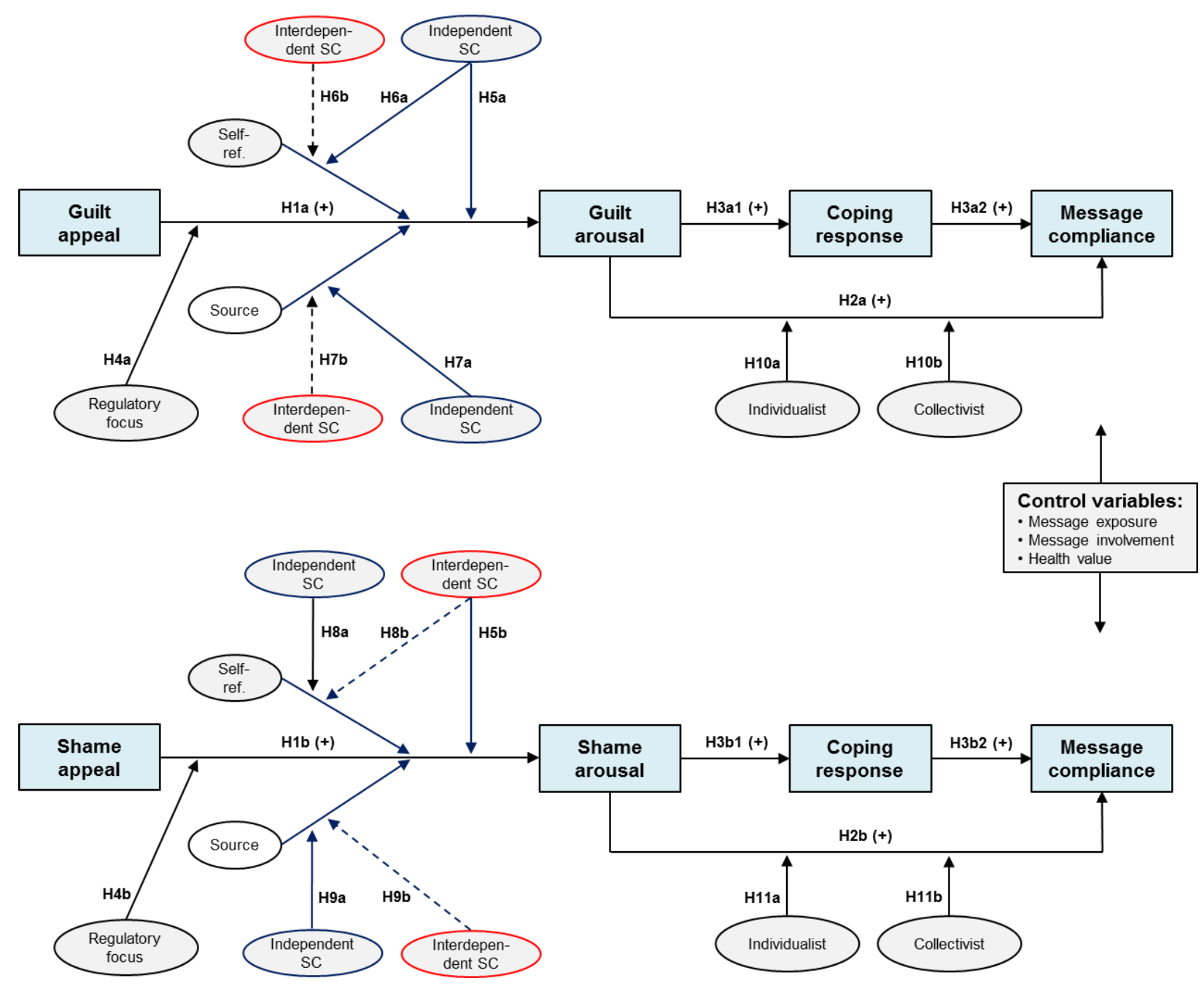

Note: SC: self-construal, ref.: referencing; (+): positive relationship; - $\rightarrow$ : no impact.

Figure 3.1. Conceptual Model of the Effectiveness of Guilt and Shame Appeals on Health Communications 


\section{Chapter 4. Research Methodology and Method}

\subsection{Introduction}

This chapter covers the research methodology and data collection procedures adopted to test the conceptual model and hypotheses developed in Chapter 3. The chapter begins with research paradigm and methodology. The experiment design and survey methods are accordingly discussed. The procedures employed for sample selection and data collection is detailed next, followed by a description of the analytical techniques used [involving structural equation modelling analysis and analysis of covariance] for testing the hypotheses and answering the research questions.

Figure 4.1 outlines the chapter.

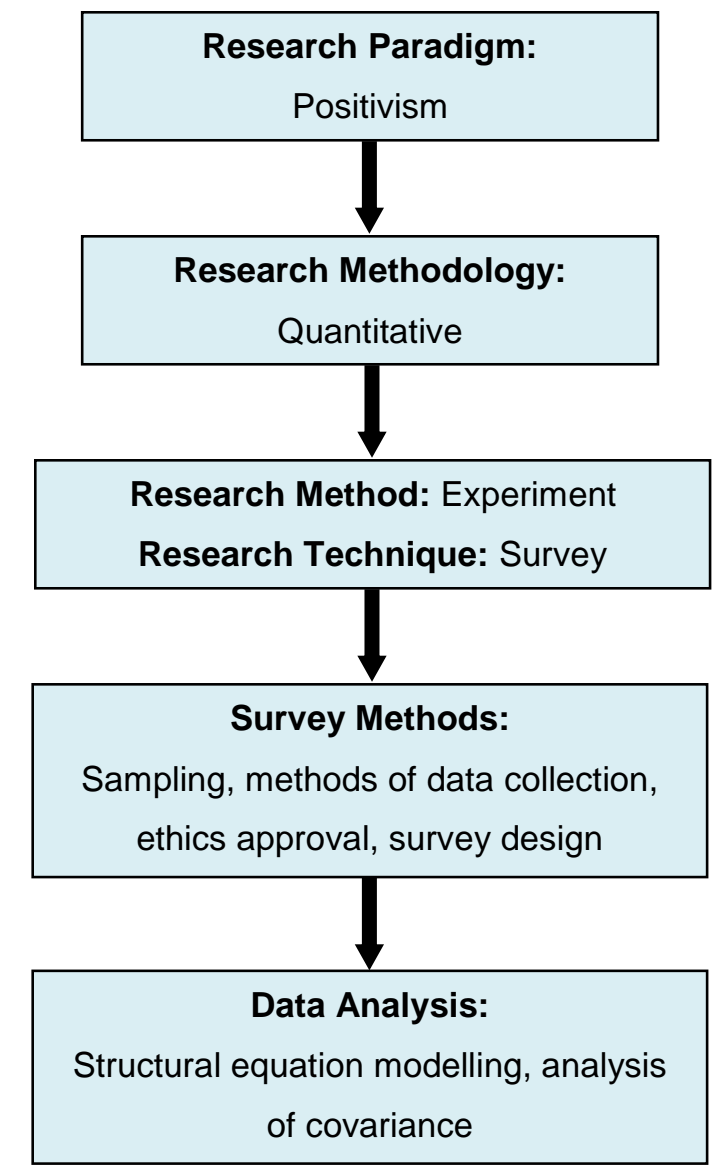

Figure 4.1. Outline of Chapter 4 


\subsection{Methodology}

\subsubsection{Research Paradigm}

A paradigm may be described as the underlying assumptions and intellectual structure on which research and development in a field of inquiry is based (Kuhn, 2012). That is to say, there are different ways in which researchers may interpret frameworks or view the world. These paradigms may be categorised by "the way their proponents respond to three basic questions, which can be characterised as the ontological [what is the nature of 'reality'?], the epistemological [what is the relationship between the inquirer and the knowable?], and the methodological [how should the inquirer go about finding out knowledge?] questions" (Guba \& Lincoln, 1994, p. 18).

Ontologically, researchers need to ask about the way things that exist are understood and categorised (O'Leary, 2013). Through the eyes of positivists, reality exists and its nature is there to be discovered (Guba, 1990). On the other hand, interpretivists believe that although reality does exist, its laws cannot be fully understood because reality is constructed based on humans' subjective experiences and perceptions (Guba, 1990). In exploring the phenomenon pertaining to the relationship between guilt/shame appeal and behavioural change, the present study reflects the belief that reality exists but it can only be approximated, due to the subjective nature of individuals' perceptions of selfconscious emotion (Creswell, 2009). Despite this, reality may be revealed through research (Guba, 1990) so this study has taken a positivist view.

In observing the relationship between the researcher and respondents of the research (epistemology), the present study reflects the belief that objectivity is relevant. Nevertheless, it is understood that objectivity cannot be achieved in any complete or absolute sense; instead, it can only be achieved closely by striving to be as neutral as possible (Guba, 1990). This epistemological view of the present study is, again, of the positivism paradigm. That is, the adoption of a distant and non-interactive position between the outcomes of inquiries and the interests and values of the inquirer (Guba, 1990; Hanson \& Grimmer, 2007).

Lastly, the methodological question asks how the inquirer should go about finding knowledge (Guba, 1990). The answer that is given to this question may be constrained by the answers already given to the ontology and epistemology questions (Guba \& Lincoln, 
1994). From both the ontological and epistemological categories, the present study reflects the view that objectivity is an important factor in conducting its research; however, it cannot be achieved in its absolute form (Creswell, 2009). Consequently, the present study selected methodologies that are intended to overcome this shortcoming, which allow the generation of findings on human behaviour that are generalisable for universal knowledge (Stiles, 2003). This enables the subjectivity of a particular respondent and researchers' bias to be minimised. Therefore this study adopted the positivism perspective in its methodology for studying the phenomenon of self-conscious emotion and its effects on individuals' behaviour (Guba, 1990).

Based on the discussion above, the present study took the positivism paradigm stance in conducting its research.

\subsubsection{Research Methodology}

Research that adopts the positivist perspective typically uses quantitative methods of data gathering and statistical analysis to confirm or disconfirm the hypotheses under review (Creswell, 2009; Sale, Lohfeld, \& Brazil, 2002).

Quantitative research tests theories through the examination of relationships among variables (Creswell, 2009). Under quantitative methods, 'good' research may be judged based on "the objective observation, precise measurements, statistical analysis and verifiable truths" (Cavana, Delahaye, \& Sekaran, 2001, p. 34). Cavana et al. (2001) noted that quantitative methods allow the measure of the phenomenon under investigation to be as nearly independent of the researcher's subjectivity as possible. This independence is paramount in discovering the true knowledge of said phenomenon and in generalising the result from the sampled test to the population of interest (Aaker, Kumar, Day, Lawley, \& Stewart, 2007; Cavana et al., 2001). It follows that quantitative research represents the positivist view.

According to Creswell (2009), the research methods being used should be consistent with the way research questions, objectives and hypotheses are phrased. Quantitative research methods are suitable when the question asks 'Do' or 'Does' instead of the 'How' or 'What' that is associated with qualitative research (Creswell, 2009). It was appropriate for the present study to use quantitative methods as it asked (i) "Do self-construal, regulatory focus and personal cultural orientation moderate the relative effectiveness of guilt and 
shame appeals towards health message compliance?"; (ii) "Does the coping response to guilt and shame arousals influence health message compliance?" A research question with a quantitative nature is useful in gaining a deeper understanding about the relationships among variables that the researcher seeks to understand (Creswell, 2009). A current research question compared the independent and dependent variables, namely emotional appeal and message compliance variables respectively. Quantitative research is also useful in testing well-known theories (Creswell, 2009). The theoretical foundations of the variables studied (such as emotion arousal, coping, and compliance) are established within various psychology and marketing contexts. This provides enough information to test these theories and generate hypotheses in the new context of health communications. Based on the above discussion on quantitative research methods, it was deemed appropriate to use them as the main methodology adopted here.

There are several methods of inquiry within quantitative research, including surveys and experiments (Cavana et al., 2001; Creswell, 2009). A survey can be designed to capture a range of information on many diverse topics and subjects of interest to the researchers (Aaker et al., 2007; Creswell, 2009). Therefore, considering the high number of constructs in the conceptual framework, and the incorporation of some adapted measures, this study took a survey research approach. Another reason for using this approach suggested by Aaker et al. (2007), is that it is best for evaluating relationships between variables (e.g., emotional appeal, arousal, coping, and compliance with emotion). The appropriateness of survey research for the present study was also due to its ability in transforming collected data into statistical values that could then be analysed to aid in the acceptance or rejection of the proposed hypotheses (Cavana et al., 2001; Creswell, 2009).

An experiment is a specific research method in which conditions are controlled so that the independent variable(s) can be manipulated to test a hypothesis about the dependent variable (Zikmund, Ward, Winzar, Lowe, \& Babin, 2014). As the primary focus of this study is to assess causal relationships between guilt and shame appeals and the emotional arousals, an experimental design is also adopted and incorporated into the survey. In the current case, an experiment is appropriate because the proposed independent variable, the emotional appeal, can be manipulated while controlling for extraneous variables that might have an impact on the emotional arousal being measured to enhance internal validity given time and budget (Hair, Bush, \& Ortinau, 2009). 


\subsubsection{Experiment}

\subsubsection{Experiment design}

Given that this study examines guilt and shame appeals in addition to self-referencing types and evaluation source types along with a control group, a 2 × 2 × 2 betweensubjects experimental design was employed. It consists of three manipulated variables: emotional appeal (two levels: guilt and shame), self-referencing (two levels: selfreferenced and other-referenced), and evaluation source (two levels: internal and external) (see Table 4.1).

Table 4.1. Experiment Design: 2 × 2 x 2 between-Subjects

\begin{tabular}{|c|c|c|c|c|}
\hline \multirow{2}{*}{ Emotion type } & \multicolumn{2}{|c|}{ Self-referencing } & \multicolumn{2}{|c|}{ Evaluation source } \\
\hline & Self-referenced & Other-referenced & Internal & External \\
\hline \multicolumn{5}{|l|}{ Guilt } \\
\hline Shame & & & & \\
\hline
\end{tabular}

Accordingly, the main experiment consists of eight treatment groups with a control group. The treatment group was randomly exposed to one of eight experimental treatments (i.e., self-referenced guilt, other-referenced guilt, internal guilt, external guilt, self-referenced shame, other-referenced shame, internal shame, and external shame appeal), while the control group was not exposed to any treatment/appeal.

\subsubsection{Choice of health issue}

Binge drinking is chosen as the health issue for this study on the basis of the following reasons. Firstly, alcohol is the most commonly used substance at all ages, especially adolescents and young adults (e.g., Cecilia \& Delyse, 2008). According to the World Health Organization (2015), 3.2\% of the burden of disease around the world is attributable to the consumption of alcohol, including death or disability. This made alcohol the third leading cause of burden of disease worldwide, especially alcohol consumption which causes death and disability relatively early in life. It is reported that out of all deaths among young people aged 15-29 years, 9\% is related to alcohol (World Health Organization, 2015).

Secondly, alcohol consumption is associated with a broad range of physical and social problems, at both personal and societal level (Rehm et al., 2003; World Health 
Organization, 2002). Personally, alcohol-related harm is pervasive, especially among young people (Cashell-Smith, Connor, \& Kypri, 2007; Conner, Langley, Tomaszewski, \& Conwell, 2003; McGee \& Kypri, 2004). Specifically, after drinking they often experience blackouts, unintended/unprotected sexual activity, academic impairment, short and longterm physical illness, and poor mental health, as well as antisocial risk behaviour, fights, and interpersonal consequences. The social-interpersonal harm includes depression, reduced self-esteem, and damaged relationships (Read, Kahler, Strong, \& Colder, 2006). Excessive alcohol consumption harms not only the drinkers, but also those surrounding them, including family, community, and society who suffer from crime, violence, and driving accidents (Hilbink, Voerman, van Beurden, Penninx, \& Laurant, 2012; Rehm et al., 2009; World Health Organization, 2002).

Thirdly, harmful drinking, such as binge drinking ${ }^{1}$, has increased among adolescents and young adults (World Health Organization, 2007). Particularly, harmful drinking is popular among university students (Kypri et al., 2009; Read et al., 2006). For example, in New Zealand, a national web-based survey reported that a total of $81 \%$ of both female and male university students drank in the previous four weeks, $37 \%$ reported one or more binge episodes in the previous week, and $68 \%$ scored in the hazardous range $(4+)$ on the AUDIT consumption subscale ${ }^{2}$ (Kypri et al., 2009). Similarly, studies in the U.S.A. show that approximately $44 \%$ of undergraduate students report at least one episode of binge drinking in the two weeks preceding the survey (Wechsler et al., 2002). Indeed, in countries where the comparison has been made, the prevalence of hazardous drinking among students has been found to exceed that of other young adults in the same age group (Dawson, Grant, Stinson, \& Chou, 2004; Kypri, Cronin, \& Wright, 2005; Windle, 2003). Heavy alcohol consumption among youths remains a public health concern in several countries (Karam, Kypri, \& Salamoun, 2007). It is because heavy drinking in young people leads to future alcohol dependence and alcohol-related injury (World Health Organization, 2001).

Finally, but most important, alcohol consumption is a significant public health concern and young binge drinkers are the focus of recent health promotion campaigns (e.g.,

\footnotetext{
${ }^{1}$ Generally, defined as consuming six or more standard drinks for men, five or more standard drinks for women, on a single occasion (Kypri et al., 2009).

${ }^{2}$ See the scale in Section 4.3.5.6.
} 
Jefferis, Power, \& Manor, 2005). However, it is evident that knowledge of the health risks for binge drinking provided by the campaigns has not translated into a reduction in binge drinking behaviour (Johnston \& White, 2003; O'Malley, Johnston, \& Bachman, 1998). As a consequence, substantial efforts to find a comprehensive solution for the abovementioned health issue in young adults, especially students, should be continuously undertaken and the importance of health communications increasingly emphasised. Although the chosen context and goal is preventing binge drinking, it is noted that the knowledge gained could be generalised to other health contexts.

\subsubsection{Experiment development}

The stimulus print materials used in the study are manipulations of guilt and shame appeals with self-referencing types and evaluation source types. The stimulus print material paradigm has previously been used in guilt and/or shame appeal research in the health contexts (e.g., Agrawal \& Duhachek, 2010; Boudewyns et al., 2013; Duhachek et al., 2012). In practice, print materials are widely used in health promotion (Bull, Holt, Kreuter, Clark, \& Scharff, 2001; Grol \& Grimshaw, 2003) for several reasons. First, they are a traditional way of disseminating information and are well understood by adult populations (Giguère et al., 2012; Straus, Tetroe, \& Graham, 2011; Vernon, McQueen, Tiro, \& Del Junco, 2010). Second, in comparison to other media, print ads have shown similar if not better effectiveness in terms of impacting primary outcomes such as behaviour and knowledge change, and are more cost-effective (Wilson et al., 2012; Wilson et al., 2010). The appropriateness of a print ad for the stimuli is also due to its flexibility required to substantiate the necessary information manipulations, while remaining high on ecological validity in which the print ad is typical to what people might normally be exposed to in health communications.

In this study, guilt and shame appeal ads are newly developed. They are guided by previous guilt and/or shame appeal research in health issues, such as binge drinking (Agrawal \& Duhachek, 2010; Duhachek et al., 2012) and screening for sexually transmitted diseases (Boudewyns et al., 2013), the literature of guilt and shame (e.g., Tangney \& Dearing, 2002), and considered the most prevalent alcohol-related harm/consequences the students/youth adults experienced (Read et al., 2006). As previously noted, anticipatory guilt/shame is chosen for this study, thus anticipated consequences of harmful drinking prevention rather than alcohol use disorder treatment, 
per se, is the health issue of choice. However, it is worth noting that, guilt and shame in this study are aroused from generalised guilt- and shame-eliciting tactics rather than contextual alcohol use disorder-related tactics (see Guilt and Shame arousal measure in Section 4.3.5.2).

Tangney and Dearing (2002) suggested, for engineering a guilt appeal, emphasising the behaviour (e.g., irresponsible drinking behaviour) rather than the self, involving the consequences for others (e.g., others could suffer), and guiding the development of reparative skills (efficacy). For a shame appeal, Boudewyns et al. (2013) suggested focusing on the self (e.g., irresponsible drinker/s), de-emphasising the consequences for others (empathy) (e.g., others could observe), and cutting off the efficacy cue. In terms of efficacy in guilt and shame appeals, Duhachek et al. (2012) proposed that gain frames would have more efficacy (e.g., if you drink responsibly...), whereas loss frames would have less (e.g., if you drink irresponsibly...). This is because gain frames representing challenge appraisals emphasise perceptions of opportunity and promote a high confidence in one's ability to cope; while loss frames representing threat appraisals reflect a perception of potential danger, and promote a low confidence in one's ability to cope (Lazarus, 1991; Skinner \& Brewer, 2002). Furthermore, in a study of the effectiveness of anti-drinking messages, Agrawal and Duhachek (2010) suggested that ad frames accentuating the role of others as observers of the negative consequences of a transgressor should induce shame and ad frames accentuating the role of others as sufferers of the negative consequences of a transgressor should induce guilt for that person.

Table 4.2. Emotional Type's Highlights and Consequences of Drinking

\begin{tabular}{llll}
\hline Emotion type & Guilt & Shame & Sources \\
\hline Highlights & The behaviour & The self & Agrawal and Duhachek \\
& Consequences for others & De-emphasising the & (2010); Boudewyns et al. \\
& (others as sufferers) & $\begin{array}{l}\text { consequences for others } \\
\text { (2013); Tangney and }\end{array}$ & $\begin{array}{l}\text { Dearing (2002); } \\
\end{array}$ \\
& Reparative skills/efficacy & Cutting-off the efficacy & Duhachek et al. (2012) \\
& cue (gain frame) & cue (loss frame) & \\
\hline Consequences & Blackout, risk behaviour, social-interpersonal & Read et al. (2006) \\
\hline
\end{tabular}


In terms of consequences, the stimulus ads highlight the most significant alcohol-related harm the students/youth adults experienced. These consequences include blackouts, risk behaviours, and social-interpersonal consequences (Read et al., 2006). See Table 4.2 for highlights of the guilt and shame appeals and significant consequences of drinking.

For the particular health issue of binge drinking prevention, the stimulus ads targeted health behaviour: harmful drinking, with the key message: "Drink responsibly" (see Table 4.3). For emotional appeal manipulation, Dillard and Nabi (2006) suggest that it begins with identifying the emotion to be aroused (guilt and shame), and then creating a message based on the corresponding appraisal pattern (see Table 4.2): the behaviour focus and the self focus, respectively (Lewis, 1971; Tangney \& Dearing, 2002). Two template appeal ads were created to stimulate anticipatory guilt and shame. Accordingly, the anticipatory guilt appeal focuses on the behaviour of a potentially committed actor, e.g., "Binge drinking" and the shame appeal focuses on the self as a potentially committed actor, e.g., "Irresponsible drinker". Then, each template ad incorporates self-referencing types or evaluation source types. For the self-referencing, adopted from Block's (2005) study on self-referenced guilt appeals, self-referenced guilt/shame appeal references the receiver's behaviour/self, e.g., "You are a transgressor", other-referenced guilt/shame appeal references the others' behaviour/self, e.g., "Others are transgressors". For the evaluation source, internal guilt/shame appeal mentions the actor's decision will be involved in or known/seen by him/herself, e.g., "You are a sufferer/an observer", external guilt/shame appeal mentions the actor's decision will be involved in or known/seen by the others, e.g., "Others are sufferers/observers". The manipulation of evaluation source is consistent with previous studies on guilt- and shame-focused attitudes (Baumeister et al., 1994; Gilbert et al., 2007). See Table 4.3 for key points of the text of stimulus ads for binge drinking prevention.

For the ad design, adopted from Paul et al.'s (1997) checklist of content and design characteristics for effective print health promotion materials, the stimulus ads had the characteristics incorporating both content and design. These include full colour with large clear font, structured paragraphs with repeated important points, and illustrations. In previous experimental studies, guilt and/or shame appeal manipulations have incorporated both text and illustration (e.g., Agrawal \& Duhachek, 2010; Boudewyns et al., 2013; Duhachek et al., 2012). 
Table 4.3. Health Issue and Stimulus Ads' Key Points

\begin{tabular}{lll}
\hline Health issue & Target behaviour & Key message \\
\hline $\begin{array}{l}\text { Binge drinking } \\
\text { prevention }\end{array}$ & Harmful drinking & Drink responsibly \\
\hline Appeal type & Guilt & Shame \\
\hline Self-referenced & YOU binge drink; & YOU are an irresponsible drinker; \\
& You are a transgressor & You are a transgressor \\
Other-referenced & OTHERS binge drink; & OTHERS are irresponsible drinkers; \\
& They are transgressors & They are transgressors \\
Internal source & You binge drink; & You are an irresponsible drinker; \\
& YOU are a sufferer & YOU are an observer \\
External source & You binge drink; & You are an irresponsible drinker; \\
& OTHERS are sufferers & OTHERS are observers \\
\hline
\end{tabular}

Eight stimulus print ads were developed for two guilt and shame appeals, each differing in either self-referenced or other-referenced, and either internal or external evaluation sources. They were identical in content, design characteristics with the exception of the manipulation section to avoid confounding effects. All stimulus ads were structured similarly, composed of two parts: the text (e.g., headline, body, and tagline) and the illustration (e.g., image). For a detail of the ads' text and image, see Appendix 1. The headline highlights the expected emotional arousal, guilt and shame respectively. The body serves as the manipulation section (e.g., type of emotional appeal, type of selfreferencing, type of evaluation source). The tagline or key message always highlights the target behaviour. The image portrays a person vomiting in a toilet bowl, modified from Agrawal and Duhachek's (2010) and Duhachek et al.'s (2012) studies to support the key message. In the current study, two images were used in which male participants were exposed to a male image and females the female image.

In all, the body section used in the stimulus ads provided the treatment conditions for this study's experiment. It should be noted that the present study differentiated guilt and shame by varying only whether the stimulus ad focused on the behaviour (guilt) or the self (shame) so as to provide a more carefully controlled comparison. This also helps rectify the manipulation issue caused by many previous studies employing different topics, different illustrations, and different guilt- or shame-eliciting message tactics for 
both within and between the guilt and shame appeals (Boudewyns et al., 2013). See Section 4.2.3.4 below for the results of pretesting these stimulus ads.

\subsubsection{Stimulus pretesting}

Prior to the experiment, stimulus pretesting was conducted on university students who did not take part in the main experiment. The pretest occurred between the months of November and December 2015. During the test, the link to the pretest survey was distributed to several 200 and 300 level courses at Victoria University of Wellington through the University's Blackboard site. Participants randomly received one of eight stimulus ads regarding binge drinking (see Appendix 1).

Five pretests were checked to ensure that the stimulus worked effectively. The stimulus consisted of three manipulations: 2 emotional appeal (guilt versus shame) x 2 selfreferencing (self-reference versus other-reference) x 2 evaluation source (internal source versus external source). The two gender-specific images used and the intensity level of the emotional appeals were also pretested.

Table 4.4. Distribution of Manipulations

\begin{tabular}{lcc}
\hline Manipulation & $\mathbf{N}$ & $\%$ \\
\hline Self-referenced guilt & 34 & 13.0 \\
Other-referenced guilt & 32 & 12.3 \\
Internal guilt & 35 & 13.4 \\
External guilt & 28 & 10.7 \\
Self-referenced shame & 31 & 11.9 \\
Other-referenced shame & 33 & 12.6 \\
Internal shame & 35 & 13.4 \\
External shame & 33 & 12.6 \\
Total & $\mathbf{2 6 1}$ & $\mathbf{1 0 0 . 0}$ \\
\hline
\end{tabular}

There were 261 respondents who completed the pretest: 82 males (31\%) and 179 females (69\%). There was a nearly equal distribution of stimulus manipulations (see Table 4.4).

\section{Images used}

This pretest assessed two images used in the stimulus ads in order to determine if they were visually appropriate. After viewing a manipulated ad, participants were asked to 
respond: (1) attention to image: "To what extent does the image attract your attention?" on a 7-point scale ranging from $1=$ 'not at all' to 7 = 'extremely' (Boudewyns et al., 2013); (2) realism of image: "Is the image realistic?"; and (3) image relevance: (i) "The image made me think of my personal experiences in similar scenarios"; (ii) "The image seemed to relate to myself"; (iii) "The image seemed to relate to people who are close to $m e$ "; and (iv) "I can easily picture myself in the situation portrayed in the image" anchored by $1=$ 'strongly disagree' to $7=$ 'strongly agree' (Cronbach's $\alpha=.85$, Martin et al., 2013).

\section{Table 4.5. Image Check}

\begin{tabular}{lccc}
\hline & $\mathbf{N}$ & Mean & SD \\
\hline Attention to image & 261 & 3.89 & 1.630 \\
Image relevance & 261 & 3.22 & 1.550 \\
Male & 82 & 3.44 & 1.472 \\
Female & 179 & 3.12 & 1.579 \\
\hline Realism of image & $\mathbf{N}$ & $\%$ & \\
\hline Yes & 190 & 72.8 & \\
No & 71 & 27.2 & \\
Total & $\mathbf{2 6 1}$ & $\mathbf{1 0 0 . 0}$ & \\
\hline
\end{tabular}

The images were selected appropriately for the following reasons (see Table 4.5): (1) overall attention to image rating was moderate $(M=3.89) ;(2)$ the majority of respondents had agreed with the realism of image on the second measure with $n=190,72.8 \%$; (3) overall rating to image relevance was moderate $(M=3.22)$. Furthermore, the difference between males and females with regards to the respective image relevance was not significant, $t(259)=1.548, p=.123$.

\section{Emotional appeal type}

In order to test the emotional appeal type in which guilt focused on the behaviour and shame focused on the self, after viewing a manipulated ad, participants were asked to rate: "According to the advertisement, what was the focus of binge drinking?" on a 7point scale ranging from $1=$ 'the behaviour' to $7=$ 'the self' (Lewis, 1971; Tangney \& Dearing, 2002). 
The difference in ratings on the focus between guilt and shame appeal type was significant, $t(259)=-2.045, p=.042$. Results show that the mean score of the behaviour $(M=3.65)$ is significantly lower than the self $(M=4.18)$. This suggests that the guilt and shame appeal type manipulation is effective.

\section{Self-referencing}

To test the type of self-referencing, participants viewed a manipulated ad, then rated: "According to the advertisement, rate the extent to which binge drinking could be committed by..." on a 7-point scale ranging $1=$ 'yourself' to 7 = 'others' (Block, 2005).

There was a significant difference in ratings on self-referencing between self-reference and other-reference, $t(128)=-2.134, p=.035$. Results show that the mean score of yourself $(M=3.25)$ is significantly lower than others $(M=3.94)$. This suggests that the self-referencing manipulation is effective.

\section{Sources of evaluation}

In order to check the type of evaluation source, after viewing a manipulated ad, participants were asked to rate: "According to the advertisement, rate the extent to which the consequences of binge drinking could be known/seen by..." on a 7-point scale ranging from 1 = 'yourself' to 7 = 'others' (Brommersma, 2007; Gilbert et al., 2007; Kim et al., 2011).

The difference in ratings on source of evaluation between internal and external was significant, $t(129)=-5.230, p=.000$. This suggests that the evaluation source manipulation is effective as the mean score of yourself $(M=3.73)$ is significantly lower than others $(M=5.26)$.

\section{Intensity level of emotional appeal}

The final pretest checked the intensity level of emotional appeal to ensure that guilt appeal elicits significantly more felt emotion of guilt than shame and shame appeal significantly more felt emotion of shame than guilt. Participants each viewed a manipulated ad and were then asked to rate: (i) "How successful was the advertisement in attempting to make the viewer feel guilt?"; and (ii) "How successful was the advertisement in attempting to make the viewer feel shame?" anchored by 1 = 'not at all' to 7 = 'extremely' (Coulter \& Pinto, 1995). 


\section{Guilt}

The differences across four types of guilt appeal in terms of felt guilt were not significant, $F(3,125)=2.599, p=.055$. Results show that the mean score of felt guilt was moderate $\left(M_{\mathrm{G}}=3.48\right)$. Besides guilt, guilt appeal also elicited shame but with a lower mean score $\left(M_{\mathrm{S}}=3.24\right)$. As predicted, guilt appeal resulted in significantly more guilt than shame, $t(127)=2.824, p=.005$.

Furthermore, it was expected that across gender they would not significantly differ in terms of felt guilt. Results reveal that the difference between males and females was not significant, $t(127)=-1.669, p=.098$.

\section{Shame}

The difference across four types of shame appeal in terms of felt shame were also not significant, $F(3,128)=.471, p=.703$; the mean score of felt shame was moderate $\left(M_{\mathrm{S}}=\right.$ 3.83). Besides shame, shame appeal also evoked guilt but with a lower mean score $\left(M_{\mathrm{G}}=\right.$ 3.67). As expected, shame appeal resulted in significantly more shame than guilt, $t(130)=$ $2.162, p=.032$.

In addition, it was expected that across gender they would not significantly differ in terms of felt shame. Results also reveal that the difference between males and females was not significant, $t(130)=-1.617, p=.108$.

Thus, the manipulated ads pretested with both male and female students in New Zealand were effective.

\subsection{Survey Methods}

\subsubsection{Sampling}

\subsubsection{Sample frame}

The sample framing used in this study follows previous studies involving drinking messages (e.g., Agrawal \& Duhachek, 2010; Duhachek et al., 2012; Martin et al., 2013). The participants were undergraduate students from New Zealand universities. Undergraduate students were selected for the sample for several reasons. First, binge drinking is certainly pertinent to adolescents and young adults (World Health Organization, 2007); particularly as harmful drinking rates have increased most rapidly 
among university-aged students (Kypri et al., 2009). University students, besides drink more heavily than their peers (other young adults in the same age group), have a higher prevalence of alcohol use disorders (Slutske, 2005). These make university students a high-risk subgroup of the highest risk age group in the population.

Second, student samples have previously been used in many theory testing studies where multivariate relationships are examined (e.g., Kim, 2004). Third, students are wellmatched samples to each other. The sample comparability is required for such a betweensubjects experiment to avoid a confounding effect, in which the results are interrupted by something else other than individual differences such as education, social status, wealth, and age (Jilke, Petrovsky, Meuleman, \& James, 2016). Finally but importantly, as adhered to Victoria University of Wellington's Human Ethics Committee standards to protect the safety, health, and welfare of human subjects, students were chosen rather than broader population groups (e.g., older persons). Guilt and shame are unpleasant emotions and might be uncomfortable for participants to experience. In such cases, the University was able to provide students with referrals for counselling to the Student Counselling Service if they wished.

\subsubsection{Sampling method}

Non-probability, more specifically, convenience sampling was used partly due to the wide geographic distribution of the sample, and time and budget constraints.

As recommended for multivariate data analysis by Hair, Black, Babin, and Anderson (2010), a minimum size is 20 per cell (group) and sample sizes per group are approximately equal. Although the current study consisting of eight treatment groups with one control group would require 180 responses (9 groups x 20 per group) for an adequate analysis, the main study obtained a total of 301 responses (including the control group), thereby providing a very adequate sample size.

\subsubsection{Methods of Data Collection}

The survey was made available and responses were gathered online, using web-based survey software. There were several advantages in using web surveys to conduct the research. The Internet is able to provide access to unique populations (Hanna, Rohm, \& Crittenden, 2011). Undergraduate students, the study's target sample, are classified as the Net Generation and considered to be highly Internet-savvy (Hauben, 1997). Thus, the 
Internet is where the target sample was most accessible. A web survey also provided a time efficiency advantage by connecting with the targeted sample, even if dispersed geographically, in a timely manner (Garton, Haythornthwaite, \& Wellman, 1999; Hanna et al., 2011).

Web surveys also enabled the individuals to respond anonymously (Marquis, Marquis, \& Polich, 1986; van Hamersveld \& de Bont, 2007). Providing anonymity to the respondents was an advantage for the present study as respondents were more likely to answer the survey questions fully and in an unbiased manner based on their own perceptions of emotion arousal and the message compliance as opposed to what they considered appropriate answers. In addition, a web-based survey is self-administered, meaning that respondents were able to answer the survey at their convenience and researchers did not have to be present while respondents answered the survey questions. Therefore, respondents were more likely to provide thoughtful and unbiased responses (Evans \& Mathur, 2005; Wright, 2005). Lastly, web surveys are associated with low preparation and administration costs (Evans \& Mathur, 2005). In terms of administration costs, using a web survey allowed the present study to be cost effective by eliminating the need for paper, postage, interviewers and other costs commonly included in the traditional paper, mail, face-to-face or telephone formats (Evans \& Mathur, 2005; Hanna et al., 2011).

Nonetheless, there are some notable disadvantages in conducting surveys using webbased software. There is the possibility that the general public may perceive a web survey as unsolicited junk mail (also known as Spam), which may lower the response rate considerably (Evans \& Mathur, 2005). Data collection error is another disadvantage in using web-based surveys. Data collection error may arise from unclear instructions that may lead to misinterpretation (Evans \& Mathur, 2005) and respondents exiting the survey without finishing (Boulos \& Wheeler, 2007). In order to overcome these drawbacks, a pilot test was conducted, and instructions to the pretest and main study were made as clear as possible. The response rates to both tests suggested that these limitations had minimal effects on the present study.

Based on the above discussion, it was evident that using a web survey would have more advantages than drawbacks. A self-administered, anonymous web-based survey was used for both the pretest and main study. The surveys were created using Qualtrics online research software and were accessible to the respondents through a survey link. 
Distributing the survey link to the target sample for the main study occurred using several different approaches through Victoria University of Wellington's Blackboard site and social network sites (e.g., Facebook group pages).

It is well recognised that an incentivised survey is likely to increase the response rate of the survey (Poynter, 2001). Therefore, to encourage survey participation for the main study, an incentive in the form of a $\$ 100$ New World supermarket voucher was given to two winners randomly selected from the survey draw.

\subsubsection{Ethics Approval}

Prior to the web survey for the main study being activated, ethical approval from the Victoria University of Wellington's Human Ethics Committee (Ref: 21449) was obtained to ensure that research conducted under its auspices conformed to various statutory requirements (Victoria University of Wellington, 2016). Within the scope of this study, responses were anonymous and the survey adhered to the University's standards to protect the privacy, safety, health, cultural sensitivity and welfare of human subjects.

\subsubsection{Survey Design}

\subsubsection{Survey instrument}

The format of the survey instrument was highly important, as the researcher was not present to make adjustments or answer queries from the respondents. According to Cavana et al. (2001), when a survey is sent out, "it is at the mercy of the respondents whether they decide to complete it; whether they interpret the questions as intended; and whether they answer the questions honestly" (p. 226). As mentioned earlier, one of the disadvantages of web surveys is the possibility of data collection error due to unclear instructions (Boulos \& Wheeler, 2007; Evans \& Mathur, 2005). It was therefore vital that survey design dealt with these issues.

The survey was in an online format and was divided into several sections: (i) Information sheet, (ii) Definitions of binge drinking, (iii) Screening section, (iv) Stimulus presentation, ( $v$ ) Construct-related questions (main body), and ( $v i$ ) Concluding page. The information sheet presented the purpose of the study, instructions on how to answer the survey, information regarding ethics approval and anonymity responses, and a description of how the survey data would be used. The researcher and supervisors' contact details 
were also listed on the information sheet to ensure respondents' queries and issues regarding the survey and/or the study in general could be addressed. Next, definitions of binge drinking used throughout the survey were provided, followed by the screening questions (e.g., they are undergraduate students) used to determine the eligibility of the respondents in completing the survey. Based on gender male or female, a participant was randomly given an ad with a respective gender image.

The main body of the survey comprises four parts (see Appendix 2 for the order of variables). The first part includes three sections: manipulation checks (emotional appeal type and level, self-referencing type, evaluation source type), mediator measures (emotional arousal, coping response), and dependent measures (attitude towards the ad and the message, binge drinking intention, and message compliance). The second part consists of measures of control variables (health message exposure, message involvement, and health value) and explanatory variables (self-construal, regulatory focus, and personal cultural orientation). The third part of the questionnaire requests information on demographics: nationality, ethnicity, gender, and age. The final part measures the further dependent variable (actual behaviour). At the conclusion of the survey, respondents were thanked for their participation and were given a choice to end the survey or enter a draw to win a prize, in which case they were redirected to another secure site where they were able to provide their contact details. See Appendix 3 for the survey, laying out the format described above.

\subsubsection{Pilot-testing}

A pilot test was conducted on a group of 30 participants from New Zealand undergraduate students to test for the comprehension requirements of the stimulus and questionnaire, and to identify any problems encountered by the respondents (Zikmund et al., 2014). The number of participants selected was based on the suggestion that a pilot test sample size is generally in the range of 20 to 40 (Rea \& Parker, 2005).

All aspects of the questionnaire were tested, including scale items, question content, wording, form layout, question difficulty and instructions (Lietz, 2010). Some amendments were then made to the questionnaire based on the information provided from the pilot test. Notably, one item of independent self-construal sub-scale was eliminated due to a redundancy with one item of health value construct used as a control variable in the current study (see more in Section 4.3.5.1). 


\subsubsection{Measurements of Constructs}

The construct measurements for independent, dependent, control, and explanatory variables were generated on the basis of prior operationalisation and scales previously used in the literature adapted for this study context. All of these measurements, except for coping response which was measured by open-ended responses, used a 7-point scale. The rating scale was adopted owing to being a commonly used approach to scaling responses in survey-based research (Thompson, 1998). Given that 5-point and 7-point scales can easily be rescaled in order to facilitate comparisons (Gregory, 2008), the latter has been found to be more reliable than the former since a 7-point scale allows for a greater differentiation of responses, while not artificially increasing differentiation, as might be the case where more scale points are offered (Lietz, 2010).

The measures for the constructs are presented in Appendix 4, and are then modelled in Figure 4.2 Overall Measurement Model, including all independent/mediator, dependent and explanatory/moderator variables.

\subsubsection{Explanatory variables}

\section{Self-construal}

The Self-Construal Scale (Singelis, 1994) was used to assess independent and interdependent self-construals. The scale includes a 24-item self-report measure on a 7point Likert-type scale ( 1 = 'strongly disagree', 7 = 'strongly agree'). 12 items measure independent self-construal and the other 12 items measure interdependent self-construal. Prior research has established adequate construct validity, predictive validity, and internal reliability of this measure (Singelis, 1994), including studies using Asian (Kwan, Bond, \& Singelis, 1997) and Asian American (Cheung \& Park, 2010) college samples. Internal consistency estimates (Cronbach's $\alpha$ ) from a recent study (Park et al., 2011) were .76 and .81 for the independent and interdependent subscales, respectively.

In the current study, only 11 of the 12 sub-scale items were used in tapping independent self-construal. Item 12 "I value being in good health above everything" is redundant with an indicator of health value construct used as a control variable (item 4 "There is nothing more important than good health", see more in Section 4.3.5.5 below). Item 12 was subsequently deleted from the questionnaire. Consequently, the self-construal construct was measured by two sub-scales with 23 items. 


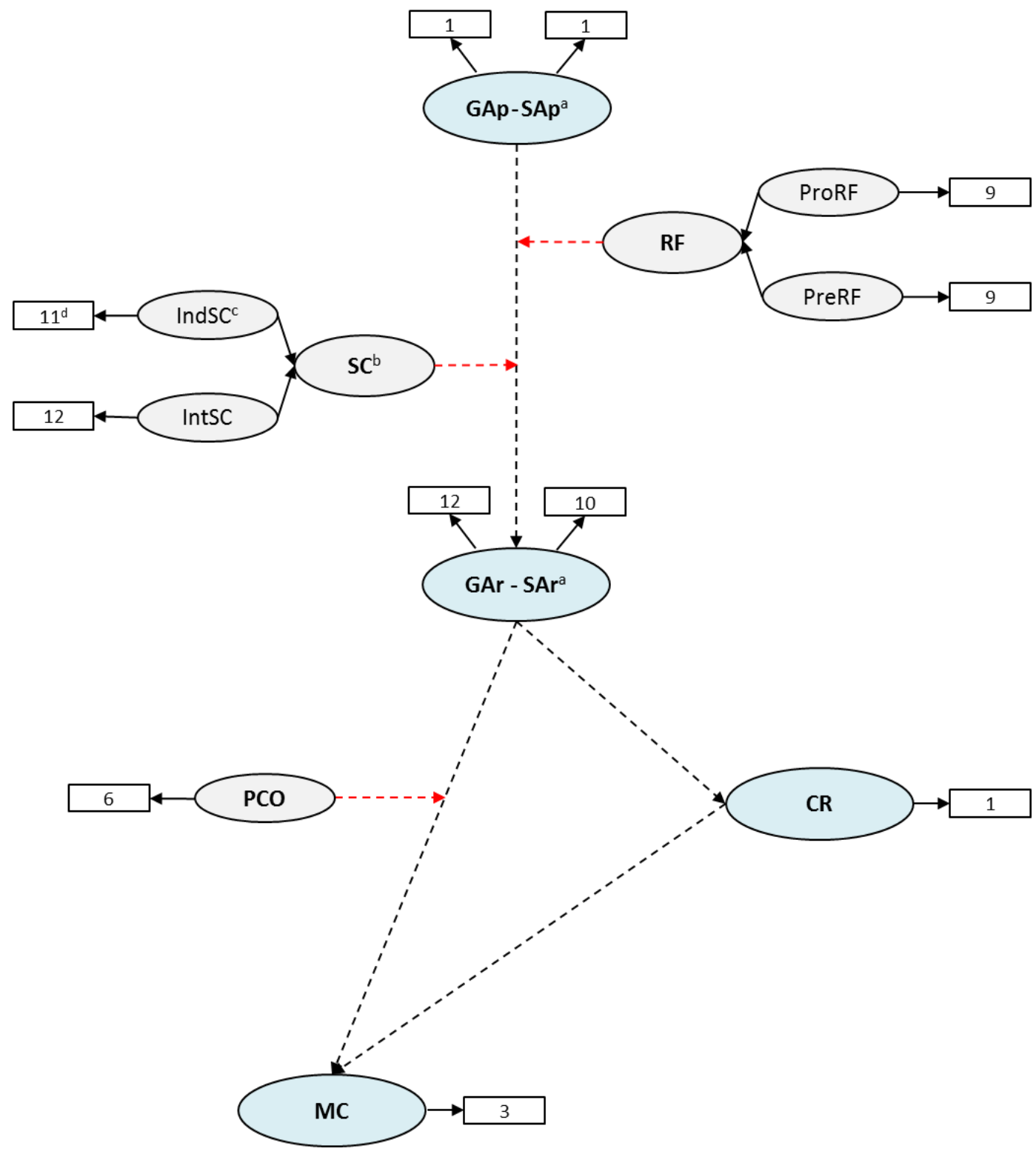

Note: $\longrightarrow$ : relationships between constructs and their measures, $\rightarrow \rightarrow$ : hypothesised relationships between

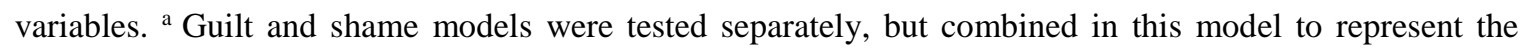
conceptual framework; ${ }^{\mathrm{b}}$ Latent variables; ${ }^{\mathrm{c}}$ Sub-latent variables; ${ }^{\mathrm{d}}$ Number of observed variables. See List of Abbreviations for explanations of acronyms.

\section{Figure 4.2. Overall Measurement Model}

\section{Regulatory focus}

The Regulatory Focus Scale (Lockwood et al., 2002) was used to measure the promotion and prevention focus of consumers. The scale has 18 items, half of which measure promotion focus and the other half measure prevention focus. Using a scale with endpoints of 1 ('not at all true') and 7 ('extremely true'), participants indicate the extent to which they endorsed items relevant to a promotion focus and items relevant to a 
prevention focus. Internal consistency estimates (Cronbach's $\alpha$ ) from a study (Zhao \& Pechmann, 2007) were .87 for promotion focus and .82 for prevention focus.

\section{Personal cultural orientation}

This study used the Personal Cultural Orientation Scale (Yoo \& Donthu, 2005) to measure personal cultural orientation. The scale was based on the Hofstede's (2001) work, but was applied and validated in a number of other studies (Schumann et al., 2010; Yoo, 2009; Yoo \& Donthu, 2002, 2005) measuring cultural orientation at the individual level: collectivism versus individualism. In a study, Yoo (2009) found $\alpha=.81$ for American sample and $\alpha=.79$ for Korean sample. A recent study (Schumann et al., 2010) conducted across 11 countries also validated the scale $(\alpha=.85)$ at the individual level.

The scale of personal collectivism-individualism includes six items measured with a 7point Likert-type scale of $1=$ 'strongly disagree' and $7=$ 'strongly agree'. Higher scores mean collectivist tendencies, lower scores mean individualist tendencies.

\subsubsection{Mediating variables}

\section{Guilt and Shame arousal}

The Adapted Shame and Guilt Scale (Hoblitzelle, 1987) was used to measure shame and guilt arousal. The scale is a generalised scale divorcing shame and guilt from the specific contexts in which they arise and make no reference to specific elements (e.g., phenomenological) of either emotion (Kim et al., 2011). Thus, this generalised scale is consistent with the nature of shame (e.g., global self) and guilt (e.g., specific behaviour) in this study rather than the contextual scale which is relevant to alcohol use disorderrelated shame and guilt.

The scale is an adjective-based measure that requires subjects to rate the extent to which shame or guilt-related adjectives describe the self. It includes 22 items on a 7-point scale in which 10 items measure the shame state and 12 items the guilt state. Hoblitzelle (1987) found the reliability coefficient Cronbach's $\alpha=.86$ for shame and .88 for guilt.

\section{Coping response}

The coping response measure was adapted from the Dominant Cognitive Response Scale (Dillard \& Peck, 2000). The scale was measured by coding open-ended responses, 
providing free-response descriptions of the thoughts and feelings subjects experienced in coping to an affective message, into three categories: supporting thoughts, neutral thoughts, and counter-arguments.

In this study, coping responses were then adaptively coded by two trained coders. A judge blind to respondents completed the coding where 1 = 'maladaptive coping' (e.g., counter arguments), 2 = 'neutral coping' (e.g., neutral responses), and 3 = 'adaptive coping' (e.g., supportive arguments). Higher scores indicate an adaptive coping response, conversely, lower scores indicate a maladaptive coping response. Another independent judge, also blind to participants' coping responses, completed the same coding. After coding all of the coping responses, the coders compared their codes and resolved their disagreements by discussion. The result showed the Kappa measure of agreement $(\kappa)$ value is .85 , with a significance of $p<.001$. According to Peat (2001, p. 228), $\kappa=.50$ represents moderate agreement, above .70 represents good agreement, and above .80 represents very good agreement. So, in this test the level of agreement between the classification of coping responses into three categories was very good.

Generally, information is represented by either numerical or categorical data. In the latter, variables take values in nominal scales (no comparison between possible categories), ordinal scales (where categories are completely ordered), and partially ordered scales (with a partial order in categories) (e.g., Torra et al., 2006). In the current study, coping response was totally ordered, thus it could be used as a continuous variable (Acock \& Martin, 1974; Harwell \& Gatti, 2001). It should be also noted that the coping response was measured by open-ended responses, then adaptively coded into the three codes in order to: $(i)$ explore as many of possible cognitive responses to the arousal of guilt or shame as possible to explain the mediating effect of coping response more fully; (ii) conduct path analyses to test that effect of coping response (see Section 5.6.1). Consistently with previous research on health messages (i.e., Reinhart, Marshall, Feeley, \& Tutzauer, $2007^{3}$ ), a measure of coping response was scored by taking the mean on these three codes.

\footnotetext{
${ }^{3}$ A study where thought-listing was used to measure reactions to the message framing manipulation. Each thought was coded for 'valence' or how positive the thought was towards either the message or organ and tissue donation: negative (coded as -1 ), neutral $(0)$, or positive $(+1)$. These codes were then summed for an aggregate measure of valence.
} 


\subsubsection{Dependent variable}

\section{Message compliance}

Message compliance was measured by a set of three statements with a 7-point Likert-type scale ( 1 = 'strongly disagree', 7 = 'strongly agree') adopted from Yu and Shen (2012) to evaluate the likelihood that subjects would take the actions that the messages advocated, with the reliability coefficient to be very good (Cronbach's $\alpha=.96$ ).

\subsubsection{Further dependent measures}

Further measures of the dependent variable include factors correlated with message compliance such as behaviour intent (as measured by Binge drinking intention and Actual behaviour) and attitude (Attitude towards the ad and Attitude towards the message) (Kühberger, 1998).

\section{Binge drinking intention}

Intention to binge drink was measured by a statement: "How likely are you to engage in binge drinking this year?" on a 7-point Likert scale $(1=$ "not at all likely", 7 = "very likely") (Agrawal \& Duhachek, 2010; Duhachek et al., 2012). Respondents were provided with the definition of binge drinking specific to New Zealanders (e.g., 6+ standard drinks for men and 4+ standard drinks for women on a single occasion, New Zealand Ministry of Health, 2015).

\section{Actual behaviour}

Actual behaviour was measured by two questions on actions that had been taken, adapted from Reinhart et al. (2007): (i) "Did you click on the hyperlink?"; (ii) "Did you look at the website that provided additional information regarding binge drinking?"

\section{Attitude towards the ad}

Attitude towards the ad was measured by three 7-point semantic differential items anchored with 'uninformative/informative', 'ineffective/effective', and 'not believable/believable' adapted from the Attitude towards the public service announcements scale in its drug use study, with Cronbach's $\alpha=.86$ (Schoenbachler \& Whittler, 1996). 


\section{Attitude towards the message}

Attitude towards the advocated message was measured by four 7-point semantic differential items: 'not appropriate/appropriate', 'harmful/beneficial', 'unrealistic/realistic', and 'unconvincing/convincing' adapted from Norman and Smith (1995) to measure attitude towards taking regular exercise, with Cronbach's $\alpha=.83$.

\subsubsection{Control variables}

In the current study, several confounding variables may affect compliance with health message, including health message exposure (Mangleburg \& Bristol, 1998; Schaefer et al., 2005), message involvement (Andrews et al., 1990; Roser, 1990), and health value (Moorman, 1994; Smith et al., 1995).

\section{Health message exposure}

The scale was adopted from Ridout, Shah, Goldstein, and Franz (2004) to measure the exposure to televised advertising by asking respondents how much attention they had paid to health communications in the last three months: a lot, some, very little, or none.

\section{Health message involvement}

The Personal Involvement Inventory Scale (Zaichkowsky, 1985) was adopted to measure involvement with general health messages. The scale has six 7-point bipolar semantic differential items anchored with 'unimportant/important to me', 'of no concern/of concern to me', 'irrelevant/relevant to me', 'means nothing/means a lot to me', 'doesn't matter/matters to me', and 'insignificant/significant to me'. Houston and Walker (1996) found the scale was reliable (Cronbach's $\alpha=.99$ ).

\section{Health value}

Adopted from Lau et al. (1986), health value was measured using four 7-point Likert-type items: (i) "If you don't have your health, you don't have anything"; (ii) "There are many things I care about more than my health"; (iii) "Good health is of only minor importance in a happy life"; and (iv) "There is nothing more important than good health". Cronbach's $\alpha$ for the scale was .77 found in a health study (Armitage, Norman, \& Conner, 2002).

Two of the four items were negatively worded and reverse-scored (i.e., item 2 and item 3). The mean of the four items scores was used for analyses. 


\subsubsection{Demographics}

Demographic factors such as gender and alcohol use disorder are often used in antidrinking messages (e.g., Treeby \& Bruno, 2012). As a result, they have also been incorporated into the survey questionnaire of this study.

\section{Alcohol use disorder}

The Alcohol Use Disorders Identification Test (AUDIT, World Health Organization, 2007), was used to assess alcohol use disorder symptomatology in this binge drinking study.

The AUDIT is 10-item screening assessment used to identify hazardous and harmful alcohol consumption. The measure assesses three conceptual domains: frequency and quantity of alcohol intake ( 3 items), dependence indicators (3 items), and adverse alcohol use-related consequences (4 items) (see Appendix 3. Questionnaire). Responses to each question are scored from 0 to 4 , giving a maximum possible score of 40 . Higher scores on the AUDIT are indicative of progressively more hazardous drinking and an increasing likelihood of dependence. The AUDIT demonstrated good internal consistency with Cronbach's $\alpha=.80$ (Treeby \& Bruno, 2012).

In this study, alcohol use disorders were categorised into non- or low-risk (AUDIT total score $=0-7)$ and at-risk or high-risk drinkers (AUDIT total score $=8-40$ ) (Reinert $\&$ Allen, 2007).

\subsection{Data Analysis and Hypothesis-Testing Procedures}

Structural Equation Modelling (SEM) is widely used by researchers in marketing and consumer research (Bagozzi \& Baumgartner, 1994; Baumgartner \& Homburg, 1996; Hershberger, 2003). The use of SEM yields benefits not possible with first-generation statistical methods (e.g., correlation analysis, exploratory factor analysis, multiple regression, and ANOVA). One important benefit is that the types of error confounding first-generation procedures can be taken into account in the analysis (Chin, 1998; Iacobucci, 2009). Random or measurement errors in indicators can be modelled and estimated explicitly. Systematic or method errors can also be represented. Other advantages of SEM include the provision of methods to assess construct validity in broader and deeper ways than possible with traditional correlation analyses, and ways to 
correct for systematic bias in tests of substantive hypotheses (Bentler \& Stein, 1992). Use of SEM helps researchers to be more precise in the specification of hypotheses and operationalisation of constructs (Bagozzi \& Yi, 2012). It also takes into account the reliability of measures in tests of hypotheses in ways going beyond the averaging of multimeasures of constructs.

Generally, there are two types of SEM techniques applied in marketing literature including covariance-based SEM (CB-SEM), using software LISREL, AMOS, Mplus and variance-based partial least squares SEM (PLS-SEM), using software SmartPLS, WarpPLS, PLS-Graph. CB-SEM is more suitable for studies emphasizing theory testing and confirmation, while PLS-SEM is more appropriate for research focusing on theory development and prediction (Hair, Sarstedt, Ringle, \& Mena, 2012).

CB-SEM was conducted using the statistical analysis programme AMOS. The objective of covariance-based SEM is to show that the null hypotheses - the assumed research model with all its paths - is insignificant, meaning that the complete set of paths as specified in the model being analysed is plausible, given the sample data. In other words, it is conducted to show that the operationalisation of the theory under examination is corroborated and not disconfirmed by the data (Bollen, 1984; Hair, Anderson, Tatham, \& Black, 1998).

A number of data analysis techniques were performed following data collection. Data summaries were analysed using the statistical software SPSS. The scales used in the current study were based on existing literature; therefore, a confirmatory factor analysis (CFA) was performed. This was done using SEM to assess convergent and discriminant validity. Besides SEM, analysis of covariance (ANCOVA) was also used for hypothesis testing - examining the relationships among the constructs.

\subsection{Chapter Summary}

This chapter discussed the methods used to test the conceptual model including the experimental design, stimulus development and pretesting, sample and data collection process, and measurement development. The data analysis process using several advanced techniques of SEM and ANCOVA was also discussed in this chapter. The results of data analysis are presented in the next chapter along with evidence to support or refute each hypothesis. 


\section{Chapter 5. Data Analysis and Results}

\subsection{Introduction}

This chapter presents a detailed analysis of the relevant data collected from the main survey conducted in New Zealand. The chapter begins with the description of sample characteristics, the preliminary data analysis, the measurement model evaluation, the structural model estimation, and the results of the hypotheses testing.

\subsection{Main Survey}

\subsubsection{Description of Responses}

The main survey was made available from March to May 2016. The survey link was posted on several Victoria University of Wellington's 100 and 200 level courses Blackboard site and social network sites (e.g., Subjects Wanted ${ }^{4}$, Vic Deals ${ }^{5}$, New Zealand Universities’ Facebook).

Response statistics for the main survey are presented in Table 5.1. The survey yielded 532 responses, of which 460 passed the screening test as the respondents were undergraduate students. Of the 460 qualified respondents, 159 returned incomplete surveys with the majority of them not answering the primary questions related to the measurement model, therefore they were excluded from the analysis. The remaining 301 completed surveys represented a $65.4 \%$ completion rate or a $34.6 \%$ dropout rate (proportion of respondents who did not complete among all qualified respondents). The dropout rate appears high in comparison to individually targeted web surveys, which use panels or other lists to directly recruit respondents, with an average of about $15 \%$; but similar to general web surveys, which mostly recruit respondents through web-based announcements, with an average of about $30 \%$ (Galesic, 2006).

Respondents ranged in age from 16 to 30 years $(M=20.07, S D=2.19)$ with $59.0 \%$ of the sample being female and $41.0 \%$ male. This indicates that the survey attracted both genders equally. Furthermore, the majority of the respondents were New Zealanders

\footnotetext{
${ }^{4}$ Subjects Wanted (http://www.subjectswanted.co.nz) is a website for free listing of subjects wanted for researchers based at the University of Canterbury.

${ }^{5}$ Vic Deals (https://www.facebook.com/groups/vicdeals) is a Facebook group page for local trading of goods and free listing of rooms/flatmates wanted for students at Victoria University in Wellington.
} 
(77.4\%) with $86.5 \%$ of them European, 6.8\% Māori, 3.4\% Asian, and 3.3\% Pacific peoples, Middle Eastern and others. This shows that the sample gathered was homogeneous and in line with the targeted sample frame.

Table 5.1. Response Statistics from Main Survey

\begin{tabular}{lcl}
\hline Responses & N & \\
\hline Total respondents & 532 & \\
Screening test & 72 & \\
Qualified & $\mathbf{4 6 0}$ & \\
Incomplete & 159 & (dropout rate $=34.6 \%)$ \\
Valid respondents & $\mathbf{3 0 1}$ & (completion rate $=65.4 \%)$ \\
\hline
\end{tabular}

Table 5.2. Sample Characteristics

\begin{tabular}{lccc}
\hline Risk level & N & AUDIT total score & $\%$ \\
\hline Non- or low-risk & 136 & $0-7$ & 45.2 \\
At-risk or high-risk & 165 & $8-40$ & 54.8 \\
Total & $\mathbf{3 0 1}$ & & $\mathbf{1 0 0 . 0}$ \\
\hline Gender & $\mathbf{N}$ & Mean & SD \\
\hline Male & 120 & 10.93 & 8.051 \\
Female & 181 & 8.20 & 5.951 \\
\hline
\end{tabular}

Of the 301 valid respondents, $45.2 \%$ indicated that they are considered non- or low-risk drinkers, but $54.8 \%$ considered themselves at-risk or high-risk. Interestingly, $8.0 \%$ of the sample of undergraduate students stated that they are almost certainly dependent drinkers (AUDIT total score $\geq 20)$. Overall, it shows that males $(M=10.93, S D=8.05)$ drank more heavily than females $(M=8.20, S D=5.95)$ as indicated by AUDIT total score, $t(299)=3.374, p=.002($ see Table 5.2) .

\subsubsection{Distribution of Manipulation Type}

The main survey consists of eight treatment groups with a control group. Participants were randomly assigned to either a treatment group receiving a manipulated ad with a relevant questionnaire or control group receiving a specially designed control group questionnaire without an ad. 
It is clear that participants randomly assigned to either a treatment or a control group means that any differences in the comparison of two groups could be attributed to the effects of the treatment (Davis, 1997). Given that forced exposure to the stimulus ad or the viewing situation in this experiment is clearly different than that of one's home, this threatens external validity but has a higher degree of control over extraneous variables, enhances internal validity, and responses can thus be obtained more quickly and costs are lower than the 'real world' experiment.

Table 5.3. Distribution of Manipulation Type

\begin{tabular}{lcc}
\hline Manipulation & N & $\%$ \\
\hline Self-referenced guilt & 26 & 8.6 \\
Other-referenced guilt & 37 & 12.3 \\
Internal guilt & 34 & 11.3 \\
External guilt & 33 & 11.0 \\
Self-referenced shame & 35 & 11.6 \\
Other-referenced shame & 38 & 12.6 \\
Internal shame & 29 & 9.6 \\
External shame & 34 & 11.3 \\
Sub-total & $\mathbf{2 6 6}$ & $\mathbf{8 8 . 4}$ \\
Control (no manipulation) & $\mathbf{3 5}$ & $\mathbf{1 1 . 6}$ \\
Total & $\mathbf{3 0 1}$ & $\mathbf{1 0 0 . 0}$ \\
\hline
\end{tabular}

There was a mostly even distribution of responses for each experimental group (see Table $5.3)$.

\subsubsection{Manipulation Checks}

\subsubsection{Self-referencing}

There was a significant difference between self-reference and other-reference with regards to self-referencing, $t(134)=-2.624, p=.010$. That is, the mean score of yourself is significantly lower $(M=2.74)$ than others $(M=3.59)$. Thus, the self-referencing manipulation was effective.

\subsubsection{Sources of evaluation}

The difference between internal and external source in evaluation source ratings was significant, $t(128)=-4.825, p=.000$. That is, the mean score of yourself is significantly 
lower $(M=3.67)$ than others $(M=5.09)$. The evaluation source manipulation was also effective.

\subsubsection{Intensity level of emotional appeal}

\section{Guilt}

The differences across four types of guilt appeal in terms of felt guilt were not significant, $F(3,126)=1.632, p=.185$. As predicted, guilt appeal resulted in significantly more felt guilt $\left(M_{\mathrm{G}}=3.95\right)$ than felt shame $\left(M_{\mathrm{S}}=3.63\right), t(128)=2.636, p=.009$.

Furthermore, it was expected that across gender they would not significantly differ in terms of felt guilt. Results also reveal that the difference between males and females was not significant, $t(130)=-.843, p=.596$. Therefore, the guilt appeal manipulation was effective.

\section{Shame}

The differences across four types of shame appeal in terms of felt shame were also not significant, $F(3,132)=1.222, p=.304$. Similar to the guilt appeal pattern, shame appeal resulted in significantly more felt shame $\left(M_{\mathrm{S}}=4.38\right)$ than felt guilt $\left(M_{\mathrm{G}}=4.17\right), t(134)=$ $1.981, p=.045$.

As expected, the difference between males and females in terms of felt shame was not significant, $t(134)=-1.449, p=.150$. Thus, the shame appeal was also effective.

\section{Guilt versus Shame}

Furthermore, it was expected that the guilt and shame appeals would not significantly differ in terms of felt respective emotion. Results reveal that the difference between felt emotion of guilt and shame was not significant, $t(264)=-1.908, p=.058\left(M_{\mathrm{G}}=3.95, M_{\mathrm{S}}\right.$ $=4.38$ ). Therefore, a comparison between guilt and shame appeals can be made without concern about emotional appeal intensity level.

\subsubsection{Effects of manipulation}

It was expected that the treatment groups would manifest significantly more emotion arousal than did the control group as a result of the treatment. Results reveal that the differences in emotion arousal between the treatment groups (receiving a guilt appeal or shame appeal ad) and the control group (receiving no ad) were significant ( $p$ s $<.005)$. 
That is, the mean score of the treatment groups is significantly higher than that of the control group: $M_{\mathrm{G}}=3.09$ for guilt appeal compared with the control $M_{\mathrm{C}}=1.97$ and $M_{\mathrm{S}}=$ 3.04 for shame appeal compared with the control $M_{\mathrm{C}}=2.29$. This suggests that a manipulation check was successful.

In all, the manipulated ads worked effectively in the main survey with both male and female undergraduate students in New Zealand.

\subsection{Pre-SEM Analysis Treatments and Evaluations}

Prior to conducting the SEM analyses, the mean variable scores, missing data, reverse coding requirements, common method variance and data normality were checked against the data. These are discussed in turn in the following sections.

\subsubsection{Variable Means}

Table 5.4 presents means and standard deviations (SD) for all of the continuous variables used in the models. As shown, almost all of the variable mean values were close to the median values.

\subsubsection{Missing Data and Negative Questions}

Within the completed survey, there were no missing data. This was achieved because the main survey employed the force response tool to ensure respondents answered all the questions within each section before proceeding to the next one. Two negatively worded questions were used in the main survey; these were items measuring the control variable health value (i.e., item 2 and item 3, see Section 4.3.5.5). Following a suggestion by Allen and Bennett (2010, p. 9) for a production of "meaningful total or average", the responses to these items were reversed.

After the establishment of no missing data and treatment of any negatively worded questions, the data was ready to be analysed.

\subsubsection{Common Method Variance}

The present study checked the main survey's data for variances that may be caused by measurement method instead of the constructs the method intended to measure (Chang, Van Witteloostuijn, \& Eden, 2010; Podsakoff, MacKenzie, Lee, \& Podsakoff, 2003). The main survey attempted to avoid the issues of Common Method Variance (CMV) by 
ensuring respondents were not able to associate questions with the constructs that they represent so as to create answers they felt would produce better results (Chang et al., 2010). Also, the main survey contained no double-barrelled items and no unfamiliar and/or undefined concepts and jargons (Podsakoff et al., 2003).

There are some statistical remedies to check for CMV which include Harman's single factor test; partial correlation procedures such as partialling out (i) social desirability, (ii) general factor score, and (iii) a marker variable. To examine CMV, Harman's single factor and marker variable tests are employed (Podsakoff et al., 2003; Rönkkö \& Ylitalo, 2011).

\subsubsection{Harman's single factor test}

The most widely used statistical check to assess CMV is Harman's single factor test (Podsakoff et al., 2003). CMV is presumed to exist if un-rotated exploratory factor analysis (EFA) results in any single factor explaining the majority of variance in the variables (more than 50\%). Accordingly, an un-rotated EFA resulted in the first factor explaining $35.84 \%$ of the variance and it required two factors to reach $50.53 \%$ (see Appendix 6). This result shows that no single factor (one general factor) accounts for the majority of the covariance among the items in the survey, indicating CMV was unlikely to be a problem in this study.

\subsubsection{Marker variable test}

A more stringent statistical check for CMV is marker variable test (Williams, Hartman, \& Cavazotte, 2010). CMV is assessed based on the correlation between the marker variable and a priori unrelated theoretically constructs. In this study, the construct Health message exposure (HME) identified as being theoretically unrelated to at least one of the other constructs in the model, e.g., Regulatory focus (ProRF, PreRF) and Coping response (CR); thus was selected as a marker (Lindell \& Whitney, 2001). Accordingly, all correlations between the marker and the conceptually unrelated constructs were nonsignificant at $95 \%$ confidence level (see Appendix 7).

In addition, according to Bagozzi's method, CMV occurs when the highest correlation between constructs is more than 90 (Bagozzi, Yi, \& Phillips, 1991). As shown in Table 5.12, the highest correlation between constructs (i.e., Emotional appeal and Emotional arousal) is .56. Therefore, it appears that CMV is not a concern for this study. 


\subsubsection{Normality of the Data}

An important assumption in conducting the two steps of SEM analyses (CFA and hypotheses testing of the structural model) is that the data is normally distributed (Allen \& Bennett, 2010; Arbuckle, 2013; Hair et al., 2010). As sample data may be used to represent the normality of the sampling distribution (principle of central limit theorem) (Field, 2013), the present study conducted normality analysis on the main survey data. Data normality can be analysed using skewness and kurtosis tests (Byrne, 2010), where the former measures the symmetry of the distribution of the data and the latter measures the shape of the distribution of the data (Field, 2013).

Table 5.4. Descriptive Statistics and Assessment of Normality of the Data

\begin{tabular}{lccccc}
\hline Variables (Constructs) & Mean & Median & \multicolumn{1}{c}{ SD } & Skewness & Kurtosis \\
\hline Guilt appeal (GAp) & 3.95 & 4.00 & 1.844 & .026 & -1.055 \\
Shame appeal (SAp) & 4.38 & 5.00 & 1.848 & -.223 & -1.087 \\
Guilt arousal (GAr) & 3.09 & 3.08 & 1.676 & .324 & -1.038 \\
Shame arousal (SAr) & 3.04 & 2.65 & 1.482 & .436 & -.936 \\
Coping response (CR) & 2.05 & 2.00 & .935 & -.095 & -1.856 \\
Message compliance (MC) & 3.52 & 3.67 & 1.548 & .211 & -.570 \\
Independent self-construal (IndSC) & 4.95 & 4.91 & .977 & -.031 & -.107 \\
Interdependent self-construal (IntSC) & 4.71 & 4.83 & .884 & -.448 & .803 \\
Promotion focus (ProRF) & 5.47 & 5.56 & 1.075 & -.977 & 1.760 \\
Prevention focus (PreRF) & 4.47 & 4.56 & 1.176 & -.261 & -.720 \\
Personal cultural orientation (PCO) & 4.01 & 4.00 & 1.289 & -.041 & -.257 \\
\hline
\end{tabular}

* Except for CR using a 3-point scale, all variables use a 7-point scale.

Normally distributed data is indicated by skewness and kurtosis figures that are as close as possible to the value of zero and an extreme departure from this value will signal a set of data that is not normally distributed (Curran, West, \& Finch, 1996). Burns and Burns (2008) suggested that an extreme departure is where the values are above \pm 2 for skewness and \pm 3 for kurtosis.

Regarding critical statistics for normal distribution, the data used in the analysis were considered as normally distributed (see Table 5.4, more in Appendix 5). Values of skewness varied from -1.452 (Int1) to $+1.136(\mathrm{SAr} 3)^{6}$. Values for kurtosis ranged from -

\footnotetext{
${ }^{6}$ See explanations of acronyms for items in Appendix 4.
} 
1.856 (CR1) to +2.360 (Int1). These values of skewness and kurtosis indicated that the measured items were not subject to skewness or kurtosis. The measured items may be taken to be normally distributed, and were thus retained for further multivariate analysis.

\subsubsection{Non-Response and Response Bias}

Non-response takes place when a sampled individual refuses to participate in a survey. The bias occurs when answers to questions differ among the observed and nonrespondent units. The common practice to check non-response bias is to compare two wave samples (e.g., initial sample survey and later sample survey). A comparison was made, reminiscent of Mittal, Kumar and Tsiros' (1999) study, between the first 51 responses and the last 51 responses. The ratings on all key construct scales were statistically the same (all $p \mathrm{~s}>.05$ ) (see Appendix 8). Thus, it is reasonable to conclude that the data used in this study is not biased.

\subsubsection{Sample Size and Power}

The two-stage SEM method requires a specific estimation of a sample size that would be sufficient to produce a satisfactory model fit for a number of indices tests (Hu \& Bentler, 1999). Byrne (2010) suggested the use of Hoelter's (1983) Critical N (CN) index to determine the adequacy of sample size for SEM analysis. Hoelter (1983) proposed an index value above 200 to be indicative of a model that is adequately representing the sample data. Analysis of the present study's results showed that $\mathrm{CN}$ values at .05 and .01 confidence intervals for the hypothesised model were 139 and 144 respectively. Following Hoelter's (1983) criterion, the size of the main survey's sample ( $n=266$, excluding control group) was deemed satisfactory.

\subsection{Critical Statistical Values Applied in the Study}

The literature of SEM and related studies recommends a two-step model building approach (Anderson \& Gerbing, 1988; Hair et al., 2010; Schumacker \& Lomax, 2004). The first step involved EFA for untested new scales, and CFA for pre-existing validated scales, to purify and validate the measures. The second step involved building and testing the structural model. Since the current study used existing scales to measure different constructs, CFA was used to confirm and reduce the number of items from the constructs. AMOS version 23 software was used to conduct the CFA and test the model (Byrne, 2010). 


\subsubsection{Confirmatory Factor Analysis and Model Fit Statistics}

Measurements for the survey data were examined with CFA. The measurements were assessed via: $(i)$ the construct validity in terms of convergent validity and discriminant validity, and (ii) the goodness-of-fit of proposed measurement models (the overall model fit).

Table 5.5 presents critical values for assessing the measurement and structural model. Convergent validity is met if absolute standardised loading is greater than .50 (Hair et al., 2010), average variance extracted (AVE) greater than .50 (Fornell \& Larcker, 1981), and construct reliability (C.R.) greater than .70 (Hair et al., 2010). An observed variable's squared multiple correlation $\left(R^{2}\right)$ value reflects the extracted variance that is explained by the underlying latent variable (Fornell \& Larcker, 1981). In the context of factor analysis (e.g., CFA), this corresponds to the observed variable's reliability or communality. In marketing research, an ideal rule-of-thumb for $R^{2}$ is .75 (Grewal, Cote, \& Baumgartner, 2004), which indicates that the latent variable is explaining a substantial amount of the variation in the observed variable. An $R^{2}$ value of .50 , however, is considered to be moderate as it is explaining at least half of the variation in the observed variable with the other half being error variance, which is deemed acceptable (Hair et al., 2010).

Discriminant validity can be evaluated by examining the cross-loading (Chin, 1998), Fornell and Larcker criterion (Fornell \& Larcker, 1981), and the Heterotrait-monotrait (HTMT) ratio of correlations (Henseler, Ringle, \& Sarstedt, 2015). The discriminant validity is confirmed subject to no cross loading of an item on more than one variable, AVE which exceeds any squared inter-construct correlations $\left(R^{2}\right)$ associated with that variable, and HTMT which is below .85 (HTMT.85) (Henseler et al., 2015; Voorhees, Brady, Calantone, \& Ramirez, 2016). The HTMT ratio calculates the average correlation of items across latent variables measuring different constructs (i.e., heterotraitheteromethod correlations), relative to the correlations of the item within the same latent variable (i.e., monotrait-heteromethod correlations).

For goodness-of-fit, Bagozzi and Yi (2012) suggest ideal Tucker-Levis Index (TLI) and Comparative Fit Index (CFI) are expected to be greater than .92, .93, respectively. Root Mean Square Error of Approximation (RMSEA) and Standardised Root Mean Square Residual (sRMR) are both expected to be lower than .07. Normed Chi-square $\left(\chi^{2} / d f\right)$ is expected to be lower than 3.0 (Kline, 2016). 
Table 5.5. Rules of Thumb for CFA and Model Fit Statistics

\begin{tabular}{|c|c|c|c|}
\hline CFA output & & Values & Sources \\
\hline \multicolumn{4}{|l|}{ Construct Validity } \\
\hline \multicolumn{4}{|l|}{ Convergent validity } \\
\hline Standardised loadings & & $\geq .50$, ideally $\geq .70$ & Hair et al. (2010) \\
\hline Average variance extracted & AVE & $\geq .50$ & Fornell and Larcker (1981) \\
\hline Construct reliability & C.R. & $\geq .60$, ideally $\geq .70$ & Hair et al. (2010) \\
\hline \multicolumn{4}{|l|}{ Discriminant validity } \\
\hline No cross loading & & & Chin (1998) \\
\hline Fornell-Larcker criterion & & AVE $>R^{2}$ & Fornell and Larcker (1981) \\
\hline HTMT ratio & & $\leq .85$ & Henseler et al. (2015) \\
\hline Bivariate correlation $\left(\mathrm{R}^{2}\right)$ & & $<.75$ & Grewal et al. (2004) \\
\hline \multicolumn{4}{|l|}{ Measurement Model Fit } \\
\hline Chi-square $\left(x^{2}\right)$ & CMIN & & \\
\hline Degree of freedom & DF & & \\
\hline Normed Chi-square $\left(x^{2} / d f\right)$ & CMIN/DF & $\leq 3.0$ & Kline (2016) \\
\hline \multicolumn{4}{|l|}{ Incremental fit indices } \\
\hline Tucker and Lewis Index & TLI & $\geq .92$ & Bagozzi and Yi (2012) \\
\hline Comparative Fit Index & $\mathrm{CFI}$ & $\geq .93$ & Bagozzi and Yi (2012) \\
\hline \multicolumn{4}{|l|}{ Absolute fit measures } \\
\hline Standardised Root Mean Square Residual & sRMR & $\leq .07$ & Bagozzi and Yi (2012) \\
\hline Root Mean Square Error of Approximation & RMSEA & $\leq .07$ & Bagozzi and Yi (2012) \\
\hline
\end{tabular}

Suggested by Fornell and Larcker (1981), AVE and C.R. were calculated, as shown in Equations 5.1 and 5.2 below, respectively, simply by using standardised loadings $\left(L_{i}\right)$ and error variance terms for a construct $\left(1-L_{i}^{2}\right)$ :

$$
\begin{gathered}
A V E=\frac{\sum_{i=1}^{n} L_{i}^{2}}{n} \\
C . R .=\frac{\left(\sum_{i=1}^{n} L_{i}\right)^{2}}{\left(\sum_{i=1}^{n} L_{i}\right)^{2}+\left(\sum_{i=1}^{n}\left(1-L_{i}^{2}\right)\right)}
\end{gathered}
$$




\subsubsection{Modification and Specification}

A maximum-likelihood (ML) method has been used to examine the covariance matrix of the items. A summary of the modification and specification and their acceptable thresholds are provided in Table 5.6.

Table 5.6. Modifications and Specifications

\begin{tabular}{lccc}
\hline CFA output & & Values & Sources \\
\hline Modifications, specifications & & & \\
Standardised residuals & S.R. & $>2.58$ & Hair et al. (2010) \\
Modification Index & MI & Error covariance $>20$ & Hair et al. (2010) \\
Critical ratio & c.r. & $\geq 1.96$ & Hair et al. (2010) \\
\hline
\end{tabular}

The associated standard errors (S.E.) for each parameter estimate are analysed to measure the level of precision with which the parameter was estimated (Byrne, 2010). Lastly, all c.r. values (found by dividing standardised parameter estimates by their respective S.E.) of more than 1.96 are significant at the .05 level. This indicates that the null hypothesis (e.g., the baseline model), a model prior to any modifications and specifications (McDonald \& Ho, 2002), can be rejected (Byrne, 2010).

\subsection{Confirmatory Factor Analysis and Measurement Model}

A CFA approach was used to test the factorial validity of the hypothesised measurement model before evaluating the structural (theoretical) model (Anderson \& Gerbing, 1988; Hair et al., 2010; Schumacker \& Lomax, 2004).

For the measurement model, the study firstly validates the main factors (independent/mediating and dependent variables), then the explanatory factors (moderating variables), and eventually the overall model, including all these variables (Hair et al., 2010).

The study's main measurement model includes two models: guilt and shame. They were tested separately due to guilt and shame being distinct emotions and the respective emotional arousal was measured by a different scale (12 items for guilt arousal and 10 items for shame arousal). Each model consists of four factors/constructs (latent variables) including guilt appeal (GAp)/shame appeal (SAp), guilt arousal (GAr)/shame arousal (SAr), coping response (CR), and message compliance (MC). The measurement model of 
explanatory variables in this study includes five latent variables: independent selfconstrual (IndSC), interdependent self-construal (IntSC), promotion focus (ProRF), prevention focus (PreRF), and personal cultural orientation (PCO) ${ }^{7}$.

While possible, good fit for the models to the data is rarely achievable without some form of modification and specification, as in the case of this analysis where there was evidence of misfit across all latent variables. It is thus apparent that some modification and specification is needed to generate a model that better represents the sample data (Singh \& Billingsley, 1998). In conducting modification several steps are taken, including a review of S.R. and MI and parameter change statistics for covariance and regression weights (Byrne, 2010) (see their cut-points in Table 5.6).

\subsubsection{Measurement of Main Factors}

The main measurement model for guilt and shame included all items related to main constructs. The original measurement model, including all 17 items for guilt and 15 items for shame, was tested in the respective sample. Each measurement model included two multi-item constructs (GAr/SAr and MC) and two single-item constructs (GAp/SAp and CR) (see Figure 4.2). Single-item constructs were handled by fixing their loading to 1.0, forcing their error variance to zero, and leaving their variances free to be estimated (Hair et al., 2010).

\subsubsection{Guilt model}

The original measurement model for guilt used for CFA did not provide a reasonable fit to the data: $\chi^{2} / d f=1.99 ; \mathrm{TLI}=.93 ; \mathrm{CFI}=.94 ; \mathrm{sRMR}=.047$; RMSEA $=.088$. Although all factor loadings were greater than .50, one item (i.e., MC1) did not meet minimum communality $(>.40)$ considered to be eliminated. After reviewing with respect to theoretical basis (e.g., content) and given that findings from the shame model revealed similar inadequacies in its measurement of this MC item (i.e., low factor loading, see Section 5.5.1.2 below), the item was subsequently removed from the final model (e.g., Anderson \& Gerbing, 1988).

In examining the S.R., not all values exceeded the cut-point of 2.58. Therefore, no further items were considered for elimination from the measurement model. After modification,

\footnotetext{
${ }^{7}$ See List of Abbreviations for explanations of acronyms.
} 
however, the measurement model with the remaining 16 items still did not meet the goodness-of-fit criteria: $\chi^{2} / d f=1.99 ; \mathrm{TLI}=.94 ; \mathrm{CFI}=.95 ; \mathrm{sRMR}=.036 ; \mathrm{RMSEA}=$ .088. In this case, these unsatisfactory results are due to the adopted scale of guilt arousal consisting of more than five indicators (Bagozzi \& Baumgartner, 1994). Bagozzi \& Baumgartner (1994) noted that even with adopting a well-developed scale, "disappointing results are all but guaranteed" (p. 388) if the number of indicators goes much beyond five.

Table 5.7. Model Fit Statistics for the Measurement Model - Guilt

\begin{tabular}{lrrrrc}
\hline Guilt Model & $\mathbf{X}^{2} / \boldsymbol{d} \boldsymbol{f}$ & \multicolumn{1}{c}{ TLI } & \multicolumn{1}{c}{ CFI } & \multicolumn{1}{c}{ sRMR } & RMSEA \\
\hline Original model & 1.99 & .93 & .94 & .047 & $.088(.071-.104)$ \\
Final model & .69 & 1.01 & 1.00 & .013 & $.000(.000-.070)$ \\
\hline
\end{tabular}

As a way of improving the model fit as suggested by Bagozzi and Baumgartner (1994), a single composite of measures for the construct is formed prior to submitting the data to a structural equations programme or the scale is divided in half or, better yet, thirds and these sub-scale composites are used as multiple indicators of the construct of interest. Thus, the scale of guilt arousal was divided into three summated sub-scales. Accordingly, GAr consisting of GArI (averaged GAr1, GAr4, GAr8, GAr12), GArII (GAr5, GAr6, GAr10, GAr11), and GArIII (GAr2, GAr3, GAr7, GAr9) was formed based on a combination of high loading items with low loading items to create a well-balanced scale (Little, Cunningham, Shahar, \& Widaman, 2002).

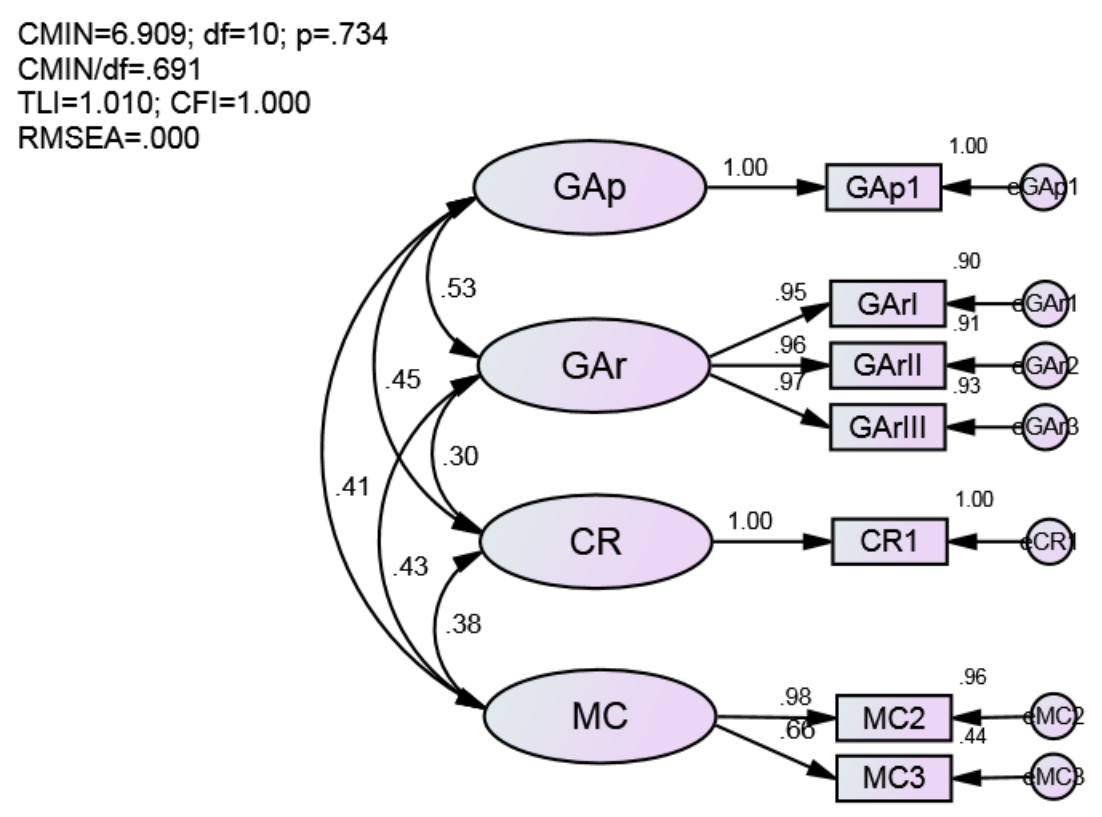

Figure 5.1. Final Measurement Model for Guilt 
By re-presenting the measurement model with seven items, including three summated items (one individual item MC1 removed), the final measurement model for the guilt model provided the superior fit for the data: $\chi^{2} / d f=.69$; TLI $=1.01 ; \mathrm{CFI}=1.00$; $\mathrm{sRMR}=$ .013 ; RMSEA $=.000$ (shown on the last row of Table 5.7, CFA model in Figure 5.1). The fit of the measurement model is deemed acceptable.

\subsubsection{Shame model}

The original measurement model for shame used for CFA did not provide a reasonable fit to the data: $\chi^{2} / d f=3.49 ; \mathrm{TLI}=.82 ; \mathrm{CFI}=.86 ;$ sRMR $=.071 ; \mathrm{RMSEA}=.136$. Almost all factor loadings were greater than .50 with only one item (i.e., MC1) that loaded less than the threshold. The findings were consistent with the guilt model, especially for the MC scale item MC1; subsequently, this item was removed from the model.

Table 5.8. Model Fit Statistics for the Measurement Model - Shame

\begin{tabular}{lccccc}
\hline Shame Model & $\mathbf{X}^{2} / \boldsymbol{d} f$ & TLI & CFI & sRMR & RMSEA \\
\hline Original model & 3.49 & .82 & .86 & .071 & $.136(.119-.153)$ \\
Final model & 1.45 & .98 & .99 & .026 & $.057(.000-.118)$ \\
\hline
\end{tabular}

After modification, the measurement model with the remaining 14 items still did not meet the goodness-of-fit criteria: $\chi^{2} / d f=3.98 ; \mathrm{TLI}=.81$; CFI $=.85 ; \mathrm{sRMR}=.069 ; \mathrm{RMSEA}=$ .148. As with guilt, these unsatisfactory results are due to the adopted scale of shame arousal consisting of more than five indicators (Bagozzi \& Baumgartner, 1994). To improve the model fit, the scale of shame arousal was divided into three summated subscales. Accordingly, SAr consists of SArI (averaged SAr1, SAr2, SAr3), SArII (SAr4, GAr5, GAr7), and SArIII (SAr6, SAr8, SAr9, SAr10). 


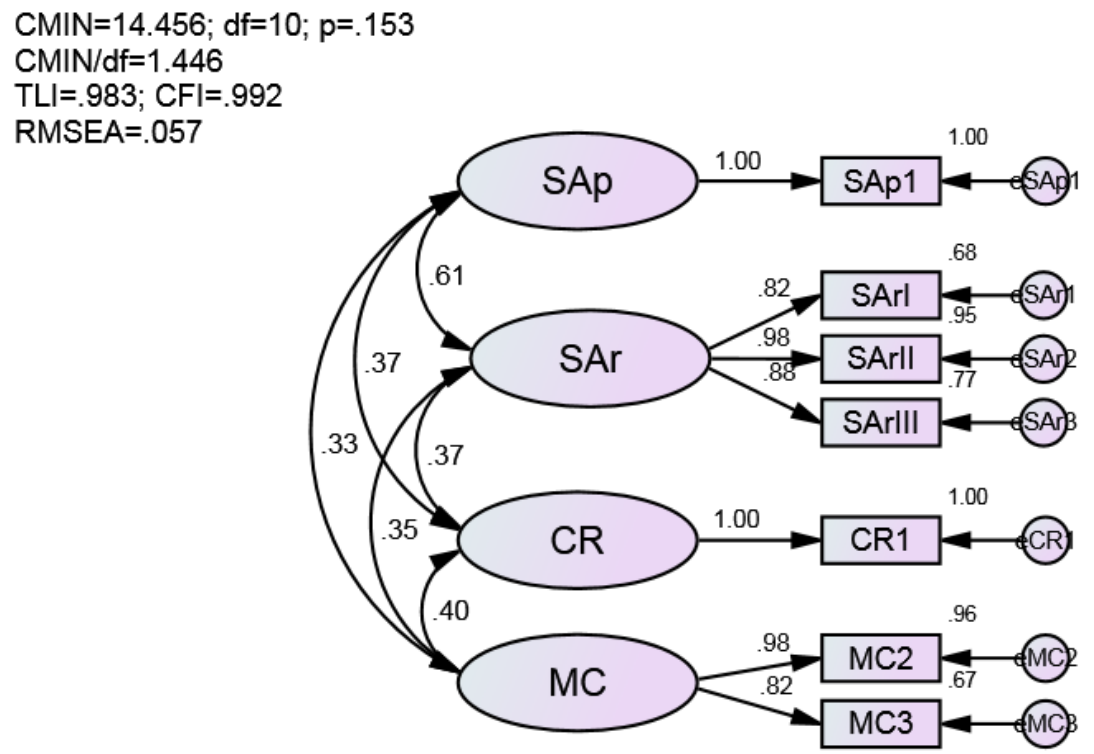

Figure 5.2. Final Measurement Model for Shame

By re-presenting the measurement model with seven items including three summated items (MC1 removed), the final measurement model for the shame model provided the superior fit for the data: $\chi^{2} / d f=1.45$; TLI $=.98$; CFI $=.99$; $\mathrm{sRMR}=.026$; RMSEA $=$ .057 (shown on the last row of Table 5.8, CFA model in Figure 5.2). The fit of the measurement model is deemed acceptable.

It is noted that guilt and shame models are now identical with seven individual/summated items: EAp1 (GAp1/SAp1); EAr1 (GArI/SArI), EAr2 (GArII/SArII), EAr3 (GArIII/ SArIII); CR1; and MC2, MC3. Thus, they could be nested within one model - the overall measurement model (Hair et al., 2010) to be validated (see Section 5.5.3.2).

\subsubsection{Measurement of Explanatory Factors}

The measurement model for explanatory factors, including five constructs (latent variables) was tested in the whole sample. All factors are multi-item constructs (see Figure 4.2). It is also noted that, unlike the main factors (exogenous/endogenous variables), the explanatory factors (grouping variables) are not modelled in the structural model.

The original measurement model used for CFA did not provide a reasonable fit to the data: $\chi^{2} / d f=2.45 ; \mathrm{TLI}=.75 ; \mathrm{CFI}=.76 ; \mathrm{sRMR}=.103 ; \mathrm{RMSEA}=.074$. 
Table 5.9. Model Fit Statistics for the Measurement Model - Explanatory Factors

\begin{tabular}{lrrrrc}
\hline Explanatory Model & $\mathrm{X}^{2} / d f$ & \multicolumn{1}{c}{ TLI } & CFI & \multicolumn{1}{c}{ sRMR } & RMSEA \\
\hline Original model & 2.45 & .75 & .76 & .103 & $.074(.070-.078)$ \\
Final model & 1.78 & .96 & .97 & .056 & $.054(.042-.066)$ \\
\hline
\end{tabular}

Following the guidelines suggested by Bagozzi and Yi (1988) and DeVellis (2016), CFA diagnostic measures, including factor loadings, MI, and S.R., served as criteria to improve the model fit as well as the construct validity. Although re-specification may be either theory- or data-driven, the ultimate objective is to find a model that is both substantively meaningful and statistically well fitting (Jöreskog, 1993). As demonstrated in Sections 5.5.2.1, 5.5.2.2, and 5.5.2.3 below, the modifications of the model were made based on both the theoretical integrity of these explanatory factors and methodological reasons.

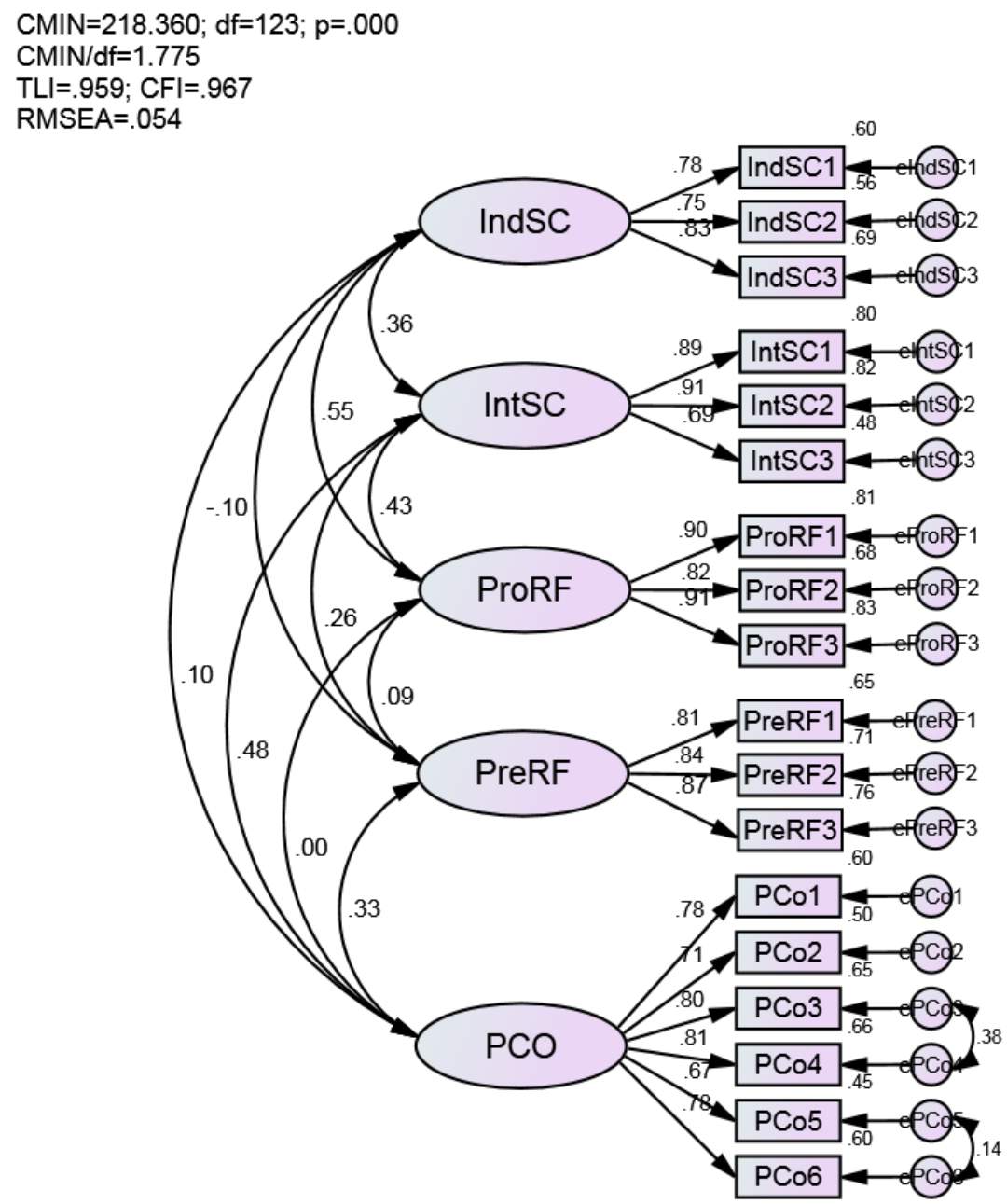

Figure 5.3. Final Measurement Model for Explanatory Factors 
Post modification, the final measurement model satisfactorily met the goodness-of-fit criteria: $\chi^{2} / d f=1.78 ; \mathrm{TLI}=.96 ; \mathrm{CFI}=.97 ; \mathrm{sRMR}=.056$; $\mathrm{RMSEA}=.054$ (shown on the last row of Table 5.9, CFA model in Figure 5.3).

\subsubsection{Self-construal}

The original measurement model for self-construal (SC) consists of 23 items: 11 items for independent SC (IndSC) and 12 items for interdependent SC (IntSC). Three items loaded less than .50 including Int4, Int11, and Int12 (IntSC). These three items were reviewed with respect to theoretical basis (e.g., content) and the context of their construct (e.g., convergent validity), and were subsequently removed from the measurement model (indicator items deleted from the measurement model are attached in Appendix 9, Anderson \& Gerbing, 1988). This modification of scale was acceptable (Anderson \& Gerbing, 1988; Bagozzi \& Baumgartner, 1994; Gaskin, 2012a). As Gaskin (2012a) suggested, when using an established scale with a large number of items (more than 10), it can be reduced to the 4-5 items that best capture the construct of interest as needed.

After modification, the measurement model with the remaining 20 items still did not meet the goodness-of-fit criteria: $\chi^{2} / d f=3.62$; TLI $=.73$; CFI $=.76$; $\mathrm{sRMR}=.094$; RMSEA $=$ .100. In the present case, these unsatisfactory results were due to the adopted scale consisting of more than five indicators (Bagozzi \& Baumgartner, 1994). To improve the model fit, as suggested by Bagozzi and Baumgartner (1994), the scale of SC was divided into six summated sub-scales. Accordingly, IndSC consists of IndSC1 (averaged Ind1, Ind3, Ind4, Ind11), IndSC2 (Ind5, Ind6, Ind7, Ind9), and IndSC3 (Ind2, Ind8, Ind10); IntSC consists of IntSC1 (Int2, Int6, Int8), IntSC2 (Int1, Int2, Int9), and IntSC3 (Int3, Int5, Int7).

By re-presenting the measurement model with six summated measurement items (three individual items removed), the final measurement model for SC provided the superior fit for the data: $\chi^{2} / d f=1.48 ; \mathrm{TLI}=.99 ; \mathrm{CFI}=.99 ; \mathrm{sRMR}=.035 ; \mathrm{RMSEA}=.042$.

Similar to Kim and Johnson (2014), a measure of dominant SC was created by subtracting the IntSC score from the IndSC score. That is, positive average difference scores reflected in IndSC and negative average difference scores reflected in IntSC. Participants were then classified as either IndSCs or IntSCs on the basis of the average 
difference score. Consequently, the dominant SC consists of 122 IndSCs (45.9\%) and 144 IntSCs $(54.1 \%)$.

\subsubsection{Regulatory focus}

The original measurement model for regulatory focus (RF) consists of 18 items: nine items for promotion focus (ProRF) and nine items for prevention focus (PreRF). Only one item loaded less than .50 (i.e., Pre1). S.R.s were much larger than 2.58 in absolute magnitude related to Pre9 (S.R. for Pre9 and Pro9 $=5.990$, Pro8 $=4.690$, Pro7 $=3.832$, and Pro5 $=3.187$ ). These two items were removed from the model after reviewing with respect to theoretical basis (e.g., content) and context of their construct (e.g., convergent validity) (e.g., Anderson \& Gerbing, 1988).

After modification, the measurement model with the remaining 16 items still did not meet the goodness-of-fit criteria: $\chi^{2} / d f=4.19 ; \mathrm{TLI}=.84 ; \mathrm{CFI}=.86 ; \mathrm{sRMR}=.096$; $\mathrm{RMSEA}=$ .110. Again, these disappointing results were due to the adopted scale having more than five indicators (Bagozzi \& Baumgartner, 1994). To improve the model fit, the scale of RF was divided into six summated sub-scales. Accordingly, ProRF consists of ProRF1 (averaged Pro5, Pro6, Pro8), ProRF2 (Pro2, Pro3, Pro4), and ProRF3 (Pro1, Pro7, Pro9); PreRF consists of PreRF1 (Pre4, Pre7, Pre8), PreRF2 (Pre3, Pre6), PreRF3 (Pre2, Pre5).

By re-presenting the measurement model with six summated measurement items (two individual items removed), the final measurement model for RF provided the superior fit for the data: $\chi^{2} / d f=1.80 ; \mathrm{TLI}=.99 ; \mathrm{CFI}=.99 ; \mathrm{sRMR}=.039 ; \mathrm{RMSEA}=.055$.

Following previous research (e.g., Lockwood et al., 2002), a measure of dominant RF was created by subtracting the PreRF score from the ProRF score. That is, positive average difference scores reflected in ProFC and negative average difference scores reflected in PreFC. Participants were then classified as either ProRFs or PreRFs on the basis of the average difference score. Accordingly, the dominant RF consists of 193 ProRFs (72.6\%) and 73 PreRFs (27.4\%).

\subsubsection{Personal cultural orientation}

The original measurement model for Personal cultural orientation (PCO) consisting of six items did not provide a reasonable fit to the data: $\chi^{2} / d f=4.29 ; \mathrm{TLI}=.94 ; \mathrm{CFI}=.97$; $\operatorname{sRMR}=.033 ; \mathrm{RMSEA}=.111$. All factor loadings were greater than .50 . To improve the 
model fit, the error terms were connected (suggested by MI > 20): ePCo3 <--> ePCo4, ePCo5 <--> ePCo6. Two sets of observed variables for PCO (shown in red in Appendix 4), do reflect similar but not identical questions in the survey. This was acceptable as they were supposed to measure one latent variable. Therefore, no items needed to be deleted; instead, correlations between the two sets of error terms were allowed (see correlation coefficients in Appendix 10) and this has improved the goodness-of-fit for the model to a satisfactory level: $\chi^{2} / d f=2.42 ; \mathrm{TLI}=.98 ; \mathrm{CFI}=.99 ; \mathrm{sRMR}=.020 ; \mathrm{RMSEA}=.073$.

The scale of PCO (collectivism-individualism) includes all six individual items measured with a 7-point Likert-type scale. Higher scores mean collectivist tendencies, whereas lower scores individualist tendencies. Participants were classified as either individualists (IndPCOs) or collectivists (ColPCOs) on the basis of the scale mid-point $(\mathrm{Mpt}=4.00)$ (Yoo \& Donthu, 2005). Accordingly, the dominant PCO consists of 144 IndPCOs $(54.1 \%)$ and 122 ColPCOs (45.9\%).

\subsubsection{Overall Measurement Model}

The measurement model, including all the main and explanatory factors for guilt and shame (two nested models), using the corresponding data, and the overall measurement model, including these two nested models, using the whole data, were then assessed.

\subsubsection{Nested models}

The final measurement model for both guilt and shame nested models satisfactorily met the goodness-of-fit criteria (see Table 5.10, CFA models in Appendices 11 and 12).

Table 5.10. Model Fit Statistics for the Final Measurement Model

\begin{tabular}{lccccc}
\hline Measurement Model & $\mathbf{X}^{2} / \boldsymbol{d} \boldsymbol{f}$ & TLI & CFI & sRMR & RMSEA \\
\hline Guilt model & 1.36 & .95 & .96 & .051 & $.052(.037-.066)$ \\
Shame model & 1.42 & .94 & .95 & .064 & $.056(.042-.069)$ \\
Overall model & 1.60 & .96 & .96 & .052 & $.048(.039-.056)$ \\
\hline
\end{tabular}

\subsubsection{Overall model}

The final overall measurement model for the nested guilt and shame models satisfactorily met the goodness-of-fit criteria: $\chi^{2} / d f=1.60 ;$ TLI $=.96 ; \mathrm{CFI}=.96 ;$ sRMR $=.052$; RMSEA $=.048$ (shown on the last row of Table 5.10, CFA model in Figure 5.4). 


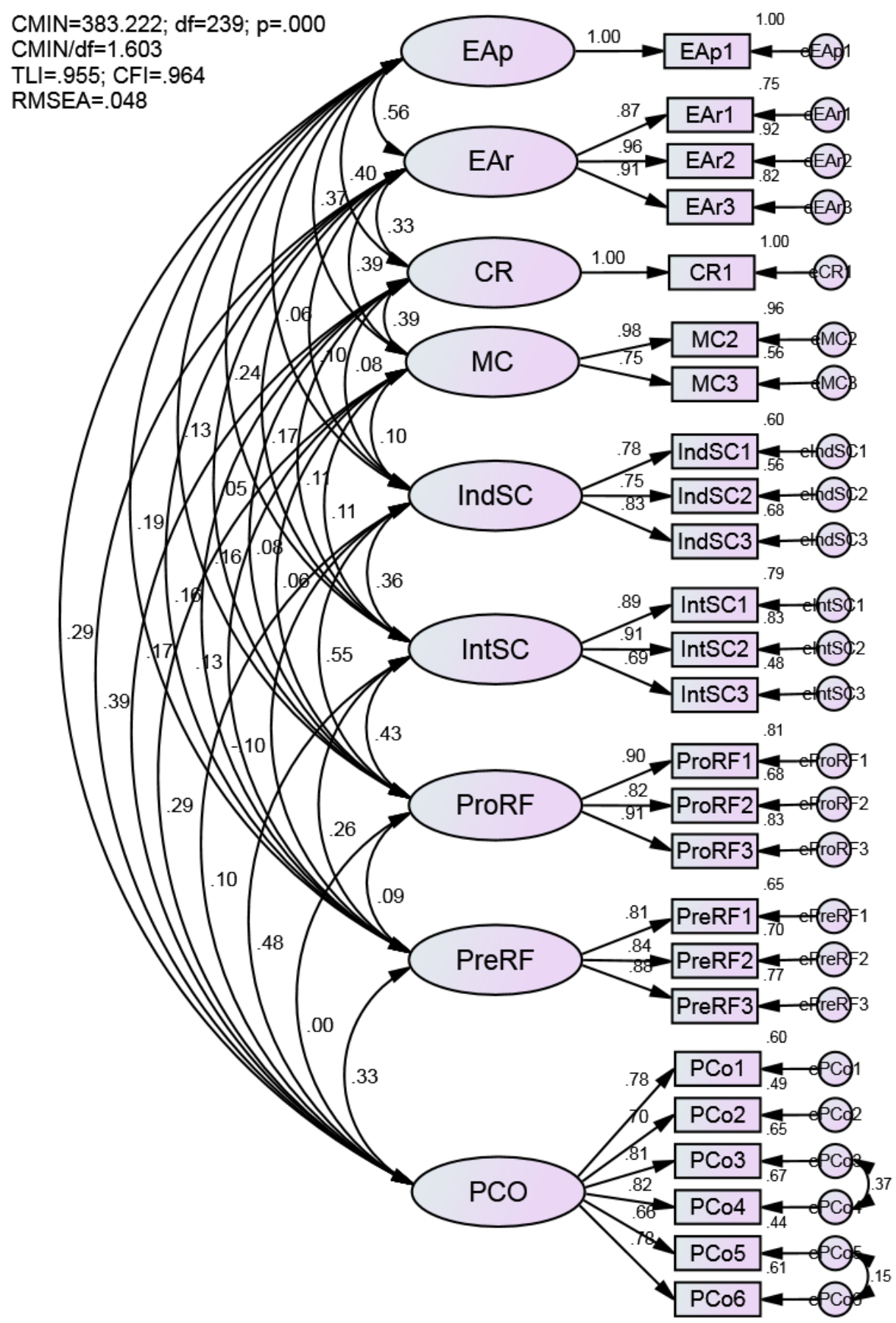

Figure 5.4. Final Overall Measurement Model

It is also noted that in reviewing the standardised estimates, all are statistically significant given c.r. values $>1.96, p<.001$.

The convergent validity of the measurement model was supported. The factor loadings of the 25 individual/summated items in Table 5.11 were mostly greater than .70, which indicated high convergent validity, as the size of factor loadings is an important consideration of convergent validity (Hair et al., 2010). AVE estimates of the nine 
constructs were good as they were greater than .60. In addition, C.R. estimates all exceeded .80 .

Table 5.11. Standardised Factor Loadings, C.R. and AVE Statistics for Convergent Validity

\begin{tabular}{|c|c|c|c|c|c|c|}
\hline Constructs & Items & Loadings & Com.* & c.r. ${ }^{* *}$ & AVE & C.R. \\
\hline Emotional appeal (EAp) & EAp1 & 1.00 & 1.00 & 23.02 & 1.00 & 1.00 \\
\hline \multirow{3}{*}{ Emotional arousal (EAr) } & EAr1 & .87 & .753 & & \multirow{3}{*}{.83} & \multirow{3}{*}{.94} \\
\hline & EAr2 & .96 & .925 & 23.46 & & \\
\hline & EAr3 & .91 & .823 & 21.29 & & \\
\hline Coping response (CR) & CR1 & 1.00 & 1.00 & 23.02 & 1.00 & 1.00 \\
\hline \multirow{3}{*}{ Message compliance (MC) } & $M C 1^{* * *}$ & - & - & & \multirow{3}{*}{.76} & \multirow{3}{*}{.86} \\
\hline & MC2 & .98 & .956 & & & \\
\hline & MC3 & .75 & .558 & 9.04 & & \\
\hline \multirow{3}{*}{$\begin{array}{l}\text { Independent self-construal } \\
\text { (IndSC) }\end{array}$} & IndSC1 & .78 & .604 & & \multirow{3}{*}{.62} & \multirow{3}{*}{.83} \\
\hline & IndSC2 & .75 & .561 & 11.59 & & \\
\hline & IndSC3 & .83 & .684 & 12.37 & & \\
\hline \multirow{3}{*}{$\begin{array}{l}\text { Interdependent self- } \\
\text { construal (IntSC) }\end{array}$} & IntSC1 & .89 & .792 & & \multirow{3}{*}{.70} & \multirow{3}{*}{.87} \\
\hline & IntSC2 & .91 & .828 & 18.65 & & \\
\hline & IntSC3 & .69 & .479 & 12.98 & & \\
\hline \multirow{3}{*}{ Promotion focus (ProRF) } & ProRF1 & .90 & .815 & & \multirow{3}{*}{.77} & \multirow{3}{*}{.91} \\
\hline & ProRF2 & .82 & .676 & 17.84 & & \\
\hline & ProRF3 & .91 & .832 & 21.19 & & \\
\hline \multirow{3}{*}{ Prevention focus (PreRF) } & PreRF1 & .81 & .650 & & \multirow{3}{*}{.71} & \multirow{3}{*}{.88} \\
\hline & PreRF2 & .84 & .704 & 14.65 & & \\
\hline & PreRF3 & .88 & .767 & 15.06 & & \\
\hline \multirow{6}{*}{$\begin{array}{l}\text { Personal cultural orientation } \\
\text { (PCO) }\end{array}$} & PCo1 & .78 & .604 & & \multirow{6}{*}{.58} & \multirow{6}{*}{.89} \\
\hline & PCo2 & .70 & .494 & 11.55 & & \\
\hline & $\mathrm{PCo3}$ & .81 & .651 & 13.29 & & \\
\hline & PCo4 & .82 & .666 & 13.47 & & \\
\hline & PCo5 & .66 & .437 & 10.65 & & \\
\hline & PCo6 & .78 & .605 & 12.89 & & \\
\hline
\end{tabular}

* Com.: communality; $* * p<.001 ; * * *$ Item removed from final scale. 
Next, the Fornell-Larcker criterion and cross-loadings were used to evaluate discriminant validity. All the square root of AVE estimates in Table 5.12 were larger than the corresponding inter-construct correlation estimates. In addition, all bivariate correlations were less than .60 .

Table 5.12. Discriminant Validity: Fornell-Larcker Criterion

\begin{tabular}{lccccccccc}
\hline Constructs & $\mathbf{1}$ & $\mathbf{2}$ & $\mathbf{3}$ & $\mathbf{4}$ & $\mathbf{5}$ & $\mathbf{6}$ & $\mathbf{7}$ & $\mathbf{8}$ & $\mathbf{9}$ \\
\hline 1. Emotional appeal & $\mathbf{1 . 0 0}$ & & & & & & & & \\
2. Emotional arousal & .56 & .91 & & & & & & \\
3. Coping response & .40 & .33 & $\mathbf{1 . 0 0}$ & & & & & & \\
4. Message compliance & .37 & .39 & .39 & .87 & & & & & \\
5. Independent self-construal & .06 & .10 & .08 & .10 & .78 & & & & \\
6. Interdependent self-construal & .24 & .17 & .11 & .11 & .36 & .84 & & & \\
7. Promotion focus & .13 & .05 & .08 & .06 & .55 & .43 & .88 & & \\
8. Prevention focus & .19 & .16 & .16 & .13 & -.10 & .26 & .09 & .84 & \\
9. Personal cultural orientation & .29 & .39 & .17 & .29 & .10 & .48 & .00 & .33 & $\mathbf{. 7 6}$ \\
\hline
\end{tabular}

Square root of AVEs in the diagonal; Implied correlations for each construct in the model in the lower half of the table.

Lastly, the HTMT ratio using $H T M T_{.85}$ criterion was examined to further check for discriminant validity. All HTMT values in Table 5.13 were below the criterion $(<.85)$. Therefore, the discriminant validity of all constructs is met.

Table 5.13. Discriminant Validity: HTMT Ratio

\begin{tabular}{lccccccccc}
\hline Constructs & $\mathbf{1}^{*}$ & $\mathbf{2}$ & $\mathbf{3}^{\boldsymbol{*}}$ & $\mathbf{4}$ & $\mathbf{5}$ & $\mathbf{6}$ & $\mathbf{7}$ & $\mathbf{8}$ & $\mathbf{9}$ \\
\hline 1. Emotional appeal & ${ }^{*}$ & & & & & & & & \\
2. Emotional arousal & - & & & & & & & \\
3. Coping response & - & - & & & & & & & \\
4. Message compliance & - & .39 & - & & & & & & \\
5. Independent self-construal & - & .13 & - & .14 & & & & & \\
6. Interdependent self-construal & - & .19 & - & .14 & .35 & & & & \\
7. Promotion focus & - & .04 & - & .08 & .56 & .39 & & & \\
8. Prevention focus & - & .17 & - & .12 & -.07 & .35 & .05 & & \\
9. Personal cultural orientation & - & .38 & - & .28 & .12 & .58 & .00 & .33 & \\
\hline
\end{tabular}

\footnotetext{
* HTMT ratio is not applicable to two single-item constructs (EAp, CR).
} 
In all, the results of the goodness-of-fit, reliability, convergent validity, and discriminant validity indicate that the fit of the overall measurement model is deemed acceptable.

\subsection{Structural Model}

\subsubsection{Testing Hypotheses}

There are two different types of analyses used in this study to test hypotheses: path analysis and multigroup analysis.

\subsubsection{Path analysis}

Path analysis is an extension of multiple regression analysis in that it can accommodate a series of dependence relationships simultaneously (Streiner, 2005). It concurrently allows for variables to be independent with respect to some variables and dependent with respect to others. For example, guilt/shame appeals create guilt/shame arousals, and then guilt/shame arousals create message compliance, therefore guilt/shame arousals are both independent and dependent variables in the same theory.

In many cases, SEM - a more sophisticated technique - has replaced path analysis. In SEM, looking at unobserved (latent) rather than observed variables, the structural model defines relationships among the latent variables and observed variables. Accordingly, the structural model specifies "the manner by which particular latent variables directly or indirectly influence (e.g., 'cause') changes in the values of certain other latent variables in the model" (Byrne, 2010, p. 13).

\subsubsection{Multigroup analysis}

In SEM, it is possible to examine more than one group at a time (structural model testing) and compare whether or not the model fits equally well across groups (measurement model testing). Accordingly, the model can be extended in two ways: (i) multiple-group analysis (measurement invariance) and (ii) the inclusion of means (group comparison, i.e., moderation effect). These extensions are "especially powerful when they are applied simultaneously, because they offer an alternative to analysis of (co)variance for testing multiple groups for different means" (Hox \& Bechger, 1998, p. 13). 


\subsubsection{Path Analysis Evaluation}

The evaluation of structural model testing may begin once the measurement model has attained an acceptable fit. The initial structural model, constructed based on the existing literature, theory and conceptualisation, contains all paths between constructs (Koufteros, 1999). In SEM, the terms 'exogenous' and 'endogenous' variables are used instead of independent and dependent variables. Correspondingly, each path from an exogenous to endogenous variable represents an explicit research hypothesis to be tested. In this study, there are three hypotheses (four paths) to be examined.

In the structural model, as presented in Figures 5.5 and 5.6, GAp/SAp is treated as the exogenous variables, and GAr/SAr, CR and MC as the endogenous variables. Structural equation parameters include path coefficients $(\beta)$, significance values $(p)$, and variance explained $\left(R^{2}\right)$ for each endogenous variable (see more in Table 5.14). With statistical significance $(p<.05)$, the path strength is evaluated based on standardised $\beta$ that range from -1 to +1 (Hoe, 2008). According to Cohen (1988, pp. 79-81), a correlation of |.10| represents a 'small', |.30| a 'medium' and $|.50|$ a 'large' effect size $(\beta)$.

\subsubsection{Direct effects}

Table 5.14. Structural Model Path Coefficients and Significance Level

\begin{tabular}{lcc}
\hline Paths & Path coefficient $(\boldsymbol{\beta})$ & $p$-value \\
\hline Guilt model & & \\
Guilt appeal $\rightarrow$ Guilt arousal & .546 & $* * *$ \\
Guilt arousal $\rightarrow$ Coping response & .313 & $* * *$ \\
Guilt arousal $\rightarrow$ Message compliance & .360 & $* * *$ \\
Coping response $\rightarrow$ Message compliance & .275 & $* * *$ \\
\hline Shame model & & $* * *$ \\
Shame appeal $\rightarrow$ Shame arousal & .616 & $* * *$ \\
Shame arousal $\rightarrow$ Coping response & .380 & $* * *$ \\
Shame arousal $\rightarrow$ Message compliance & .242 & \\
Coping response $\rightarrow$ Message compliance & .306 & \\
\hline
\end{tabular}

$* * p<.01, * * * p<.001$. 


\section{Guilt}

The results from the analysis of the structural model's goodness-of-fit with $\chi^{2} / d f=1.98$, $\mathrm{TLI}=.97, \mathrm{CFI}=.98, \mathrm{sRMR}=.067, \mathrm{RMSEA}=.087(.032-.138)$ indicate that the model had a good fit. The key fit index RMSEA value is above the suggested cut-off of .70 but still below the moderate level of .10 (Hu \& Bentler, 1999) is deemed acceptable.

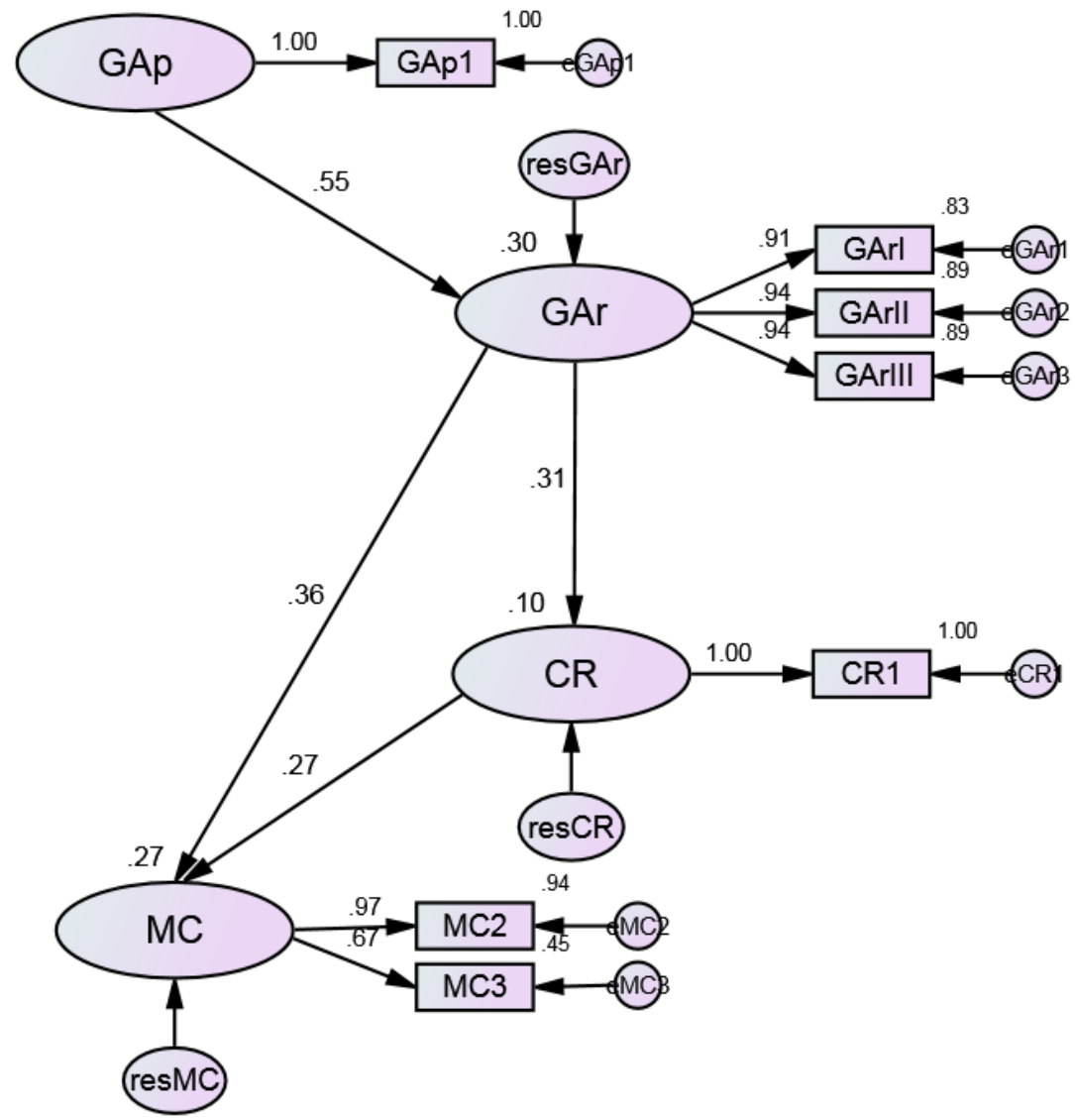

Figure 5.5. Structural Model for Guilt

The results of the path analysis, as shown in Figure 5.5, indicate that four paths had a significant relationship between the constructs (all $p$ s $<.001$ ). GAp explains $30 \%$ of the variance of GAr and 10\% of the variance of $\mathrm{CR}$ is explained by GAr. $27 \%$ of the variance of MC is explained by GAr and CR.

GAp has a strong positive impact on $\operatorname{GAr}(\beta=.546, p<.001)$. GAr has a medium positive impact on $\mathrm{CR}(\beta=.313, p<.001)$ as well as on $\mathrm{MC}(\beta=.360, p<.001)$. However, $\mathrm{CR}$ has a small positive impact on $\operatorname{MC}(\beta=.275, p<.001)$. 


\section{Shame}

The results from the analysis of the structural model's goodness-of-fit, with $\chi^{2} / d f=1.84$, $\mathrm{TLI}=.97, \mathrm{CFI}=.98, \mathrm{sRMR}=.044, \mathrm{RMSEA}=.073(.000-.125)$, indicate that the model had a good fit.

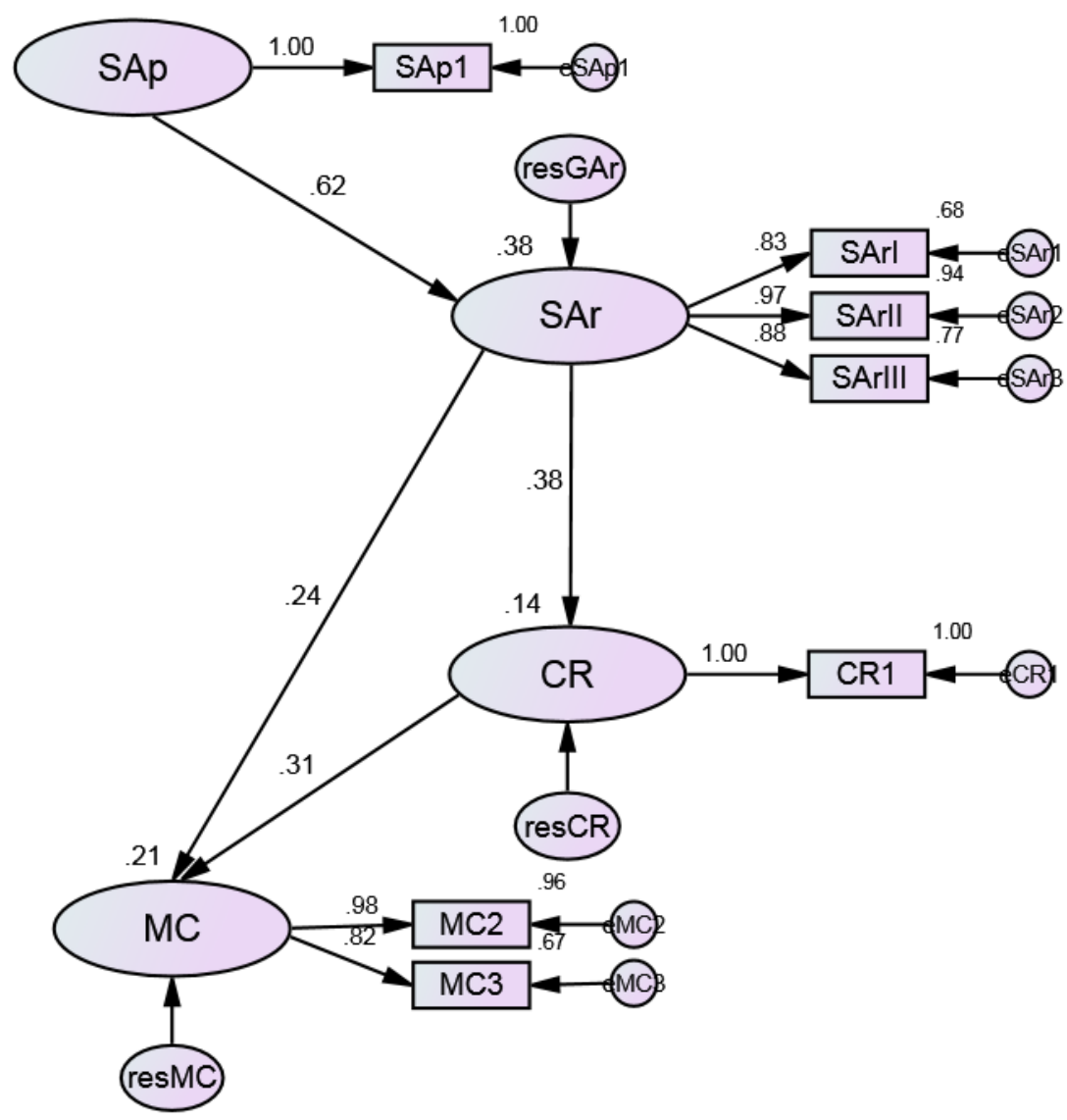

Figure 5.6. Structural Model for Shame

The results of the path analysis, displayed in Figure 5.6, show that four paths had a significant relationship between the constructs (all $p$ s $<.01$ ). SAp explains 38\% of the variance of SAr and 14\% of the variance of CR is explained by SAr. $21 \%$ of the variance of MC is explained by SAr and CR.

SAp has a strong positive impact on $\operatorname{SAr}(\beta=.616, p<.001)$. SAr has a medium positive impact on $\mathrm{CR}(\beta=.380, p<.001)$ as well as has a small positive impact on $\mathrm{MC}(\beta=.242$, $p=.006)$. However, $\mathrm{CR}$ has a medium positive impact on $\mathrm{MC}(\beta=.306, p<.001)$.

Therefore, $\mathrm{H} 1$ and $\mathrm{H} 2$ are accepted. That is, guilt or shame appeals have an impact on respective guilt or shame arousals; specifically, a high level of emotional appeal is 
associated with a high level of emotional arousal (H1a, b). Guilt or shame arousals have an impact on message compliance; specifically, a high level of emotional arousal is associated with a high level of message compliance (H2a, b).

\subsubsection{Examination of indirect effects}

Apart from the direct effects discussed above, there are several indirect effects presented in the model of guilt and shame. To investigate the model results, a fuller bootstrapping was conducted to determine indirect and total effects (Preacher \& Hayes, 2008b).

\section{Use of bootstrapping}

Bootstrapping is considered one of the better methods for estimating and testing hypotheses regarding mediation (Preacher \& Hayes, 2008b; Shrout \& Bolger, 2002). Actually, it has been a highly recommended method in recent years and seen as a logical way to quantify an indirect effect (Preacher \& Hayes, 2008a; Shrout \& Bolger, 2002).

If the variables have measurement errors, the significance of the indirect effect is likely to be underestimated. Bootstrapping can assess the stability of parameter estimates. MacKinnon and co-authors' $(2002 ; 2004)$ simulation results of the different mediation testing methods suggest that bootstrapping the indirect effect is superior to the traditional methods such as the causal steps methods, both in terms of power and Type I error rates. In addition, an increasing number of statisticians are advocating a move away from traditional statistical procedures that rely on assumptions, particularly when they are unrealistic (Efron \& Tibshirani, 1994; Preacher \& Hayes, 2008b). Using bootstrapping for testing mediation does not impose the assumption of normality of the sampling distribution and, as a result, it produces a more accurate inference (Preacher \& Leonardelli, 2010). It is preferred over methods that assume symmetry or normality of the sampling distribution of the indirect effect (Preacher \& Hayes, 2008b).

\section{Bootstrapping procedure}

Bootstrapping is an appropriate way to control and check the stability of the results. It provides stable inference in regards to the specific effects. To be more specific, it provides the most powerful and reasonable method of obtaining confidence limits for specific indirect effects under most conditions, in particular, bias-corrected (BC) bootstrapping, and is thus a highly recommended method (Preacher \& Hayes, 2008b; 
Williams \& MacKinnon, 2008). The BC bootstrap contains a correction for the bias created by the central tendency of the estimate. Bias-corrected confidence intervals (BCCI) are considered to yield more accurate values than percentile confidence intervals, as they correct for skew in the population, and are therefore recommended in testing for mediation (Fritz \& MacKinnon, 2007).

The indirect effect of GAr/SAr on MC was bootstrapped using the AMOS bootstrapping function. There is no consensus as to how many bootstrap samples should be generated, except that more is better. Preacher and Hayes (2008a) suggest at least 1,000. In the end, 2,000 re-samples were used for final reporting. A bootstrap was performed using the ML estimator, and BCCI for each of the parameter bootstrap estimates were set to the $95 \%$ level $(\alpha=.05)$.

\subsubsection{Indirect effects}

Establishing the significance of an initial direct effect is a pre-condition for testing any mediation effect (Baron \& Kenny, 1986). In other words, GAr/ SAr must first be shown to have a significant direct effect on MC without CR. As shown in Table 5.15, these direct effects for guilt and shame are both significant at the .001 level.

Table 5.15. Direct and Indirect Effects for Guilt/Shame (bootstrapping $\boldsymbol{n}=\mathbf{2 , 0 0 0 \text { ; }}$

$$
\alpha=.05)
$$

\begin{tabular}{lcccc}
\hline Paths & $\begin{array}{c}\text { Direct effects } \\
(\boldsymbol{\beta}) \text { without } \mathrm{CR}\end{array}$ & $\begin{array}{c}\text { Direct effects } \\
(\boldsymbol{\beta}) \text { with CR }\end{array}$ & $\begin{array}{c}\text { Indirect } \\
\text { effects }(\boldsymbol{\beta})\end{array}$ & $\begin{array}{c}\text { Total } \\
\text { effects }(\boldsymbol{\beta})\end{array}$ \\
\hline $\begin{array}{l}\text { Guilt model } \\
\text { Partial mediation: GAr } \rightarrow \mathrm{CR} \rightarrow \mathrm{MC}\end{array}$ & $.429^{* * *}$ & $.360^{* *}$ & $\begin{array}{c}.086^{* *} \\
(.027-.184)\end{array}$ & $.446^{* *}$ \\
\hline Shame model & & & $.116^{* * *}$ & \\
Partial mediation: $\mathrm{SAr} \rightarrow \mathrm{CR} \rightarrow \mathrm{MC}$ & $.358^{* * *}$ & $.242^{*}$ & $(.052-.216)$ & $.358^{* *}$ \\
\hline
\end{tabular}

$* p<.05, * * p<.01, * * * p<.001$; Lower bound (LB) and upper bound (UB) of estimates in parentheses.

The results of the bootstrap revealed significant indirect effects between GAr/SAr and MC through CR serving as a mediator factor. Indirect effects are considered significant when the BCCI does not include zero (Preacher \& Hayes, 2008b). The indirect effect size of GAr on MC is $\beta=.086$ ( $\mathrm{LB}=.027, \mathrm{UB}=.184, p=.002$ ); $\mathrm{SAr}$ on $\mathrm{MC}$ is $\beta=.116$ (LB $=.052, \mathrm{UB}=.216, p=.001)$ indicating that GAr/SAr has a small effect at Cohen's (1988) 
standard on MC in part through CR. Furthermore, the direct effects (with CR) of these paths for both guilt $(\beta=.360, p=.002)$ and shame $(\beta=.242, p=.016)$ are significant (see Table 5.15). Thus, $\mathrm{CR}$ had a partial mediation effect on the relationship between $\mathrm{GAr} / \mathrm{SAr}$ and MC (Hair et al., 2010).

\section{Guilt}

In the structural model, all four of the hypothesised paths (GAp $\rightarrow$ GAr, GAr $\rightarrow$ CR, GAr $\rightarrow \mathrm{MC}$, and $\mathrm{CR} \rightarrow \mathrm{MC}$ ) are significant, at least at the .001 level. The model (refer to Table 5.14 and Figure 5.5) demonstrates that GAp significantly and positively influences GAr. In turn, GAr significantly and positively influences $\mathrm{CR}$ as well as MC. It also demonstrated that $\mathrm{CR}$ significantly and positively influences MC. This finding is consistent with the underlying theory and conceptualisation of the original model. The original model hypothesised that guilt contributed positively to the relationship between coping and compliance with the emotional appeal. The result of the specified model provides empirical evidence that guilt arousal contributes to coping response, as discussed in Section 5.6.2.4 below, indirectly to message compliance.

\section{Shame}

As with guilt, in the structural model of shame, all four of the hypothesised paths (SAp $\rightarrow$ $\mathrm{SAr}, \mathrm{SAr} \rightarrow \mathrm{CR}, \mathrm{SAr} \rightarrow \mathrm{MC}$, and $\mathrm{CR} \rightarrow \mathrm{MC}$ ) are significant, at least at the .01 level. The model (refer to Table 5.14 and Figure 5.6) demonstrates that SAp significantly and positively influences SAr. In turn, SAr significantly and positively influences CR as well as MC. It also demonstrated that CR significantly and positively influences MC. This finding is also consistent with the underlying theory and conceptualisation of the original model of shame. The original model hypothesised that shame contributed positively to the relationship between coping and compliance with the emotional appeal. The result of the specified model provides empirical evidence that shame arousal contributes to coping response, as discussed in Section 5.6.2.4 below, indirectly to message compliance.

\subsubsection{Total effects}

The total effects consider not only the direct effects among the constructs, but also the indirect effects on the dependent (endogenous) variables (Cool, Dierickx, \& Jemison, 1989). The indirect effect is manifest in the model and relevant in the evaluation, 
interpretation and understanding of the total impact of one construct on another within the model of guilt/shame appeal-compliance.

\section{Guilt}

In this case, even though GAr has a direct effect on MC, GAr does have a positive and significant indirect effect on MC through CR. Consider the effect of GAr on MC. The direct effect is .360 (the path coefficient from GAr $\rightarrow$ MC). The indirect effect, through $\mathrm{CR}$, is $.086^{8}$. The total effect is the sum of direct and indirect effects, $(.360+.086)=.446$ (Kline, 2016) (see Table 5.15).

\section{Shame}

As with the guilt model, even though SAr has a direct effect on MC, SAr does have a positive and significant indirect effect on MC through CR. Consider the effect of SAr on MC. The direct effect is .242 (the path coefficient from SAr $\rightarrow$ MC). The indirect effect, through CR, is $.116^{9}$. The total effect is the sum of direct and indirect effects, $(.242+$ $.116)=.358($ Kline, 2016$)($ see Table 5.15) .

The results of the total effects analysis demonstrate that guilt/shame emotional arousal positively and significantly influences compliance with the emotional appeal, through coping response. The results also show that the total effect of emotional arousal on message compliance is much stronger than the direct effect of emotional arousal on message compliance. This supports the study's overall thesis that emotional arousal influences coping response and indirectly inhibits message compliance.

Therefore, $\mathrm{H} 3$ is accepted: Coping responses partially mediate the relationship between guilt or shame arousal and message compliance.

\subsubsection{Control variables}

Control variables are potentially confounding factors that can affect some of the constructs of interest. The effect of potential confounding variables can be handled

\footnotetext{
${ }^{8}$ From Table 5.14, the indirect effect is computed as the product of the path coefficient from guilt arousal to coping response and the path coefficient from coping response to message compliance, $(.313)(.275)=.086$.

${ }^{9}$ From Table 5.14, the indirect effect is computed as the product of the path coefficient from shame arousal to coping response and the path coefficient from coping response to message compliance, $(.380)(.306)=$ .116 .
} 
adequately by incorporating them into an appropriate regression analysis (Gaskin, 2012b; MacKinnon, Krull, \& Lockwood, 2000).

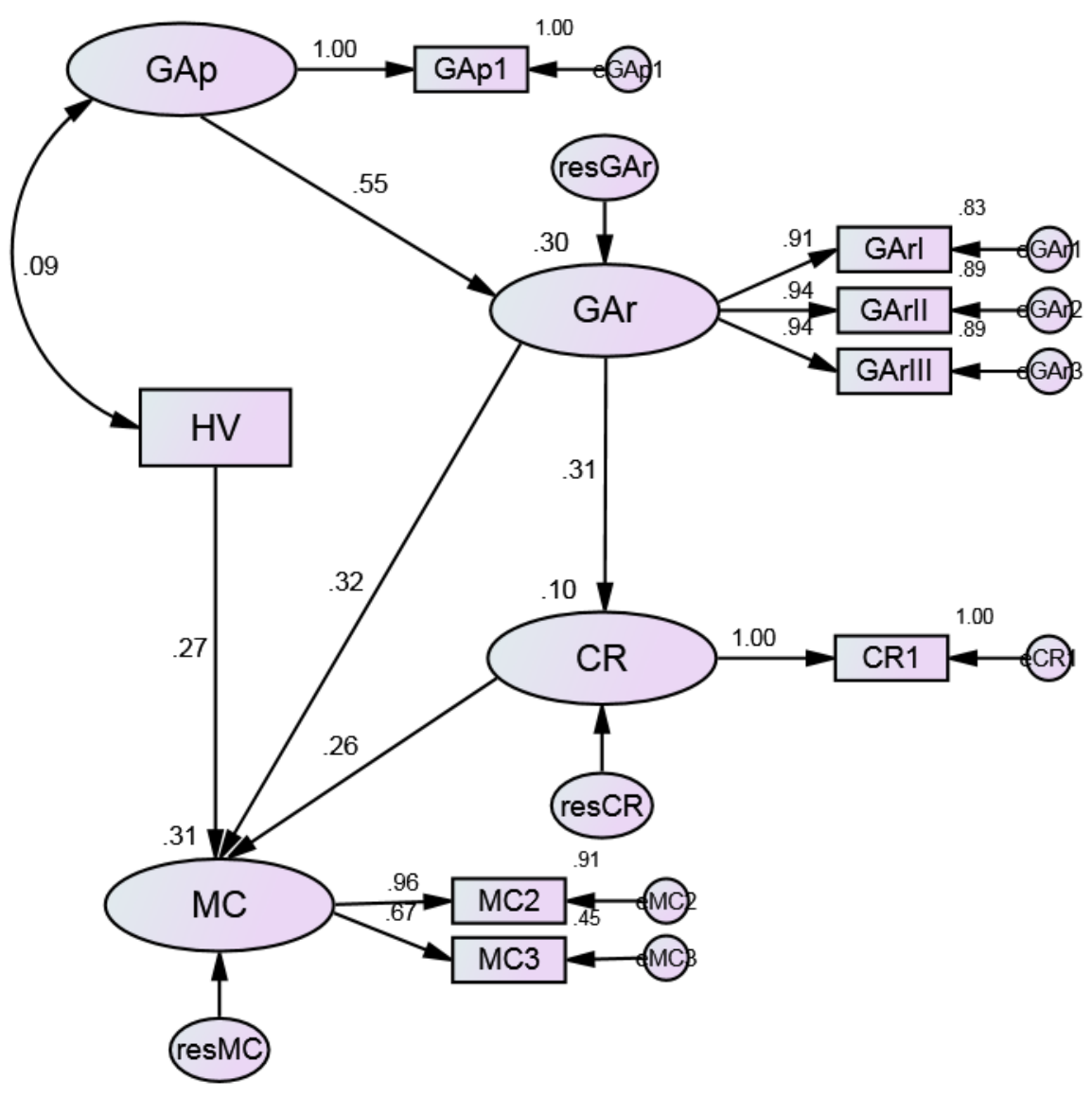

Figure 5.7. Structural Model for Guilt with Control

Path analysis was run to assess the effects of the independent variables (GAr/SAr and $\mathrm{CR}$ ) on the dependent variable (MC), while controlling for health message exposure (HME), health message involvement (HMI), and health value (HV). Three paths (HME $\rightarrow \mathrm{MC}, \mathrm{HMI} \rightarrow \mathrm{MC}$, and HV $\rightarrow \mathrm{MC}$ for both guilt and shame, see Table 5.16) show no significant relationship between the constructs ( $p$ s $>.05$ ), except for the path $\mathrm{HV} \rightarrow \mathrm{MC}$ for guilt $(\beta=.268, p<.001)$. The paths that were specified but not significant were dropped (Byrne, 2010) and the model then re-estimated (Kenny, 2014; Schumacker \& Lomax, 2004).

The path coefficients in Figure 5.7 are from the trimmed model. All path coefficients were statistically significant $(p s<.01)$. A comparison of the two sets of path coefficients (see Table 5.16, numbers in parentheses represent $\beta$ values without control variables) suggested that controlling for HV had very little effect on the strength of the relationship 
between these sets of two variables ( $\mathrm{GAr} \rightarrow \mathrm{MC}, \mathrm{CR} \rightarrow \mathrm{MC}$ ). It is also noted that a higher $R^{2}$ (for MC 307 compared to .267) but with increasingly smaller $\beta$ s (for GAr $\rightarrow$ MC .323 compared to .360, for CR $\rightarrow$ MC .268 compared to .275) as a result of the control variable $\mathrm{HV}$ was added into the analysis.

Table 5.16. Structural Model Path Coefficients and Significance Level with Control

\begin{tabular}{lcc}
\hline Paths & Path coefficient $(\boldsymbol{\beta})$ & $\boldsymbol{p}$-value \\
\hline Guilt model & & \\
Health message exposure $\rightarrow$ Message compliance & - & $\mathrm{ns}$ \\
Health message involvement $\rightarrow$ Message compliance & - & $\mathrm{ns}$ \\
Health value $\rightarrow$ Message compliance & .268 & $* * *$ \\
Guilt arousal $\rightarrow$ Message compliance & $.323(.360)$ & $* *$ \\
Coping response $\rightarrow$ Message compliance & $.268(.275)$ & $* * *$ \\
\hline Shame model & & $\mathrm{ns}$ \\
Health message exposure $\rightarrow$ Message compliance & - & $\mathrm{ns}$ \\
Health message involvement $\rightarrow$ Message compliance & - & $\mathrm{ns}$ \\
Health value $\rightarrow$ Message compliance & - & \\
\hline
\end{tabular}

ns: no significance, $* * p<.01, * * * p<.001$; numbers in parentheses represent $\beta$ values without control variables.

\subsubsection{Multigroup Analysis}

\subsubsection{Measurement model invariance testing}

The study aims to compare different dominant self-construal (IndSC and IntSC), dominant regulatory focus (ProRF and PreRF) and personal cultural orientation (IndPCO and ColPCO) on the constructs of interest (i.e., guilt/shame arousals, message compliance). Prior to comparing these groups on a construct, a critical concern raised is whether or not components of the measurement model are equivalent/invariant across particular groups of interest to assure that the measurement model is group-invariant. Basically, this concern is justified through testing for the factorial equivalence/invariance of the construct across different samples. The invariance of a measurement model across different groups is called measurement invariance (or factorial invariance). Several levels of factorial invariance can be differentiated (Meredith, 1993; Zumbo, Sireci, \& Hambleton, 2003). In the framework of CFA, testing for factorial invariance should include the configural, metric and scalar invariance (Comşa, 2010; Hair et al., 2010). 


\section{Configural invariance}

The lowest level of factorial invariance is referred to as configural invariance. At this level, factorial invariance requires that the number of factors and factor-loading pattern should be the same across different groups. The model configuration (the pattern of fixed and non-fixed model parameters) being invariant across groups is seen as configural invariance (Dimitrov, 2010). As such, the measurement model imposed no equality constraints on any of the parameters and is judged based on "the adequacy of the goodness-of-fit statistics only" (Byrne, 2010, p. 239).

\section{Metric invariance}

The next level of factorial invariance is metric invariance. Metric invariance is tested by imposing an equality constraint on the factor pattern coefficients (loadings) across the compared groups. In the measurement, the loadings carry the information about the relationship between the observed items and the latent variable. If metric invariance is obtained, it can assume that respondents in the different groups interpret the construct items in the same way. Even though this metric invariance does not ensure that the construct is measured in the same way (scalar invariance), if established, comparisons on covariances or unstandardised regression coefficients can be meaningful across groups (Hair et al., 2010; Steenkamp \& Baumgartner, 1998). In this case, differences in observed items are a result of differences in the latent construct across groups.

\section{Scalar invariance}

The third level of factorial invariance is called scalar invariance. Scalar invariance is tested by imposing equality constraints on not only the factor loadings but also the item intercepts across groups. If the model fit does not prove to be significantly worse in comparison with the metric invariance model, this would mean that scalar invariance is obtained. If scalar invariance is obtained, it can assume that people in the different groups respond to the construct in the same way. At this level, as the same construct is measured in the same way, comparisons of latent means between the groups are meaningful (Davidov \& Depner, 2011). This is because differences in the latent construct means indicate differences in the construct item scores, not differences in factor loadings or item intercepts. 
In sum, comparisons of the latent construct means are made when scalar invariance is established. Note that although full invariance is always stronger, partial invariance is accepted (Hair et al., 2010). Partial invariance is obtained when the parameters of at least two items per construct are invariant across groups (i.e., partial metric invariance loadings and partial scalar invariance loadings plus intercepts).

\subsubsection{Measurement model evaluation}

Group models were specified based on the three-stage process and then estimated. In this study, the groups were of almost equal size (see Section 5.5.2). With the defined groups (i.e., self-construal, regulatory focus, personal cultural orientation), the invariance testing process can begin.

\section{Across Self-construal}

\section{Guilt}

Table 5.17 contains the model fit statistics for each model and the $\chi^{2}$ difference test for each model comparison.

Table 5.17. Measurement Invariance Tests for Guilt - Self-Construal

\begin{tabular}{|c|c|c|c|c|c|c|c|c|c|}
\hline \multirow{2}{*}{ Model tested } & \multicolumn{6}{|c|}{ Model fit measures } & \multicolumn{3}{|c|}{ Model differences } \\
\hline & $x^{2}$ & $d f$ & $p$ & $T L I$ & $C F I$ & RMSEA & $\Delta X^{2}$ & $\Delta d f$ & $p$ \\
\hline \multicolumn{10}{|l|}{ Separate groups } \\
\hline IndSC & 13.440 & 10 & .200 & .976 & .988 & .073 & & & \\
\hline IntSC & 9.099 & 10 & .523 & 1.007 & 1.000 & .000 & & & \\
\hline Configural invariance & 22.539 & 20 & .312 & .991 & .996 & .031 & & & \\
\hline Metric invariance & 26.560 & 23 & .275 & .989 & .994 & .035 & 4.020 & 3 & .259 \\
\hline Scalar invariance & 35.093 & 28 & .167 & .982 & .988 & .044 & 8.533 & 5 & .129 \\
\hline
\end{tabular}

In the first stage of configural invariance, the separate models for IndSC and IntSC both exhibit acceptable levels of model fit, as does the combined multisample confirmatory factors analysis (MCFA) model: $\chi^{2} / d f=1.13$; TLI $=.99 ; \mathrm{CFI}=1.00 ;$ RMSEA $=.031$. Note that the MCFA $\chi^{2}$ is equal to the sum of the two groups - indicating the configural invariance (Hair et al., 2010). 
The next test, metric invariance, involves constraining each matching loading to be equal across the groups. We can see that the $\chi^{2}$ difference test was nonsignificant $\left(\Delta \chi^{2}(3)=\right.$ $4.020, p=.259$ ). Thus, the two models (IndSC and IntSC) exhibit full metric invariance.

The last stage is to test for scalar invariance. The $\chi^{2}$ difference test was nonsignificant $\left(\Delta \chi^{2}(5)=8.533, p=.129\right)$, indicating that full scalar variance is supported. As a result, the scalar invariance model is accepted (Hu \& Bentler, 1999; Marsh, Hau, \& Wen, 2004).

\section{Shame}

As with guilt, in the first stage of configural invariance for shame, the separate models for IndSC and IntSC both exhibit acceptable levels of model fit: $\chi^{2} / d f=1.30 ;$ TLI $=.98 ;$ CFI $=.99 ; \mathrm{RMSEA}=.048$ (see Table 5.18). Again, the MCFA $\chi^{2}$ is equal to the sum of the two groups - indicating the configural invariances. Test for metric invariance shows that the $\chi^{2}$ difference test was nonsignificant $\left(\Delta \chi^{2}(3)=1.661, p=.646\right)$. Thus, the two models (IndSC and IntSC) exhibit full metric invariance.

Table 5.18. Measurement Invariance Tests for Shame - Self-Construal

\begin{tabular}{|c|c|c|c|c|c|c|c|c|c|}
\hline \multirow{2}{*}{ Model tested } & \multicolumn{6}{|c|}{ Model fit measures } & \multicolumn{3}{|c|}{ Model differences } \\
\hline & $x^{2}$ & $d f$ & $p$ & $T L I$ & CFI & RMSEA & $\Delta X^{2}$ & $\Delta d f$ & $p$ \\
\hline \multicolumn{10}{|l|}{ Separate groups } \\
\hline IndSC & 25.731 & 10 & .108 & .955 & .979 & .101 & & & \\
\hline IntSC & 10.356 & 10 & .410 & .998 & .999 & .021 & & & \\
\hline Configural invariance & 26.086 & 20 & .163 & .978 & .989 & .048 & & & \\
\hline Metric invariance & 27.747 & 23 & .225 & .985 & .992 & .039 & 1.661 & 3 & .646 \\
\hline Scalar invariance & 31.553 & 28 & .293 & .991 & .994 & .031 & 3.806 & 5 & .578 \\
\hline
\end{tabular}

The next stage is to test for scalar invariance. The $\chi^{2}$ difference test was nonsignificant $\left(\Delta \chi^{2}(5)=3.806, p=.578\right)$, indicating that full scalar variance is supported. As per the result, we cannot reject the scalar invariance model (Hu \& Bentler, 1999; Marsh et al., 2004).

The measurement invariance testing for both guilt and shame models met the criteria for configural invariance, full metric invariance and full scalar invariance. As a result, almost any form of group comparison can be made without concern that the differences are due to the differing measurement properties between the two self-construal groups (Hair et al., 2010). 


\section{Across Regulatory focus}

\section{Guilt}

In configural invariance testing, the configural model was found to be well-fitting in its representation of the multigroup data: $\chi^{2} / d f=1.03$; TLI $=1.00 ;$ CFI $=1.00 ;$ RMSEA $=$ .016 (see Table 5.19). This finding allows this study to proceed to the next step. Testing for metric invariance revealed that the second step of invariance testing resulted in nonsignificant $\chi^{2}$ difference $(p=.119)$. Thus, the two models for ProRF and PreRF exhibit full metric invariance.

Table 5.19. Measurement Invariance Tests for Guilt - Regulatory Focus

\begin{tabular}{|c|c|c|c|c|c|c|c|c|c|}
\hline \multirow{2}{*}{ Model tested } & \multicolumn{6}{|c|}{ Model fit measures } & \multicolumn{3}{|c|}{ Model differences } \\
\hline & $x^{2}$ & $d f$ & $p$ & $T L I$ & CFI & RMSEA & $\Delta X^{2}$ & $\Delta d f$ & $p$ \\
\hline \multicolumn{10}{|l|}{ Separate groups } \\
\hline ProRF & 6.683 & 10 & .755 & 1.018 & 1.000 & .000 & & & \\
\hline PreRF & 13.934 & 10 & .176 & .958 & .980 & .102 & & & \\
\hline Configural invariance & 20.617 & 20 & .420 & .998 & .999 & .016 & & & \\
\hline Metric invariance & 26.474 & 23 & .279 & .989 & .994 & .034 & 5.857 & 3 & .119 \\
\hline Scalar invariance & 33.703 & 28 & .211 & .985 & .990 & .040 & 7.229 & 5 & .204 \\
\hline
\end{tabular}

Again, for scalar invariance the $\chi^{2}$ difference test was nonsignificant $\left(\Delta \chi^{2}(5)=7.229, p=\right.$ .204). As per the result, the scalar invariance model is accepted and comparisons between construct means are possible.

\section{Shame}

In configural invariance testing, the configural model was found to be well-fitting in its representation of the multigroup data: $\chi^{2} / d f=1.29$; TLI $=.98 ; \mathrm{CFI}=.99 ; \mathrm{RMSEA}=.047$ (see Table 5.20). This finding allows this study to proceed to the next step of invariance testing. The result shows nonsignificant $\chi^{2}$ difference test $(p=.142)$. Thus, the two models for ProRF and PreRF exhibit full metric invariance.

The scalar invariance model testing involved constraining the intercepts for each observed variable to be equal across the groups. Again, the $\chi^{2}$ difference test was nonsignificant $\left(\Delta \chi^{2}(5)=10.454, p=.063\right)$. As per the result, we cannot reject the scalar invariance model. 
Table 5.20. Measurement Invariance Tests for Shame - Regulatory Focus

\begin{tabular}{|c|c|c|c|c|c|c|c|c|c|}
\hline \multirow{2}{*}{ Model tested } & \multicolumn{6}{|c|}{ Model fit measures } & \multicolumn{3}{|c|}{ Model differences } \\
\hline & $x^{2}$ & $d f$ & $p$ & $T L I$ & CFI & RMSEA & $\Delta X^{2}$ & $\Delta d f$ & $p$ \\
\hline \multicolumn{10}{|l|}{ Separate groups } \\
\hline ProRF & 12.912 & 10 & .229 & .985 & .993 & .054 & & & \\
\hline PreRF & 12.951 & 10 & .226 & .960 & .981 & .095 & & & \\
\hline Configural invariance & 25.863 & 20 & .170 & .979 & .990 & .047 & & & \\
\hline Metric invariance & 31.314 & 23 & .115 & .974 & .9486 & .052 & 5.450 & 3 & .142 \\
\hline Scalar invariance & 41.767 & 28 & .046 & .964 & .976 & .061 & 10.454 & 5 & .063 \\
\hline
\end{tabular}

The above results of the metric and scalar invariance tests for both guilt and shame models allowed accepting the hypothesis of equal intercepts and equal regression weights in the measurement model, and the mean comparisons can be carried out with confidence as well.

\section{Across Personal cultural orientation}

\section{Guilt}

In configural invariance testing, the configural model was found to be well-fitting in its representation of the multigroup data: $\chi^{2} / d f=.83 ; \mathrm{TLI}=1.01 ; \mathrm{CFI}=1.00 ; \mathrm{RMSEA}=$ .000 (see Table 5.21). This finding allows this study to proceed to the next step.

Testing for metric invariance revealed that the second step of invariance testing resulted in significant $\chi^{2}$ difference $(p=.101)$. Thus, the two models for IndPCO and ColPCO exhibit full metric invariance.

Table 5.21. Measurement Invariance Tests for Guilt - Personal Cultural Orientation

\begin{tabular}{|c|c|c|c|c|c|c|c|c|c|}
\hline \multirow{2}{*}{ Model tested } & \multicolumn{6}{|c|}{ Model fit measures } & \multicolumn{3}{|c|}{ Model differences } \\
\hline & $x^{2}$ & $d f$ & $p$ & $T L I$ & CFI & RMSEA & $\Delta X^{2}$ & $\Delta d f$ & $p$ \\
\hline \multicolumn{10}{|l|}{ Separate groups } \\
\hline IndPCO & 7.591 & 10 & .669 & 1.022 & 1.000 & .000 & & & \\
\hline ColPCO & 9.062 & 10 & .526 & 1.007 & 1.000 & .000 & & & \\
\hline Configural invariance & 16.653 & 20 & .675 & 1.013 & 1.000 & .000 & & & \\
\hline Metric invariance & 22.874 & 23 & .468 & 1.000 & 1.000 & .000 & 6.221 & 3 & .101 \\
\hline Scalar invariance & 37.787 & 28 & .102 & .972 & .981 & .052 & 14.914 & 5 & .011 \\
\hline
\end{tabular}


The next stage is to test for scalar invariance. Here, the $\chi^{2}$ difference test was significant $\left(\Delta \chi^{2}(5)=14.914, p=.011\right)$, indicating that full scalar variance is not supported. However, according to Hair et al. (2010), for partial scalar invariance, a model constraining at least two parameters per construct (i.e., GArI, GArII for GAr; MC2, MC3 for MC based on MI for the scalar invariance model examination to reduce the $\chi^{2}$ difference the most), the $\chi^{2}$ difference test was nonsignificant from the metric invariance model $\left(\Delta \chi^{2}(4)=8.346, p=\right.$ .080). Thus, partial scalar invariance is supported as well. As per the result, we cannot reject the scalar invariance model. Thus, comparisons between construct means are possible.

\section{Shame}

In configural invariance testing, the configural model was found to be well-fitting in its representation of the multigroup data: $\chi^{2} / d f=1.44$; TLI $=.97$; CFI $=.99 ;$ RMSEA $=.057$ (see Table 5.22). This finding allows this study to proceed to the next step of invariance testing. The result shows nonsignificant $\chi^{2}$ difference test $(p=.634)$. Thus, the two models for IndPCO and ColPCO exhibit full metric invariance.

The scalar invariance model testing involved constraining the intercepts for each observed variable to be equal across the groups (constrained model $\chi^{2} / d f=1.40$; TLI $=$ .97 ; CFI $=.98 ;$ RMSEA $=.054)$. Again, the $\chi^{2}$ difference test was nonsignificant $\left(\Delta \chi^{2}(5)=\right.$ $8.642, p=.124)$. As per the result, we cannot reject the scalar invariance model.

Table 5.22. Measurement Invariance Tests for Shame - Personal Cultural Orientation

\begin{tabular}{|c|c|c|c|c|c|c|c|c|c|}
\hline \multirow{2}{*}{ Model tested } & \multicolumn{6}{|c|}{ Model fit measures } & \multicolumn{3}{|c|}{ Model differences } \\
\hline & $x^{2}$ & $d f$ & $p$ & $T L I$ & CFI & RMSEA & $\Delta X^{2}$ & $\Delta d f$ & $\bar{p}$ \\
\hline \multicolumn{10}{|l|}{ Separate groups } \\
\hline IndPCO & 13.074 & 10 & .220 & .974 & .987 & .065 & & & \\
\hline ColPCO & 15.638 & 10 & .110 & .963 & .982 & .095 & & & \\
\hline Configural invariance & 28.712 & 20 & .094 & .968 & .985 & .057 & & & \\
\hline Metric invariance & 30.423 & 23 & .138 & .976 & .987 & .049 & 1.711 & 3 & .634 \\
\hline Scalar invariance & 39.065 & 28 & .080 & .971 & .980 & .054 & 8.642 & 5 & .124 \\
\hline
\end{tabular}

The above results of the metric and scalar invariance tests for both guilt and shame models allowed accepting the hypothesis of equal intercepts and equal regression weights 
in the measurement model. As the scalar invariance was supported, the mean comparisons can be carried out with confidence between the two personal cultural orientation groups.

\subsubsection{Group Comparison}

Structural model comparisons provide the test of moderation. A moderating effect occurs when a moderator variable affects the strength or direction of the relationship between the independent and dependent variables (Baron \& Kenny, 1986, p. 1174).

Group model comparisons can identify the extent of the differences either for an entire model or a specific relationship. In this study, comparisons are made for the defined relationships within the groups: dominant self-construal (IndSC versus IntSC), dominant regulatory focus (ProRF versus PreRF), and personal cultural orientation (IndPCO versus ColPCO).

With measurement invariance established, the structural model estimate is then assessed for moderation by a comparison of group models. The first group model is estimated with path estimates calculated separately for each group (unconstrained model). Then a second group model is estimated where the path estimate of interest is constrained to an equal value (e.g., Beta) between the groups. If the $\chi^{2}$ of the model with the constrained path became significantly worse than that of the unconstrained model, it can be concluded that moderation does exist (Hair et al., 2010).

\subsubsection{Moderating effect of Regulatory focus}

A multigroup analysis was performed to test $\mathrm{H} 4$. The main focus of the comparison across the two RF groups was to establish whether the causal path in the hypothesised model (GAp/SAp $\rightarrow$ GAr/SAr) differed significantly between ProRF and PreRF.

Table 5.23. Group Comparison on the Basis of Regulatory Focus

\begin{tabular}{|c|c|c|c|c|}
\hline \multirow{2}{*}{ Paths } & \multicolumn{2}{|l|}{ ProRF } & \multicolumn{2}{|l|}{ PreRF } \\
\hline & Standard coefficients & $p$-value & Standard coefficients & p-value \\
\hline $\mathrm{GAp} \rightarrow \mathrm{GAr}$ & .563 & 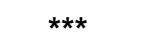 & .507 & $\star * *$ \\
\hline $\mathrm{SAp} \rightarrow \mathrm{SAr}$ & $.572^{\mathrm{a}}$ & 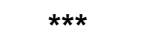 & $.770^{\mathrm{b}}$ & 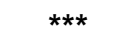 \\
\hline
\end{tabular}

*** $p<.001 ; \beta$ values in the same row with a different superscript differ marginally significantly from each other at $p<.10$. 
Table 5.24. Adjusted Means for Regulatory Focus on Guilt/Shame Arousal

\begin{tabular}{lllllr}
\hline & Regulatory focus & N & Mean & SE & \\
\hline Guilt & ProRF & 91 & $3.09^{\mathrm{a}}$ & .157 & $\mathrm{~F}(1,127)=.002$, \\
& PreRF & 39 & $3.10^{\mathrm{a}}$ & .243 & $\mathrm{p}=.963$ \\
\hline \multirow{2}{*}{ Shame } & ProRF & 102 & $2.86^{\mathrm{b}}$ & .119 & $\mathrm{~F}(1,133)=5.969$, \\
& PreRF & 34 & $3.45^{\mathrm{b}}$ & .206 & $\mathrm{p}=.016$ \\
\hline
\end{tabular}

Covariates appearing in the model are evaluated at the following values: ${ }^{\mathrm{a}} \mathrm{GAp}=3.63 ;{ }^{\mathrm{b}} \mathrm{SAp}=4.38$.

\section{Guilt}

The hypothesised model with multigroup analysis shows a good fit with the data encompassing ProRF and PreRF: $\chi^{2} / d f=1.81$; TLI $=.94$; CFI $=.97$; RMSE $=.079$.

In this multigroup analysis, the path (GAp $\rightarrow$ GAr) is significantly positive for each of these two RF (ProRF $\beta=.563, p<.001$; PreRF $\beta=.507, p<.001$ ). However, as shown in Table 5.23, the path is not significantly different across ProRF and PreRF (the $\chi^{2}$ difference test was nonsignificant, $\left.\Delta \chi_{(1)}^{2}=1.055, p=.304\right)$. As per the result, we cannot reject the constrained model. Thus, RF does not moderate the relationship between GAp and GAr. Specifically, the relationship strength between guilt appeal and guilt arousal is statistically equal strong for both promotion- and prevention-focused individuals.

For comparison of means, ANCOVA tests were used to determine there is a difference between two RF in GAr. After adjusting for GAp scores (controlling for the covariate ${ }^{10}$ ), there was no significant difference between ProRF and PreRF on GAr scores, $F(1,127)=$ $.002 p=.963$, partial eta squared $\eta^{2}=.000$ (see Table 5.24).

Thus, H4 (a) is not accepted.

\section{Shame}

The hypothesised model with multigroup analysis shows a good fit with the data encompassing ProRF and PreRF: $\chi^{2} / d f=1.49 ; \mathrm{TLI}=.96 ; \mathrm{CFI}=.98 ; \mathrm{RMSEA}=.060$.

In the multigroup analysis, the path (SAp $\rightarrow \mathrm{SAr}$ ) is significantly positive for each of these two RF (ProRF $\beta=.572, p<.001$; PreRF $\beta=.770, p<.001$ ). As shown in table 5.23 , the path is marginally significantly different across ProRF and PreRF (the $\chi^{2}$

\footnotetext{
${ }^{10}$ The effect of the covariate has been statistically removed.
} 
difference test was marginally significant, $\left.\Delta \chi_{(1)}^{2}=3.553, p=.059\right)$. This suggests that there is some evidence that the relationship between SAp and SAr is statistically stronger for PreRF than for ProRF. As per the result, we reject the constrained model. Thus, RF does moderate the relationship between SAp and SAr. Specifically, the relationship strength between shame appeal and shame arousal is statistically higher for preventionfocused than for promotion-focused individuals.

Again, ANCOVA tests were used to determine there is a difference between two RF in SAr. After adjusting for SAp scores, there was a significant difference between ProRF and PreRF on SAr, $F(1,133)=5.969, p=.016$, partial eta squared $\eta^{2}=.043$. Specifically, as shown in Table 5.24, the SAr scores for PreRF $(M=3.45, S E=.206)$ are significantly higher than for ProRF $(M=2.86, S E=.119)$.

Thus, H4 (b) is accepted.

In all, H4 is partially accepted: (a) Levels of emotional arousal elicited from a guilt appeal are not impacted by individuals' regulatory focus, whereas (b) levels of emotional arousal elicited from a shame appeal are impacted by individuals' regulatory focus. Specifically, prevention-focused individuals exhibit more shame arousal than their promotion-focused counterparts.

\subsubsection{Moderating effect of Self-construal}

To test Hypothesis 5, a multigroup analysis was performed. The main focus of the comparison across the two SC groups was to determine if the causal path in the hypothesised model (GAp/SAp $\rightarrow$ GAr/SAr) differed significantly between IndSC and IntSC.

Table 5.25. Group Comparison on the Basis of Self-Construal

\begin{tabular}{|c|c|c|c|c|}
\hline \multirow{2}{*}{ Paths } & \multicolumn{2}{|l|}{ IndSC } & \multicolumn{2}{|l|}{ IntSC } \\
\hline & Standard coefficients & $p$-value & Standard coefficients & $p$-value \\
\hline GAp $\rightarrow$ GAr & $.614^{a}$ & 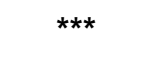 & $.502^{\mathrm{b}}$ & 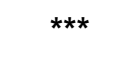 \\
\hline $\mathrm{SAp} \rightarrow \mathrm{SAr}$ & .688 & 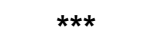 & .578 & 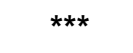 \\
\hline
\end{tabular}


Table 5.26. Adjusted Means for Self-Construal on Guilt/Shame Arousal

\begin{tabular}{lllllr}
\hline & Self-construal & N & Mean & SE & \\
\hline Guilt & IndSC & 65 & $3.36^{\mathrm{a}}$ & .181 & $\mathrm{~F}(1,127)=4.228$, \\
& IntSC & 65 & $2.83^{\mathrm{a}}$ & .181 & $\mathrm{p}=.042$ \\
\hline \multirow{2}{*}{ Shame } & IndSC & 57 & $3.07^{\mathrm{a}}$ & .162 & $\mathrm{~F}(1,133)=.219$, \\
& IntSC & 79 & $2.97^{\mathrm{a}}$ & .138 & $\mathrm{p}=.641$ \\
\hline
\end{tabular}

Covariates appearing in the model are evaluated at the following values: ${ }^{\mathrm{a}} \mathrm{GAp}=3.63 ;{ }^{\mathrm{b}} \mathrm{SAp}=4.38$.

\section{Guilt}

The hypothesised model with multigroup analysis shows a good fit with the data encompassing IndSC and IntSC: $\chi^{2} / d f=1.91 ; \mathrm{TLI}=.93 ; \mathrm{CFI}=.96 ; \mathrm{RMSEA}=.084$.

In this multigroup analysis, the path (GAp $\rightarrow$ GAr) is significantly positive for each of these two SC (IndSC $\beta=.614, p<.001 ; \operatorname{IntSC} \beta=.502, p<.001)$. As shown in Table 5.25 , the path is marginally significantly different across IndSC and IntSC (the $\chi^{2}$ difference test was marginally significant, $\left.\Delta \chi^{2}(1)=3.340, p=.068\right)$. ). This provides some support that the relationship between GAp and GAr is statistically stronger for IndSC than for IntSC. As per the result, we reject the constrained model. Thus, SC does marginally moderate the relationship between GAp and GAr. That is, the relationship strength between guilt appeal and guilt arousal is statistically higher for independent than for interdependent self-construals.

For comparison of means, ANCOVA tests were used to determine there is a difference between two SC groups in GAr. After adjusting for GAp, there was significant difference between IndSC and IntSC on GAr (regardless of type of guilt appeal), $F(1,127)=4.228$, $p=.042$; however, the effect size was small, partial eta squared $\eta^{2}=.032(3.2 \%$ variance explained). Specifically, as shown in Table 5.26, the GAr scores for IndSC ( $M=3.36, S E$ $=.181)$ are significantly higher than for $\operatorname{IntSC}(M=2.83, S E=.181)$.

Thus, H5 (a) is accepted: Independent self-construals exhibit significantly higher guilt arousal than their interdependent counterparts. 


\section{Shame}

The hypothesised model with multigroup analysis shows a good fit with the data encompassing IndSC and IntSC: $\chi^{2} / d f=1.70 ; \mathrm{TLI}=.95 ; \mathrm{CFI}=.97 ; \mathrm{RMSEA}=.073$.

In this multigroup analysis, the path (SAp $\rightarrow \mathrm{SAr}$ ) is significantly positive for each of these two SC (IndSC $\beta=.688, p<.001$; IntSC $\beta=.578, p<.001$ ). However, as shown in Table 5.25, the path is not significantly different across IndSC and IntSC (the $\chi^{2}$ difference test was nonsignificant, $\left.\Delta \chi^{2}(1)=.907, p=.341\right)$. As per the result, we cannot reject the constrained model. Thus, SC does not moderate the relationship between SAp and SAr. That is, the relationship strength between shame appeal and shame arousal is statistically equal for both independent and interdependent self-construals.

For comparison of means, again, ANCOVA tests were used to determine there is a difference between two SC groups in SAr. After adjusting for SAp, there was no significant difference between IndSC and IntSC on SAr (regardless of type of shame appeal $), F(1,133)=.219, p=.641$.

Thus, H5 (b) is not accepted: Interdependent self-construals do not exhibit significantly higher shame arousal than their independent counterparts.

\subsubsection{Interaction effect of Self-construal with Self-Referencing, Sources of Evaluation}

A two-way ANCOVA was conducted to explore the interaction effect of SC (IndSC and IntSC) and type of appeal (self-referencing: self-reference and other-reference; sources of evaluation: internal and external) on GAr/SAr after adjusting for GAp/SAp scores.

Table 5.27. Adjusted Means of Self-Construal and Type of Appeal on Guilt/Shame

\section{Arousal}

\begin{tabular}{|c|c|c|c|c|c|c|c|c|c|c|}
\hline & & & IndSC & & & & & IntSC & & \\
\hline & Self-r & rencing & Evaluat & on source & Main & Self-r $r$ & rencing & Evaluati & on source & Mo \\
\hline & Self & Other & Internal & External & & Self & Other & Internal & External & \\
\hline GAr & 3.07 & 3.39 & 3.70 & 3.02 & $3.36^{a}$ & 2.74 & 3.49 & 2.81 & 2.60 & $2.83^{b}$ \\
\hline SAr & 2.73 & 2.99 & 3.26 & 3.17 & 3.07 & 3.50 & 3.06 & 2.84 & 2.77 & 2.97 \\
\hline
\end{tabular}

Mean values in the same row with a different superscript differ significantly from each other at $p<.05$. 


\section{Guilt}

The interaction effect between SC and type of appeal was not statistically significant, $F(3$, $121)=.868, p=.460$ (see Table 5.28). This indicates that there is no significant difference in the effect of self-referencing or sources of evaluation on GAr between IndSC and IntSC.

There was also no statistically significant main effect for type of appeal, $F(3,121)=$ $1.480, p=.224$; but there was statistically significant main effect for $\mathrm{SC}, F(1,121)=$ $4.779, p=.031$ (see Table 5.27). This means that across type of appeal (self-referenced and other-referenced, internal and external) GAr scores do not differ, but these scores differ across IndSC and IntSC (see Section 5.6.4.2).

Table 5.28. Interaction of Self-Construal and Type of Appeal for Guilt

\begin{tabular}{llllll}
\hline & Type of appeal & N & Mean $^{*}$ & SE & \\
\hline \multirow{2}{*}{ IndSC } & Self-referenced guilt & 13 & 3.118 & .404 & \\
& Other-referenced guilt & 21 & 3.455 & .318 & \\
& Internal guilt & 11 & 3.821 & .446 & F $(3,121)=.868$, \\
& External guilt & 20 & 3.168 & .326 & $\mathrm{p}=.460$ \\
\cline { 2 - 4 } IntSC & Self-referenced guilt & 13 & 2.593 & .407 & \\
& Other-referenced guilt & 16 & 3.477 & .365 & \\
& Internal guilt & 23 & 2.694 & .304 & \\
& External guilt & 13 & 2.471 & .404 & \\
\hline
\end{tabular}

* Covariates appearing in the model are evaluated at the following values: GAp $=3.63$.

From the results of the analysis, hypotheses regarding guilt are confirmed as:

H6 (a) is not accepted: For independent self-construals, levels of emotional arousal elicited from a guilt appeal are not impacted by self-referencing. Specifically, they exhibit equal guilt arousal across the self-referenced and other-referenced appeal.

H6 (b) is accepted: For interdependent self-construals, levels of emotional arousal elicited from a guilt appeal are not impacted by self-referencing. Specifically, they exhibit equal guilt arousal across the self-referenced and other-referenced appeal. 
H7 (a) is not accepted: For independent self-construals, levels of emotional arousal elicited from a guilt appeal are not impacted by the evaluation source. Specifically, they exhibit equal guilt arousal across the internal and external appeal.

H7 (b) is accepted: For interdependent self-construals, levels of emotional arousal elicited from a guilt appeal are not impacted by the evaluation source. Specifically, they exhibit equal guilt arousal across the internal and external appeal.

\section{Shame}

The interaction effect between SC and type of appeal was not statistically significant, $F(3$, $127)=1.033, p=.380$ (see Table 5.29). This indicates that there is no significant difference in the effect of self-referencing or sources of evaluation on SAr between IndSC and IntSC.

There were also no statistically significant main effects for type of appeal, $F(3,127)=$ $.112, p=.953$ (see Table 5.27) or SC, $F(1,127)=.256, p=.613$ (see Section 5.6.4.2).

Table 5.29. Interaction of Self-Construal and Type of Appeal for Shame

\begin{tabular}{lllllll}
\hline & Type of appeal & N & Mean $^{*}$ & SE & \\
\hline \multirow{2}{*}{ IndSC } & Self-referenced shame & 14 & 2.819 & .330 & \\
& Other-referenced shame & 17 & 3.051 & .300 & \\
& Internal shame & 12 & 3.187 & .359 & \\
\multirow{3}{*}{ IntSC } & External shame & 14 & 3.238 & .331 & \multirow{2}{*}{$F(3,127)=1.033}$, \\
\cline { 2 - 4 } & Self-referenced shame & 21 & 3.347 & .269 & $\mathrm{p}=.380$ \\
& Other-referenced shame & 21 & 2.797 & .270 & \\
& Internal shame & 17 & 2.924 & .300 & \\
& External shame & 20 & 2.791 & .276 & \\
\hline
\end{tabular}

* Covariates appearing in the model are evaluated at the following values: $\mathrm{SAp}=4.48$.

From the results of the analysis, hypotheses regarding shame are confirmed as:

H8 (a) is not accepted: For independent self-construals, levels of emotional arousal elicited from a shame appeal are not reversely impacted by self-referencing. Specifically, they exhibit equal shame arousal across the self-referenced and other-referenced appeal. 
H8 (b) is accepted: For interdependent self-construals, levels of emotional arousal elicited from a shame appeal are not impacted by self-referencing. Specifically, they exhibit equal shame arousal across the self-referenced and other-referenced appeal.

H9 (a) is not accepted: For independent self-construals, levels of emotional arousal elicited from a shame appeal are not impacted by the evaluation source. Specifically, they exhibit equal shame arousal across the internal and external appeal.

H9 (b) is accepted: For interdependent self-construals, levels of emotional arousal elicited from a shame appeal are not impacted by the evaluation source. Specifically, they exhibit equal shame arousal across the internal and external appeal.

\section{Main effect of Self-referencing or Sources of evaluation}

ANCOVA tests were used to determine there is a difference between two self-referencing or sources of evaluation in GAr and SAr.

Table 5.30. Adjusted Means for Self-Referencing on Guilt/Shame Arousal

\begin{tabular}{lllllr}
\hline & Self-referencing & N & Mean & SE & \\
\hline \multirow{2}{*}{ Guilt } & Self-referenced guilt & 26 & $2.86^{\mathrm{a}}$ & .269 & $\mathrm{~F}(1,60)=3.030$, \\
& Other-referenced guilt & 37 & $3.47^{\mathrm{a}}$ & .269 & $\mathrm{p}=.087$ \\
\hline \multirow{2}{*}{ Shame } & Self-referenced shame & 35 & $3.17^{\mathrm{b}}$ & .210 & $\mathrm{~F}(1,70)=.601$, \\
& Other-referenced shame & 38 & $2.95^{\mathrm{b}}$ & .201 & $\mathrm{p}=.441$ \\
\hline
\end{tabular}

Covariates appearing in the model are evaluated at the following values: ${ }^{\mathrm{a}} \mathrm{GAp}=3.63 ;{ }^{\mathrm{b}} \mathrm{SAp}=4.45$.

Table 5.31. Adjusted Means for Source of Evaluation on Guilt/Shame Arousal

\begin{tabular}{lllllr}
\hline & Source of evaluation & N & Mean & SE & \\
\hline Guilt & Internal guilt & 34 & $3.04^{\mathrm{a}}$ & .271 & $\mathrm{~F}(1,64)=.104$, \\
& External guilt & 33 & $2.91^{\mathrm{a}}$ & .275 & $\mathrm{p}=.748$ \\
\hline \multirow{2}{*}{ Shame } & Internal shame & 29 & $2.99^{\mathrm{b}}$ & .232 & $\mathrm{~F}(1,60)=.025$, \\
& External shame & 34 & $2.94^{\mathrm{b}}$ & .214 & $\mathrm{p}=.874$ \\
\hline
\end{tabular}

Covariates appearing in the model are evaluated at the following values: ${ }^{\mathrm{a}} \mathrm{GAp}=3.63$; ${ }^{\mathrm{b}} \mathrm{SAp}=4.29$. 


\section{Guilt}

After adjusting for GAp, there was a marginally significant difference between selfreference and other-reference on GAr, $F(1,60)=3.030, p=.087: M=2.86$ and $M=3.47$, respectively (see Table 5.30); but no statistically significant difference between internal and external source of evaluation, $F(1,64)=.104, p=.748($ see Table 5.31).

\section{Shame}

After adjusting for SAp, there were no statistically significant differences between selfreference and other-reference, $F(1,70)=.601, p=.441($ see Table 5.30) and internal and external source of evaluation, $F(1,60)=.025, p=.874$, on SAr (see Table 5.31).

\subsubsection{Moderating effect of Personal cultural orientation}

A SEM multigroup analysis was performed to test $\mathrm{H} 10$ and $\mathrm{H} 11$. The main focus of the comparison across the two PCO groups was to establish whether any causal path in the hypothesised model (GAr/SAr $\rightarrow$ MC) differed significantly between IndPCO and ColPCO.

Table 5.32. Group Comparison on the Basis of Personal Cultural Orientation

\begin{tabular}{|c|c|c|c|c|}
\hline \multirow{2}{*}{ Paths } & \multicolumn{2}{|l|}{ IndPCO } & \multicolumn{2}{|l|}{ ColPCO } \\
\hline & Standard coefficients & $p$-value & Standard coefficients & $p$-value \\
\hline $\mathrm{GAr} \rightarrow \mathrm{MC}$ & .263 & $* * *$ & .354 & $* * *$ \\
\hline $\mathrm{SAr} \rightarrow \mathrm{MC}$ & .117 & ns & .361 & ** \\
\hline
\end{tabular}

ns: no significance, $* * p<.01, * * * p<.001$.

Table 5.33. Adjusted Means for Personal Cultural Orientation on Message

\section{Compliance}

\begin{tabular}{lllllr}
\hline & Personal cultural orientation & N & Mean & SE & \\
\hline Guilt & IndPCO & 71 & $3.41^{\mathrm{a}}$ & .184 & $\mathrm{~F}(1,127)=.600$, \\
& ColPCO & 59 & $3.63^{\mathrm{a}}$ & .203 & $\mathrm{p}=.440$ \\
\hline \multirow{2}{*}{ Shame } & IndPCO & 73 & $3.62^{\mathrm{b}}$ & .178 & $\mathrm{~F}(1,133)=.248$, \\
& ColPCO & 63 & $3.77^{\mathrm{b}}$ & .192 & $\mathrm{p}=.619$ \\
\hline
\end{tabular}

Covariates appearing in the model are evaluated at the following values: ${ }^{\mathrm{a}} \mathrm{GAr}=3.09 ;{ }^{\mathrm{b}} \mathrm{SAr}=3.01$. 


\section{Guilt}

The hypothesised model with multigroup analysis shows a good fit with the data encompassing IndPCO and ColPCO: $\chi^{2} / d f=1.56$; TLI $=.96 ; \mathrm{CFI}=.97 ; \mathrm{RMSEA}=.066$.

In the multigroup analysis, the path (GAr $\rightarrow \mathrm{MC})$ is significantly positive for each of these two PCO (IndPCO $\beta=.263, p=.000$; ColPCO $\beta=.354, p=.000)$. As shown in Table 5.32, the path is not significantly different across IndPCO and ColPCO (the $\chi^{2}$ difference test was nonsignificant, $\left.\Delta \chi_{(1)}^{2}=1.102, p=.294\right)$. As per the result, we cannot reject the constrained model. Thus, PCO does not moderate the relationship between GAr and $\mathrm{MC}$.

For comparison of means, ANCOVA tests were used to determine there is a difference between two PCO groups in MC. After adjusting for GAr scores, there was no significant difference between IndPCO and ColPCO on MC scores, $F(1,127)=.600, p=.440$, partial eta squared $\eta^{2}=.005$ (see Table 5.33).

Thus, H10 is accepted: The impact of guilt arousal on message compliance is not moderated by personal cultural orientation. Specifically, both (a) individualist and (b) collectivist individuals experiencing guilt are likely to comply with the message.

\section{Shame}

The hypothesised model with multigroup analysis shows a good fit with the data encompassing IndPCO and ColPCO: $\chi^{2} / d f=1.68 ; \mathrm{TLI}=.95 ; \mathrm{CFI}=.97 ; \mathrm{RMSEA}=.071$.

In this multigroup analysis, the path ( $\mathrm{SAr} \rightarrow \mathrm{MC}$ ) is significantly positive only for ColPCO $(\beta=.361, p=.004)$, but nonsignificant for IndPCO $(\beta=.117, p=.361)$. Hence, PCO does moderate the relationship between SAr and MC. Specifically, the relationship is not significant for individualists but significant for collectivists.

Again, ANCOVA tests were used to determine there is a difference between two PCO groups in MC. After adjusting for SAr scores, there was no significant difference between IndPCO and ColPCO on MC scores, $F(1,133)=.248, p=.619$, partial eta squared $\eta^{2}=$ .002 (see Table 5.33).

Thus, H11 is accepted: The impact of shame arousal on message compliance is moderated by personal cultural orientation. Specifically, (a) individualist individuals experiencing 
shame are not significantly likely to comply with the message, whereas (b) collectivist individuals experiencing shame are likely to do so.

\subsubsection{Moderation effect of Type of emotion}

In order to test Hypothesis 12, a SEM multigroup analysis was performed to establish whether GAr or SAr predict MC differently. The hypothesised model with multigroup analysis shows a good fit with the data encompassing GAr and SAr: $\chi^{2} / d f=1.98$; TLI $=$ $.96 ; \mathrm{CFI}=.98 ; \mathrm{RMSEA}=.061$.

The results of SEM path analysis show that the total effect of Emotional arousal (EAr) towards MC for GAr is stronger than for SAr: $\beta_{\mathrm{G}}=.45>\beta_{\mathrm{S}}=.36$ (both $p \mathrm{~s}<.01$ ). However, the difference is not significant across type of emotion (the $\chi^{2}$ difference test was nonsignificant, $\left.\Delta \chi^{2}(3)=1.426, p=.699\right)$.

As per the result, $\mathrm{H} 12$ is not accepted: Type of emotion does not moderate the relationship between emotional arousal (guilt versus shame, respectively) and message compliance.

\subsubsection{Interaction effect of Personal cultural orientation and Type of emotion}

A two-way ANCOVA was conducted to explore the impact of PCO (IndPCO and ColPCO) and type of emotion (guilt and shame) on MC after adjusting for guilt/shame EAr scores. Results of a two-way ANCOVA and main effect tests for MC can be found in Table 5.34.

Table 5.34. Interaction of Personal Cultural Orientation and Type of Emotion

\begin{tabular}{|c|c|c|c|c|c|}
\hline & Interaction & $\mathbf{N}$ & Mean* & SE & \\
\hline \multirow{2}{*}{ Guilt } & IndPCO & 71 & 3.41 & .196 & \multirow{4}{*}{$\begin{array}{r}F(1,261)=.001 \\
p=.982\end{array}$} \\
\hline & ColPCO & 59 & 3.59 & .216 & \\
\hline \multirow{2}{*}{ Shame } & IndPCO & 73 & 3.63 & .191 & \\
\hline & ColPCO & 63 & 3.80 & .206 & \\
\hline \multirow{4}{*}{ Main effect } & IndPCO & 144 & 3.52 & .138 & $F(1,261)=.722$ \\
\hline & ColPCO & 122 & 3.70 & .150 & $p=.396$ \\
\hline & Guilt & 130 & 3.50 & .144 & $F(1,261)=1.064$ \\
\hline & Shame & 136 & 3.71 & .140 & $p=.303$ \\
\hline
\end{tabular}

\footnotetext{
* Covariates appearing in the model are evaluated at the following values: EAr $=3.05$.
} 
The interaction effect between PCO and type of emotion was not statistically significant, $F(1,261)=.001, p=.982$. This indicates that there is no significant difference in the effect of PCO on MC between guilt and shame.

There was no statistically significant main effect for $\mathrm{PCO}, F(1,261)=.722, p=.396$ and for type of emotion, $F(1,261)=1.064, p=.303$. This means that collectivist individuals and individualist individuals do not differ in terms of message compliance. Also, there is no significant difference in message compliance scores for guilt and shame type of emotion (see Section 5.6.4.5).

\subsubsection{Additional Research Findings}

\subsubsection{Moderating effect of Emotional arousal intensity}

A Pearson Chi-square test for independence indicated a significantly medium association between the level of guilt/shame EAr intensity and the type of coping response, $\chi^{2}(4, n=$ 266) $=30.199, p=.000$, Cramer's $V=.238$. Specifically, low emotional arousals lead mostly to maladaptive coping (53.3\%), medium and high emotional arousals lead mostly to adaptive coping (51.2\% and $75.5 \%$ respectively, see Table 5.35).

Table 5.35. Level of Emotional Arousal and Type of Coping Response

\begin{tabular}{llrrrr}
\hline \multirow{2}{*}{ Emotional arousal intensity } & \multicolumn{3}{c}{ Coping response } & \multirow{2}{*}{ Total } \\
\cline { 2 - 5 } & Count & 72 & 20 & 43 & 135 \\
\multirow{3}{*}{ Low } & Expected Count & 55.8 & 17.3 & 61.9 & 135.0 \\
& \% within Low-Medium-High & 53.3 & 14.8 & 31.9 & 100.0 \\
\cline { 2 - 5 } & Count & 31 & 9 & 42 & 82 \\
\multirow{4}{*}{ Medium } & Expected Count & 33.9 & 10.5 & 37.6 & 82.0 \\
& \% within Low-Medium-High & 37.8 & 11.0 & $\mathbf{5 1 . 2}$ & 100.0 \\
\cline { 2 - 5 } & Count & 7 & 5 & 37 & 49 \\
& Expected Count & 20.3 & 6.3 & 22.5 & 49.0 \\
& \% within Low-Medium-High & 14.3 & 10.2 & $\mathbf{7 5 . 5}$ & 100.0 \\
\hline
\end{tabular}

Thus, levels of emotional intensity of guilt or shame arousal (low/medium/high) have an impact on coping responses (maladaptive/adaptive). Specifically, the higher the intensity of emotional arousal (medium and high), the greater the adaptive coping response. The 
finding is consistent with previous studies in threat appeals: emotional responses (stronger/weaker) impact on coping responses (adaptive/maladaptive). That is, stronger emotional responses lead an individual to use an adaptive response (Folkman et al., 1979; Tanner et al., 1991).

\subsubsection{Moderating effect of Coping response type}

One-way ANOVA tests were used to determine the relationship between type of coping response and MC as shown in Table 5.36. The differences between the type of coping response and the creation of message compliance were significant, $F(2,263)=21.333, p$ $=.000$. Post-hoc comparisons using the Hochberg test indicated that the mean score for the maladaptive coping responses $(M=2.85, S D=1.537)$ was significantly different from either of the other coping responses: the adaptive coping $(M=4.23, S D=1.608, p=$ $.000)$ and the neutral coping $(M=3.76, S D=1.855, p=.013)$. The neutral coping responses did not differ significantly from the adaptive coping responses $(p=.352)$.

Table 5.36. Mean Message Compliance by Type of Coping Response

\begin{tabular}{llcccr}
\hline & Coping response & N & Mean & SD & \\
\hline \multirow{4}{*}{ MC } & Maladaptive & 110 & 2.85 & 1.537 & \\
& Neutral & 34 & 3.76 & 1.855 & $\mathrm{~F}(2,263)=21.333$, \\
& Adaptive & 122 & 4.23 & 1.608 & $\mathrm{p}=.000$ \\
& Total & $\mathbf{2 6 6}$ & $\mathbf{3 . 6 0}$ & $\mathbf{1 . 7 3 2}$ & \\
\hline
\end{tabular}

Therefore, the type of coping response (maladaptive/adaptive) has an impact on message compliance (lesser/greater). Specifically, neutral and adaptive coping responses lead to greater message compliance than maladaptive coping responses.

\subsubsection{Further dependent measures}

Bivariate correlations were used to test the relationships between the dependent variables: message compliance (MC), binge drinking intention (BDI), attitude towards message (MAt), and attitude towards ad (AAt). Table 5.37 reports zero-order Pearson correlations for the dependent measures.

There was a medium, negative correlation between MC and BDI $(r=-.351, p<.001)$, with high levels of compliance with the message associated with lower levels of intention 
to binge drinking; large, positive correlations between MC and MAt $(r=.609, p<.001)$, and MC and AAt $(r=.592, p<.001)$, with high levels of compliance with the message associated with higher levels of attitude towards the message as well as the ad. An inspection of the zero-order correlation $(r=.806, p<.001)$ suggested that there was a very strong, positive correlation between MAt and AAt.

Table 5.37. Zero-order Pearson Correlations for Dependent Measures

\begin{tabular}{lcccc}
\hline & MC & BDI & MAt & AAt \\
\hline Message compliance & - & & & \\
Binge drinking intention & $-.351^{* * *}$ & - & & \\
Attitude towards message & $.609^{* * *}$ & $-.258^{* * *}$ & - & \\
Attitude towards ad & $.592^{* * *}$ & $-.263^{* * *}$ & $.806^{* * *}$ & - \\
\hline
\end{tabular}

$n=266 ; * * * p<.001$.

For actual behaviour, with regards to interest in learning more about binge drinking by clicking on the hyperlink to go to a website offering additional information about responsible drinking, there was a significant difference in MC scores for students who did and did not click, $t(264)=2.803, p=.005$, eta squared $=.029$. Specifically, those who did $(M=4.01, S D=1.633)$ scored higher than those who did not $(M=3.39, S D=1.749)$. Remarkably, $72.5 \%(n=66)$ of those who clicked the link reported having viewed the website for more information, displaying the strong intention-behaviour link among potential individuals who showed compliance with the emotional appeal message.

The results reveal the predicted pattern regarding the correlations between the group of dependent variables. Three separate dependent variables: compliance, intention, and attitude, together provide convergent evidence in support of the proposed theorising that individuals experiencing guilt or shame are likely to comply with the health emotional appeal message. That is, the higher the emotional arousal, the greater the health message compliance, the lower the intentions to binge drink, the greater the attitudes towards the ad and the heath message featured in the ad.

\subsubsection{Demographics}

Previous research suggested that individuals' arousing and coping with the emotions might vary by demographic factors (Else-Quest, Higgins, Allison, \& Morton, 2012; Kim 
\& Johnson, 2013). Therefore, in the current study of health communications using guilt and shame appeal, the model additionally attempts to examine the moderating effect of demographic factors on the paths of EAp (GAp/SAp) $\rightarrow$ EAr $(\mathrm{GAr} / \mathrm{SAr}) \rightarrow \mathrm{CR} \rightarrow \mathrm{MC}$ that have been used in one or more of the previous studies. Demographics such as gender and alcohol use disorder are often examined in anti-drinking messages (e.g., Treeby \& Bruno, 2012). As a result, they have also been incorporated into the analysis.

\section{Gender}

The results of the moderating effect of gender are summarised in Table 5.38. Gender moderates the relationship between GAp and GAr, GAr and CR, and GAr and MC for guilt, and the relationship between SAp and SAr, SAr and MC for shame. However, no moderating effect of gender was found on the relationship between CR and MC for guilt; the relationship between SAr and CR, CR and MC for shame.

The strength of the relationship between GAp/SAp and GAr/SAr, GAr and CR, and $\mathrm{GAr} / \mathrm{SAr}$ and $\mathrm{MC}$ is significantly greater for males than for females (the $\chi^{2}$ difference test was significant, $p \mathrm{~s}<.05)$.

Table 5.38. Group Comparison on the Basis of Gender

\begin{tabular}{|c|c|c|c|c|}
\hline \multirow{2}{*}{ Paths } & \multicolumn{2}{|l|}{ Male } & \multicolumn{2}{|l|}{ Female } \\
\hline & Standard coefficients & $p$-value & Standard coefficients & $p$-value \\
\hline $\mathrm{GAp} \rightarrow \mathrm{GAr}$ & $.717^{\mathrm{a}}$ & $* * *$ & $.383^{b}$ & $* * *$ \\
\hline $\mathrm{GAr} \rightarrow \mathrm{CR}$ & $.601^{\mathrm{a}}$ & *** & $.049^{b}$ & ns \\
\hline $\mathrm{GAr} \rightarrow \mathrm{MC}$ & $.746^{\mathrm{a}}$ & $* * *$ & $.144^{b}$ & ns \\
\hline $\mathrm{CR} \rightarrow \mathrm{MC}$ & .172 & * & .195 & * \\
\hline SAp $\rightarrow$ SAr & $.765^{\mathrm{a}}$ & $* * *$ & $.552^{\mathrm{b}}$ & $* * *$ \\
\hline $\mathrm{SAr} \rightarrow \mathrm{CR}$ & .469 & *** & .315 & ** \\
\hline $\mathrm{SAr} \rightarrow \mathrm{MC}$ & $.428^{a}$ & $* * *$ & $.048^{b}$ & ns \\
\hline $\mathrm{CR} \rightarrow \mathrm{MC}$ & .221 & ** & .298 & ** \\
\hline
\end{tabular}

ns: no significance, $* p<.05, * * p<.01, * * * p<.001 ; \beta$ values in the same row with a different superscript differ significantly from each other at $p<.05$.

Furthermore, ANCOVA tests were used to determine there is a difference between males and females in MC. For guilt, after adjusting for GAr scores, there was no significant difference between genders on MC scores, $F(1,127)=1.956, p=.164$. Similarly, for 
shame, after adjusting for SAr scores, there was also no significant difference between males and females on MC scores, $F(1,133)=3.010, p=.085$ (see Table 5.39). The results suggest that there was no difference between males and females experiencing guilt or shame with regards to compliance with the anti-drinking message.

Table 5.39. Adjusted Means for Gender on Message Compliance

\begin{tabular}{lllllr}
\hline & Gender & N & Mean & SE & \\
\hline Guilt & Male & 54 & $3.73^{\mathrm{a}}$ & .206 & $\mathrm{~F}(1,127)=1.956$, \\
& Female & 76 & $3.35^{\mathrm{a}}$ & .173 & $\mathrm{p}=.164$ \\
\hline \multirow{2}{*}{ Shame } & Male & 55 & $4.04^{\mathrm{b}}$ & .233 & $\mathrm{~F}(1,133)=3.010$, \\
& Female & 81 & $3.48^{\mathrm{b}}$ & .192 & $\mathrm{p}=.085$ \\
\hline
\end{tabular}

Covariates appearing in the model are evaluated at the following values: ${ }^{\mathrm{a}} \mathrm{GAr}=3.09 ;{ }^{\mathrm{b}} \mathrm{SAr}=3.01$.

\section{Alcohol use disorder}

Table 5.40 reports the results of multigroup SEM for the moderating effect of alcohol use disorder (as measured by AUDIT). The level of alcohol use disorder only moderates the path $\mathrm{SAr} \rightarrow \mathrm{MC}$ for shame. That is, the relationship between SAr and MC is significant for individuals with a high-risk level of alcohol use disorder but is not significant for those with a low-risk level.

Table 5.40. Group Comparison on the Basis of Alcohol Use Disorder

\begin{tabular}{|c|c|c|c|c|}
\hline \multirow{2}{*}{ Paths } & \multicolumn{2}{|c|}{ Non- or low-risk } & \multicolumn{2}{|c|}{ At-risk or high-risk } \\
\hline & Standard coefficients & $p$-value & Standard coefficients & $p$-value \\
\hline $\mathrm{GAp} \rightarrow \mathrm{GAr}$ & .496 & $* * *$ & .596 & $* * *$ \\
\hline $\mathrm{GAr} \rightarrow \mathrm{CR}$ & .293 & $* * *$ & .322 & $* \star \star$ \\
\hline $\mathrm{GAr} \rightarrow \mathrm{MC}$ & .323 & $* * *$ & .369 & $* * *$ \\
\hline $\mathrm{CR} \rightarrow \mathrm{MC}$ & .250 & $* * *$ & .278 & $* * *$ \\
\hline SAp $\rightarrow$ SAr & .587 & $* * *$ & .638 & $* * *$ \\
\hline $\mathrm{SAr} \rightarrow \mathrm{CR}$ & .371 & $* * *$ & .353 & $* \star \star$ \\
\hline $\mathrm{SAr} \rightarrow \mathrm{MC}$ & .063 & ns & .505 & $* * *$ \\
\hline $\mathrm{CR} \rightarrow \mathrm{MC}$ & .169 & ** & .262 & ** \\
\hline
\end{tabular}

ns: no significance, $* * p<.01, * * * p<.001$.

Again, ANCOVA tests were used to determine there is a difference between two levels of alcohol use disorder in MC. After adjusting for GAr/SAr scores, there was significant 
difference between the two levels on MC scores: guilt $F(1,127)=5.989, p=.016$, partial eta squared $\eta^{2}=.045$; shame $F(1,133)=8.845, p=.003$, partial eta squared $\eta^{2}=.062$ (see Table 5.41). The results suggest that individuals with low- and high-risk levels of alcohol use disorder respond differently to message compliance. Individuals with a lowrisk level of alcohol use disorder showed greater compliance with the anti-drinking message than those with a high-risk level. This finding is consistent with previous studies that high-risk drinkers are likely to be more resistant to messages suggesting change (e.g., Logan, Kilmer, \& Marlatt, 2010).

Table 5.41. Adjusted Means for AUDIT Level on Message Compliance

\begin{tabular}{lllllr}
\hline & Risk level & N & Mean & SD & \\
\hline Guilt & Non- or low-risk & 56 & $3.88^{\mathrm{a}}$ & .198 & $\mathrm{~F}(1,127)=5.989$, \\
& At-risk or high-risk & 74 & $3.24^{\mathrm{a}}$ & .172 & $\mathrm{p}=.016$ \\
\hline \multirow{2}{*}{ Shame } & Non- or low-risk & 63 & $4.15^{\mathrm{b}}$ & .212 & $\mathrm{~F}(1,133)=8.845$, \\
& At-risk or high-risk & 73 & $3.29^{\mathrm{b}}$ & .197 & $\mathrm{p}=.003$ \\
\hline
\end{tabular}

Covariates appearing in the model are evaluated at the following values: ${ }^{\mathrm{a}} \mathrm{GAr}=3.09 ;{ }^{\mathrm{b}} \mathrm{SAr}=3.01$.

\subsection{Summary of Hypotheses Testing}

This chapter outlined the sample characteristics of the survey, evaluated the measurement model based on CFA, and tested the hypotheses using SEM and ANCOVA. Most of the hypotheses are accepted as significant or partially significant, while only one is not accepted. Specifically, five out of 12 hypotheses are fully supported, six partially supported, and one rejected in this study. Table 5.42 presents a summary of the results of the hypotheses testing.

Table 5.42. Summary of Hypotheses Testing

\section{Hypotheses}

\section{Outcome}

H1: (a) Guilt or (b) shame appeals have an impact on respective guilt or Accepted shame arousals. Specifically, with high levels of emotional appeals associated with high levels of emotional arousals.

H2: (a) Guilt or (b) shame arousals have an impact on message Accepted compliance. Specifically, with high levels of emotional arousals associated with high levels of the message compliance. 
H3: Coping responses will mediate the relationship between guilt or shame arousal and message compliance.
Accepted: Partial mediation

of coping responses.

H4 (a): Levels of emotional arousal elicited from guilt appeal are Rejected impacted by regulatory focus. Specifically, prevention-focused individuals will exhibit higher guilt arousal than their promotion-focused counterparts.

H4 (b): Levels of emotional arousal elicited from a shame appeal are Accepted impacted by regulatory focus. Specifically, prevention-focused individuals will exhibit higher shame arousal than their promotion-focused counterparts.

H5 (a): For independent self-construals, they will exhibit higher guilt Accepted arousal than their interdependent counterparts (regardless of appeal type).

H5 (b): For interdependent self-construals, they will exhibit higher shame Rejected arousal than their independent counterparts (regardless of appeal type).

H6 (a): For independent self-construals, levels of emotional arousal Rejected elicited from a guilt appeal are impacted by self-referencing. Specifically, they will exhibit higher guilt arousal for a self-referenced appeal than for an other-referenced appeal.

H6 (b): For interdependent self-construals, levels of emotional arousal Accepted elicited from a guilt appeal are not impacted by self-referencing. Specifically, they will exhibit equal guilt arousal for a self-referenced appeal as for an other-referenced appeal.

H7 (a): For independent self-construals, levels of emotional arousal Rejected elicited from a guilt appeal are impacted by the evaluation source. Specifically, they will exhibit higher guilt arousal for an internal appeal than for an external appeal.

H7 (b): For interdependent self-construals, levels of emotional arousal Accepted elicited from a guilt appeal are not impacted by the evaluation source. Specifically, they will exhibit equal guilt arousal for an internal appeal as for an external appeal.

H8 (a): For independent self-construals, levels of emotional arousal Rejected elicited from a shame appeal are reversely impacted by self-referencing. Specifically, they will exhibit higher shame arousal for an otherreferenced appeal than for a self-referenced appeal.

H8 (b): For interdependent self-construals, levels of emotional arousal Accepted elicited from a shame appeal are not impacted by self-referencing. Specifically, they will exhibit equal shame arousal for a self-referenced appeal as for an other-referenced appeal. 
H9 (a): For independent self-construals, levels of emotional arousal elicited from a shame appeal are impacted by the evaluation source.

Specifically, they will exhibit higher shame arousal for an internal appeal than for an external appeal.

H9 (b): For interdependent self-construals, levels of emotional arousal elicited from a shame appeal are not impacted by the evaluation source. Specifically, they will exhibit equal shame arousal for an internal appeal as for an external appeal.

H10: The impact of guilt arousal on message compliance is not Accepted moderated by personal cultural orientation. Specifically, individuals experiencing guilt are likely to comply with the message (regardless of either (a) individualist or (b) collectivist orientations).

H11: The impact of shame arousal on message compliance is moderated Accepted by personal cultural orientation. Specifically, (a) individualist individuals experiencing shame are not likely to comply with the message, whereas (b) collectivist individuals experiencing shame are likely to do so.

H12: Guilt arousal is more likely than shame arousal to lead to message Rejected compliance.

\begin{tabular}{|c|c|}
\hline Additional findings & Outcome \\
\hline $\begin{array}{l}\text { Q1: Does personal cultural orientation have an impact on the respective } \\
\text { relationships of guilt/shame arousal and message compliance? }\end{array}$ & $\begin{array}{l}\text { No interaction effect of } \\
\text { personal cultural orientation } \\
\text { and emotion type. }\end{array}$ \\
\hline $\begin{array}{l}\text { Q2: Does level of emotional intensity of guilt or shame arousal } \\
\text { (low/medium/high) have an impact on coping responses } \\
\text { (maladaptive/adaptive)? }\end{array}$ & $\begin{array}{l}\text { Yes: High- and medium- } \\
\text { level emotional arousals } \\
\text { produce the most adaptive } \\
\text { coping response followed by } \\
\text { low emotional arousals. }\end{array}$ \\
\hline $\begin{array}{l}\text { Q3: Does type of coping response (maladaptive/adaptive) have an } \\
\text { impact on message compliance (lesser/greater)? }\end{array}$ & $\begin{array}{l}\text { Yes: Adaptive coping } \\
\text { responses produce greater } \\
\text { message compliance than } \\
\text { maladaptive coping } \\
\text { responses. }\end{array}$ \\
\hline
\end{tabular}

Q4: Do attitude, behavioural intention, and compliance with the message Medium-strong correlations. highly correlate?

Q5: Are there differences in message compliance between males and females?

No moderation effect of gender.

Q6: Are there differences in message compliance between individuals with low- versus high-risk levels of alcohol use disorder?

Rejected

Accepted 
The analyses discussed in this chapter have aided in the achievement of the present study's objectives, namely: (i) the identification of the coping response as the mediator deemed important for self-conscious emotional guilt and shame appeal message compliance, and (ii) the investigation of self-construal, regulatory focus and personal cultural orientation of individuals as the moderators towards emotional arousals and the message compliance. The next chapter delves into these results in more detail and discusses their theoretical implications. 


\section{Chapter 6. Discussion of Results}

\subsection{Introduction}

In this chapter, the study's hypotheses are discussed in-depth based on the results presented in Chapter 5. Some additional noteworthy relationships outside those hypothesised are also considered. These results are discussed in light of previous studies, particularly within the emotions, health communications and moderate drinking literature.

The results indicate that the guilt and shame appeals identified affected individuals' compliance with the health message in different ways. The findings can be summarised as follows:

1. Guilt and shame appeals positively influence the respective guilt and shame arousals;

2. Guilt and shame arousals had direct and positive effects on message compliance;

3. Guilt and shame arousals indirectly influence message compliance through coping responses. In other words, coping responses mediate the relationship between guilt or shame arousals and message compliance;

4. Levels of guilt and shame arousals are moderated by regulatory focus and selfconstrual;

5. There were no interactive effects of self-construal with self-referencing or sources of evaluation on guilt and shame arousals;

6. The impact of shame arousals on message compliance was moderated, but not for guilt arousals by personal cultural orientation;

7. There was no significant difference of guilt versus shame arousals in message compliance.

As well as findings for the relationships between each of the guilt and shame appeals and message compliance as hypothesised, there are also several additional findings regarding the moderating effects of interaction of emotional type and personal cultural orientation, and demographics on message compliance. In order to discuss these findings, this chapter is structured based on the results laid out above, beginning with a discussion on guilt and shame appeals as antecedents of guilt and shame arousals, and followed by the relationships between these guilt/shame arousals and message compliance. Then, the moderating role of personal cultural orientation in the relationships between the 
guilt/shame arousals and message compliance, along with the indirect relationships derived from these relationships (through coping responses) are discussed. Lastly, the moderating roles of regulatory focus and self-construal in guilt and shame arousing are also discussed.

\subsection{Effects of Guilt and Shame on Behavioural Intention}

\subsubsection{Emotional Appeals and Emotional Arousals}

The results of this study indicate that guilt and shame emotional appeals significantly and positively impact respective guilt and shame emotional arousals from the binge drinking health message. This means that high levels of guilt and shame emotional appeals are associated with high levels of guilt and shame emotional arousals. The finding supports the view that emotional appeals have a strong impact on the level of emotion aroused (e.g., guilt, shame) in individuals. In the current study, guilt and shame appeals were based on the notion that guilt focuses on the behaviour and shame on the self, respectively (Lewis, 1971; Tangney \& Dearing, 2002). Therefore, guilt and shame in this study were aroused from generalised guilt- and shame-eliciting tactics (e.g., based on the nature of guilt, i.e., specific behaviour and shame, i.e., global self) rather than contextual tactics (e.g., reference to specific elements, i.e., alcohol use disorder) (Kim et al., 2011). It is worth noting in the study that the manipulation check of the health message ensured guilt appeal aroused significantly greater felt emotion of guilt than shame and shame appeal aroused significantly greater felt emotion of shame than guilt. By doing that, the current study had further clarified the distinction between emotional appeals and emotional arousals, a clarification that many theoretical models pertaining to emotions lack (LaTour \& Rotfeld, 1997). The issue constitutes a dissimilar body of work (e.g., emotional appeal versus emotional arousal) and therefore relating one study to another is often difficult.

Overall, the study's result is consistent with Carey, McDermott, and Sarma's (2013) findings of meta-analysis which suggest that the link between threat appeals and fear arousals elicited is definitely strong. Theoretically, self-conscious emotional appeals aim to modify behavioural intentions by increasing negative outcomes as a threat posed to personal notions of self-integrity. Thus, the present study provides evidence for the premise that guilt and shame emotional appeal message exposures were resulting in elevated guilt and shame emotional responses, respectively. 


\subsubsection{Emotional Arousals and Message Compliance}

The literature on emotion and behavioural intention has provided mixed evidence of the impact of emotion on individuals' behavioural intentions towards health messages, specifically, an inverted U-shaped relationship between guilt and intentions exists. Some studies have found evidence that supports the inverse-U relationship (Coulter et al., 1999; Coulter \& Pinto, 1995; Ghingold \& Bozinoff, 1982; O'Keefe, 2002; Pinto \& Priest, 1991; Pinto \& Worobetz, 1992); however, others did not support the inverse-U effect but rather supported a linear effect (Cotte et al., 2005; Lindsey, 2005; Turner \& Underhill, 2012). The findings of the present study align with the latter and reveal that emotion plays a major role in determining behavioural change towards the health message. That is, guilt and shame appeals positively influence respective guilt and shame arousals; in turn, guilt and shame arousals positively influence message compliance. In other words, the higher the emotional appeals, the higher the emotional arousals and, subsequently, the greater the compliance with the health message.

The possibility of an inverted U-shaped relationship between the levels of emotional arousals and message compliance was investigated. The findings of the examination of this curvilinear relationship revealed no inverse- $U$ effect observed in the data ${ }^{11}$, and thus provided no evidence in supporting Coulter and Pinto's (1995) conclusion that an inverted U-shaped curvilinear relationship exists in this context. This inconsistency could be explained with consideration of coping response. As findings of open-ended question coping response suggest, individuals who feel guilt or shame about their integrity of self may not be engaged in a maladaptive coping response even when the emotion is strong (see more in Section 6.2.3 below). Message receivers conform to social standards and withhold defensive emotional responses in their pathological tendencies in order to preserve their identity and maintain acceptance in their social bonds (Silfver-Kuhalampi, 2009; Silfver, 2007). For example, a participant expresses their thoughts, and feelings experienced in response to the binge drinking stimulus ad:

\footnotetext{
${ }^{11}$ Linearity was tested by use of ANOVA test of linearity which computes both the linear and nonlinear components of a pair of variables whereby nonlinearity is significant if the $F$ significance value for the nonlinear component is below .05 (Garson, 2012). All the value significances were above .05 ( $p=.331$ for guilt, $p=.599$ for shame) confirming linear relationships (constant slope) between emotional arousals and message compliance.
} 
"Made the viewer feel like shit this could be me in that photo and has struck a c[h]ord with getting drunk to me." (Respondent 7, European New Zealander, male, 25 years old)

The linear relationship between guilt arousal (and shame arousal as well) and message compliance differs from previous studies on guilt appeals in pro-social and charitable donation behaviour. The extant studies found the 'boomerang effect', that is, moderately intense guilt appeals are most effective, whereas highly intense guilt appeals lead consumers to respond negatively towards the advocated message, as they evoke anger, resentment and annoyance (Coulter et al., 1999; Coulter \& Pinto, 1995; Moore \& Harris, 1996). The effect is consistent with the inverted-U relationship between an ad's intensity and its effectiveness derived from research in fear appeals (Bennett, 1996). This discrepancy, however, can be attributed to the past studies evaluating emotional appeals instead of single emotional arousals (LaTour \& Rotfeld, 1997). This study, on the other hand, examined, separately, distinct emotional guilt versus shame arousals resulting from emotional appeals. As previously mentioned, messages designed to elicit highly intense levels of guilt may actually evoke shame feelings unintentionally or a combination of shame and guilt (Bennett, 1998; Boudewyns et al., 2013). By isolating the effect of an unintended emotion aroused from the appeal, the present study was able to discover that guilt and shame arousal via health communications is likely to be productive.

This current study also found that coping responses are the mechanisms through which guilt and shame responses persuade the adoption of health messages. That is, guilt and shame arousals influence coping responses and, ultimately, message compliance. The effectiveness of guilt and shame arousals relates strongly to their stimulation of adaptive coping response where health messages are accepted by the individual. Existence of the effect of mediation is one of the reasons explaining mixed findings in the past research. The study's finding suggests that individuals who feel guilt or shame aroused from health communications are likely to comply with the health message as a result of a threat posed to their personal notions of self-integrity (Leary \& Baumeister, 2000; Sznycer et al., 2016). Interestingly, the finding not only supports the notion that guilt tends to create more positive behaviours (Graton, Ric, \& Gonzalez, 2016; Saintives \& Lunardo, 2016; Tangney et al., 2007), but also critiques the rationale that shame is inherently maladaptive (Abe, 2004; Lindsay-Hartz, 1984; Stuewig et al., 2010). Rather, shame essentially 
motivates socially positive behaviours and efforts towards self-improvement (de Hooge et al., 2010; Gausel \& Leach, 2011; Sznycer et al., 2016). As Sznycer et al. (2016) explained: shame, although unpleasant, serves the adaptive function of defending against the social devaluation by preventing actions that would lead to more devaluation than benefits.

Another explanation to the inconsistency between this study's findings and those of previous studies is that the present study looks specifically into the health communications, which differs from the pro-social or charitable donations. Such that, at the individual level, the health communications involves stimulating message viewers for the benefit of themselves while the charitable donations involves motivating message viewers for the benefit of others. This explanation is supported by the finding of the marginal difference of self-reference versus other-reference in guilt arousal discussed in Section 5.6.4.3. Therefore, it can be seen that in the health communications context, guilt appeals operate differently due, mainly, to the interaction between the self-evaluative type of such an emotion and the personal significance in the context.

\subsubsection{Emotional Arousals and Coping Responses}

The positive relationship between guilt and shame emotional arousals and their coping responses towards the emotions shows that the higher intensity of guilt or shame individuals feel, the more adaptively they responded to the emotional appeal message. This result confirms Tanner et al.'s (1991) study in the fear appeal, where the authors found positive correlation between emotional response and coping response. In addition, the present study extends the mentioned study on fear by finding a positive directional relationship within the context of self-conscious emotions.

There are mixed findings regarding emotions, with Dickinson and Holmes (2008); Schoenbachler and Whittler (1996) found no relationship between emotional response and coping response, while Ang and Low (2000) and Tanner et al. (1991) found the relationship exists. In a study investigating social and physical types of threat on message effectiveness, Dickinson and Holmes (2008) found that low and moderate threats were the most effective at creating an adaptive coping response, and this was true of both social and physical threat communications. It is noted that in the mentioned study, emotional responses (arousals) were based on the summed score across disgust, guilt, stress and anger. The present study examining guilt separately from shame, however, supports the 
linear theory advocated by Folkman et al. (1979) and Tanner et al. (1991) which suggests that in terms of persuasiveness, stronger emotional responses lead to individuals using an adaptive response and, ultimately, message acceptance (Ang \& Low, 2000). From the present study's findings, it is clear that intense emotional arousals in general were significantly more likely to stimulate an adaptive coping response. The result confirms that individuals' coping responses are positively influenced by their guilt or shame arousals. That is, with a higher arousal of guilt or shame, individuals respond more adaptively than with a lower level of emotional arousal to health messages. This present finding is supported by previous studies, which found that the level of emotions is a significant factor influencing consumers' coping responses to these emotions (Ang \& Low, 2000; Folkman et al., 1979; Lwin \& Phau, 2011, 2014; Tanner et al., 1991).

In this study, the coping response was measured by an open-ended question where the participants freely described the thoughts and feelings that they experienced in response to the binge drinking stimulus ad. Coping responses were then categorised into three types: adaptive, neutral, and maladaptive coping (Dillard \& Peck, 2000). Accordingly, the study further found support for the premise that emotional intensity can influence the type of coping response to emotions and, subsequently, influence message compliance. This suggests that the coping response type moderates the effect of emotional arousal intensity as a categorical variable instead of a continuous variable. That is, the higher the guilt or shame arousal intensity individuals feel, the more likely they would respond adaptively to the health message, and the greater their compliance with the message. Participants reported the adaptive coping response when they felt highly intense guilt or shame arousing from guilt or shame appeals, followed by when they felt moderately intense emotional arousals. The maladaptive coping response resulted with the low intense emotional arousals.

\subsubsection{Coping Responses and Message Compliance}

The results of this study also indicate that the coping response significantly and positively impacts on compliance with the health message. This finding supports the view that individuals with an adaptive coping response are more likely to comply with the advocated message. The finding underscores the importance of the coping response as a key factor in motivating people to follow a recommended action advocated in the health message. This result is similar to those previous studies in the fear appeal context, which 
concluded that there is a significant relationship between the coping response and attitude towards the behaviour of smoking (Dickinson \& Holmes, 2011) or contracting maladaptive behaviours of sexually transmitted diseases (STDs, Tanner et al., 1991). That is, the higher coping response (stronger adaptive coping) results in a negative attitude towards the behaviour of smoking or contracting maladaptive behaviours of STDs. Thus, stimulating adaptive coping responses that create a positive attitude towards promoted messages is important for impacting on intended behaviour.

In effect, health communicators must not rely only on guilt or shame arousals as a strategy to secure individuals' message compliance but also on coping responses. When an emotional guilt or shame arousal induces individuals to recognise a threat posed to their personal notions of self-integrity (Leary \& Baumeister, 2000), they search for ways to deal with the threat; this is referred to as an adaptive coping response (Schoenbachler $\&$ Whittler, 1996). An adaptive coping response means that the health promotion message is being adopted as a result of the rational cognitive processes. Adaptively, the message receivers have an intention to improve their health and acknowledge the health message. Thus, this research indicates that by triggering adaptive coping responses when using guilt and shame appeals, health communications are likely to result in a favourable attitude towards the persuasive message and, ultimately, compliance to the message.

Moreover, coping responses in this research, besides being a continuous variable, were divided into three different types as categories (Dillard \& Peck, 2000). Accordingly, depending on the type of coping responses (adaptive, neutral, or maladaptive coping), message compliance may vary. Specifically, neutral and adaptive coping responses lead individuals to greater message compliance than maladaptive coping responses. In other words, when individuals do not cope maladaptively to the health emotional appeal message, they are more likely to comply with the message and behave positively.

\subsubsection{Mediating Effect of Coping Responses}

In mediation, there are two effects. Full mediation occurs when there is a significant indirect effect of the independent variable on a dependent variable via the mediator (sometimes referred to as a mediated effect), but there is no significant direct effect of the independent variable on a dependent variable. Partial mediation, in contrast, happens if there is both a significant indirect effect and a significant direct effect of the independent variable on a dependent variable after controlling for the mediator (Rucker, Preacher, 
Tormala, \& Petty, 2011). The results of this study clearly show both significant direct and indirect effects of emotional arousal on message compliance. This finding indicates that the coping response has a partial mediating effect on the relationship between guilt/shame arousals and health message compliance. The results of this study also indicate that the direct effect of emotional arousal on the two exogenous/dependent variables - coping response and message compliance - varies. Specifically, for shame, the emotional arousal has a stronger effect on the coping response $(\beta=.38)$ than message compliance $(\beta=.24)$, but for guilt, conversely, the emotional arousal has a weaker effect on the coping response $(\beta=.31)$ than message compliance $(\beta=.36)$. The total effect of emotional arousal towards message compliance is .45 for guilt, and .36 for shame (see Figure 6.1). This finding provides empirical evidence for the literature of emotion and health communications, where guilt or shame arousal has been conceptualised as a factor contributing to an understanding of health message persuasion (Agrawal \& Duhachek, 2010; Duhachek et al., 2012).
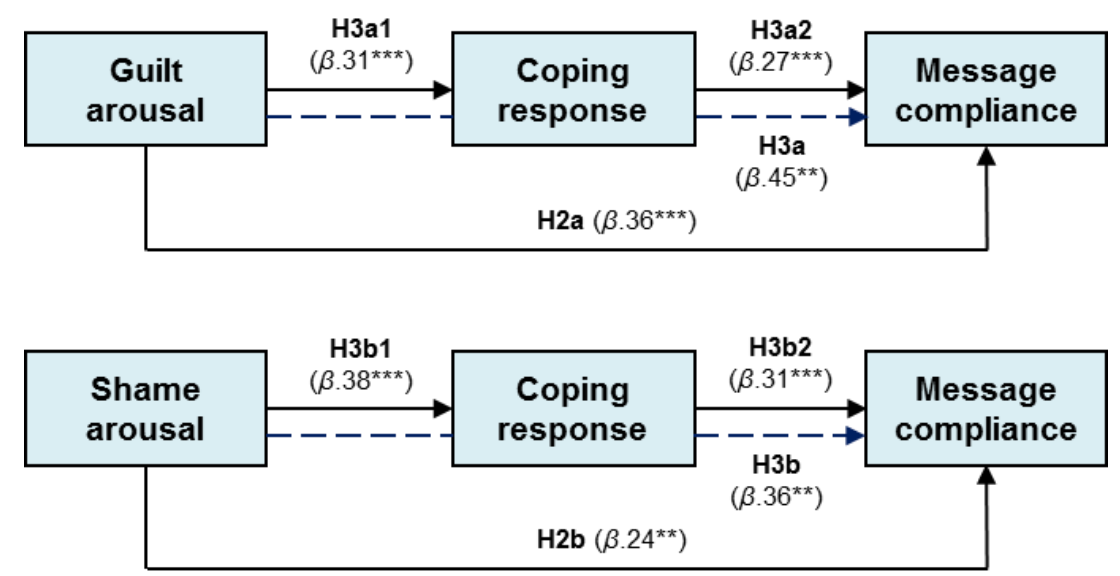

Note $: \longrightarrow$ : significant direct relationship, $\longrightarrow$ : significant indirect relationship; $* * p<.01, * * * p<.001$.

Figure 6.1. Direct and Indirect Relationships between Emotional Arousal and Message Compliance

In the literature, guilt and shame have demonstrated differential effects in encouraging positive behaviours. Guilt tends to create more favourable behavioural responses while shame is more likely to trigger negative behaviours (Abe, 2004; Lindsay-Hartz, 1984; Orth et al., 2010). But the partial mediating effect of the coping response, evidenced in this study, indicates that shame can more successfully influence health message compliance in the presence of adaptive coping responses. The implication is that neither 
emotional arousal nor adaptive coping response is sufficient on its own to motivate people to comply with the health message; rather, the two working in tandem provide the greatest potential value in the health message persuasion.

The mediating effect of coping responses can be explained by protection motivation (Rogers, 1983; Tanner et al., 1991). That is, threat appraisal results in protection motivation and then this motivation triggers an individual's coping response. Threat appraisal, in the case of guilt and shame, is the judgement made by individuals that they are exposed to a given danger to personal notions of self-integrity. Protection motivation is perceived to be an intervening factor that has been able to arouse, sustain, and direct the activity of an individual (Rogers, 1983). The stimulation of protection motivation can manifest itself in an adaptive or maladaptive coping response (type of coping) from an individual and varies the likelihood of health message compliance. There is evidence that adaptive coping responses are related to the removal of a threat, whereas maladaptive coping responses are related to the control of a fear in response to the threat (Tanner et al. 1991). Specifically, an adaptive coping response occurs when a fear arousal stimulates message receivers to recognise a fear and search for ways to deal with the threat (Schoenbachler \& Whittler, 1996). For example, below is an adaptive coping response to this study's binge drinking stimulus ad:

"Felt very in your face. Made me wonder about the consequences of alcohol and binge drinking." (Respondent 52, European New Zealander, male, 18 years old)

In comparison, a maladaptive coping response happens when a fear arousal stimulates message receivers to find a way to avoid the notion of threat instead of removing a threat (Schoenbachler \& Whittler, 1996). This maladaptive coping response includes avoidance or denial of the threat or the impending issue. For instance, maladaptive responses in this study's binge drinking stimulus ad are as follows:

"Binge drinking too deep in New Zealand culture to be persuaded by a subjective poster like that, as young people are not shamed by it, and do not shame their peers." (Respondent 64, European New Zealander, male, 20 years old)

"It made me feel unhappy that it was so heavily shaming and blaming young women without taking into consideration the reasons for drinking, i.e., student culture or personal reasons." (Respondent 159, European New Zealander, female, 19 years old) 
Understanding the coping response to emotional arousal that results from an emotional appeal is of interest because it can impact on the cognition and motivation process (Dickinson \& Holmes, 2008; Dickinson \& Holmes, 2011). For individuals who have a maladaptive response, it is likely that they will reject the message being communicated by health communicators while an adaptive coping response is likely to result in message acceptance, which is desirable to health promoters. Understanding how guilt or shame emotional arousal can impact an individual's coping response is thus significant because of the relationship between a coping response and compliance with the health message whereby health communicators seek to trigger adaptive coping responses when using guilt or shame appeal in communications.

\subsubsection{Moderating Effect of Personal Cultural Orientation}

The study found full support for the set of hypotheses (H10, H11) which propose that personal cultural orientation moderates the effect of shame emotional arousals on message compliance, but do not moderate the effect of guilt emotional arousals on message compliance. In addition, no differences were found in message compliance levels between individualist and collectivist orientations, regardless of either guilt or shame type. In other words, despite the findings that there was no difference in the levels of message compliance between individuals with different personal cultural orientation, there was a difference in the predictive power of guilt versus shame arousals on message compliance. That is, guilt predicted message compliance in both orientations, whereas shame only predicted message compliance in collectivist people. This is consistent with previous predictions that guilt is more favourable for behavioural responses than shame (Abe, 2004; Ghorbani, Liao, Çayköylü, \& Chand, 2013; Lindsay-Hartz, 1984). For individualist-oriented people, when deciding on the level of compliance with the health message, shame was not a determinant of the amount. On the contrary, when deciding on the level of message compliance, guilt was a significant predictor of message compliance.

Shame was not predictive of individualist-oriented people's compliance, which is consistent with the literature on shame. Bagozzi et al. (2003) compared Dutch (i.e., national cultural individualism) and Filipino (i.e., national cultural collectivism) salespersons' experiences of shame as a consequence of customer actions. The real experience of shame was very similar for both groups (i.e., painful, self-focused emotion, the core self was felt to be threatened), but the behavioural reactions were different. For 
Dutch employees, shame diminished customer relationship-building, whereas for Filipino employees, shame enhanced it. The link between shame and defensive reactions, such as running away and hiding from others (Tangney et al., 2007; Tangney et al., 1992), is typical of individualist cultures (Kitayama et al., 1995), where the sense of self as independent is valued and shame can be interpreted as a sign of weakness (SilfverKuhalampi, 2009). From this viewpoint, hiding shame is a reasonable thing to do for individualists, but defending the self against shame in this way is probably seen as inadequate for collectivists. This might explain why shame does not predict message compliance for individualist individuals.
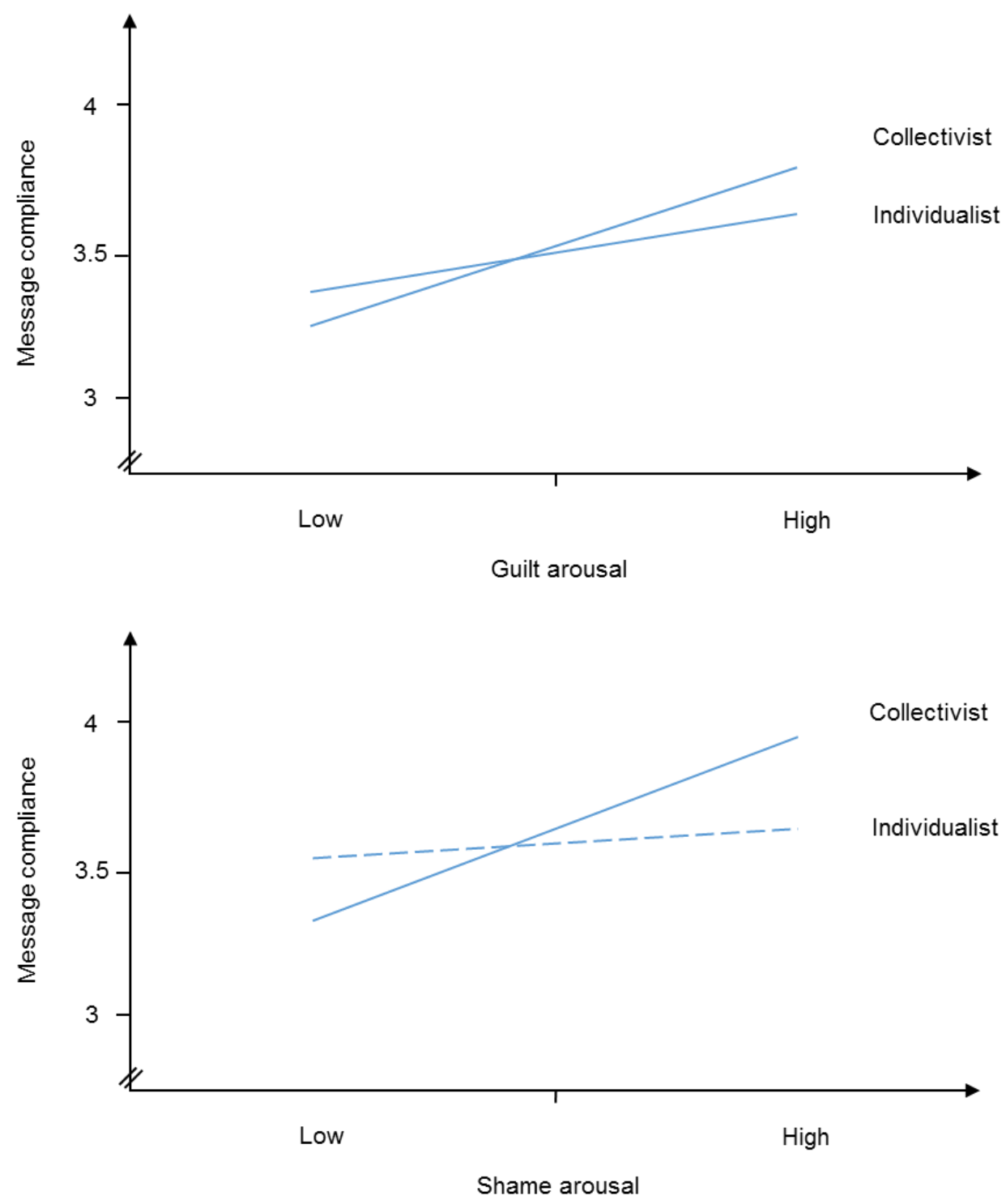

Note: — : significance, $--:$ no significance.

Figure 6.2. Regression of Guilt and Shame Arousal on Message Compliance between Personal Cultural Orientations 
This study found the interactive effects of the emotion type and personal cultural orientation on message compliance (see Figure 6.2). That is, the impact of shame arousal on message compliance is moderated by personal cultural orientation. Specifically, there is a significant direct positive influence of emotional arousal on health message compliance for collectivist individuals, but not for individualist individuals experiencing shame. On the other hand, the study does not find any moderating effect of personal cultural orientation on the relationship between guilt arousal and message compliance. This shows that there is a significant direct positive influence of emotional arousal on health message compliance for both individualist and collectivist individuals experiencing guilt.

From the national-level point of view, the findings are consistent with previous crossculture studies. For shame, some authors believe that coping with the emotion depends on the cultural concept of self, which is different across cultures (Bagozzi et al., 2003; Dean \& Fles, 2016; Fontaine, Poortinga, Setiadi, \& Markam, 2002; Kitayama et al., 1995; Mesquita \& Karasawa, 2004; Wallbott \& Scherer, 1995). They suggest that in the collectivist cultures, showing shame to others is seen as courageous and positive, because it is an essential part of social interaction. In empirical studies, Wallbott and Scherer (1995) found extensive evidence of cultural differences in shame experience across 37 countries. In collectivist societies, shame is a rather acute, short-lived emotional experience and is seen as having fewer negative influences on self-image and on social relationships compared to individualist societies. This difference is probably due to differences in coping responses with shame. Individuals in collectivist cultures seem to be more able to cope with shame adaptively. Collectivist people see their sense of self depending on social relationships, and the only possibility for recovering the positive sense of self is to repair their relationships. By contrast, individuals in individualist cultures are more likely to react in maladaptive ways which harm their interpersonal relationships. This is possibly because an easier way to relieve shame for individualist people is to avoid others or shift the blame to someone else rather than take responsibility for the matter (Silfver, 2007; Sznycer et al., 2016).

\subsubsection{Moderation Effect of Distinct Emotion Type}

This study found no significant difference in the effect of the emotion type on message compliance. Specifically, the results of SEM multigroup analysis for emotional arousal to 
message compliance show that the total effect, including the direct and indirect effects via coping responses was .45 for guilt and .36 for shame (see Figure 6.1). However, the difference is not significant across guilt and shame arousals. This means guilt arousal and shame arousal are equal in predicting message compliance.

The finding is inconsistent with studies within the psychology literature which suggest that guilt and shame are distinct in supporting guilt with more subsequent approach intentions than shame in terms of consequential outcomes (Orth et al., 2010; Wong \& Tsai, 2007). In the present study, as shown in Section 6.2.5, coping responses are found to be the mechanism through which guilt and shame arousals lead to compliance with emotional appeal messages. The presence of the mediation effect of coping responses is one of the reasons explaining the inconsistency. The study's finding suggests that individuals who experience self-conscious emotional arousal from health emotional appeal communications are likely to comply with the persuasive message regardless of guilt or shame. However, as shown in Section 6.2.6, personal cultural orientation moderates the effect of emotional arousals on message compliance across guilt and shame. That is, guilt arousals predicted message compliance in all personal cultural orientations, while shame arousals only predicted message compliance in collectivist individuals, not in individualist counterparts.

Taken together, it can be seen that when shame is felt, only collectivist people are likely to respond adaptively to the emotion and ultimately comply with the persuasive message, but when guilt is felt, both collectivist and individualist people are likely to respond adaptively to the emotion and ultimately comply with the message. Further, regardless of either guilt or shame, collectivist or individualist, when self-conscious emotion is felt, individuals are likely to respond adaptively to the emotion and ultimately comply with the persuasive message. These findings are consistent with those found in the moral emotions and across cultures literature (Dean \& Fles, 2016; Kim \& Johnson, 2013).

\subsection{Moderating Effects of Regulatory Focus and Self-Construal}

\subsubsection{Moderating Effect of Regulatory Focus on Guilt/Shame Arousal}

The findings of the study indicate the marginal moderation effect of regulatory focus in the relationship between emotional appeals and emotional arousals. In particular, the study found a marginally significant difference in the relationship of shame appeals and 
shame arousals between promotion- and prevention-focused individuals, but no significant difference was found in the relationship of guilt appeals and guilt arousals across regulatory foci. More specifically, the relationship between guilt appeal and guilt arousal is equally strong for both promotion- and prevention-focused individuals. On the other hand, the relationship between shame appeal and shame arousal is stronger for prevention-focused than for promotion-focused individuals. This implies that regulatory focus moderates the relationship between emotional appeals and emotional arousals for shame. In contrast, regulatory focus does not moderate the relationship between emotional appeals and emotional arousals for guilt.

The results are evidence of the moderating effect of regulatory focus for the premise that shame is a better predictor for emotional arousal level for prevention-focused than for promotion-focused individuals. The findings of the study are fully consistent with the regulatory focus theory (Higgins, 1997, 1998), which highlighted substantial regulatory focus differences in cognition and affect among different individuals (Aaker \& Lee, 2001; Lee \& Aaker, 2002, 2004). Among previous limited studies examining regulatory focus as an individual difference variable (e.g., viewers' regulatory focus), it has been found that there are distinct regulatory fit differences between promotion- and preventionfocused individuals in message persuasiveness (Lockwood et al., 2002; Zhao \& Pechmann, 2007). In studies on anti-smoking messages among adolescents, Zhao and Pechmann (2007) found that for promotion-focused viewers, a promotion-focused positively framed anti-smoking message is the most effective at persuading them not to smoke. For prevention-focused adolescents, a prevention-focused negatively framed antismoking message is the most effective. The current study consistently found that regulatory focus has a stronger effect on the level of negative self-conscious emotional arousals of prevention-focused individuals, compared with promotion-focused individuals for shame. This is because prevention-focused individuals are more motivated to avoid threats to their notions of self-integrity caused by shame, while promotion-focused individuals are less motivated by these threats.

However, differences were found in terms of emotional appeal and its relationship with emotional arousal for shame only. For guilt, guilt appeal is an equally better predictor for emotional arousal level for both promotion- and prevention-focused individuals. It is noted that guilt, in this study, is anticipatory and could be felt less negatively than the 
reactive guilt experienced after engaging in guilt-inducing appeals (Giner-Sorolla, 2001; LaBarge \& Godek, 2006). Thus, manipulative intent is not salient where appraisals do not differ across regulatory foci (Kirmani \& Zhu, 2007). In other words, the insufficient manipulative intent in the anticipatory guilt appeals make promotion-focused people react similarly to prevention-focused people. This finding supports the previous research that regulatory focus is likely to have various moderating effects on individuals' cognition and affect (Cropanzano, Paddock, Rupp, Bagger, \& Baldwin, 2008; Zhao \& Pechmann, 2007).

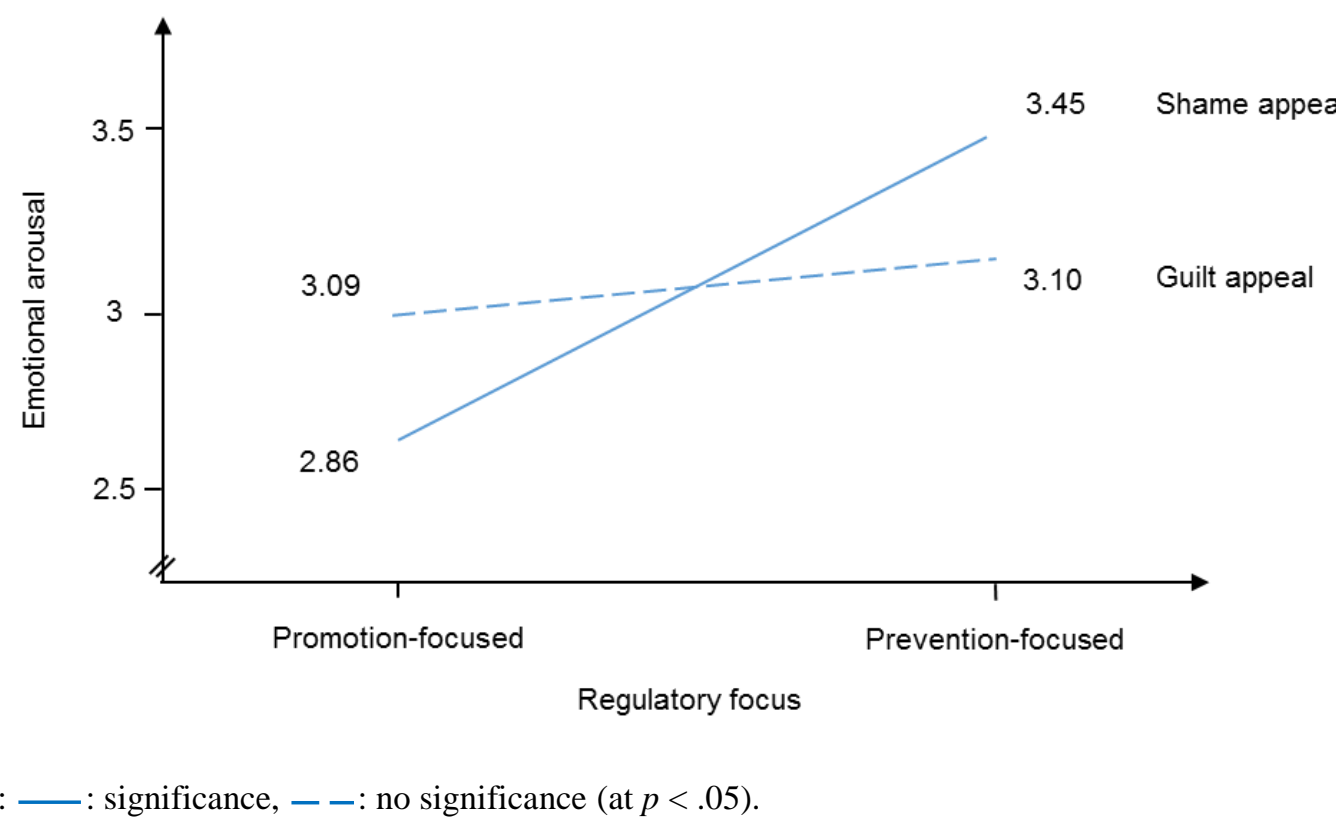

\section{Figure 6.3. Effect of Regulatory Focus and Type of Emotion on Emotional Arousal}

This study tested and found marginal support for the proposition that regulatory focus moderates the effect of guilt/shame emotional appeals on levels of respective guilt/shame emotional arousals. There was a difference in the predictive power of shame appeals on shame arousal intensity level. Specifically, shame is a better predictor in emotional arousal for prevention-focused than promotion-focused individuals, whereas guilt is an equally better predictor in emotional arousal for both promotion- and prevention-focused individuals. Importantly, there are differences in shame arousal intensity levels for individuals with a promotion focus versus a prevention focus $(\mathrm{H} 4 \mathrm{~b})$. That is, preventionfocused individuals exhibit more shame arousal than their promotion-focused counterparts (see Figure 6.3). 


\subsubsection{Moderating Effect of Self-Construal on Guilt/Shame Arousal}

Self-construal also marginally moderates the relationship between emotional appeals and emotional arousals. This finding broadly supports the work of other studies in this area which found that there are various groups of self-construals with differing appraisals (Block, 2005; Martin et al., 2013; Park et al., 2011). The current study finds that the relationship between guilt appeals and guilt arousals for independent self-construals is marginally stronger than for interdependent self-construals. However, the relationship between shame appeals and shame arousals is equally strong for both self-construals. The findings suggest that self-construal does have some impact on the relationship between emotional appeals and emotional arousals. This provides an insight when comparing the moderating effect of self-construal between guilt versus shame emotional types and contrasting this finding with the moderation effects towards emotional responses in general. This indicates that, although guilt and shame are self-conscious emotions, when comparing guilt and shame in terms of distinct emotion, not all self-conscious emotions are equally appraised by customers (Han et al., 2014). These findings provide insight into when and why guilt and shame have different effects on message persuasion.

As a dynamic individual characteristic, self-construal reflects how individuals perceive the self in relation to, and as distinct from, others (Markus \& Kitayama, 1991; Singelis \& Sharkey, 1995). The result of this study demonstrates that self-construals are different in terms of the psychographic factor (the self) and help to explain their tendency towards different self-conscious emotions. The differences between groups of self-construal viewers have long been recognised as opportunities in healthcare marketing messages by social marketers (Evans \& McCormack, 2008; Maibach et al., 2007). Although individuals experience guilt and shame self-conscious emotions, they do so based on a distinct psychologically activated mind-set (Han et al., 2014). The ability to understand the specific characteristics of different self-construal individuals would enable health marketers and public policy makers to incorporate these two self-conscious emotions into health emotional appeal messages to enhance persuasion. This study finds that guilt appeals have a stronger positive impact on guilt arousals for independent self-construals than interdependent self-construals. These results indicate that guilt appeals stimulate guilt arousals to the independent self-construals over the interdependent self-construals. Where personal responsibility/accountability in guilt is more salient, the independents are motivated to promote self-responsibility that foster the feelings of guilt. This belief has 
shaped a moderating effect of self-construal on moral judgements and suggests that independents were more likely to be influenced by the feeling of ego-focused (e.g., guilt, pride) than other-focused emotions (e.g., shame, anger) (Kim \& Johnson, 2014).

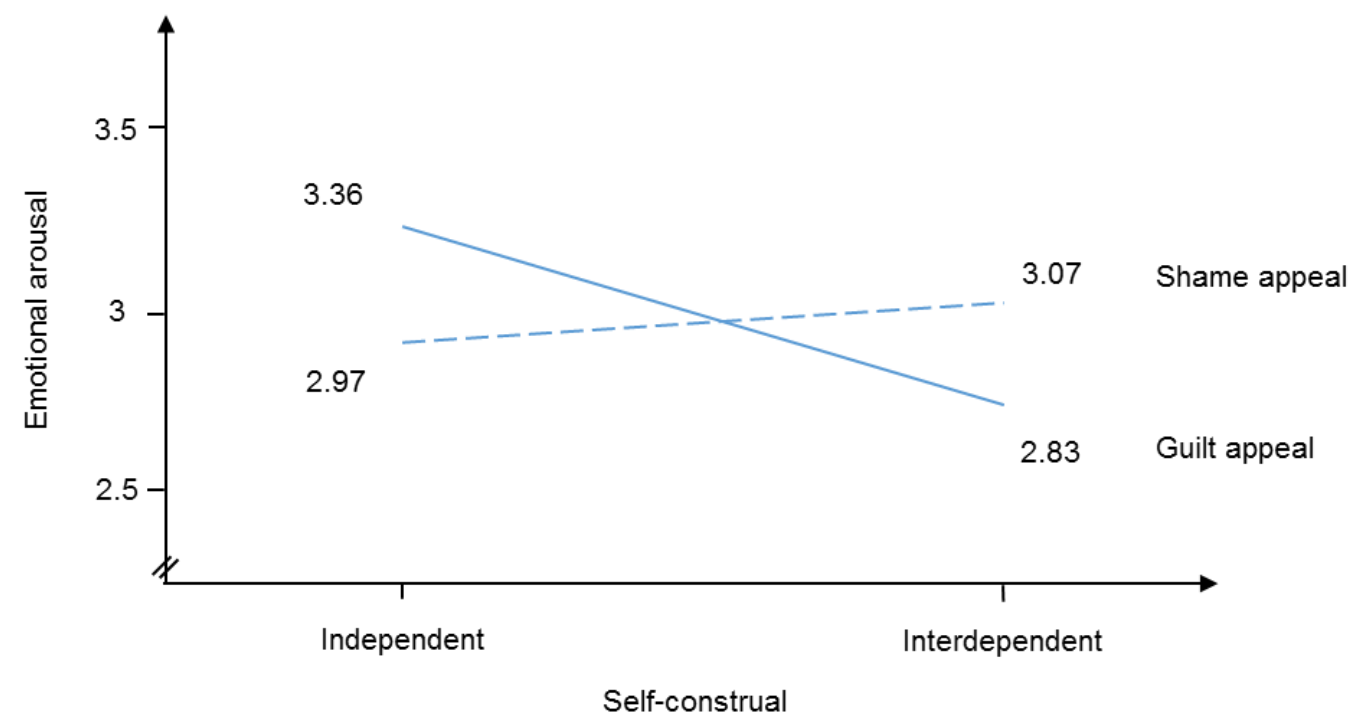

Note: ——: significance, $\_-$: no significance (at $p<.05$ ).

\section{Figure 6.4. Effect of Self-Construal and Type of Emotion on Emotional Arousal}

The findings also indicate that in terms of shame arousals, based on shame appeals, selfconstrual has no mediation. For shame, emotional appeal is an equally better predictor of the emotional arousal level for both self-construals. Consistent with the previous few studies that have directly measured self-construal, this result provides support for the premise that individuals high in interdependent self-construals, for whom social relationships are central to definitions of the self, would express more affective consequences in interpersonal contexts (e.g., shame) (Cross et al., 2010). Interestingly, independent self-construals are also found to be a better predictor of socially disengaging emotions (e.g., shame, anger) like interdependent self-construals (Xie, Leong, \& Feng, 2008). There is, thus, a common misconception that Westerners are not found to selfreport high levels of shame, as independent self-construals are often associated with decreased levels of general anger and social anxiety (Hardin, Varghese, Tran, \& Carlson, 2006; Kim, Kasser, \& Lee, 2003; Xie et al., 2008). Although independence and interdependence differ, there is disagreement over whether they are, in fact, emotionally the same related to specific affective consequences (Kitayama, Snibbe, Markus, \& Suzuki, 2004). These misconceptions have emphasised self-construal differences in 
emotional feelings which, in turn, could deter independent self-construals from complying with shame appeal health messages.

Despite strong interest in cultural differences in emotion, little research has actually measured self-construal to investigate directly how independence and interdependence relate to various guilt and shame emotions. This study tested and found marginal support for the proposition which proposes that self-construal moderates the effect of guilt/shame emotional appeals on respective guilt/shame emotional arousals. There was a difference in the predictive power of guilt appeals on guilt arousal intensity level. Specifically, guilt is a better predictor in emotional arousal for independent self-construals, whereas shame is equally predictive in emotional arousal for both self-construals. Importantly, differences were found in guilt arousal intensity levels for independent versus interdependent self-construals (H5a). Specifically, independent self-construals exhibit significantly higher guilt arousal than their interdependent counterparts, regardless of self-referencing and sources of evaluation (see Figure 6.4).

\subsubsection{Interactive Effects of Self-Construal with Self-Referencing, Sources of Evaluation}

While the main effect of self-construal exists between the relationship of emotional appeal and its emotional arousal, this study did not find any interactive effects of selfconstrual with self-referencing or the sources of evaluation. It found that independent self-construals exhibit significantly higher guilt arousals than interdependent selfconstruals when exposed to guilt appeals. However, both self-construals exhibit equal shame arousals when exposed to shame appeals, regardless of self-referencing and the sources of evaluation (see Section 6.3.2). Unexpectedly, across type of appeal (selfreferenced versus other-referenced guilt and shame, internal versus external guilt/shame), guilt/shame arousals do not respectively differ significantly.

Although self-referencing and the sources of evaluation have been shown to interact significantly with self-construal in marketing and psychology studies investigating emotional appeals and message persuasion (e.g., Block, 2005; Wong \& Tsai, 2007), this was not the case in this study. Levels of emotional arousal elicited from an emotional appeal were not impacted by the interaction of self-construal with self-referencing (rejected H6a for guilt, H8a for shame) or with the sources of evaluation (rejected H7a for guilt, H9a for shame). They were found to be nonsignificant, indicating that self-construal 
does not interact with self-referencing or the sources of evaluation to affect the emotional arousal levels. This is somewhat counter intuitive. One would expect that, for independent self-construals, levels of guilt arousal elicited from a self-referenced guilt appeal are higher than those from an other-referenced guilt appeal (H6a), and levels of guilt arousal from an internal guilt appeal are higher than those from an external guilt appeal (H8a). Moreover, levels of shame arousal elicited from an other-referenced shame appeal are higher than those from a self-referenced shame appeal $(\mathrm{H} 7 \mathrm{a})$, and levels of shame arousal from an internal shame appeal are higher than those from an external shame appeal (H9a). Conversely, for interdependent self-construals, levels of guilt/shame arousal elicited from a guilt/shame appeal are not impacted by self-referencing and the evaluation source (H6b, H7b, H8b, and H9b accepted). One interpretation of this result is that the message's attributes of self-reference or internal source of evaluation based on focus towards the self (versus the others) or orientation to one's own standards/views (versus the others') are not salient to individuals with an independent view when processing the message. This is because independent individuals have sub-types (e.g., relational versus independent self-construal) that may vary effects on cognition, affect, and motivation (Cross et al., 2010).

Indeed, such sub-types of independent self-construal have been found to result in the interactive effect with self-referencing (Cross, Morris, \& Gore, 2002; Martin et al., 2013). Martin et al. (2013), when examining how a consumer's relational self-construal and selfreferencing influence their evaluations and behavioural intention regarding binge drinking and speeding/road safety ads, found that in the relational self-construal, the self is defined largely in terms of close relationships, resulting in a variation in self-referencing processes. That is, high relational self-construal consumers are more persuaded by ads featuring dyadic models (i.e., other-reference), while low relational self-construal participants prefer ads featuring solitary models (i.e., self-reference). The findings suggest that attitudes are most favourable when relational self-construal and the self-referencing shown in the message are congruent.

The findings of the current study do not support the previous research which suggests that self-construal interacts with self-referencing to impact on self-conscious emotional appeals processing (Aaker \& Williams, 1998; Block, 2005). For instance, Aaker and Williams (1998) examined the persuasive effects of ego-focused (e.g., pride) versus 
other-focused (e.g., empathy) emotional appeals across cultures and found that the selfreference effect was obtained for independent self-construals who viewed a pride emotional appeal. More specifically, Block's (2005) studies on fear and guilt appeals suggested that for individuals with independent construals, the self-reference compared to the other-reference effect is either advantageous or disadvantageous depending on the types of emotions (e.g., fear versus guilt). That is, for people who hold a predominantly independent self-construal, superiority of self-referencing over otherreferencing holds for guilt appeals but not for fear. The discussed discrepancy could possibly be due to the measure of self-construals. In the aforementioned studies, the selfconstruals were measured by ethnic-level characteristics (American versus Asian), while in the present study, they were measured by individual-level characteristics (independent versus interdependent individuals).

Past research has shown that individuals' self-construal is dominated by culture. That is, an independent self-construal is dominated by Western societies and an interdependent self-construal is dominated by Eastern societies (Markus \& Kitayama, 1991). However, more recent studies that measure cultural self at the individual level encounter the scale validity issue (Levine et al., 2003; Lu \& Gilmour, 2007). Levine et al. (2003) found that in contrast to the theoretical assumptions of cultural self-construals, people in the Asian contexts (i.e., Japanese, Korean) did not score significantly higher on the interdependent self-construal scale. Thus, it is possible that the interactive effects of self-construal with self-referencing or the sources of evaluation on cognition and affect vary due to the construct's conceptual level difference: cultural versus psychological mechanism (Datu, 2015; Dean \& Fles, 2016). According to Dean and Fles (2016), within-culture (psychological level) distinct self-construals reveal their corresponding attributional and motivational processes. Therefore, it can be seen that the interactive effects of independent self-construals with self-reference or internal source of guilt and shame exist only if comparing self-construal between cultures (cultural level). Although the discussed findings are inconsistent with the extant studies evaluating the interactive effects of selfconstrual with self-referencing (Block, 2005) or the sources of evaluation (Wong \& Tsai, 2007), this study confirms the literature that indicates both cultural and psychological dimensions are essential in establishing the valid self-construal construct (Datu, 2015). In terms of psychology, relational (versus collective) self-construal relates to a person's beliefs about the degree to which they define themselves in terms of their close 
relationships (e.g., close friend, spouse) rather than a collective relationship with generalised others (e.g., people of the same ethnicity) (Cross et al., 2010). Consequently, relational self-construal is suited to studies of individuals with an independence, who are more likely to include individual relationships (e.g., best friend) in their sense of self than more general in-groups.

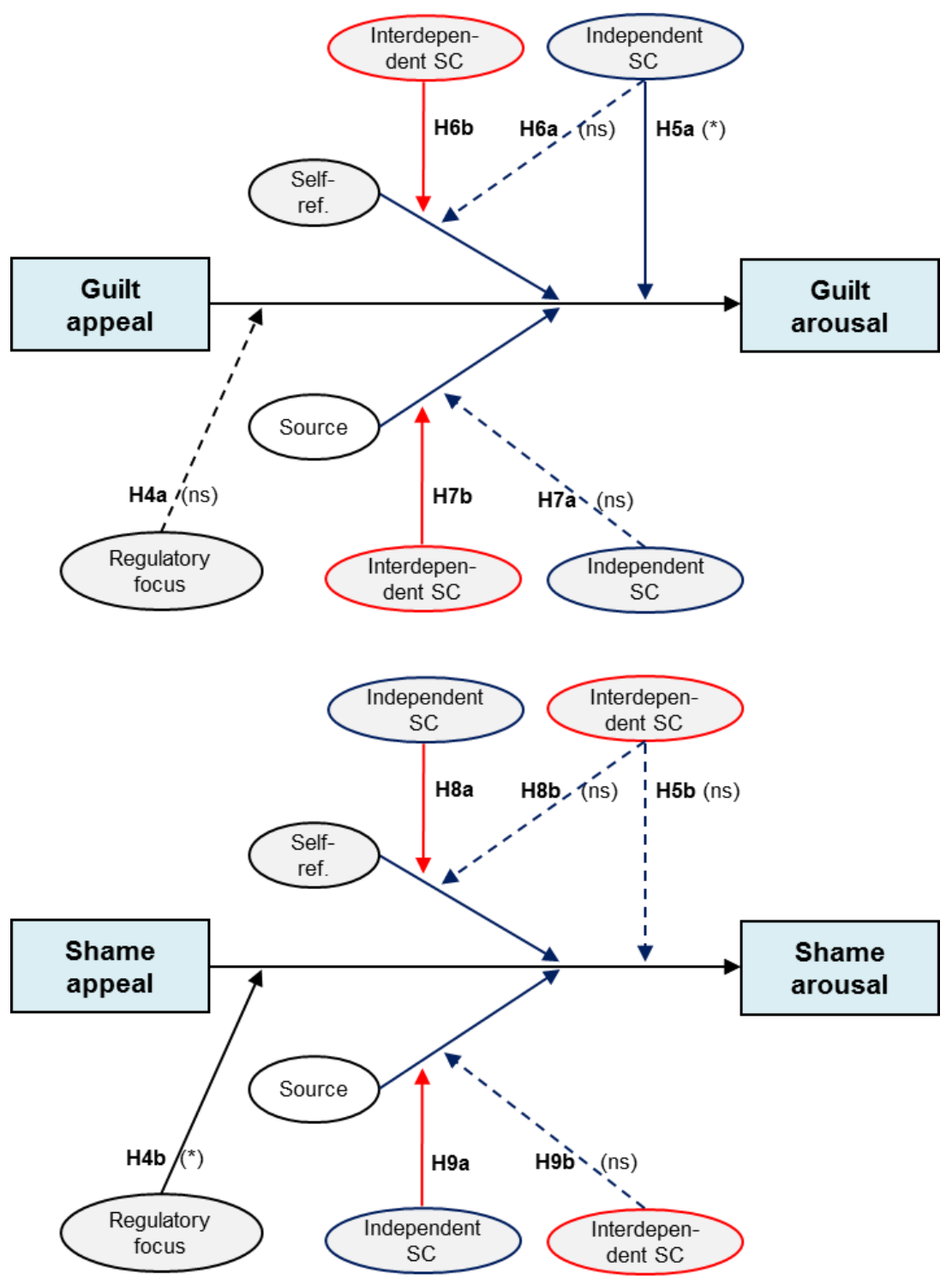

Note: $\longrightarrow$ : significant relationship, $\rightarrow-\rightarrow$ : insignificant effect; ns: no significance, $* p<.05$.

Figure 6.5. Moderating Effects of Regulatory Focus and Self-Construal on Emotional Arousal

Figure 6.5 summarises the findings of the moderating effects of regulatory focus and selfconstrual on guilt versus shame arousal discussed in the current study so far. 


\subsection{Further Dependent and Demographic Factors}

\subsubsection{Further Dependent Measures}

The study confirms that message compliance is associated with both attitudes towards the ad and the health message, and binge drinking intentions. Consistent with the literature (e.g., Johnston \& White, 2003), this research found that there was a medium negative correlation between message compliance and binge drinking intentions, with higher levels of compliance with the message associated with lower levels of intention to binge drinking. Large positive correlations between binge drinking intentions and attitudes towards the ad and the health message were also found, with higher levels of attitude towards the ad as well as the message associated with higher levels of compliance with the health message.

The results reveal the predicted pattern regarding the correlations between the three separate dependent measures: attitude, compliance, and intention. Together, they provide convergent evidence in support of the proposition that individuals experiencing guilt or shame from anti-binge drinking communications are likely to comply with the pro-health message and, ultimately, change binge drinking behaviour. Specifically, the higher the emotional arousals, the greater the attitudes towards the ad and the health message featured in the ad, the greater the health message compliance, and the lower the intentions to binge drink.

\subsubsection{Demographics}

The results show the moderating effect of gender in the relationships between emotional appeal and its arousal, emotional arousal and coping response, and emotional arousal and message compliance (except for the relationship between coping response and message compliance). The strength of these relationships was significantly greater for males than for females, indicating that guilt and shame appeals had stronger predictive effects on compliance with the message by male than by female viewers. This can be explained by the fact that females appeared to be less concerned about their drinking as they consumed less than their male counterparts (Hutton, 2012). This fact is confirmed by the characteristics of respondents in the current study female students drank less heavily than male ones (see Table 5.2). 
In terms of alcohol consumption, the results have shown there is no moderating effect on the paths of emotional appeal $\rightarrow$ emotional arousal $\rightarrow$ coping response $\rightarrow$ message compliance. However, the results of ANCOVA indicate that there was a difference in the message compliance score between individuals with low-risk levels and high-risk levels of alcohol use. Specifically, individuals with low-risk levels of alcohol use showed greater compliance with the anti-drinking message than those with high-risk levels. This finding is consistent with previous studies which found that individuals with different risk levels of alcohol use respond differently to anti-drinking messages and that high-risk drinkers are likely to be more resistant to messages suggesting change (Logan et al., 2010).

\subsection{Overall Effects}

The findings of correlational analyses revealed linear relationships between emotional appeal, its arousal, coping response and message compliance. All independent and dependent variables were positively correlated with each other. Importantly, emotional appeal had a strong positive correlation with emotional arousal (see Table 6.1).

Table 6.1. Zero-Order Pearson Correlations for Key Variables

\begin{tabular}{lcccc}
\hline & EAp & EAr & CR & MC \\
\hline Emotional appeal & - & & & \\
Emotional arousal & $.547^{* * *}$ & - & & \\
Coping response & $.398^{* * *}$ & $.325^{\star * *}$ & - & \\
Message compliance & $.335^{* * *}$ & $.352^{* * *}$ & $.371^{* * *}$ & - \\
\hline
\end{tabular}

$n=266 ; * * * p<.001$.

To assess the predictive effects of moderators on mediators, emotional appeal, emotional arousal, coping response, and message compliance were regressed to groups of regulatory focus, self-construal, and personal cultural orientation (see Table 6.2). Generally, results revealed that emotional appeal positively predicted emotional arousal and emotional arousal positively predicted message compliance. These imply that higher levels of emotional appeal could be associated with higher emotional arousal and higher emotional arousal is associated with greater message compliance. It is notable, however, that prevention-focused, independent, and collectivist people had stronger predictive effects on the abovementioned mediating variables across guilt and shame. Specifically, 
prevention-focus had stronger predictive effects on shame arousal, independence had stronger predictive effects on guilt arousal, and collectivism had significantly predictive effects on compliance with shame appeal messages compared with its counterparts.

Table 6.2. Path Coefficients across Moderators

\begin{tabular}{|c|c|c|c|c|c|c|}
\hline \multirow{2}{*}{ Emotion } & \multicolumn{4}{|c|}{$\mathrm{EAp} \rightarrow \mathrm{EAr}$} & \multicolumn{2}{|c|}{$\mathrm{EAr} \rightarrow \mathrm{MC}$} \\
\hline & ProRF & PreRF & IndSC & IntSC & IndPCO & ColPCO \\
\hline Guilt & $.563^{\star \star \star}$ & $.507^{\star \star \star}$ & $.614^{\mathrm{a} * \star *}$ & $.502^{b * * \star}$ & $.263^{\star \star *}$ & $.354^{\star * \star}$ \\
\hline Shame & $.572^{\mathrm{a}^{\star * *}}$ & $.770^{\mathrm{b} * \star *}$ & $.688^{* * *}$ & $.578^{\star * *}$ & ns & $.361^{\star *}$ \\
\hline
\end{tabular}

ns: no significance, ${ }^{* *} p<.01, * * * p<.001 ; \beta$ values in the same row with a different superscript differ marginally significantly from each other at $p<.10$.

Overall, the present study's results supported the set of hypotheses that the degree to which guilt or shame, coping responses and their subsequent message compliance in message receivers are functions of type of emotion appeal, regulatory focus (H4b), selfconstrual (H5a), and personal cultural orientation (H11) of individuals. The more prevention focused, the more likely individuals experience shame from health message exposure, and are, therefore, more likely to comply with the health message. The more independent self-view, the more likely individuals experience guilt from health message exposure, and are, therefore, more likely to comply with the health message. Moreover, the effect of self-conscious emotions on message compliance varies depending on the type of emotion interacting with personal cultural orientation. When individualist individuals are exposed to the health emotional appeal message, guilt is a predictor of message compliance while shame is not. When collectivist individuals are exposed to the health message, both guilt and shame predict message compliance equally well.

This finding indicates that message viewers could be categorised as either individualists or collectivists based on individual orientations to culture. This type of dyadic categorisation and moderation in shame arousals towards behaviour intentions is consistent with previous studies about collectivist tendencies to motivate reparative actions (Czub, 2013; Silfver, 2007). Shame is caused by violations of character (Lewis, 2000; Van Vliet, 2009) and is related to individuals' identities (Ghorbani et al., 2013). Shamed collectivist individuals try to comply with the threatening health message to reduce their negative emotion feelings and restore their self-image. For collectivists, 
shame can transform threatening message viewers' characters and give them a lower evaluation of themselves (Lindsay-Hartz, 1984).

Shamed individualist people do not defend their images and characters when facing the threatening message to the same extent as collectivist people, since individualist people are expected to highly value the independent self even when it is difficult to do so. In addition, it is hard to imagine negative matters such as losing face when individualist people feel shame. Therefore, complying with the shame appeal message is not likely to change how individualist people perceive the self as independent from others. This could explain why shame is not a significant predictor of individualist people's compliance with the threatening health message. Individualist people might still feel shame to face the threatening message using shame appeal, as indicated by the two sets of hypotheses (H8a and H9a). However, results do not indicate that shame is the reason for individualists to comply with the threatening health message.

In contrast, shamed collectivist people try to protect and restore their own images and characters when facing the threatening message. When collectivist people feel shame and condemn themselves, they still want to protect the self as a dependent entity to keep a positive image. This is because, in collectivist individuals where interdependence is highly valued, defending the self against shame would likely be seen as unreasonable. Demonstrating shame to others is seen as better because it helps to maintain relationships and social cohesion (Silfver-Kuhalampi, 2009; Silfver, 2007; Tangney et al., 2007). So it is important for collectivist people to make reparations in order to restore a positive view of themselves. By complying with the threatening health message, shamed collectivist transgressors send out the message that they take responsibility for their wrongdoings. This positive image can help restore characters that were damaged during transgressions. Therefore, when collectivist people feel shame and think their self-integrity is vulnerable, they need to take actions to protect or improve their image from the threatening health message.

\subsection{Chapter Summary}

In sum, the results of this study present significant contributions to the literature of emotions and health communications, specifically with regards to anti-drinking. The supported hypotheses for the linear relationships among emotional appeal, its arousal, 
coping with emotion, and compliance to the health message indicate that the present study supports the extant literature in self-conscious emotions and pro-social, charitable donation behaviour and further contributes towards it by studying these relationships specifically in the context of health communications, self-conscious guilt and shame emotions.

Moreover, the relationships between each of the three emotion-coping-intention components and the individual difference in self-construal, regulatory focus and personal cultural orientation show that across the groups, individuals process health emotional appeal messages differently in terms of cognition, emotion, motivation, and social behaviour. Although not in agreement with previous studies on self-construal interacting with self-referencing or the sources of evaluation, the set of findings supports past studies of self-construal on its main effect. Furthermore, the present study also found that the regulatory focus and personal cultural orientation of individuals has main effects on their emotional arousal and compliance towards the health emotional appeal messages. Importantly, indirect positive relationships are evident via a coping response (for both categorical and continuous measures). The importance of the coping response to emotional arousal and its compliance towards the message emphasises the role of the coping response, where emotion-motivation relationships (underlying theoretical base for coping response) among individuals are considered significant.

This chapter has interpreted and discussed the results of this present study. The next chapter presents the research contributions and implications that can be drawn from this study. Limitations of the study are also described, followed by a discussion on the recommendations for future research. 


\section{Chapter 7. Contributions, Implications and Conclusion}

\subsection{Introduction}

The results of the present research provide several contributions to theory, research method and practice. The theoretical and methodological contributions are discussed, followed by implications for managerial practice. Limitations, future research directions and conclusion are also discussed.

\subsection{Theoretical Contributions}

The development and empirical testing of the effectiveness of guilt versus shame appeals on the health communications model - bringing coping responses to self-conscious emotion as a psychological construct and health communications area together - is a key contribution of this study. The effectiveness of guilt versus shame appeals on the health communications model establishes clear links between: $(i)$ the degree of emotional appeals and emotional arousals, (ii) emotional arousals and health message compliance, and (iii) the mediating effect of coping responses in the relationship between emotional arousals and health message compliance. Further, the model also forms (iv) the moderating effects of individuals' self-construal, regulatory focus and personal cultural orientation.

Although guilt and shame can clearly play a role in consumer motivation, few studies in the literature have examined their appeals' impact (e.g., Agrawal \& Duhachek, 2010; Boudewyns et al., 2013; Duhachek et al., 2012). It is important that this research examined guilt and shame separately through specific emotional arousals rather than appeals to control unintentional emotions, other than guilt or shame, evoked by the appeals to more fully understand the linkage between distinct emotion and behaviour (Carey et al., 2013). This is because researchers have recognised that the interplay between the different emotions can determine the message effectiveness (De Pelsmacker et al., 2011; Dillard \& Nabi, 2006). By examining the distinction of guilt versus shame arousals and their respective correlates with coping responses or behaviour intentions, this study's findings could be generalised and applied directly to other stimulus materials (e.g., video, visual) and other contexts (e.g., pro-social behaviour). 
The construct of coping response has become more significant in emotion literature. Although a considerable body of extant literature focuses on the coping response to emotion and its influence on various aspects of persuasive message acceptance (e.g., smoking message, Dickinson \& Holmes, 2008), there is limited research incorporating the role of coping response, as a psychological response to self-conscious emotional arousals, with pro-health or pro-social behaviour. Researchers have mostly emphasised other underlying factors as important predictors of certain behavioural intentions. For example, persuasion knowledge: ad credibility, perception of manipulative intent (Coulter et al., 1999; Hibbert et al., 2007; Lwin \& Phau, 2010), and anger (Coulter \& Pinto, 1995); agent knowledge: beliefs about the characteristics, competencies, and goals of the persuasion agent (e.g., the charity, Hibbert et al., 2007); perceived threats to unknown others, response-efficacy and self-efficacy (Lindsey, 2005); and responsibility (Basil et al., 2001, 2006).

The coping response plays a vital role in understanding the effects of negative emotional arousals on persuasion. The impact of coping responses, especially to self-conscious guilt and shame emotions on threatening health-related behaviour has been studied only minimally in health communications literature, although people's intentions and behaviours are directly influenced by the psychological aspects of their encounter environment. Although scant and impartially theoretical in nature, the existing literature submits evidence, however, that the efficacy of coping responses is an important factor in the study of the effects of guilt and shame emotional arousals on health message persuasion. The effect of the coping response on behavioural intentions depends on an adaptive or a maladaptive coping. Yet, the role of the coping response as a variable in the guilt/shame arousals-message compliance model is still not well established. Only limited health communications research on the fear-persuasion model has pointed to the significant effects of coping response variables - mainly, the type of coping response - on some aspects of persuasion, such as message acceptance (e.g., Dickinson \& Holmes, 2008).

In the specific self-conscious guilt and shame emotions, these effects are even more prominent. The scarcity of studies on distinct guilt and shame emotions and the relationship between coping responses to guilt/shame arousals and health message compliance increases this study's potential contribution to the literature of self-conscious 
emotions and health communications. The present study was undertaken with the intent to contribute to the extant literature on health communications using self-conscious emotions by investigating the mediating effect of the coping response as a psychological response to emotional arousals on persuasive health message compliance. The primary results reported here support the already established understanding and perception of the coping response-behavioural intention relationship. Furthermore, these findings potentially provide some important theoretical contributions to the accumulating theories on cognition, emotion, motivation, and social behaviour.

This study was intended, in part, to contribute to the process of model building in health communications using self-conscious emotions. A model of the effectiveness of guilt versus shame appeals on health communications was developed, based on the theoretical frameworks of cognition, emotion, motivation and empirical research focusing on prosocial and charitable donation messages and persuasion, and tested with data collected through the survey. All the possible causal linkages of the proposed model were empirically tested and the results mostly supported the study's hypotheses. That is, guilt and shame emotional appeals significantly and positively impact the respective guilt and shame emotional arousals from the health message. In turn, guilt and shame arousals significantly influence coping responses and, ultimately, message compliance. The proposed model can be used as a foundation for further research to develop a better understanding of the linkages among underlying factors, and may be employed as a conceptual background to study the effectiveness of guilt and shame appeal messages in pro-health communications.

The results of this study reveal that the coping response has a partial mediating effect on the relationship between guilt/shame arousals and health message compliance (see Figure 3.1 for the conceptual model). The partial mediating effect of the coping response means that emotional arousals influence both coping responses (indirectly) and message compliance (directly). This mediator is significant in explaining the nondifferential effect of distinct guilt versus shame emotions (see Section 6.2.7). In charitable donations literature, it has been established that individuals' behavioural intentions are influenced by a variety of characteristics of guilt appeals (e.g., types of guilt, levels of intensity, Giner-Sorolla, 2001; LaBarge \& Godek, 2006; Renner et al., 2013) and characteristics of message (e.g., message framing, sidedness, Agrawal \& Duhachek, 2010; Duhachek et al., 
2012; Renner et al., 2013). Although the coping response has been accepted as a psychological response (Dickinson \& Holmes, 2008; Schoenbachler \& Whittler, 1996), it has received comparatively limited attention within self-conscious emotional appeal message research. Subsequently, very little is known about how this underlying variable impacts on persuasive message compliance, especially in a health communication context. This study has some fundamental theoretical worth that enhances the understanding of the role of coping response, which is that the coping response is core as a psychology-based predictor of guilt and shame emotional appeal message compliance. There is extensive empirical support for the Cognitive-Motivational-Relational Theory of Emotion (Lazarus, 1991a, 1991b) by many studies in threat/fear and behaviour discipline, but examination based on applying this theory to investigating the behavioural intention of self-conscious guilt and shame emotional appeal health messages appears to be scant. Therefore, this study gives empirical support to the Cognitive-Motivational-Relational Theory of Emotion. Understanding emotion-driven behavioural intentions, as manifested or as influenced by cognition/motivation, can support researchers in developing emotional appropriate mechanisms to better understand the theoretical premise of selfconscious emotional appeal messages.

The current study has uncovered new evidence that adds to the currently limited knowledge of the influence of the coping response on guilt and shame appeal effectiveness. But there is little agreement on which underlying factor most efficiently explains the variation in aspects of message persuasion. This study supports including the coping response variable as a reliable and valid predictor in self-conscious emotional appeal effectiveness. This is one of the main contributions of this study and, up until now, the only literature on this subject. It appears that coping responses generate many differences in persuasive message compliance. This implies that the coping response may serve as a potentially powerful predictor and determinant of major self-conscious emotional appeal messages in such areas as information source, attitude towards message and behavioural change. Thus, researchers should give more attention to the coping response in emotion when studying self-conscious emotional appeals and perhaps other areas of the message process (e.g., coping processes, judgements and decision making).

In addition, the study utilised the Theory of Self-Construals (Markus \& Kitayama, 1991) to identify individuals as independent versus interdependent self-construals in New 
Zealand. The study of self-construals has gained significance in the cultural psychology discipline (Cross et al., 2010), but there is much research comparing self-construals as stable cultural characteristics (Block, 2005; Kim \& Johnson, 2014) rather than dynamic individual characteristics. This study is the first attempt to this end and contributes to laying the groundwork for further analyses on self-construals in New Zealand. Descriptions of two self-construals such as independent and interdependent selfconstruals can be utilised to help understand individuals' differences in terms of behavioural intentions.

Regulatory Focus Theory (Higgins, 1997, 1998) was also examined as the foundation for an individual variable in this study. Regulatory focus can be conceptualised as the message (e.g., gain/approach versus loss/avoidance) or viewers' regulatory focus (e.g., prevention-focused versus promotion-focused). The message's regulatory focus has received much attention, but the viewer's regulatory focus has been the subject of few studies; specifically, its potential role in the self-conscious emotional appeal effects is not clearly understood. More importantly, no studies have examined the effects of viewers' regulatory focus on the emotional message persuasiveness regarding its antecedent influential components, such as emotional arousal. This research utilised viewers' regulatory focus as prevention-focused versus promotion-focused in understanding its effect on emotional arousals elicited from guilt and shame appeals.

The current study investigated the role of personal cultural orientation in the effectiveness of guilt versus shame appeal messages in order to know in which individual culture guilt or shame is most effective. Most studies of guilt and shame have been proposed and tested across cultures based on ethnicity or nationality (e.g., Bagozzi et al., 2003; Lee \& Paek, 2014). This study used the personal cultural orientation (Yoo \& Donthu, 2005) to identify message receivers as individualists or collectivists at the individual level. It is argued that cultural differences are assumed to be consistent with individual differences within each of the cultures, these differences are not always reducible to individual differences (Na et al., 2010). Thus, this study furthers understanding of which personal cultural orientation people up-regulate or down-regulate these two guilt and shame selfconscious emotions.

Another significant contribution of this research lies in its focus on the health communications context and on the effectiveness of distinct types of emotions. As noted 
earlier, past studies on the relationship between guilt appeals and behavioural intentions have been focused on pro-social and charitable donation behaviour. There are differences among the pro-social or charitable donations and health communications contexts in terms of personal significance. This study differs from previous studies in that the health communications motivate the viewers for the benefit of themselves rather than for the benefit of others as in pro-social and charitable donation messages. The results add an additional aspect to the current body of knowledge by providing evidence that guilt and shame appeals operate differently due to the interaction between the self-conscious type of such emotions and the personal significance in the health communications context. The suggestion is that, even with the same type of emotions, their effectiveness apparently varies depending on the context. Such variations existing within self-conscious emotions appear to suggest that there may be the same or even more-marked differences for other emotions. This finding is important for theory building in discrete emotions and in other pro-social and pro-health contexts.

Finally, the findings of this study provide more empirical evidence of the effects of guilt versus shame appeals in the persuasive communications. A review of the psychology literature showed that most of the past studies on guilt versus shame found these two emotions have the differential effect in encouraging compliance. Specifically, guilt tends to create more favourable behavioural responses while shame is more likely to trigger negative behaviours (Abe, 2004; Lindsay-Hartz, 1984; Orth et al., 2010; Wong \& Tsai, 2007). However, this study did not find the differential main effect of the emotion type on message compliance, based on the total effects, including the direct effect from guilt/shame arousal to message compliance and the indirect effect via coping responses. This means that guilt arousal and shame arousal are equal in predicting message compliance. As previously discussed, in the present study, coping responses are the mechanism through which guilt and shame arousals lead to compliance with emotional appeal messages. Existence of the mediation effect of coping responses is one of the reasons explaining the varying findings. The study's finding suggests that individuals who feel guilt or shame emotions aroused from health communications are likely to cope adaptively to these emotions and eventually comply with the health message, regardless of emotion type. In addition, the effect of guilt versus shame arousals on message compliance varies across personal cultural orientations. Explicitly, guilt arousals predicted message compliance in all orientations, while shame arousals predicted 
message compliance only in collectivist, not in individualist, orientations. This study contributes to the extant literature as the first step of an empirical effort to probe the differential effect of guilt versus shame appeals under both impacts - mediating and moderating.

To conclude, the key contributions of the thesis are:

1. Development and testing of the fully extended model of the effectiveness of guilt versus shame appeals in health communications where a coping response is instrumental;

2. Provision of empirical support to the Cognitive-Motivational-Relational Theory of Emotion to understand self-conscious emotion-driven behaviours influenced by the coping response;

3. Identification of under-studied moderating factors (i.e., self-construal, regulatory focus, personal cultural orientation of individuals) and extension of the current research by examination of their moderation effects on the relationships of emotional appeals-emotional arousals-message compliance;

4. Isolation of the effects of unintentional emotions by examining guilt and shame separately through specific emotional arousals rather than emotional appeals;

5. Differentiation of the effect of guilt versus shame appeals in the context of health communications, specifically anti-binge drinking.

\subsection{Methodological Contributions}

\subsubsection{The Development of a New Set of Stimulus Materials}

Beyond theoretical significance, the development of a new set of stimulus materials in the study's experiments can make a methodological contribution. Specifically, the print ads were newly developed based on the notion that focuses on specific behaviour versus the self to manipulate guilt and shame arousals in subjects, respectively (Agrawal \& Duhachek, 2010; Boudewyns et al., 2013; Duhachek et al., 2012). Exceptionally, these stimuli in the present study differentiated guilt and shame by varying only in the body copy section as treatment conditions so as to provide a more carefully controlled comparison. This helps rectify the manipulation issue caused by employing different topics, different illustrations, and different shame- or guilt-eliciting message tactics for both within and between the shame and guilt appeals in many previous studies (see 
Boudewyns et al., 2013). Therefore, the new set of stimulus ads would help avoid the criticisms about the validity of theoretical settings as an experimental technique.

\subsubsection{Visual Capability}

It is that the possibility of inducing self-conscious emotional guilt or shame through the design of an ad; for instance, by the wording and/or the visual rather than the recall of an emotional episode. To date, many research studies have utilised the latter technique to elicit guilt and shame (e.g., Agrawal \& Duhachek, 2010; Han et al., 2014). For example, in Agrawal and Duhachek's (2010) studies on defensive processing of threatening health messages, the subjects were asked to recall an emotional event in which they experienced extreme guilt/shame, then they were instructed to write in detail their thoughts and feelings related to this event to prime incidental guilt/shame emotions. In this study, the stimulus ads incorporated characteristics of both content and design (Paul et al., 1997). The focal guilt and shame appeals were developed based on the literature of guilt and shame in binge drinking (Agrawal \& Duhachek, 2010; Duhachek et al., 2012). The male and female images modified from the two previous guilt and shame Agrawal and Duhachek's (2010) and Duhachek et al.'s (2012) studies in binge drinking and they were used such that male participants were exposed to a male image and females the female image. The study adopted the Adapted Shame and Guilt Scale (Hoblitzelle, 1987) to measure shame and guilt arousal. The scale is a generalised scale divorcing shame and guilt from the specific contexts in which they arise and make no reference to specific elements (e.g., alcohol use disorder) of either emotion. This generalised scale is consistent with the nature of guilt (e.g., specific behaviour) and shame (e.g., global self) in this study. By using a different method to induce guilt and shame emotions, the current study increases generalisability of the findings (Tiedens \& Linton, 2001).

\subsection{Practical Implications}

The findings of this study have important practical implications. The insights provided by this research could help health marketers, policy makers as well as health promotion agencies to effectively develop health communication campaigns with more appealing message content and appropriate media selection.

Firstly, guilt and shame as self-conscious emotions frequently involve perceptions of the self and are thus particularly persuasive tools for health communicators in addressing a 
wide range of unhealthy behaviours, such as binge drinking, drink driving, and smoking. Messages can focus on threats posed to personal notions of self-integrity highlighting guilt and shame, or on the transgression of a person with respect to others (i.e., selfreferenced versus other-referenced). The use of such message strategies will enhance the effectiveness of self-conscious emotional appeals in practice.

The higher emotional arousals can be characterised as instruments and are found to lead to more adaptive coping responses among message receivers. To create greater emotional arousals, practitioners should highlight attributes of guilt (i.e., specific behaviour) or shame (i.e., the self) in their promotion and communication strategies, combined with individual differences valued by the message receivers. In addition to arousing guilt and shame as they relate to harmful behaviours, health messages may trigger adaptive coping responses to these emotions and, together, provide the greatest health message compliance. Health marketers should include the aspect of coping responses as a tool for achieving greater effectiveness in their advertising and promotional elements in order to improve consumers' intentions to comply with their health communications plans.

The finding that coping responses in emotional arousals are indeed important indicates a prospect for health marketers and promotion agencies to create health communications and public announcements that result in stronger adaptive coping responses. Recent research indicated a mechanism that increases adaptive responses and makes receivers more accepting of health messages could be self-affirmation (see de Wit, Das, \& de Hoog, 2007). Self-affirmation is seen as the affirmation of values that are important to the self (Steele, 1988). The affirmation of an important value that is unrelated to health can function as a buffer against the self-regulatory costs of a threatening message and enhance adaptive responses to the health message. This is similar to the way in which psychological resources of optimism, personal control and meaning have been found to buffer people against psychological as well as physical adversity (Taylor \& Brown, 1988; Taylor, Kemeny, Reed, Bower, \& Gruenewald, 2000). Practically, to induce selfaffirmation, the message attempts to make receivers think about positive aspects of themselves, to focus their attention on who they are, to become aware of what they value about themselves, and to think about things personally important to them and about their values (e.g., Harris et al., 2007). 
Secondly, the research suggests that for guilt, the self-referenced appeals are more effective in arousing guilt in the message receivers rather than the other-referenced ones. Instead of focusing a message only on the behaviour to evoke a general guilt arousal that has no great effect on the anticipated binge drinker, the key is whether highlighting the message receiver's behaviour (e.g., YOU are a transgressor) rather than the others' behaviour (e.g., OTHERS are transgressors) will be more effective. Thus, health marketing messages are more effective if more emphasis is placed on self-reference of the message receivers. On the other hand, for shame, both self-referencing appeals are equally effective in arousing shame in the message receivers. The use of such message tactics will enhance the effectiveness of guilt and shame appeals in application.

Thirdly, the insights of self-construal, regulatory focus and personal cultural orientation differences in message receivers can provide communication campaigners with a means for targeting audiences at their tactical level. The most obvious consideration is the selection of a segmentation basis by using these individual differences. Market segmentation is seen as an important component of many successful marketing strategies because an understanding of segment characteristics is essential for marketing communications. Segmentation for such guilt and shame appeal communications can be achieved through studying the media or technology habits of message receivers. A judgement can be made regarding the collectivism level of the target consumer of a media vehicle or website where the message is being considered on the basis of preferred content and featured articles. For instance, heavy users of social networking sites such as Facebook who keep in contact with their family and close friends on the site are likely to be collectivists (Martin et al., 2013) and would suit shame appeal messages. Similarly, prevention-focused people suit shame appeal messages and independent people suit guilt appeal messages. Thus, when the audiences are segmented on the basis of self-construal, regulatory focus and personal cultural orientation, health communication campaigns that reflect, and are better suited to, the individualities of consumer behaviour may then be considered. While the message characteristics are under the communication campaigner's control, it is possible to target specific audiences through the choice of media programmes/channels as communication vehicles.

Lastly, the study found that types of emotion interacting with self-construal, regulatory focus and personal cultural orientation impact on guilt and shame appeal effectiveness. 
Specifically, independents have higher guilt arousals than interdependents, preventionfocused individuals have higher shame arousals than promotion-focused individuals, and shame arousals are stronger in predicting the message compliance for collectivists than for individualists. By exploring emotional appeals with different types of emotion, alongside their varying evaluations of certain message receiver attributes, health marketers could determine the appropriate message content and media channel for health communications and design an overall marketing campaign based on these elements. Consequently, health marketers, policy makers as well as health promotion agencies need to select the right media channels, giving them an appropriate message based on a distinct emotion (guilt versus shame). For example, the higher the independence of the target audience, the more likely the compliance with the guilt appeal message.

In all, consumers frequently experience guilt or shame in daily life, resulting from engaging in unhealthy behaviours such as binge drinking, drinking and driving or smoking. Since these two emotions are connected to many harmful behaviours, practitioners could use these two emotions in communications to enhance the message persuasion. Given their prevalence in consumers' experience and health communications, it is critical to understand how these discrete emotions affect how consumers cope with the message and make subsequent choices. This is especially important because most social marketers treat guilt and shame as interchangeable emotions, although this research theorises that each activates a distinct psychological mind-set. Armed with this understanding of how each emotion works, health marketers can better structure emotional messaging and select media that facilitate health-promoting consumer behaviour.

\subsection{Limitations and Future Research Directions}

\subsubsection{Limitations of the Study}

This study has some limitations that should be taken into account when the findings of this study are interpreted and applied. While this study can assist global health communicators, and anti-binge drinking in particular, to develop health communications campaigns, the study's results are limited to New Zealand. Despite a multi-cultural blend within its population, individualists dominate in this country (Scollon et al., 2011). It is argued that in the case of personal cultural orientation, a one-size-fits-all approach may 
not apply. Thus, further advances in this area require investigating samples in Eastern countries.

The findings of the current study are also limited to health communications. Given this, generalising the results to other contexts, such as a commercial one, must be made carefully. There is a possibility that the behavioural patterns of consumers in other contexts could vary from those exposed in this study. Therefore, generalisation of this study's findings is limited to pro-social behaviours in other social issues focusing more less on the benefit of others (e.g., donations, voting behaviour). In addition, the present study does not include all possible variables in the model of health communications as it is difficult to cover all these determinants in a single model. Further, the questionnaire using as a survey instrument in the study should be kept comparatively short to encourage participants to take part.

Information for independent and dependent variables was sourced from the same respondents that could generate the common method bias. Also, due to the sensitive nature of guilt and shame emotions their measurement items could produce social desirability biases (Cohen, Wolf, Panter, \& Insko, 2011). With regards to the concern about social desirability on sensitive topics, the study conducted Harman's single factor test and marker variable test to examine the common method variance (CMV). Based on the CMV test, although it can be argued that the findings are safe from common method bias, the results need to be interpreted with caution. It is challenging to question respondents about their self-conscious emotional feelings and responses. However, this study did not face any difficulties in this regard because of the use of self-administered online survey method and anonymity.

An additional limitation is found in the research method of study. Although self-report measures are recommended for assessing emotional states, they only provide information about conscious experiences of aroused feelings of guilt and shame. Next, as previously mentioned, type of guilt appeals (i.e., anticipatory) as controlled in this study, could be examined in future research. Moreover, a longitudinal study investigating the differential long-term effects of guilt and shame appeals would also be desirable. Further, the nature of an undergraduate student sample, even though relevant in this study, with limited age range and educational level could also restrict the study's generalisability. Finally, actual behaviours rather than behavioural intentions would also be desirable. 
It is expected that the main findings of this study will be significant in health communications, regardless of the limitations stated above. The present findings contribute to useful insights into this aspect of positive behaviour and the combination of other factors is left for future research.

\subsubsection{Directions for Future Research}

The role of self-conscious emotions such as guilt and shame in influencing certain aspects of coping responses and behavioural intentions, as well as the importance of increasing health marketers/researchers' understanding of the relationship between emotional arousals and coping responses, appears obvious. This study provides a foundation for further research on self-conscious emotions and persuasive health messages.

In this study, the interactive effect of individuals' self-construals with either selfreferencing or the sources of evaluation was found not to be significant. While independent individuals have sub-types (e.g., relational self-construal versus independent self-construal) that may vary effects on cognition, affect, and motivation (Cross et al., 2010), future research should perhaps investigate this aspect of sub-types and compare relational self-construal versus independent self-construal effects across self- and otherreference or internal and external sources of evaluation for guilt/shame arousals. A further study examining how independent self-construals interacting with self-referencing or the sources of evaluation affect self-emotional guilt/shame arousals would suggest possible implications for health communications planners.

Instead of moderation, it will be interesting to examine self-referencing as the mediator of emotional arousals. Self-referencing can favourably influence emotional responses when a receiver's self-construal and the specific emotion in a message are congruent. This suggestion is based on previous research (Martin et al., 2013; Martin, Lee, \& Yang, 2004; Meyers-Levy \& Peracchio, 1996). Thus, a self-referencing perspective represents a useful framework for future research in this area.

Self-construals were originally proposed to explain cross-cultural differences in cognition, emotion, and motivation (Markus \& Kitayama, 1991); however, a withinculture examination, conducted to allow for a direct examination of the causal role of self-construals, was a limitation for the current work. Based on the notion that positive distinctiveness and social connection are universal motives that differ in their chronic 
accessibility across cultures, future studies should prime self-construal that temporarily activates these motives to elicit the same psychological effects as does this study that measures self-construals. For example, asking participants to consider how they are different from (similar to) their family and friends, thus making an independent/interdependent self-construal temporarily accessible (Dean \& Fles, 2016; Trafimow, Triandis, \& Goto, 1991). Similarly, self-referencing can be primed, temporarily salient self-referenced versus other-referenced. It may be possible to induce self-referencing through the design of an ad; for instance, by wording or a visual. To date, no research studies have utilised priming techniques to directly examine the causal influence of self-referencing on guilt- and shame-related cognitions.

Likewise for research on binge drinking, future work should consider the role of a consumer's view of time and the future, such as their temporal orientation as presentoriented or future-oriented (Martin, Gnoth, \& Strong, 2009), and how this influences their evaluation of the health consequences of their actions (Martin et al., 2013).

The potential impact of a given message matched to a target market warrants careful selfreport assessment of dimensions of arousal response. Thus, a robust multidimensional 'picture of psychophysiological arousal response' can be evaluated without use of cumbersome, intrusive physiological measurement hardware, such as a galvanic skin response device (LaTour, Pitts, \& Snook-Luther, 1990; LaTour \& Rotfeld, 1997), or a heart rate monitor (Denson, Grisham, \& Moulds, 2011).

\subsection{Conclusion}

This research aimed to examine the mechanism through which guilt and shame appeals motivate health message compliance. Specifically, to examine the mediating roles of guilt and shame arousals and coping responses, and the moderating roles of self-construal (interacting with self-referencing and the sources of evaluation), regulatory focus, and personal cultural orientation. Below each of the research questions is sequentially returned to and discussed in light of the study's findings, and followed by a final overview of the study. 


\subsubsection{Research Question One: Do Self-Construal, Regulatory Focus and Personal Cultural Orientation Influence the Relative Effectiveness of Guilt and Shame Appeals towards Health Message Compliance?}

The findings provide support for the moderating effects of self-construal on guilt/shame arousals. Specifically, although the interactive effects with self-referencing or the sources of evaluation on guilt/shame arousals were not found in the present study, the main effect of self-construal on guilt arousals was evident. As an individual characteristic, selfconstrual moderates the levels of guilt arousals from guilt appeals. Explicitly, independent self-construals experience stronger guilt arousals than their interdependent counterparts when exposed to guilt appeals.

The findings also provide support for the moderating effects of regulatory focus on guilt/shame arousals. Specifically, regulatory focus moderates the levels of shame arousals from shame appeals. That is, prevention-focused people experience stronger shame arousals than their promotion-focused counterparts when exposed to shame appeals.

The above findings provide an insight when comparing the moderating effects of selfconstrual and regulatory focus between guilt versus shame emotional types and contrasting these findings with the moderation effects towards self-conscious emotional arousals in general. That is, although guilt and shame are self-conscious emotions, when comparing both in terms of distinct emotion, not all self-conscious emotions are equally appraised by individuals (Han et al., 2014).

In addition, the results indicate that personal cultural orientation has the moderating effect on message compliance across guilt and shame. The impact of shame arousals on message compliance is moderated by personal cultural orientation. However, the shame does not apply for guilt arousals. More specifically, shame arousals predict message compliance in only collectivist, not individualist, orientations, but guilt arousals predict message compliance in both orientations. Further, there is no difference in message compliance between individualists and collectivists, regardless of guilt or shame. However, there is a difference in the predictive power of guilt versus shame arousals on message compliance across individualists and collectivists. This is consistent with previous predictions that guilt is more favourable for behavioural responses than shame (Abe, 2004; Ghorbani et al., 2013). For individualists, when deciding on the level of 
compliance with the health message, shame is not a determinant of the amount, whereas guilt is a significant predictor of message compliance.

It is worth noting that, at the national level, the findings are consistent with previous cross-culture studies. In respect to shame, some authors argue that coping with the emotion depends on the cultural concept of self, which is different across cultures (Bagozzi et al., 2003; Dean \& Fles, 2016; Fontaine et al., 2002; Kitayama et al., 1995; Mesquita \& Karasawa, 2004; Wallbott \& Scherer, 1995). These authors suggest that in the collectivist cultures, showing shame to others is seen as courageous and positive, because it is an essential part of social interaction. This difference is probably due to differences in coping responses with shame. People in collectivist cultures seem to be more able to cope with shame adaptively. Collectivist people see their sense of self depending on social relationships, and the only possibility for recovering the positive sense of self is to repair their relationships. By contrast, people in individualist cultures are more likely to react in maladaptive ways which harm their interpersonal relationships. This is possibly because an easier way to relieve shame for individualist people is to avoid others or shift the blame to someone else rather than take responsibility for the matter (Silfver, 2007; Sznycer et al., 2016).

In overview, self-construal, regulatory focus and personal cultural orientation influence the arousals of guilt and shame appeals. The current study contributes to the extant literature as the first step of an empirical effort to differentiate the effect of guilt versus shame under influential individual differences. Therefore, the findings provide insight into when and why guilt and shame have differential effects on message processing and compliance.

\subsubsection{Research Question Two: Does the Coping Response to Guilt and Shame Arousals Influence Health Message Compliance?}

The results of this study indicate that coping response has a partial effect on the relationship of guilt/shame arousals and message compliance. This means that compliance with the health message is influenced by not only the coping response but also guilt/shame arousals. The existence of the mediation effect of coping responses is important in clarifying the inconsistency in the effect of guilt and shame arousals. Previous studies on pro-social and donation behaviours suggest that guilt and shame are distinct in terms of behavioural intentions (Orth et al., 2010; Wong \& Tsai, 2007). These 
studies supported guilt with more subsequent approach behaviours than shame. However, the current study on health communications did not find the differential effect of the distinct emotion type on message compliance [based on the total effects of the direct effect from guilt/shame arousals, and the indirect effect via coping responses, to message compliance]. In this thesis, coping response is found to be the mechanism through which guilt and shame arousals lead to compliance with the health message. This suggests that people who feel the self-conscious emotions are likely to cope adaptively to these emotions and eventually comply with the health message, regardless of guilt or shame.

In this study, the health communications differs from pro-social and charitable donation in which the former motivates the viewers for the benefit of themselves while the latter stimulates for the benefit of others. This means that personal significance in this health communications context is significant. In addition, the presence of the mediation effect of coping responses is one of the reasons explaining the inconsistency in the differential effect of guilt and shame. The findings provided no evidence for an inverted U-shaped relationship in guilt (and shame) intensity and behavioural intentions. This inconsistency could be also explained with consideration of coping response. As supported by openended question coping responses, individuals who experience guilt or shame due to their integrity of self do not engage in a maladaptive coping response even when the emotion is strong (see more in Section 6.2.2). From the findings, it is clear that intense selfconscious emotional arousals are more likely to stimulate an adaptive coping response and, ultimately, compliance with the health message.

Therefore, the present study's findings not only support the notion that guilt tends to create more positive behaviours (Graton et al., 2016; Saintives \& Lunardo, 2016; Tangney et al., 2007), but also critique both the rationale that shame is innately maladaptive (Abe, 2004; Lindsay-Hartz, 1984; Stuewig et al., 2010) and the inverted Ushaped relationship (Coulter et al., 1999; Coulter \& Pinto, 1995; Ghingold \& Bozinoff, 1982; O'Keefe, 2002; Pinto \& Priest, 1991; Pinto \& Worobetz, 1992).

In overview, the thesis emphasises the importance of the coping response as a key mediator in motivating people to follow positive behaviours advocated in the health messages using guilt and shame. Accordingly, health communicators must not rely only on guilt or shame arousals as a mechanism but also on adaptive coping responses to secure message compliance in individuals. Communicators should incorporate guilt or 
shame arousals with adaptive coping responses into the health message. The two strategies working in tandem will provide the greatest result in terms of a favourable attitude towards the message and behaviours and, ultimately, positive behaviour change.

\subsubsection{Final Remarks}

Drawing upon literature of cognition, emotion, motivation and research focusing on guilt and/or shame appeals in pro-social and charitable donation behaviour, the present study has extended the understanding of the processes by which guilt and shame appeals lead to better message compliance, within the context of health communications. This study is the first to empirically assess the mediating effect of coping responses on the relationship of guilt/shame emotional arousal and message compliance from both categorised and continuous measurement perspectives. The assessment of coping responses in emotions has generated insightful findings into the significance of self-conscious emotions like guilt and shame and its implications for health communications knowledge and practice, particularly for preventive health advocacies such as anti-binge drinking.

The present study has specifically focused on health messages using guilt and shame appeals to examine the mediating roles of coping responses, and the moderating roles of regulatory focus, personal cultural orientation, and self-construal interacting with selfreferencing and the sources of evaluation. Findings show that, although the interactive effects of self-construal with self-referencing or the sources of evaluation on emotional arousals were nonsignificant, the main effects of self-construal and self-referencing on guilt arousal were significant. Consistently, regulatory focus and personal cultural orientation moderate the relationship of emotional appeal and emotional arousal and emotional arousal and message compliance, respectively. Most importantly, coping responses were consistently generated from emotional arousals and positively affected message compliance through the indirect route. The stronger the arousal generated in subjects, the greater their coping responses (adaptive) and, ultimately, behavioural intentions (in terms of message compliance).

The study's extended model demonstrates that the main flaw in traditional stimulusresponse appeal research is not ill-conceived theory, but rigid retention of assumptions and errors in the research process. The aim of investigating communications' effectiveness is not to determine an optimal level of emotion but rather to find the optimal type of emotion that a target segment will act on. In fact, the past studies have often 
examined the effects of guilt or shame appeals in persuasive messages. In traditional stimulus-response research, manipulation of guilt or shame was used to assess which threatening emotion produced the greatest response, and the role of the receiver's response was overlooked. That approach limited the practical generalisations beyond the stimulus materials and receivers of each particular study. This study's model, in contrast, allows inherent generalisations and applications beyond the specific research materials.

As the conceptual and empirical demonstration has shown, the pragmatic value of this research is in the study of emotional arousal/response from the stimulus rather the stimulus/appeal itself. With a standardised and developed model such as the one this study proposes, health marketers could determine individual messages and the most persuasive health message would be the one that stimulates the greatest emotional arousal with a given target audience segment. Given that different segments cope differently, with a detailed analysis of psychological coping responses, the model affords a basis for understanding the role of self-conscious emotional arousals in health communications and persuasiveness. By understanding that, more extensive generalisations, pragmatic applications, and extensions could be conducted in future research.

In summary, the current study provided theoretical clarification of literal guilt/shame appeal and its arousal, as well as a test of an extended model of coping response effects that can mediate in guilt and shame arousals to health message compliance. Logically, future research should expand the model and examine receivers' responses in other contexts with different messages. But most importantly, the model primarily provides an anchor for consistency and extension of persuasive messages using self-conscious emotions. 


\section{References}

Aaker, D., Kumar, V., Day, G., Lawley, M., \& Stewart, D. (2007). Marketing Research (2nd ed.). Milton, Queensland: John Wiley \& Sons.

Aaker, J. L. (2000). Accessibility or Diagnosticity? Disentangling the Influence of Culture on Persuasion Processes and Attitudes. Journal of Consumer Research, 26(4), 340-357.

Aaker, J. L., \& Lee, A. Y. (2001). "I" Seek Pleasures and "We" Avoid Pains: The Role of Self-Regulatory Goals in Information Processing and Persuasion. Journal of Consumer Research, 28(1), 33-49.

Aaker, J. L., \& Williams, P. (1998). Empathy versus Pride: The Influence of Emotional Appeals across Cultures. Journal of Consumer Research, 25(3), 241-261.

Abe, J. A. (2004). Shame, Guilt, and Personality Judgment. Journal of Research in Personality, 38(2), 85-104.

Acock, A. C., \& Martin, J. D. (1974). The Undermeasurement Controversy: Should Ordinal Data Be Treated as Interval? Sociology and Social Research, 58(4), 427433.

Agrawal, N., \& Duhachek, A. (2010). Emotional Compatibility and the Effectiveness of Antidrinking Messages: A Defensive Processing Perspective on Shame and Guilt. Journal of Marketing Research, 47(2), 263-273.

Agrawal, N., Menon, G., \& Aaker, J. L. (2007). Getting Emotional about Health. Journal of Marketing Research, 44(1), 100-113.

Alden, D. L., \& Crowley, A. E. (1995). Sex Guilt and Receptivity to Condom Advertising. Journal of Applied Social Psychology, 25(16), 1446-1463.

Alegria, M., Atkins, M., Farmer, E., Slaton, E., \& Stelk, W. (2010). One Size Does Not Fit All: Taking Diversity, Culture and Context Seriously. Administration and Policy in Mental Health and Mental Health Services Research, 37(1-2), 48-60.

Allen, P. J., \& Bennett, K. (2010). PASW Statistics by SPSS: A Practical Guide: Version 18.0. Melbourne, Victoria: Cengage Learning Australia.

Anderson, J. C., \& Gerbing, D. W. (1988). Structural Equation Modeling in Practice: A Review and Recommended Two-Step Approach. Psychological Bulletin, 103(3), 411-423. 
Andrews, J. C., Durvasula, S., \& Akhter, S. H. (1990). A Framework for Conceptualizing and Measuring the Involvement Construct in Advertising Research. Journal of Advertising, 19(4), 27-40.

Ang, S. H., \& Low, S. Y. (2000). Exploring the Dimensions of Ad Creativity. Psychology and Marketing, 17(10), 835-854.

Arbuckle, J. (2013). Amos 22 User's Guide. Crawfordville, FL: Amos Development Corporation.

Armitage, C. J., Norman, P., \& Conner, M. (2002). Can the Theory of Planned Behaviour Mediate the Effects of Age, Gender and Multidimensional Health Locus of Control? British Journal of Health Psychology, 7(3), 299-316.

Arthur, D., \& Quester, P. (2004). Who's Afraid of that Ad? Applying Segmentation to the Protection Motivation Model. Psychology and Marketing, 21(9), 671-696.

Averill, P. M., Diefenbach, G. J., Stanley, M. A., Breckenridge, J. K., \& Lusby, B. (2002). Assessment of Shame and Guilt in a Psychiatric Sample: A Comparison of Two Measures. Personality and Individual Differences, 32(8), 1365-1376.

Bagozzi, R. P., \& Baumgartner, H. (1994). The Evaluation of Structural Equation Models and Hypothesis Testing. In R. P. Bagozzi (Ed.), Principles of Marketing Research (pp. 386-422). Cambridge, MA: Blackwell.

Bagozzi, R. P., \& Yi, Y. (1988). On the Evaluation of Structural Equation Models. Journal of the Academy of Marketing Science, 16(1), 74-94.

Bagozzi, R. P., \& Yi, Y. (2012). Specification, Evaluation, and Interpretation of Structural Equation Models. Journal of the Academy of Marketing Science, 40(1), 8-34.

Bagozzi, R. P., Verbeke, W., \& Gavino, J. C. (2003). Culture Moderates the SelfRegulation of Shame and Its Effects on Performance: The Case of Salespersons in the Netherlands and the Philippines. Journal of Applied Psychology, 88(2), 219233.

Bagozzi, R. P., Yi, Y., \& Phillips, L. W. (1991). Assessing Construct Validity in Organizational Research. Administrative Science Quarterly, 36(3), 421-458.

Baron, R. M., \& Kenny, D. A. (1986). The Moderator-Mediator Variable Distinction in Social Psychological Research: Conceptual, Strategic, and Statistical Considerations. Journal of Personality and Social Psychology, 51(6), 1173-1182.

Basil, D. Z., Ridgway, N. M., \& Basil, M. D. (2001). Guilt Appeals: The Effects of Responsibility and Altruistic Norms. Advances in Consumer Research, 28, 216. 
Basil, D. Z., Ridgway, N. M., \& Basil, M. D. (2006). Guilt Appeals: The Mediating Effect of Responsibility. Psychology and Marketing, 23(12), 1035-1054.

Basil, D. Z., Ridgway, N. M., \& Basil, M. D. (2008). Guilt and Giving: A Process Model of Empathy and Efficacy. Psychology and Marketing, 25(1), 1-23.

Baumeister, R. F., Stillwell, A. M., \& Heatherton, T. F. (1994). Guilt: An Interpersonal Approach. Psychological Bulletin, 115(2), 243-267.

Baumeister, R. F., Stillwell, A. M., \& Heatherton, T. F. (1995). Personal Narratives about Guilt: Role in Action Control and Interpersonal Relationships. Basic and Applied Social Psychology, 17(1-2), 173-198.

Baumgartner, H., \& Homburg, C. (1996). Applications of Structural Equation Modeling in Marketing and Consumer Research: A Review. International Journal of Research in Marketing, 13(2), 139-161.

Bécheur, I., Dib, H., Merunka, D., \& Valette-Florence, P. (2007). Emotions of Fear, Guilt or Shame in Anti-Alcohol Messages: Measuring Direct Effects on Persuasion and the Moderating Role of Sensation Seeking. Paper presented at the European Conference of the Association for Consumer Research, Milan, Italy.

Belch, G. E., Belch, M. A., \& Jones, M. A. (1995). An Exploratory Investigation of Teenagers' Attitudes toward Anti-Drug Appeals. European Advances in Consumer Research, 2, 329-336.

Benedict, R. (1946). The Chrysanthemum and the Sword: Patterns of Japanese Culture. Boston, NY: Houghton Mifflin.

Bennett, D. S., Sullivan, M. W., \& Lewis, M. (2005). Young Children's Adjustment as a Function of Maltreatment, Shame, and Anger. Child Maltreatment, 10(4), 311323.

Bennett, R. (1996). Effects of Horrific Fear Appeals on Public Attitudes towards AIDS. International Journal of Advertising, 15(3), 183-202.

Bennett, R. (1998). Shame, Guilt and Responses to Non-Profit and Public Sector Ads. International Journal of Advertising, 17, 483-500.

Bentler, P., \& Stein, J. (1992). Structural Equation Models in Medical Research. Statistical Methods in Medical Research, 1(2), 159-181.

Block, L. G. (2005). Self-Referenced Fear and Guilt Appeals: The Moderating Role of Self-Construal. Journal of Applied Social Psychology, 35(11), 2290-2309.

Blum, A. (2008). Shame and Guilt, Misconceptions and Controversies: A Critical Review of the Literature. Traumatology, 14(3), 91-102. 
Bollen, K. A. (1984). Multiple Indicators: Internal Consistency or No Necessary Relationship? Quality and Quantity, 18(4), 377-385.

Boudewyns, V., Turner, M. M., \& Paquin, R. S. (2013). Shame-Free Guilt Appeals: Testing the Emotional and Cognitive Effects of Shame and Guilt Appeals. Psychology and Marketing, 30(9), 811-825.

Boulos, M. N. K., \& Wheeler, S. (2007). The Emerging Web 2.0 Social Software: An Enabling Suite of Sociable Technologies in Health and Health Care Education. Health Information and Libraries Journal, 24(1), 2-23.

Brehm, J. W. (1966). A Theory of Psychological Reactance. New York: Academic Press.

Brennan, L., \& Binney, W. (2010). Fear, Guilt, and Shame Appeals in Social Marketing. Journal of Business Research, 63(2), 140-146.

Brommersma, J. (2007). A Charity's Marathon: Charity Advertising, The Telethon and Consumer Response. University of Nottingham.

Brown, P., Keenan, J. M., \& Potts, G. R. (1986). The Self-Reference Effect with Imagery Encoding. Journal of Personality and Social Psychology, 51(5), 897-906.

Bull, F. C., Holt, C., Kreuter, M., Clark, E., \& Scharff, D. (2001). Understanding the Effects of Printed Health Education Materials: Which Features Lead to Which Outcomes? Journal of Health Communication, 6(3), 265-280.

Burgoon, M., Alvaro, E., Grandpre, J., \& Voulodakis, M. (2002). Revisiting the Theory of Psychological Reactance. In J. P. Dillard \& M. Pfau (Eds.), The Persuasion Handbook: Developments in Theory and Practice (pp. 213-232). Thousand Oaks, CA: Sage Publications.

Burnett, M. S., \& Lunsford, D. A. (1994). Conceptualizing Guilt in the Consumer Decision-Making Process. Journal of Consumer Marketing, 11(3), 33-43.

Burnkrant, R. E., \& Unnava, H. R. (1995). Effects of Self-Referencing on Persuasion. Journal of Consumer Research, 22, 17-26.

Burns, R. P., \& Burns, R. (2008). Business Research Methods and Statistics Using SPSS. Los Angeles: Sage Publications.

Byrne, B. M. (2010). Structural Equation Modeling with AMOS: Basic Concepts, Applications, and Programming (2nd ed.). New York: Routledge.

Carey, R. N., McDermott, D. T., \& Sarma, K. M. (2013). The Impact of Threat Appeals on Fear Arousal and Driver Behavior: A Meta-Analysis of Experimental Research 1990-2011. PLoS One, 8(5), 1-8. 
Carey, R. N., \& Sarma, K. M. (2011). The Impact of Threat Appeal Messages on Risky Driving Intentions: A Terror Management Theory perspective. Journal of the Australasian College of Road Safety, 22(4), 51-56.

Cashell-Smith, M. L., Connor, J. L., \& Kypri, K. (2007). Harmful Effects of Alcohol on Sexual Behaviour in a New Zealand University Community. Drug and Alcohol Review, 26(6), 645-651.

Cavana, R. Y., Delahaye, B. L., \& Sekaran, U. (2001). Applied Business Research: Qualitative and Quantitative Methods. Milton, Queensland: John Wiley \& Sons.

Cecilia, A. E., \& Delyse, H. (2008). Alcohol Use, Abuse and Dependence. In A. E. Cecilia (Ed.), Adolescent Addiction: Epidemiology, Assessment, and Treatment. San Diego, CA: Academic Press.

Chang, S.-J., Van Witteloostuijn, A., \& Eden, L. (2010). From the Editors: Common Method Variance in International Business Research. Journal of International Business Studies, 41(2), 178-184.

Cheung, R. Y. M., \& Park, I. J. K. (2010). Anger Suppression, Interdependent SelfConstrual, and Depression among Asian American and European American College Students. Cultural Diversity and Ethnic Minority Psychology, 16(4), 517525 .

Chin, W. W. (1998). Commentary: Issues and Opinion on Structural Equation Modeling. MIS Quarterly, 22(1), vii-xvi.

Cho, H. (2000). Public Opinion as Personal Cultivation: A Normative Notion and a Source of Social Control in Traditional China. International Journal of Public Opinion Research, 12(3), 299-323.

Cleary, M. J. (1992). Shame and Shame-Based Syndromes: Implications for Health Education. Health Values: The Journal of Health Behavior, Education and Promotion, 16(6), 47-54.

Cohen, J. (1988). Statistical Power Analysis for the Behavioural Sciences. Hillside, NJ: Lawrence Earlbaum Associates.

Cohen, T. R., Wolf, S. T., Panter, A. T., \& Insko, C. A. (2011). Introducing the GASP Scale: a New Measure of Guilt and Shame Proneness. Journal of Personality and Social Psychology, 100(5), 947-966.

Comşa, M. (2010). How to Compare Means of Latent Variables across Countries and Waves: Testing for Invariance Measurement. An Application Using Eastern European Societies. Sociológia, 42(6), 639-669. 
Conner, K. R., Langley, J., Tomaszewski, K. J., \& Conwell, Y. (2003). Injury Hospitalization and Risks for Subsequent Self-Injury and Suicide: A National Study from New Zealand. American Journal of Public Health, 93(7), 1128-1131.

Cool, K., Dierickx, I., \& Jemison, D. (1989). Business Strategy, Market Structure and Risk-Return Relationships: A Structural Approach. Strategic Management Journal, 10(6), 507-522.

Cotte, J., Coulter, R. A., \& Moore, M. (2005). Enhancing or Disrupting Guilt: The Role of Ad Credibility and Perceived Manipulative Intent. Journal of Business Research, 58(3), 361-368.

Coulter, R. H., Cotte, J., \& Moore, M. (1997). Guilt Appeals in Advertising: Are You Feeling Guilty? Paper presented at the AAA Winter Marketing Educators, Chicago.

Coulter, R. H., Cotte, J., \& Moore, M. L. (1999). Believe It or Not: Persuasion, Manipulation and Credibility of Guilt Appeals. Advances in Consumer Research, 26(1), 288-294.

Coulter, R. H., \& Pinto, M. B. (1995). Guilt Appeals in Advertising: What Are Their Effects? Journal of Applied Psychology, 80(6), 697-705.

Creighton, M. R. (1990). Revisiting Shame and Guilt Cultures: A Forty-Year Pilgrimage. Ethos, 18(3), 279-307.

Creswell, J. W. (2009). Research Design: Qualitative, Quantitative, and Mixed Methods Approaches. London, England: Sage Publications.

Cropanzano, R., Paddock, L., Rupp, D. E., Bagger, J., \& Baldwin, A. (2008). How Regulatory Focus Impacts the Process-by-Outcome Interaction for Perceived Fairness and Emotions. Organizational Behavior and Human Decision Processes, 105(1), 36-51.

Cross, S. E., Hardin, E. E., \& Gercek-Swing, B. (2010). The What, How, Why, and Where of Self-Construal. Personality and Social Psychology Review, XX(X), 138.

Cross, S. E., Morris, M. L., \& Gore, J. S. (2002). Thinking about Oneself and Others: The Relational-Interdependent Self-Construal and Social Cognition. Journal of Personality and Social Psychology, 82(3), 399.

Crowe, E., \& Higgins, E. T. (1997). Regulatory Focus and Strategic Inclinations: Promotion and Prevention in Decision-Making. Organizational Behavior and Human Decision Processes, 69(2), 117-132. 
Curran, P. J., West, S. G., \& Finch, J. F. (1996). The Robustness of Test Statistics to Nonnormality and Specification Error in Confirmatory Factor Analysis. Psychological Methods, 1(1), 16-29.

Czub, T. (2013). Shame as a Self-Conscious Emotion and Its Role in Identity Formation. Polish Psychological Bulletin, 44(3), 245-253.

Datu, J. A. D. (2015). Validating the Revised Self-Construal Scale in the Philippines. Current Psychology, 34(4), 626-633.

Davidov, E., \& Depner, F. (2011). Testing for Measurement Equivalence of Human Values across Online and Paper-and-Pencil Surveys. Quality and Quantity, 45(2), 375-390.

Davis, J. J. (1997). Advertising Research: Theory and Practice. Upper Saddle River, NJ: Prentice Hall.

Dawson, D. A., Grant, B. F., Stinson, F. S., \& Chou, P. S. (2004). Another Look at Heavy Episodic Drinking and Alcohol Use Disorders among College and Noncollege Youth. Journal of Studies on Alcohol and Drugs, 65(4), 477-488.

de Hoog, N., Stroebe, W., \& de Wit, J. B. F. (2005). The Impact of Fear Appeals on Processing and Acceptance of Action Recommendations. Personality and Social Psychology Bulletin, 31(1), 24-33.

de Hooge, I. E., Breugelmans, S. M., \& Zeelenberg, M. (2008). Not So Ugly After All: When Shame Acts as a Commitment Device. Journal of Personality and Social Psychology, 95(4), 933-943.

de Hooge, I. E., Zeelenberg, M., \& Breugelmans, S. M. (2010). Restore and Protect Motivations following Shame. Cognition and Emotion, 24(1), 111-127.

De Pelsmacker, P., Cauberghe, V., \& Dens, N. (2011). Fear Appeal Effectiveness for Familiar and Unfamiliar Issues. Journal of Social Marketing, 1(3), 171-191.

de Wit, J. B., Das, E., \& de Hoog, N. (2007). Self-Regulation of Health Communications: A Motivated Processing Approach to Risk Perception and Persuasion. In M. Hewstone, H. A. W. Schut, J. B. F. de Wit, K. van den Bos, \& M. S. Stroebe (Eds.), The Scope of Social Psychology: Theory and Applications. New York: Psychology Press.

Dean, K. K., \& Fles, E. H. (2016). The Effects of Independent and Interdependent SelfConstruals on Reactions to Transgressions: Distinguishing Between Guilt and Shame. Self and Identity, 15(1), 90-106. 
Dearing, R. L., Stuewig, J., \& Tangney, J. P. (2005). On the Importance of Distinguishing Shame from Guilt: Relations to Problematic Alcohol and Drug Use. Addictive Behaviors, 30(7), 1392-1404.

Denson, T. F., Grisham, J. R., \& Moulds, M. L. (2011). Cognitive Reappraisal Increases Heart Rate Variability in Response to an Anger Provocation. Motivation and Emotion, 35(1), 14-22.

DeVellis, R. F. (2016). Scale Development: Theory and Applications (4th ed.). Thousand Oaks, CA: Sage publications.

Dickinson, S., \& Holmes, M. (2008). Understanding the Emotional and Coping Responses of Adolescent Individuals Exposed to Threat Appeals. International Journal of Advertising: The Quarterly Review of Marketing Communications, 27(2), 251-278.

Dickinson, S. J., \& Holmes, M. D. (2011). Threat Appeal Communications: The Interplay between Health Resistance and Cognitive Appraisal Processes. Journal of Marketing Communications, 17(2), 107-125.

Dillard, J. P., \& Nabi, R. L. (2006). The Persuasive Influence of Emotion in Cancer Prevention and Detection Messages. Journal of Communication, 56(Supplement s1), S123-S139.

Dillard, J. P., \& Peck, E. (2000). Affect and Persuasion Emotional Responses to Public Service Announcements. Communication Research, 27(4), 461-495.

Duhachek, A., Agrawal, N., \& Han, D. (2012). Guilt versus Shame: Coping, Fluency, and Framing in the Effectiveness of Responsible Drinking Messages. Journal of Marketing Research, 49(6), 928-941.

Efron, B., \& Tibshirani, R. J. (1994). An Introduction to the Bootstrap. New York: Chapman \& Hall.

Eid, M., \& Diener, E. (2001). Norms for Experiencing Emotions in Different Cultures: Inter- And Intranational Differences. Journal of Personality and Social Psychology, 81(5), 869-885.

Ekman, P., \& Friesen, W. V. (1975). Unmasking the Face: A Guide to Recognizing Emotions from Facial Cues. Englewood Cliffs, NJ: Prentice Hall.

Ellsworth, P. C. (1994). Some Reasons to Expect Universal Antecedents of Emotion. In P. Ekman \& R. J. Davidson (Eds.), The Nature of Emotion: Fundamental Questions (pp. 150-154). New York: Oxford University Press. 
Else-Quest, N. M., Higgins, A., Allison, C., \& Morton, L. C. (2012). Gender Differences in Self-Conscious Emotional Experience: A Meta-Analysis. Psychological Bulletin, 138(5), 947-981.

Englis, B. G. (1990). Consumer Emotional Reactions to Television Advertising and Their Effects on Message Recall. In S. J. Agres, J. A. Edell, \& T. M. Dbitsky (Eds.), Emotion in Advertising: Theoretical and Practical Applications (pp. 231-253). New York: Quorom Books.

Evans, J. R., \& Mathur, A. (2005). The Value of Online Surveys. Internet Research, 15(2), 195-219.

Evans, W. D., \& McCormack, L. (2008). Applying Social Marketing in Health Care: Communicating Evidence to Change Consumer Behavior. Medical Decision Making, 28(5), 781-792.

Feiring, C., \& Taska, L. S. (2005). The Persistence of Shame following Sexual Abuse: A Longitudinal Look at Risk and Recovery. Child Maltreatment, 10(4), 337-349.

Ferguson, T. J., Stegge, H., Miller, E. R., \& Olsen, M. E. (1999). Guilt, Shame, and Symptoms in Children. Developmental Psychology, 35(2), 347-357.

Fessler, D. M. (2001). Emotions and Cost-Benefit Assessment: The Role of Shame and Self-Esteem in Risk Taking. In R. Selten \& G. Gigerenzer (Eds.), Bounded Rationality: The Adaptive Toolbox (pp. 191-214). Cambridge, MA: MIT University Press.

Field, A. (2013). Discovering Statistics Using IBM SPSS Statistics (4th ed.). Thousand Oaks, CA: Sage Publications.

Flora, J. A., \& Maibach, E. W. (1990). Cognitive Responses to AIDS Information: The Effects of Issue Involvement and Message Appeal. Communication Research, 17(6), 759-774.

Folkman, S., Schaefer, C., \& Lazarus, R. S. (1979). Cognitive Processes as Mediators of Stress and Coping. In V. Hamilton \& D. M. Warburton (Eds.), Human Stress and Cognition: An Information Processing Approach (pp. 265-298). London, England: Wiley.

Fontaine, J. R., Poortinga, Y. H., Setiadi, B., \& Markam, S. S. (2002). Cognitive Structure of Emotion Terms in Indonesia and the Netherlands. Cognition and Emotion, 16(1), 61-86. 
Fornell, C., \& Larcker, D. F. (1981). Structural Equation Models with Unobservable Variables and Measurement Error: Algebra and Statistics. Journal of Marketing Research, 382-388.

Freimuth, V., Linnan, H. W., \& Potter, P. (2000). Communicating the Threat of Emerging Infections to the Public. Emerging Infectious Diseases, 6(4), 337-347.

Friestad, M., \& Wright, P. (1994). The Persuasion Knowledge Model: How People Cope with Persuasion Attempts. Journal of Consumer Research, 21, 1-31.

Frijda, N. H., Kuipers, P., \& Ter Schure, E. (1989). Relations among Emotion, Appraisal, and Emotional Action Readiness. Journal of Personality and Social Psychology, $57(2), 212-228$.

Fritz, M. S., \& MacKinnon, D. P. (2007). Required Sample Size to Detect the Mediated Effect. Psychological Science, 18(3), 233-239.

Galesic, M. (2006). Dropouts on the Web: Effects of Interest and Burden Experienced during an Online Survey. Journal of Official Statistics, 22(2), 313-328.

Gao, J., Wang, A., \& Qian, M. (2010). Differentiating Shame and Guilt from a Relational Perspective: A Cross-Cultural Study. Social Behavior and Personality, 38(10), 1401-1407.

Garson, G. D. (2012). Testing Statistical Assumptions. Asheboro, NC: Statistical Publishing Associates.

Garton, L., Haythornthwaite, C., \& Wellman, B. (1999). Studying On-Line Social Networks. In S. Jones (Ed.), Doing Internet Research: Critical Issues and Methods for Examining the Net. Thousand Oaks, CA: Sage Publications.

Gaskin, J. (2012a). General Guidelines. Gaskination's StatWiki. Retrieved from http://statwiki.kolobkreations.com/index.php?title=Guidelines\#Example_Analysis

Gaskin, J. (2012b). Structural Equation Modeling. Gaskination's StatWiki. Retrieved from http://statwiki.kolobkreations.com/index.php?title=Structural_Equation_Modeling\# Controls

Gausel, N., \& Brown, R. (2012). Shame and Guilt-Do They Really Differ in Their Focus of Evaluation? Wanting to Change the Self and Behavior in Response to Ingroup Immorality. The Journal of Social Psychology, 152(5), 547-567.

Gausel, N., \& Leach, C. W. (2011). Concern for Self-Image and Social Image in the Management of Moral Failure: Rethinking Shame. European Journal of Social Psychology, 41(4), 468-478. 
Ghingold, M. (1980). Guilt Arousing Marketing Communications: An Unexplored Variable Advances in Consumer Research (pp. 442-448). Michigan: Ann Arbor.

Ghingold, M., \& Bozinoff, L. (1982). Construct Validation and Empirical Testing of Guilt Arousing Marketing Communications. In A. Mitchell (Ed.), Advances in Consumer Research (Vol. 9, pp. 210-214). Arbor, MI: Association for Consumer Research.

Ghorbani, M., Liao, Y., Çayköylü, S., \& Chand, M. (2013). Guilt, Shame, and Reparative Behavior: The Effect of Psychological Proximity. Journal of Business Ethics, 114(2), 311-323.

Giguère, A., Légaré, F., Grimshaw, J., Turcotte, S., Fiander, M., Grudniewicz, A., . . . Gagnon, M. P. (2012). Printed Educational Materials: Effects on Professional Practice and Healthcare Outcomes. Cochrane Database of Systematic Reviews, (10), 1-197.

Gilbert, P. (1998). What Is Shame? Some Core Issues and Controversies. In P. E. Gilbert \& B. E. Andrews (Eds.), Shame: Interpersonal Behavior, Psychopathology, and Culture. New York: Oxford University Press.

Gilbert, P. (2000). The Relationship of Shame, Social Anxiety and Depression: The Role of the Evaluation of Social Rank. Clinical Psychology and Psychotherapy, 7(3), 174-189.

Gilbert, P., Allan, S., \& Goss, K. (1996). Parental Representations, Shame, Interpersonal Problems, and Vulnerability to Psychopathology. Clinical Psychology and Psychotherapy, 3(1), 23-34.

Gilbert, P., Bhundia, R., Mitra, R., McEwan, K., Irons, C., \& Sanghera, J. (2007). Cultural Differences in Shame-Focused Attitudes towards Mental Health Problems in Asian and Non-Asian Student Women. Mental Health, Religion \& Culture, 10(2), 127-141.

Gilbert, P., Gilbert, J., \& Sanghera, J. (2004). A Focus Group Exploration of the Impact of Izzat, Shame, Subordination and Entrapment on Mental Health and Service Use in South Asian Women Living in Derby. Mental Health, Religion and Culture, 7(2), 109-130.

Giner-Sorolla, R. (2001). Guilty Pleasures and Grim Necessities: Affective Attitudes in Dilemmas of Self-Control. Journal of Personality and Social Psychology, 80(2), 206-221. 
Goetz, J. L., \& Keltner, D. (2007). Shifting Meanings of Self-Conscious Emotions across Cultures. In J. L. Tracy, R. W. Robbins, \& J. P. Tangney (Eds.), The SelfConscious Emotions: Theory and Research (pp. 153-173). New York: Guilford Press.

Goss, K., \& Allan, S. (2009). Shame, Pride and Eating Disorders. Clinical Psychology and Psychotherapy, 16(4), 303-316.

Grabhorn, R., Stenner, H., Stangier, U., \& Kaufhold, J. (2006). Social Anxiety in Anorexia and Bulimia Nervosa: The Mediating Role of Shame. Clinical Psychology and Psychotherapy, 13(1), 12-19.

Graton, A., Ric, F., \& Gonzalez, E. (2016). Reparation or Reactance? The Influence of Guilt on Reaction to Persuasive Communication. Journal of Experimental Social Psychology, 62, 40-49.

Gregory, G. H. (2008). Questionnaire Design. In J. L. Paul (Ed.), Encyclopedia of Survey Research Methods (pp. 657-660). Thousand Oaks, CA: Sage Publications.

Grewal, R., Cote, J. A., \& Baumgartner, H. (2004). Multicollinearity and Measurement Error in Structural Equation Models: Implications for Theory Testing. Marketing Science, 23(4), 519-529.

Grol, R., \& Grimshaw, J. (2003). From Best Evidence to Best Practice: Effective Implementation of Change in Patients' Care. The Lancet, 362(9391), 1225-1230.

Gross, J. J. (1998). The Emerging Field of Emotion Regulation: An Integrative Review. Review of General Psychology, 2(3), 271-299.

Guba, E. G. (1990). The Paradigm Dialog. Newbury Park, California: Sage Publications.

Guba, E. G., \& Lincoln, Y. S. (1994). Competing Paradigms in Qualitative Research. In N. K. Denzin \& Y. S. Lincoln (Eds.), Handbook of Qualitative Research (pp. 105117). Thousand Oaks, CA: Sage Publications.

Hair, J. F., Anderson, R. E., Tatham, R. L., \& Black, W. C. (1998). Multivariate Data Analysis. Upper Saddle River, NJ: Prentice-Hall.

Hair, J. F., Black, W. C., Babin, B. J., \& Anderson, R. E. (2010). Multivariate Data Analysis (7th ed.). Upper Saddle River, NJ: Pearson-Prentice Hall.

Hair, J. F., Bush, R. P., \& Ortinau, D. J. (2009). Marketing Research: In a Digital Information Environment (4nd ed.). Chicago, IL: McGraw-Hill/Irwin Publisher.

Hair, J. F., Sarstedt, M., Ringle, C. M., \& Mena, J. A. (2012). An Assessment of the Use of Partial Least Squares Structural Equation Modeling in Marketing Research. Journal of the Academy of Marketing Science, 40(3), 414-433. 
Han, D., Duhachek, A., \& Agrawal, N. (2014). Emotions Shape Decisions through Construal Level: The Case of Guilt And Shame. Journal of Consumer Research, 41(4), 1047-1064.

Hanna, R., Rohm, A., \& Crittenden, V. L. (2011). We're All Connected: The Power of the Social Media Ecosystem. Business Horizons, 54(3), 265-273.

Hanson, D., \& Grimmer, M. (2007). The Mix of Qualitative and Quantitative Research in Major Marketing Journals, 1993-2002. European Journal of Marketing, 41(1/2), 58-70.

Hardin, E. E., Varghese, F. P., Tran, U. V., \& Carlson, A. Z. (2006). Anxiety and Career Exploration: Gender Differences in the Role of Self-Construal. Journal of Vocational Behavior, 69(2), 346-358.

Harwell, M. R., \& Gatti, G. G. (2001). Rescaling Ordinal Data to Interval Data in Educational Research. Review of Educational Research, 71(1), 105-131.

Hauben, M. (1997). The Net and Netizens: The Impact the Net Has on People's Lives. In M. Hauben \& R. Hauben (Eds.), Netizens: On the History and Impact of Usenet and the Internet. Milton QLD: Wiley-IEEE Computer Society Press.

Heine, S. J., Kitayama, S., Lehman, D. R., Takata, T., Ide, E., Leung, C., \& Matsumoto, H. (2001). Divergent Consequences of Success and Failure in Japan and North America: An Investigation of Self-Improving Motivations and Malleable Selves. Journal of Personality and Social Psychology, 81(4), 599-615.

Henseler, J., Ringle, C. M., \& Sarstedt, M. (2015). A New Criterion for Assessing Discriminant Validity in Variance-Based Structural Equation Modeling. Journal of the Academy of Marketing Science, 43(1), 115-135.

Hershberger, S. L. (2003). The Growth of Structural Equation Modeling: 1994-2001. Structural Equation Modeling, 10(1), 35-46.

Hibbert, S., Smith, A., Davies, A., \& Ireland, F. (2007). Guilt Appeals: Persuasion Knowledge and Charitable Giving. Psychology and Marketing, 24(8), 723-742.

Higgins, E. T. (1997). Beyond Pleasure and Pain. American Psychologist, 52(12), 12801300.

Higgins, E. T. (1998). Promotion and Prevention: Regulatory Focus as a Motivational Principle. In P. Z. Mark (Ed.), Advances in Experimental Social Psychology (Vol. 30, pp. 1-46). London, England: Academic Press. 
Higgins, E. T., Roney, C. J. R., Crowe, E., \& Hymes, C. (1994). Ideal versus Ought Predilections for Approach and Avoidance Distinct Self-Regulatory Systems. Journal of Personality and Social Psychology, 66(2), 276-286.

Higgins, E. T., Shah, J., \& Friedman, R. (1997). Emotional Responses to Goal Attainment: Strength of Regulatory Focus as Moderator. Journal of Personality and Social Psychology, 72(3), 515-525.

Hill, R. (2001). The Marketing Concept and Health Promotion: A Survey and Analysis of Recent Health Promotion Literature. Social Marketing Quarterly, 7(1), 29-53.

Hoblitzelle, W. (1987). Differentiating and Measuring Shame and Guilt: The Relation between Shame and Depression. In H. B. Lewis (Ed.), The Role of Shame in Symptom Formation (pp. 207-235). Hillsdale, NJ: Erlbaum.

Hoe, S. L. (2008). Issues and Procedures in Adopting Structural Equation Modeling Technique. Journal of Applied Quantitative Methods, 3(1), 76-83.

Hoelter, J. W. (1983). The Analysis of Covariance Structures Goodness-of-Fit Indices. Sociological Methods and Research, 11(3), 325-344.

Hofstede, G. (2001). Culture's Consequences: Comparing Values, Behaviors, Institutions and Organizations across Nations (2nd ed.). Thousand Oaks, CA: Sage Publications.

Hoglund, C. L., \& Nicholas, K. B. (1995). Shame, Guilt, and Anger in College Students Exposed to Abusive Family Environments. Journal of Family Violence, 10(2), 141-157.

Hox, J., \& Bechger, T. (1998). An Introduction to Structural Equation Modeling. Family Science Review, 11, 354-373.

Hu, L. t., \& Bentler, P. M. (1999). Cutoff Criteria for Fit Indexes in Covariance Structure Analysis: Conventional Criteria versus New Alternatives. Structural Equation Modeling: A Multidisciplinary Journal, 6(1), 1-55.

Huhmann, B. A., \& Brotherton, T. P. (1997). A Content Analysis of Guilt Appeals in Popular Magazine Advertisements. Journal of Advertising, 26(2), 35-45.

Hutton, F. (2012). Harm Reduction, Students and Pleasure: An Examination of Student Responses to a Binge Drinking Campaign. International Journal of Drug Policy, 23(3), 229-235.

Iacobucci, D. (2009). Everything You Always Wanted to Know about SEM (Structural Equations Modeling) But Were Afraid to Ask. Journal of Consumer Psychology, 19(4), 673-680. 
Izard, C. E. (1977). Human Emotions. New York: Plenum Press.

Jefferis, B., Power, C., \& Manor, O. (2005). Adolescent Drinking Level and Adult Binge Drinking in a National Birth Cohort. Addiction, 100(4), 543-549.

Jilke, S., Petrovsky, N., Meuleman, B., \& James, O. (2016). Measurement Equivalence in Replications of Experiments: When and Why It Matters and Guidance on How to Determine Equivalence. Public Management Review, 1-18.

Johnston, K. L., \& White, K. M. (2003). Binge-Drinking: A Test of the Role of Group Norms in the Theory of Planned Behaviour. Psychology and Health, 18(1), 63-77. Jöreskog, K. G. (1993). Testing Structural Equation Models. In K. A. Bollen \& J. S. Long (Eds.), Testing Structural Equation Models (pp. 294-316). Newbury Park, CA: Sage Publications.

Kagan, J. (1981). The Second Year: The Emergence of Self-Awareness. Cambridge, MA: Harvard University Press.

Karam, E., Kypri, K., \& Salamoun, M. (2007). Alcohol Use among College Students: An International Perspective. Current Opinion in Psychiatry, 20(3), 213-221.

Kaufman, G. (1996). The Psychology of Shame: Theory and Treatment of Shame-Based Syndromes (2nd ed.). New York: Springer Publishing Company.

Kees, J., Burton, S., \& Tangari, A. H. (2010). The Impact of Regulatory Focus, Temporal Orientation, and Fit on Consumer Responses to Health-Related Advertising. Journal of Advertising, 39(1), 19-34.

Keller, P. A., \& Lehmann, D. R. (2008). Designing Effective Health Communications: A Meta-Analysis. Journal of Public Policy and Marketing, 27(2), 117-130.

Keller, P. A., Lipkus, I. M., \& Rimer, B. K. (2003). Affect, Framing, and Persuasion. Journal of Marketing Research, 40(1), 54-64.

Keltner, D., \& Buswell, B. N. (1996). Evidence for the Distinctness of Embarrassment, Shame, and Guilt: A Study of Recalled Antecedents and Facial Expressions of Emotion. Cognition \& Emotion, 10(2), 155-172.

Keltner, D., \& Haidt, J. (1999). Social Functions of Emotions at Four Levels of Analysis. Cognition \& Emotion, 13(5), 505-521.

Keltner, D., Young, R. C., \& Buswell, B. N. (1997). Appeasement in Human Emotion, Social Practice, and Personality. Aggressive Behavior, 23(5), 359-374.

Kenny, D. A. (2014). Path Analysis. Structural Equation Modeling. Retrieved from http://davidakenny.net/cm/pathanal.htm 
Kim, J.-E., \& Johnson, K. K. P. (2013). The Impact of Moral Emotions on Cause-Related Marketing Campaigns: A Cross-Cultural Examination. Journal of Business Ethics, 112(1), 79-90.

Kim, J.-E., \& Johnson, K. K. P. (2014). Shame or Pride? The Moderating Role of SelfConstrual on Moral Judgments Concerning Fashion Counterfeits. European Journal of Marketing, 48(7/8), 1431-1450.

Kim, S., Thibodeau, R., \& Jorgensen, R. S. (2011). Shame, Guilt, and Depressive Symptoms: A Meta-Analytic Review. Psychological Bulletin, 137(1), 68-96.

Kim, Y., Kasser, T., \& Lee, H. (2003). Self-Concept, Aspirations, and Well-Being in South Korea and the United States. The Journal of Social Psychology, 143(3), 277-290.

Kirmani, A., \& Zhu, R. (2007). Vigilant against Manipulation: The Effect of Regulatory Focus on the Use of Persuasion Knowledge. Journal of Marketing Research, 44(4), 688-701.

Kitayama, S., Markus, H. R., \& Kurokawa, M. (2000). Culture, Emotion, and WellBeing: Good Feelings in Japan and the United States. Cognition and Emotion, 14(1), 93-124.

Kitayama, S., Markus, H. R., \& Matsumoto, H. (1995). Culture, Self, and Emotion: A Cultural Perspective on "Self-Conscious" Emotions. In J. P. Tangney \& K. W. Fischer (Eds.), Self-Conscious Emotions: The Psychology of Shame, Guilt, Embarrassment, and Pride (pp. 439-464). New York: Guilford Press.

Kitayama, S., Mesquita, B., \& Karasawa, M. (2006). Cultural Affordances and Emotional Experience: Socially Engaging and Disengaging Emotions in Japan and the United States. Journal of Personality and Social Psychology, 91(5), 890-903.

Kitayama, S., \& Park, H. (2007). Cultural Shaping of Self, Emotion, and Well-Being: How Does It Work? Social and Personality Psychology Compass, 1(1), 202-222.

Kitayama, S., Snibbe, A. C., Markus, H. R., \& Suzuki, T. (2004). Is There Any "Free" Choice? Self and Dissonance in Two Cultures. Psychological Science, 15(8), 527533.

Kline, R. B. (2016). Principles and Practice of Structural Equation Modeling (4th ed.). New York: Guilford Press.

Kondo, D. K. (1990). Crafting Selves: Power, Gender, and Discourses of Identity in a Japanese Workplace. Chicago: University of Chicago Press. 
Koufteros, X. A. (1999). Testing a Model of Pull Production: A Paradigm for Manufacturing Research Using Structural Equation Modeling. Journal of Operations Management, 17(4), 467-488.

Kühberger, A. (1998). The Influence of Framing on Risky Decisions: A Meta-Analysis. Organizational Behavior and Human Decision Processes, 75(1), 23-55.

Kuhn, T. S. (2012). The Structure of Scientific Revolutions (4th ed.). Chicago, IL: University of Chicago Press.

Kwan, V. S., Bond, M. H., \& Singelis, T. M. (1997). Pancultural Explanations for Life Satisfaction: Adding Relationship Harmony to Self-Esteem. Journal of Personality and Social Psychology, 73(5), 1038-1051.

Kypri, K., Cronin, M., \& Wright, C. (2005). Do University Students Drink More Hazardously Than Their Non-Student Peers? Addiction, 100(5), 713-714.

Kypri, K., Paschall, M. J., Langley, J., Baxter, J., Cashell-Smith, M., \& Bourdeau, B. (2009). Drinking and Alcohol-Related Harm among New Zealand University Students: Findings From a National Web-Based Survey. Alcoholism: Clinical and Experimental Research, 33(2), 307-314.

LaBarge, M. C., \& Godek, J. (2006). Mothers, Food, Love and Career: The Four Major Guilt Groups? The Differential Effects of Guilt Appeals. Advances in Consumer Research, 33, 511.

Lam, C. S., Tsang, H., Chan, F., \& Corrigan, P. W. (2006). Chinese and American Perspectives on Stigma. Rehabilitation Education, 20(4), 269-279.

Lam, C. S., Tsang, H. W. H., Corrigan, P. W., Lee, Y.-T., Angell, B., Shi, K., . . . Larson, J. E. (2010). Chinese Lay Theory and Mental Illness Stigma: Implications for Research and Practices. Journal of Rehabilitation, 76(1), 28-34.

LaTour, M. S., Pitts, R. E., \& Snook-Luther, D. C. (1990). Female Nudity, Arousal, and Ad Response: An Experimental Investigation. Journal of Advertising, 19(4), 5162.

LaTour, M. S., \& Rotfeld, H. J. (1997). There Are Threats and (Maybe) Fear-Caused Arousal: Theory and Confusions of Appeals to Fear and Fear Arousal Itself. Journal of Advertising, 26(3), 45-59.

Lau, R. R., Hartman, K. A., \& Ware, J. E. (1986). Health as a Value: Methodological and Theoretical Considerations. Health Psychology, 5(1), 25-43.

Lazarus, R. S. (1991a). Emotion and Adaptation. New York: Oxford University Press. 
Lazarus, R. S. (1991b). Progress on a Cognitive-Motivational-Relational Theory of Emotion. American Psychologist, 46(8), 819-834.

Leary, M. R., \& Baumeister, R. F. (2000). The Nature and Function of Self-Esteem: Sociometer Theory. Psychological Review, 116(2), 365-383.

Lee, A. Y., \& Aaker, J. L. (2002). Approach and Avoidance: The Role of Perceived Risk and Framing in Persuasion. Advances in Consumer Research, 29, 309-310.

Lee, A. Y., \& Aaker, J. L. (2004). Bringing the Frame into Focus: The Influence of Regulatory Fit on Processing Fluency and Persuasion. Journal of Personality and Social Psychology, 86(2), 205-218.

Lee, D. A., Scragg, P., \& Turner, S. (2001). The Role of Shame and Guilt in Traumatic Events: A Clinical Model of Shame-Based and Guilt-Based PTSD. British Journal of Medical Psychology, 74(4), 451-466.

Lee, H., \& Paek, H.-J. (2014). Roles of Guilt and Culture in Normative Influence: Testing Moderated Mediation in the Anti-Secondhand Smoking Context. Psychology, Health and Medicine, 19(1), 1-10.

Lee, N. R., \& Kotler, P. (2016). Social Marketing: Changing Behaviors for Good (5th ed.). Thousand Oaks, CA: Sage Publications.

Leshner, G., Bolls, P., \& Thomas, E. (2009). Scare'em or Disgust'em: The Effects of Graphic Health Promotion Messages. Health Communication, 24(5), 447-458.

Levenson, R. W. (1999). The Intrapersonal Functions of Emotion. Cognition and Emotion, 13(5), 481-504.

Levine, T. R., Bresnahan, M. J., Park, H. S., Lapinski, M. K., Wittenbaum, G. M., Shearman, S. M., . . . Ohashi, R. (2003). Self-Construal Scales Lack Validity. Human Communication Research, 29(2), 210-252.

Levinson, C. A., Langer, J. K., \& Rodebaugh, T. L. (2011). Self-Construal and Social Anxiety: Considering Personality. Personality and Individual Differences, 51(3), 355-359.

Lewis, H. B. (1971). Shame and Guilt in Neurosis. New York: International Universities Press.

Lewis, M. (1992). Shame: The Exposed Self. New York: Free Press.

Lewis, M. (1995). The Socialization of Shame: From Parent to Child. In M. Lewis (Ed.), Shame: The Exposed Self. New York: Free Press.

Lewis, M. (2000). The Emergence of Human Emotions. In M. Lewis \& J. Haviland-Jones (Eds.), Handbook of Emotions (pp. 265-280). New York: Guildford Press. 
Lewis, M., \& Brooks-Gunn, J. (1979). Social Cognition and the Acquisition of Self. New York: Plenum Press.

Li, J., Wang, L., \& Fischer, K. (2004). The Organization of Chinese Shame Concepts. Cognition and Emotion, 18, 767-797.

Lietz, P. (2010). Research into Questionnaire Design. International Journal of Market Research, 52(2), 249-272.

Lill, D., Gross, C., \& Peterson, R. (1986). The Inclusion of Social-Responsibility Themes by Magazine Advertisers: A Longitudinal Study. Journal of Advertising, 15(2), $35-41$.

Lindell, M. K., \& Whitney, D. J. (2001). Accounting for Common Method Variance in Cross-Sectional Research Designs. Journal of Applied Psychology, 86(1), 114121.

Lindsay-Hartz, J. (1984). Contrasting Experiences of Shame and Guilt. American Behavioral Scientist, 27, 689-704.

Lindsay-Hartz, J., de Rivera, J., \& Mascolo, M. F. (1995). Differentiating Guilt and Shame and Their Effects on Motivation. In J. P. Tangney \& K. W. Fischer (Eds.), Self-Conscious Emotions: Shame, Guilt, Embarrassment, and Pride (pp. 274300). New York: Guilford.

Lindsey, L. L. M. (2005). Anticipated Guilt as Behavioral Motivation: An Examination of Appeals to Help Unknown Others through Bone Marrow Donation. Human Communication Research, 31(4), 453-481.

Little, T. D., Cunningham, W. A., Shahar, G., \& Widaman, K. F. (2002). To Parcel or Not to Parcel: Exploring the Question, Weighing the Merits. Structural Equation Modeling, 9(2), 151-173.

Lockwood, P., Jordan, C. H., \& Kunda, Z. (2002). Motivation by Positive or Negative Role Models: Regulatory Focus Determines Who Will Best Inspire Us. Journal of Personality and Social Psychology, 83(4), 854-864.

Logan, D. E., Kilmer, J. R., \& Marlatt, G. A. (2010). The Virtuous Drinker: Character Virtues as Correlates and Moderators of College Student Drinking and Consequences. Journal of American College Health, 58(4), 317-324.

Lord, C. G. (1980). Schemas and Images as Memory Aids: Two Modes of Processing Social Information. Journal of Personality and Social Psychology, 38, 257-269. 
Lu, L., \& Gilmour, R. (2007). Developing a New Measure of Independent and Interdependent Views of the Self. Journal of Research in Personality, 41(1), 249257.

Lutwak, N., Razzino, B. E., \& Ferrari, J. R. (1998). Self-Perceptions and Moral Affect: An Exploratory Analysis of Subcultural Diversity in Guilt and Shame Emotions. Journal of Social Behavior and Personality, 13(2), 333-348.

Lwin, M., \& Phau, I. (2010). Conceptualising Anticipatory Guilt in a Non-Durable Consumer Goods Context. Paper presented at the Recent Advances in Retailing and Services Science Conference, Istanbul, Turkey.

Lwin, M., \& Phau, I. (2011). The Role of Guilt Appeals on Donation Behavior. Paper presented at the 20th World Business Congress, Poznan, Poland.

Lwin, M., \& Phau, I. (2014). An Exploratory Study of Existential Guilt Appeals in Charitable Advertisements. Journal of Marketing Management, 30(13-14), 14671485.

Lynd, H. M. (1958). On Shame and the Search for Identity. Oxon, England: Routledge.

MacKinnon, D. P., Krull, J. L., \& Lockwood, C. M. (2000). Equivalence of the Mediation, Confounding and Suppression Effect. Prevention Science, 1(4), 173181.

MacKinnon, D. P., Lockwood, C. M., Hoffman, J. M., West, S. G., \& Sheets, V. (2002). A Comparison of Methods to Test Mediation and Other Intervening Variable Effects. Psychological Methods, 7(1), 83.

MacKinnon, D. P., Lockwood, C. M., \& Williams, J. (2004). Confidence Limits for the Indirect Effect: Distribution of the Product and Resampling Methods. Multivariate Behavioral Research, 39(1), 99-128.

Maibach, E. W., Abroms, L. C., \& Marosits, M. (2007). Communication and Marketing as Tools to Cultivate the Public's Health: A Proposed "People and Places" Framework. BMC Public Health, 7, 88-102.

Mangleburg, T. F., \& Bristol, T. (1998). Socialization and Adolescents' Skepticism toward Advertising. Journal of Advertising, 27(3), 11-21.

Markus, H. R., \& Kitayama, S. (1991). Culture and the Self: Implications for Cognition, Emotion, and Motivation. Psychological Review, 98(2), 224-253.

Marquis, K. H., Marquis, M. S., \& Polich, J. M. (1986). Response Bias and Reliability in Sensitive Topic Surveys. Journal of the American Statistical Association, 81(394), 381-389. 
Marsh, H. W., Hau, K.-T., \& Wen, Z. (2004). In Search of Golden Rules: Comment on Hypothesis-Testing Approaches to Setting Cutoff Values for Fit Indexes and Dangers in Overgeneralizing $\mathrm{Hu}$ and Bentler's (1999) Findings. Structural Equation Modeling, 11(3), 320-341.

Martin, B. A., Gnoth, J., \& Strong, C. (2009). Temporal Construal in Advertising: The Moderating Role of Temporal Orientation and Attribute Importance upon Consumer Evaluations. Journal of Advertising, 38(3), 5-19.

Martin, B. A. S., Lee, C. K.-C., Weeks, C., \& Kaya, M. (2013). How to Stop Binge Drinking and Speeding Motorists: Effects of Relational-Interdependent SelfConstrual and Self-Referencing on Attitudes toward Social Marketing. Journal of Consumer Behaviour, 12(1), 81-90.

Martin, B. A. S., Lee, C. K.-C., \& Yang, F. (2004). The Influence of Ad Model Ethnicity and Self-Referencing on Attitudes: Evidence from New Zealand. Journal of Advertising, 33(4), 27-37.

McDonald, R. P., \& Ho, M.-H. R. (2002). Principles and Practice in Reporting Structural Equation Analyses. Psychological Methods, 7(1), 64-82.

McGee, R., \& Kypri, K. (2004). Alcohol-Related Problems Experienced by University Students in New Zealand. Australian and New Zealand Journal of Public Health, 28(4), 321-323.

Mead, M. (1971). Some Anthropological Considerations Concerning Guilt. In R. W. Smith (Ed.), Guilt: Man and Society. New York: Anchor Books.

Menon, U., \& Shweder, R. A. (1994). Kali's Tongue: Cultural Psychology and the Power of Shame in Orissa, India. In S. Kitayama \& H. R. Markus (Eds.), Emotion and Culture: Empirical Studies of Mutual Influence (pp. 241-282). Washington, DC: American Psychological Association.

Meredith, W. (1993). Measurement Invariance, Factor Analysis and Factorial Invariance. Psychometrika, 58(4), 525-543.

Mesquita, B. (2001). Emotions in Collectivist and Individualist Contexts. Journal of Personality and Social Psychology, 80(1), 68-74.

Mesquita, B., \& Karasawa, M. (2004). Self-Conscious Emotions as Dynamic Cultural Processes. Psychological Inquiry, 15, 161-166.

Meyers-Levy, J., \& Peracchio, L. A. (1996). Moderators of the Impact of Self-Reference on Persuasion. Journal of Consumer Research, 22(4), 408-423. 
Miller, M. (2012). Shame and Psychotherapy. Retrieved March 15, 2014, from Columbia Psychotherapy Associates http://www.columbiapsych.com/shame_miller.html

Mills, R. S. (2005). Taking Stock of the Developmental Literature on Shame. Developmental Review, 25(1), 26-63.

Mittal, V., Kumar, P., \& Tsiros, M. (1999). Attribute-Level Performance, Satisfaction, and Behavioral Intentions over Time: A Consumption-System Approach. The Journal of Marketing, 63(2), 88-101.

Moore, D. J., \& Harris, W. D. (1996). Affect Intensity and the Consumer's Attitude toward High Impact Emotional Advertising Appeals. Journal of Advertising, 25(2), 37-50.

Moorman, C. (1994). An Innovation Adoption Approach to the Dissemination of Health Information to Consumers. In L. Secrest, T. E. Becker, E. M. Rogers, T. F. Campbell, \& M. L. Grady (Eds.), Effective Dissemination of Clinical and Health Information (pp. 49-68). Washington, DC: Agency for Health Care Policy Research.

Murray-Johnson, L., Witte, K., Liu, W., Hubbell, A. P., Sampson, J., \& Morrison, K. (2001). Addressing Cultural Orientations in Fear Appeals: Promoting AidsProtective Behaviors among Mexican Immigrant and African American Adolescents and American and Taiwanese College Students. Journal of Health Communication, 6(4), 335-358.

Na, J., Grossmann, I., Varnum, M. E., Kitayama, S., Gonzalez, R., \& Nisbett, R. E. (2010). Cultural Differences Are Not Always Reducible to Individual Differences. Proceedings of the National Academy of Sciences, 107(14), 6192-6197.

New Zealand Ministry of Health. (2015). Alcohol Use 2012/13: New Zealand Health Survey. Wellington, New Zealand: Ministry of Health. Retrieved from http://www.health.govt.nz/system/files/documents/publications/alcohol-use-201213-new-zealand-health-survey-feb15.pdf.

Niederdeppe, J., Bu, Q., Borah, P., Kindig, D. A., \& Robert, S. A. (2008). Message Design Strategies to Raise Public Awareness of Social Determinants of Health and Population Health Disparities. Milbank Quarterly, 86(3), 481-513.

Noble, G. I., \& Camit, M. (2005). Social Marketing Communication in a Multicultural Environment: Practical Issues and Theoretical Contributions from Cross-Cultural Marketing. PRism, 3(2), 1-13. 
Norman, P., \& Smith, L. (1995). The Theory of Planned Behaviour and Exercise: An Investigation into the Role of Prior Behaviour, Behavioural Intentions and Attitude Variability. European Journal of Social Psychology, 25(4), 403-415.

O'Keefe, D. J. (2000). Guilt and Social Influence. In M. Roloff (Ed.), Communication Yearbook (Vol. 23, pp. 67-101). Thousand Oaks, CA: Sage Publications.

O'Keefe, D. J. (2002). Guilt as a Mechanism of Persuasion. In P. D. James \& P. Michael (Eds.), The Persuasion Handbook: Developments in Theory and Practice (pp. 329-344). Thousand Oaks: Sage Publications.

O'Leary, Z. (2013). The Essential Guide to Doing Your Research Project (2nd ed.). London, England: Sage Publications.

O'Malley, P. M., Johnston, L. D., \& Bachman, J. G. (1998). Alcohol Use among Adolescents. Alcohol Research and Health World, 22(2), 85-93.

Orth, U., Robins, R. W., \& Soto, C. J. (2010). Tracking the Trajectory of Shame, Guilt, and Pride across the Life Span. Journal of Personality and Social Psychology, 99(6), 1061-1071.

Park, I. J. K., Sulaiman, C., Schwartz, S. J., Kim, S. Y., Ham, L. S., \& Zamboanga, B. L. (2011). Self-Construals and Social Anxiety among Asian American College Students: Testing Emotion Suppression as a Mediator. Asian American Journal of Psychology, 2(1), 39-50.

Parrott, R. (1995). Designing Messages for Behavioral Inoculation. In R. L. Parrott \& E. Maibach (Eds.), Designing Health Messages: Approaches from Communication Theory and Public Health Practice (pp. 99-113). Beverly Hills, CA: Sage Publications.

Paul, C. L., Redman, S., \& Sanson-Fisher, R. W. (1997). The Development of a Checklist of Content and Design Characteristics for Printed Health Education Materials. Health Promotion Journal of Australia, 7(3), 153-159.

Pechmann, C. (2001). A Comparison of Health Communication Models: Risk Learning versus Stereotype Priming. Media Psychology, 3(2), 189-210.

Pechmann, C., Zhao, G., Goldberg, M. E., \& Reibling, E. T. (2003). What to Convey in Antismoking Advertisements for Adolescents: The Use of Protection Motivation Theory to Identify Effective Message Themes. Journal of Marketing, 67(2), 1-18.

Piers, G., \& Singer, M. B. (1971). Shame and Guilt: A Psychoanalytic and a Cultural Study. Oxford, England: W. W. Norton. 
Pinto, M. B., \& Priest, S. (1991). Guilt Appeals in Advertising: An Exploratory Study. Psychological Reports, 69(2), 375-385.

Pinto, M. B., \& Worobetz, N. D. (1992). Note on Guilt Appeals in Advertising: Covariate Effects of Self-Esteem and Locus of Control. Psychological Reports, 70(1), 19-22. Plutchik, R. (1980). A General Psychoevolutionary Theory of Emotion. In R. Plutchik \& H. Kellerman (Eds.), Emotion: Theory, Research, and Experience (Vol. 1, pp. 331). New York: Academic Press.

Podsakoff, P. M., MacKenzie, S. B., Lee, J.-Y., \& Podsakoff, N. P. (2003). Common Method Biases in Behavioral Research: A Critical Review of the Literature and Recommended Remedies. Journal of Applied Psychology, 88(5), 879-903.

Poynter, R. (2001). A Guide to Best Practice in Online Quantitative Research. Paper presented at the International Conference on Survey Research Methods, Chesham, UK.

Preacher, K. J., \& Hayes, A. F. (2008a). Assessing Mediation in Communication Research. In A. F. Hayes, M. D. Slater, \& L. B. Snyder (Eds.), The Sage Sourcebook of Advanced Data Analysis Methods for Communication Research (pp. 13-54). Thousand Oaks, CA: Sage Publications.

Preacher, K. J., \& Hayes, A. F. (2008b). Asymptotic and Resampling Strategies for Assessing and Comparing Indirect Effects in Multiple Mediator Models. Behavior Research Methods, 40(3), 879-891.

Preacher, K. J., \& Leonardelli, G. J. (2010). Calculation for the Sobel Test: An Interactive Calculation Tool for Mediation Tests. Retrieved from http://quantpsy.org/sobel/sobel.htm

Proeve, M., \& Howells, K. (2002). Shame and Guilt in Child Sexual Offenders. International Journal of Offender Therapy and Comparative Criminology, 46(6), 657-667.

Rawlings, E. I. (1970). Reactive Guilt and Anticipatory Guilt in Altruistic Behavior. In J. R. Macaulay \& L. Berkowitz (Eds.), Altruism and Helping Behavior (pp. 163177). New York: Academic Press.

Rea, L. M., \& Parker, R. A. (2005). Designing and Conducting Survey Research: A Comprehensive Guide (3rd ed.). San Francisco, CA: John Wiley \& Sons.

Read, J. P., Kahler, C. W., Strong, D. R., \& Colder, C. R. (2006). Development and Preliminary Validation of the Young Adult Alcohol Consequences Questionnaire. Journal of Studies on Alcohol and Drugs, 67(1), 169-177. 
Redman, S., \& Paul, C. L. (1997). A Review of the Effectiveness of Print Material in Changing Health-Related Knowledge, Attitudes and Behaviour. Health Promotion Journal of Australia, 7(2), 91-99.

Rehm, J., Mathers, C., Popova, S., Thavorncharoensap, M., Teerawattananon, Y., \& Patra, J. (2009). Global Burden of Disease and Injury and Economic Cost Attributable to Alcohol Use and Alcohol-Use Disorders. The Lancet, 373(9682), 2223-2233.

Rehm, J., Room, R., Graham, K., Monteiro, M., Gmel, G., \& Sempos, C. T. (2003). The Relationship of Average Volume of Alcohol Consumption and Patterns of Drinking to Burden of Disease: An Overview. Addiction, 98(9), 1209-1228.

Reinert, D. F., \& Allen, J. P. (2007). The Alcohol Use Disorders Identification Test: An Update of Research Findings. Alcoholism: Clinical and Experimental Research, 31(2), 185-199.

Reinhart, A. M., Marshall, H. M., Feeley, T. H., \& Tutzauer, F. (2007). The Persuasive Effects of Message Framing in Organ Donation: The Mediating Role of Psychological Reactance. Communication Monographs, 74(2), 229-255.

Renner, S., Lindenmeier, J., Tscheulin, D. K., \& Drevs, F. (2013). Guilt Appeals and Prosocial Behavior: An Experimental Analysis of the Effects of Anticipatory versus Reactive Guilt Appeals on the Effectiveness of Blood Donor Appeals. Journal of Nonprofit \& Public Sector Marketing, 25(3), 237-255.

Ridout, T. N., Shah, D. V., Goldstein, K. M., \& Franz, M. M. (2004). Evaluating Measures of Campaign Advertising Exposure on Political Learning. Political Behavior, 26(3), 201-225.

Rogers, R. W. (1975). A Protection Motivation Theory of Fear Appeals and Attitude Change. The Journal of Psychology, 91(1), 93-114.

Rogers, R. W. (1983). Cognitive and Physiological Processes in Fear Appeals and Attitude Change: A Revised Theory of Protection Motivation. In J. Cacioppo \& R. Petty (Eds.), Social Psychophysiology (pp. 153-176). New York: Guilford Press.

Rönkkö, M., \& Ylitalo, J. (2011). PLS Marker Variable Approach to Diagnosing and Controlling for Method Variance. Paper presented at the Thirty Second International Conference on Information Systems, Shanghai.

Roper, W. L. (1993). Health Communication Takes on New Dimensions at CDC. Public Health Reports, 108(2), 179-183. 
Roser, C. (1990). Involvement, Attention, and Perceptions of Message Relevance in the Response to Persuasive Appeals. Communication Research, 17(5), 571-600.

Rucker, D. D., Preacher, K. J., Tormala, Z. L., \& Petty, R. E. (2011). Mediation Analysis in Social Psychology: Current Practices and New Recommendations. Social and Personality Psychology Compass, 5(6), 359-371.

Sabini, J., \& Silver, M. (1997). In Defense of Shame: Shame in the Context of Guilt and Embarrassment. Journal for the Theory of Social Behaviour, 27(1), 1-15.

Saintives, C., \& Lunardo, R. (2016). How Guilt Affects Consumption Intention: The Role of Rumination, Emotional Support and Shame. Journal of Consumer Marketing, $33(1), 41-51$.

Sale, J. E., Lohfeld, L. H., \& Brazil, K. (2002). Revisiting the Quantitative-Qualitative Debate: Implications for Mixed-Methods Research. Quality and Quantity, 36(1), 43-53.

Schaefer, A. D., Hermans, C. M., \& Parker, R. S. (2005). A Cross-Cultural Exploration of Advertising Skepticism and Media Effects in Teenagers. Marketing Management Journal, 15(2), 29-42.

Schoenbachler, D. D., \& Whittler, T. E. (1996). Adolescent Processing of Social and Physical Threat Communications. Journal of Advertising, 25(4), 37-54.

Schore, A. N. (1998). Early Shame Experiences and Infant Brain Development. In P. E. Gilbert \& B. E. Andrews (Eds.), Shame: Interpersonal Behavior, Psychopathology, and Culture. New York: Oxford University Press.

Schumacker, R. E., \& Lomax, R. G. (2004). A Beginner's Guide to Structural Equation Modeling (2nd ed.). Mahwah, NJ: Lawrence Erlbaum Associates.

Schumann, J. H., Wangenheim, F. v., Stringfellow, A., Yang, Z., Blazevic, V., Praxmarer, S., . . Jiménez, F. R. (2010). Cross-Cultural Differences in the Effect of Received Word-of-Mouth Referral in Relational Service Exchange. Journal of International Marketing, 18(3), 62-80.

Scollon, C. N., Koh, S., \& Au, E. W. M. (2011). Cultural Differences in the Subjective Experience of Emotion: When and Why They Occur. Social and Personality Psychology Compass, 5(11), 853-864.

Sedikides, C., \& Green, J. D. (2000). On the Self-Protective Nature of InconsistencyNegativity Management: Using the Person Memory Paradigm to Examine SelfReferent Memory. Journal of Personality and Social Psychology, 79(6), 906-922. 
Shrout, P. E., \& Bolger, N. (2002). Mediation in Experimental and Nonexperimental Studies: New Procedures and Recommendations. Psychological Methods, 7(4), $422-445$.

Silfver-Kuhalampi, M. (2009). The Sources of Moral Motivation: Studies on Empathy, Guilt, Shame and Values. (Doctoral dissertation), University of Helsinki.

Silfver, M. (2007). Coping with Guilt and Shame: A Narrative Approach. Journal of Moral Education, 36(2), 169-183.

Singelis, T. M. (1994). The Measurement of Independent and Interdependent SelfConstruals. Personality and Social Psychology Bulletin, 20(5), 580-591.

Singelis, T. M., \& Sharkey, W. F. (1995). Culture, Self-Construal, and Embarrassability. Journal of Cross-Cultural Psychology, 26(6), 622-644.

Sinh, N. H. (2013). Highly Attractive Models in Advertising: What Causes Negative Affect? Journal of International Business and Economy, 14(1), 31-48.

Sinh, N. H. (2014). Người mẫu cực hấp dẫn trong quảng cáo: Điều gì gây nên hiệu ứng tiêu cực? Tạp chí Phát triển Khoa học và Công nghệ (Science and Technology Development), 16(Q1), 20-34.

Slutske, W. S. (2005). Alcohol Use Disorders among US College Students and Their Non-College-Attending Peers. Archives of General Psychiatry, 62(3), 321-327.

Smith, M. S., Wallston, K. A., \& Smith, C. A. (1995). The Development and Validation of the Perceived Health Component Scale. Health Education Research, 10, 51-64.

Stark, J. H., \& Frenkel, D. N. (2013). Using Fear and Guilt to Persuade: What Might Empirical Research Tell Mediators? Dispute Resolution Magazine, 19(2), 26-29.

Steele, C. M. (1988). The Psychology of Self-Affirmation: Sustaining the Integrity of the Self. In L. Berkowitz (Ed.), Advances in Experimental Social Psychology (Vol. 21, pp. 261-302). New York: Academic Press.

Steenkamp, J.-B. E., \& Baumgartner, H. (1998). Assessing Measurement Invariance in Cross-National Consumer Research. Journal of Consumer Research, 25(1), 78-90.

Stiles, J. (2003). A Philosophical Justification for a Realist Approach to Strategic Alliance Research. Qualitative Market Research: An International Journal, 6(4), 263-271.

Stipek, D., Recchia, S., \& McClintic, S. (1992). Self-Evaluation in Young Children. Monographs of the Society for Research in Child Development, 57, 1-84. 
Stipek, D., Weiner, B., \& Li, K. (1989). Testing Some Attribution-Emotion Relations in the People's Republic of China. Journal of personality and social psychology, 56(1), 109-116.

Straus, S., Tetroe, J., \& Graham, I. D. (2011). Knowledge Translation in Health Care: Moving from Evidence to Practice. NJ: John Wiley \& Sons.

Streiner, D. L. (2005). Finding Our Way: An Introduction to Path Analysis. The Canadian Journal of Psychiatry, 50(2), 115-122.

Stuewig, J., \& McCloskey, L. A. (2005). The Relation of Child Maltreatment to Shame and Guilt among Adolescents: Psychological Routes to Depression and Delinquency. Child Maltreatment, 10(4), 324-336.

Stuewig, J., Tangney, J. P., Heigel, C., Harty, L., \& McCloskey, L. (2010). Shaming, Blaming, and Maiming: Functional Links among the Moral Emotions, Externalization of Blame, and Aggression. Journal of Research in Personality, 44(1), 91-102.

Symons, C. S., \& Johnson, B. T. (1997). The Self-Reference Effect in Memory: A MetaAnalysis. Psychological Bulletin, 121(3), 371-394.

Sznycer, D., Tooby, J., Cosmides, L., Porat, R., Shalvi, S., \& Halperin, E. (2016). Shame Closely Tracks the Threat of Devaluation by Others, Even across Cultures. Proceedings of the National Academy of Sciences, 113(10), 2625-2630.

Tangney, J. P. (1991). Moral Affect: The Good, the Bad, and the Ugly. Journal of Personality and Social Psychology, 61(4), 598-607.

Tangney, J. P. (1992). Situational Determinants of Shame and Guilt in Young Adulthood. Personality and Social Psychology Bulletin, 18, 199-206.

Tangney, J. P. (1993). Shame and guilt. In C. G. Costello (Ed.), Symptoms of depression (pp. 161-180). New York: Wiley.

Tangney, J. P. (1995). Shame and Guilt in Interpersonal Relationships. In J. P. Tangney \& K. W. Fisher (Eds.), Self-Conscious Emotions: The Psychology of Shame, Guilt, Embarrassment, and Pride (pp. 114-142). New York: Guildford Press.

Tangney, J. P. (1998). How Does Guilt Differ from Shame? In J. Bybee (Ed.), Guilt and Children (pp. 1-17). San Diego, CA: Academic Press.

Tangney, J. P. (1999). The Self-Conscious Emotions: Shame, Guilt, Embarrassment and Pride. In T. Dalgleish \& M. Power (Eds.), Handbook of Cognition and Emotion (pp. 541-568). New York: John Wiley \& Sons.

Tangney, J. P., \& Dearing, R. L. (2002). Shame and Guilt. New York: Guilford Press. 
Tangney, J. P., \& Fischer, K. (1995). Self-Conscious Emotions: The Psychology of Shame, Guilt, Embarrassment, and Pride. New York: Guilford Press.

Tangney, J. P., Miller, R. S., Flicker, L., \& Barlow, D. H. (1996). Are Shame, Guilt, and Embarrassment Distinct Emotions? Journal of Personality and Social Psychology, 70(6), 1256-1269.

Tangney, J. P., Stuewig, J., \& Mashek, D. J. (2007). Moral Emotions and Moral Behavior. Annual Review of Psychology, 58, 345-372.

Tangney, J. P., Wagner, P., Fletcher, C., \& Gramzow, R. (1992). Shamed into Anger? The Relation of Shame and Guilt to Anger and Self-Reported Aggression. Journal of Personality and Social Psychology, 62(4), 669-675.

Tangney, J. P., Wagner, P. E., Hill-Barlow, D., Marschall, D. E., \& Gramzow, R. (1996). Relation of Shame and Guilt to Constructive versus Destructive Responses to Anger across the Lifespan. Journal of Personality and Social Psychology, 70(4), 797-809.

Tanner, J., J. F., Hunt, J. B., \& Eppright, D. R. (1991). The Protection Motivation Model: A Normative Model of Fear Appeals. Journal of Marketing, 55(3), 36-45.

Taylor, S. E., \& Brown, J. D. (1988). Illusion and Well-Being: A Social Psychological Perspective on Mental Health. Psychological Bulletin, 103(2), 193-210.

Taylor, S. E., Kemeny, M. E., Reed, G. M., Bower, J. E., \& Gruenewald, T. L. (2000). Psychological Resources, Positive Illusions, and Health. American Psychologist, 55(1), 99-109.

Teroni, F., \& Deonna, J. A. (2008). Differentiating Shame from Guilt. Consciousness and Cognition, 17(3), 725-740.

Thomas, K. A., DeScioli, P., Haque, O. S., \& Pinker, S. (2014). The Psychology of Coordination and Common Knowledge. Journal of Personality and Social Psychology, 107(4), 657-676.

Thompson, B. (1998). Statistical Significance and Effect Size Reporting: Portrait of a Possible Future. Research in the Schools, 5(2), 33-38.

Tiedens, L. Z., \& Linton, S. (2001). Judgment under Emotional Certainty and Uncertainty: The Effects of Specific Emotions on Information Processing. Journal of Personality and Social Psychology, 81(6), 973-988.

Tinsley, C. H., \& Weldon, E. (2003). Responses to a Normative Conflict among American and Chinese Managers. International Journal of Cross Cultural Management, 3(2), 183-194. 
Tooby, J., \& Cosmides, L. (1990). The Past Explains the Present: Emotional Adaptations and the Structure of Ancestral Environments. Ethology and Sociobiology, 11(4), $375-424$.

Tracy, J. L., \& Robins, R. W. (2007a). Self-Conscious Emotions: Where Self and Emotion Meet. In C. Sedikides \& S. J. Spencer (Eds.), The Self: Frontiers of Social Psychology (pp. 187-209). New York: Psychology Press.

Tracy, J. L., \& Robins, R. W. (2007b). The Self in Self-Conscious Emotions: A Cognitive Appraisal Approach. In J. L. Tracy, R. W. Robins, \& J. P. Tangney (Eds.), The Self-Conscious Emotions: Theory and Research (pp. 3-20). New York: Guilford Press.

Trafimow, D., Triandis, H. C., \& Goto, S. G. (1991). Some Tests of the Distinction between the Private Self and the Collective Self. Journal of Personality and Social Psychology, 60(5), 649-655.

Treeby, M., \& Bruno, R. (2012). Shame and Guilt-Proneness: Divergent Implications for Problematic Alcohol Use and Drinking to Cope with Anxiety and Depression Symptomatology. Personality and Individual Differences, 53(5), 613-617.

Turner, M. M. (2012). Using Emotional Appeals in Health Messages. In C. Hyunyi (Ed.), Health Communication Message Design: Theory and Practice (pp. 59-72). Los Angeles, CA: Sage Publications.

Turner, M. M., \& Underhill, J. C. (2012). Motivating Emergency Preparedness Behaviors: The Differential Effects of Guilt Appeals and Actually Anticipating Guilty Feelings. Communication Quarterly, 60(4), 545-559.

van Hamersveld, M., \& de Bont, C. (2007). Market Research Handbook. West Sussex, England: John Wiley \& Sons.

Van Vliet, K. J. (2009). The Role of Attributions in the Process of Overcoming Shame: A Qualitative Analysis. Psychology and Psychotherapy: Theory, Research and Practice, 82(2), 137-152.

Vernon, S. W., McQueen, A., Tiro, J. A., \& Del Junco, D. J. (2010). Interventions to Promote Repeat Breast Cancer Screening with Mammography: A Systematic Review and Meta-Analysis. JNCI: Journal of the National Cancer Institute, 102(14), 1023-1039.

Victoria University of Wellington. (2016). Human Ethics Policy. Retrieved from http://www.victoria.ac.nz/documents/policy/research-policy/human-ethicspolicy.pdf 
Voorhees, C. M., Brady, M. K., Calantone, R., \& Ramirez, E. (2016). Discriminant Validity Testing in Marketing: An Analysis, Causes for Concern, and Proposed Remedies. Journal of the Academy of Marketing Science, 44(1), 119-134.

Wallbott, H. G., \& Scherer, K. R. (1995). Cultural Determinants in Experiencing Shame and Guilt. In J. P. Tangney \& K. W. Fischer (Eds.), Self-Consciuos Emotions: The Psychology of Shame, Guilt, Embarrassment, and Pride (pp. 465-487). New York: Guilford Press.

Wechsler, H., Lee, J. E., Kuo, M., Seibring, M., Nelson, T. F., \& Lee, H. (2002). Trends in College Binge Drinking during a Period of Increased Prevention Efforts: Findings from 4 Harvard School of Public Health College Alcohol Study Surveys: 1993-2001. Journal of American College Health, 50(5), 203-217.

Weiner, B. (1985). An Attributional Theory of Achievement Motivation and Emotion. Psychological Review, 92(4), 548-573.

Weiner, B. (1986). An Attributional Theory of Motivation and Emotion. New York: Springer.

Wicker, F. W., Payne, G. C., \& Morgan, R. D. (1983). Participant Descriptions of Guilt and Shame. Motivation and Emotion, 7(1), 25-39.

Williams, J., \& MacKinnon, D. P. (2008). Resampling and Distribution of the Product Methods for Testing Indirect Effects in Complex Models. Structural Equation Modeling, 15(1), 23-51.

Williams, L. J., Hartman, N., \& Cavazotte, F. (2010). Method Variance and Marker Variables: A Review and Comprehensive CFA Marker Technique. Organizational Research Methods, 13(3), 477-514.

Williams, P., \& Aaker, J. L. (2002). Can Mixed Emotions Peacefully Coexist? Journal of Consumer Research, 28(4), 636-649.

Wilson, E. A., Makoul, G., Bojarski, E. A., Bailey, S. C., Waite, K. R., Rapp, D. N., . . Wolf, M. S. (2012). Comparative Analysis of Print and Multimedia Health Materials: A Review of the Literature. Patient Education and Counseling, 89(1), 7-14.

Wilson, E. A., Park, D. C., Curtis, L. M., Cameron, K. A., Clayman, M. L., Makoul, G., . . . Wolf, M. S. (2010). Media and Memory: The Efficacy of Video and Print Materials for Promoting Patient Education about Asthma. Patient Education and Counseling, 80(3), 393-398. 
Windle, M. (2003). Alcohol Use among Adolescents and Young Adults. Population, 45(5.9), 19-15.

Wong, Y., \& Tsai, J. (2007). Cultural Models of Shame and Guilt. In J. Tracy, R. Robins, \& J. Tangney (Eds.), The Self-Conscious Emotions: Theory and Research (pp. 209-223). New York: Guilford Press.

World Health Organization. (2001). Global Status Report: Alcohol and Young People. Geneva, Switzerland: World Health Organization.

World Health Organization. (2002). The World Health Report 2002: Reducing Risks, Promoting Healthy Life. Geneva, Switzerland: World Health Organization.

World Health Organization. (2007). WHO Expert Committee on Problems Related to Alcohol Consumption. Geneva, Switzerland: World Health Organization.

World Health Organization. (2015). Alcohol. Fact sheet. Retrieved from http://www.who.int/mediacentre/factsheets/fs349/en/

Wright, K. B. (2005). Researching Internet-Based Populations: Advantages and Disadvantages of Online Survey Research, Online Questionnaire Authoring Software Packages, and Web Survey Services. Journal of Computer-Mediated Communication, 10(3), 00-00.

Xie, D., Leong, F. T., \& Feng, S. (2008). Culture-Specific Personality Correlates of Anxiety Among Chinese and Caucasian College Students. Asian Journal of Social Psychology, 11(2), 163-174.

Yoo, B. (2009). Cross-National Invariance of the Effect of Personal Collectivistic Orientation on Brand Loyalty and Equity: The United States versus South Korean Consumers. Asia Pacific Journal of Marketing and Logistics, 21(1), 41-57.

Yoo, B., \& Donthu, N. (2002). The Effects of Marketing Education and Individual Cultural Values on Marketing Ethics of Students. Journal of Marketing Education, 24(2), 92-103.

Yoo, B., \& Donthu, N. (2005). The Effect of Personal Cultural Orientation on Consumer Ethnocentrism: Evaluations and Behaviors of US Consumers toward Japanese Products. Journal of International Consumer Marketing, 18(1-2), 7-44.

Yu, N., \& Shen, F. (2012). Benefits for Me or Risks for Others: A Cross-Culture Investigation of the Effects of Message Frames and Cultural Appeals. Health Communication, 28(2), 133-145.

Zaichkowsky, J. L. (1985). Measuring the Involvement Construct. Journal of Consumer Research, 12(3), 341-352. 
Zhao, G., \& Pechmann, C. (2007). The Impact of Regulatory Focus on Adolescents' Response to Antismoking Advertising Campaigns. Journal of Marketing Research, 44(4), 671-687.

Zikmund, W. G., Ward, S., Winzar, H., Lowe, B., \& Babin, B. J. (2014). Marketing Research: Asia Pacific Edition (3rd ed.). South Melbourne, Victoria: Cengage Learning Australia.

Zumbo, B. D., Sireci, S. G., \& Hambleton, R. K. (2003). Re-Visiting Exploratory Methods for Construct Comparability: Is There Something to Be Gained from the Ways of Old. Paper presented at the Symposium Construct Comparability Research: Methodological Issues and Results, National Council on Measurement in Education. 


\section{Appendices}

\section{Appendix 1. Stimulus Advertisements}

\section{Self-referenced guilt, male image}

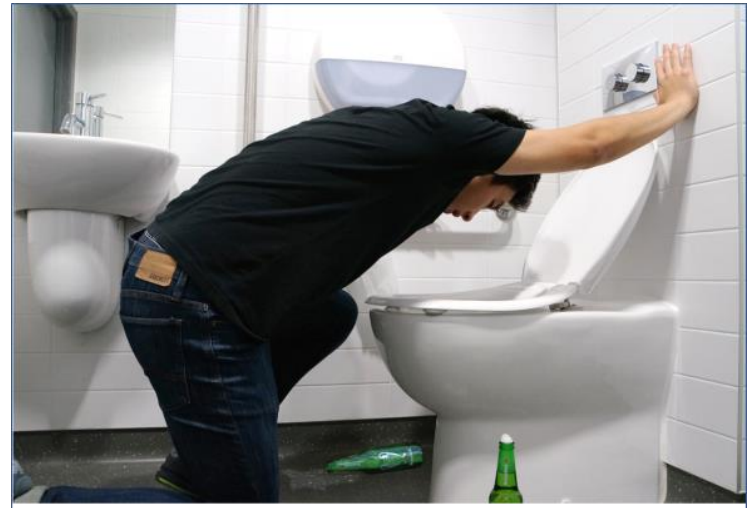

GUILTY Moment of Life

If YOU binge drink:

- You should feel GUILTY about your irresponsible drinking behaviour.

- You could get arrested due to your harmful actions!

If you drink responsibly, you could avoid the likelihood of fighting with friends due to your behaviour.

\section{DRINK RESPONSIBLY!}

\section{Internal guilt, male image}

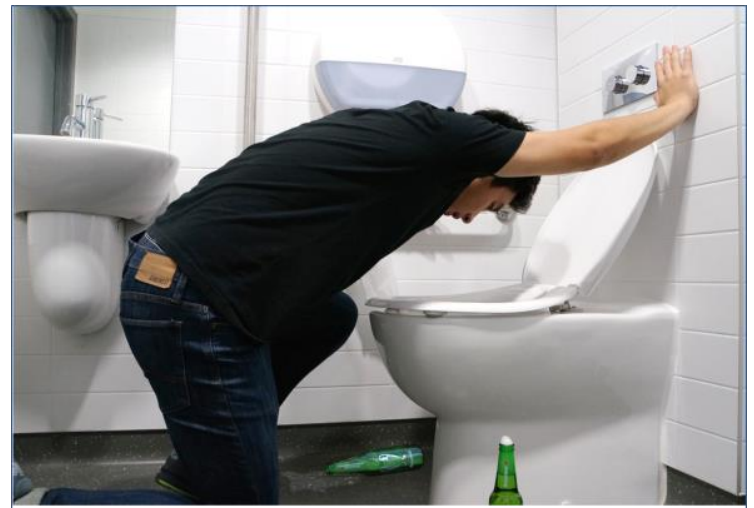

\section{GUILTY Moment of Life}

If you binge drink:

- You should feel GUILTY because YOU could suffer due to your irresponsible drinking behaviour.

- YOU could suffer the next day with a hangover so you could not do anything.

If you drink responsibly, you reduce the risk of being involved in a traffic accident at your own hand.

\section{DRINK RESPONSIBLY!}

2. Other-referenced guilt, male image

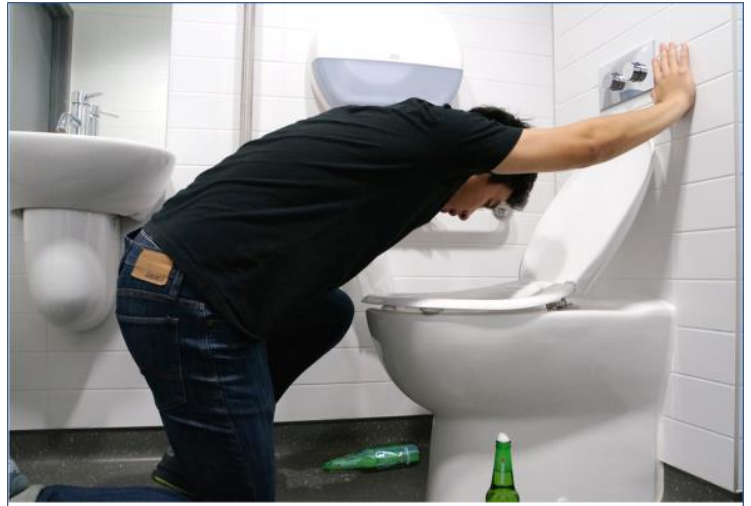

GUILTY Moment of Life

If PEOPLE binge drink:

- They should feel GUILTY because of their irresponsible drinking behaviour.

- They could get arrested due to their harmful actions!

If they drink responsibly, they could avoid the likelihood of fighting with friends due to their behaviour.

\section{DRINK RESPONSIBLY!}

\section{External guilt, male image}

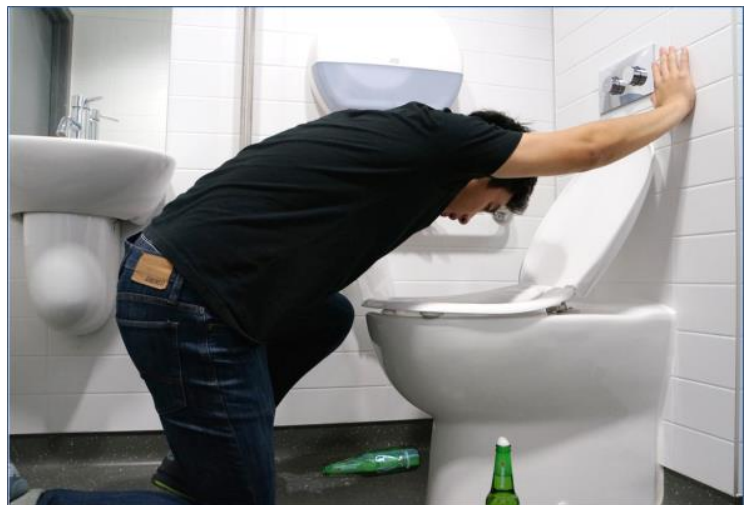

\section{GUILTY Moment of Life}

If you binge drink:

- You should feel GUILTY because your LOVED ONES could suffer due to your irresponsible drinking behaviour.

- Your FRIENDS could suffer the next day take care of your hangover.

If you drink responsibly, you reduce the risk of your FAMILY being injured in a traffic accident at your own hand.

\section{DRINK RESPONSIBLY!}




\section{Appendix 1. (Continued)}

5. Self-referenced shame, female image

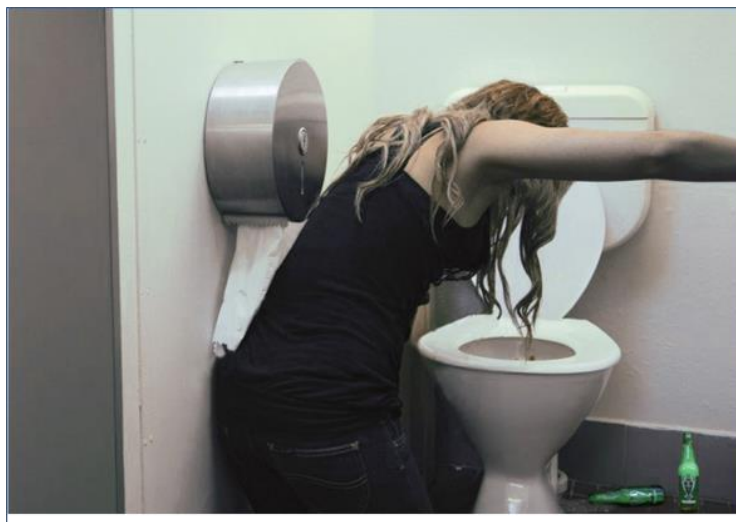

SHAMEFUL Moment of Life

If YOU are a binge drinker:

- You ought to be ASHAMED of yourself because you drink irresponsibly.

- You won't know where you are when you wake up - may be in jail or hospital?

If you drink irresponsibly, you create a bad image of yourself in the crowd.

\section{DRINK RESPONSIBLY!}

\section{Internal shame, female image}

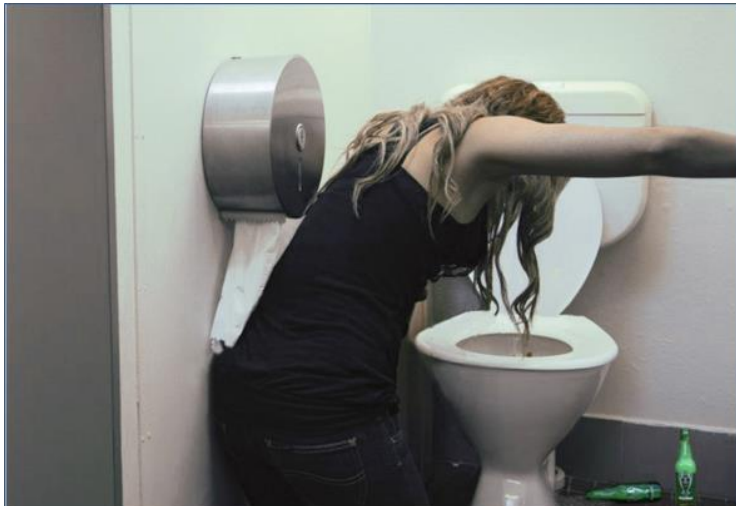

SHAMEFUL Moment of Life

If you are a binge drinker:

- You ought to be ASHAMED of yourself because you drink irresponsibly.

- YOU may face arrest for harm you caused!

If you drink irresponsibly, YOU could find yourself injured in a traffic accident.

\section{DRINK RESPONSIBLY!}

\section{Other-referenced shame, female image}

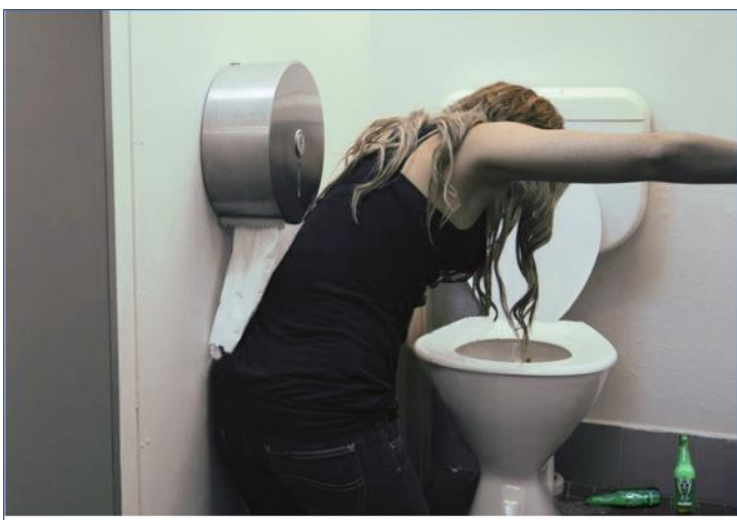

SHAMEFUL Moment of Life

If PEOPLE are binge drinkers:

- They ought to be ASHAMED of themselves because they drink irresponsibly.

- They won't know where they are when they wake up - may be in jail or hospital?

If they drink irresponsibly, they create a bad image of themselves in the crowd.

\section{DRINK RESPONSIBLY}

\section{External shame, female image}

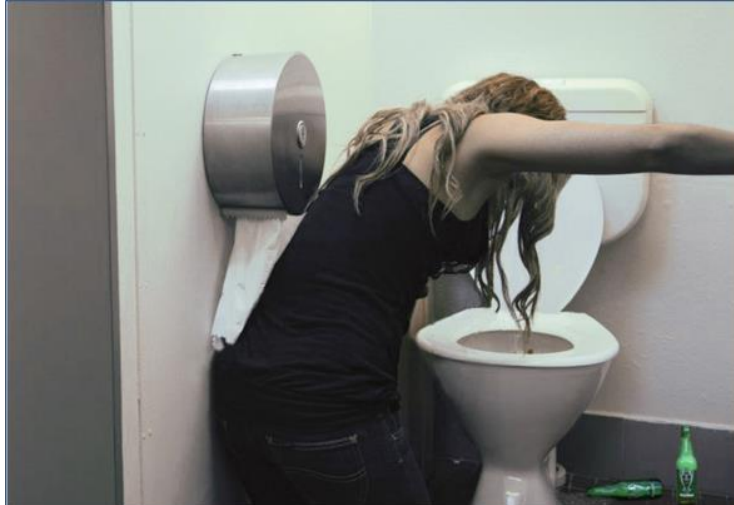

SHAMEFUL Moment of Life

If you are a binge drinker:

- You ought to be ASHAMED of yourself because your LOVED ONES could think of you as an irresponsible drinker.

- Your FRIENDS could find out you were arrested due to harm you caused.

If you drink irresponsibly, your FAMILY could find out you were in a traffic accident.

\section{DRINK RESPONSIBLY}




\section{Appendix 2. Order of Variables}

\section{MAIN SURVEY}

- Information sheet

- Instructions

- Stimulus presentation

- Part A - Health Communications

- Section 1. Manipulation check measures

- Emotional appeal (guilt-shame)

- Level of emotional appeal

- Self-referencing

- Source of evaluation

- Section 2. Mediator measures

- Emotional arousal

- Coping response

- Section 3. Dependent measures

- Attitude towards the ad

- Attitude towards the message

- Message compliance

- Binge drinking intention

- Part B - You as a Person

- Section 1. Control variable measures

- Health message exposure

- Message involvement

- Health value

- Alcohol use disorder

- Section 2. Moderator measures

- Self-construal

- Regulatory focus

- Personal cultural orientation

- Part C - Demographics

- Nationality

- Ethnicity

- Gender

- Age

- Part D - Further dependent measure

- Actual behaviour 
Appendix 3. Questionnaire

TE WHARE WĀNANGa O TE ŨPOKO O TE IKA A MÁUI

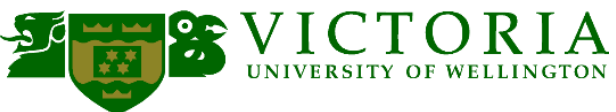

Health Communications Research

PARTICIPANT INFORMATION SHEET

Thank you for showing an interest in my research. This survey is being undertaken as part of my main study towards PhD at Victoria University of Wellington. The purpose of this study is to examine the effectiveness of health communications regarding binge drinking prevention. Victoria University of Wellington ethics approval has been obtained for this research (Ref: 21449).

This survey involves some discussion around lifestyle (binge drinking) and consequences. There is a possibility that some of the questions may make you feel uncomfortable. If you find this, you can choose not to answer certain questions, or you can choose to stop participating in the survey. If a problem arises and you wish, you can contact the Student Counselling, Mauri Ora, Level 1, Student Union Building, phone 044635310.

The survey is a chance for you to reflect on yourself and your lifestyle and hopefully will improve understanding of social marketers and public health administrators to motivate or prevent people from undertaking binge drinking.

Participation in this survey is completely voluntary. The survey will take around 15 to 20 minutes to complete. It can be terminated at any time and returned to at another time without losing previous responses. This is a secure website and all responses collected will remain anonymous. All printed information will be kept in a locked file with access restricted to the researcher. All electronic data will be kept in a password protected file only accessible by the researcher. Data collected in this survey will be destroyed after three years.

A thesis will be submitted to the School of Marketing and International Business and a final copy will be deposited in the University Library. Papers from this research project may also be submitted for academic conferences/publications.

Once you have completed this survey you may enter a draw to win one of two $\$ 100$ New World vouchers.

By completing this survey you consent to participate. If you have any questions or require any further information please contact myself or one of the study's supervisors:

\section{Main researcher:}

Nguyen Hoang Sinh

PhD Candidate

School of Marketing and International Business

Victoria University of Wellington

044638632

SinhHoang.Nguyen@vuw.ac.nz 
Supervisors:

Dr Daniel Laufer

Dr Jayne Krisjanous

Associate Professor

Senior Lecturer

School of Marketing and International Business

School of Marketing and International Business

Victoria University of Wellington

044635152

Victoria University of Wellington

044636023

Dan.Laufer@vuw.ac.nz

Jayne.Krisjanous@vuw.ac.nz 
Thank you for agreeing to complete this survey.

Are you an undergraduate student?

a. Yes

b. No [Participant will be directed to the following message before ending the survey]

Thank you for your time and taking interest in the study.

Unfortunately our survey requires undergraduate participants.

We are very sorry for the inconvenience.

What is your gender?

a. Male 1

b. Female 2

[Participant will be exposed to a male or female image advertisement accordingly]

Please read the instructions before you start the survey.

For Part A, you will be given a health advertisement regarding binge drinking. Please read the statement and answer your questions after viewing the advertisement.

In this part, binge drinking is defined as consuming 6 or more standard drinks (for men), or 4 or more standard drinks (for women) on a single occasion.

Next, please view the advertisement.

[Participant will be randomly given one of the eight advertisements] 


\section{PART A - Health Communications}

\section{Section 1}

Kindly answer your questions below based on your impression of the advertisement shown.

[To review the advertisement click on the "Previous" button at the bottom of the page.]

1. According to the advertisement, what was the focus of binge drinking?

The behaviour

The self
1
2
3
4
5

2. How successful was the advertisement in attempting to make the viewer feel quilt?

Not at All

Extremely 1

2

3

4

5

6

3. How successful was the advertisement in attempting to make the viewer feel shame?

Not at All

Extremely 1 2 3

4 5 6 7

4a. According to the advertisement, rate the extent to which binge drinking could be committed by...

\section{Yourself}

Others

1

$2 \quad 3$

4

5

6

4b. According to the advertisement, rate the extent to which the consequences of binge drinking could be known or seen by...

Yourself

1

2

3

4

5

Others

\section{Section 2}

Below are questions regarding your responses towards the advertisement. Please select 1 to 7 which you consider the most appropriate.

1. Please indicate the extent to which the following feelings describe your emotional arousal.

\begin{tabular}{|l|c|c|c|c|c|c|c|}
\hline & Not at All & \multicolumn{3}{c|}{ Somewhat } & \multicolumn{3}{c|}{ Extremely } \\
\hline a. bashful & 1 & 2 & 3 & 4 & 5 & 6 & 7 \\
\hline b. mortified & 1 & 2 & 3 & 4 & 5 & 6 & 7 \\
\hline c. shy & 1 & 2 & 3 & 4 & 5 & 6 & 7 \\
\hline d. humiliated & 1 & 2 & 3 & 4 & 5 & 6 & 7 \\
\hline e. abashed & 1 & 2 & 3 & 4 & 5 & 6 & 7 \\
\hline f. embarrassed & 1 & 2 & 3 & 4 & 5 & 6 & 7 \\
\hline g. depressed & 1 & 2 & 3 & 4 & 5 & 6 & 7 \\
\hline h. chided & 1 & 2 & 3 & 4 & 5 & 6 & 7 \\
\hline
\end{tabular}




\begin{tabular}{|l|c|c|c|c|c|c|c|}
\hline & Not at All & \multicolumn{3}{|c|}{ Somewhat } & \multicolumn{3}{c|}{ Extremely } \\
\hline i. reproached & 1 & 2 & 3 & 4 & 5 & 6 & 7 \\
\hline j. ashamed & 1 & 2 & 3 & 4 & 5 & 6 & 7 \\
\hline k. condemned & 1 & 2 & 3 & 4 & 5 & 6 & 7 \\
\hline I. unethical & 1 & 2 & 3 & 4 & 5 & 6 & 7 \\
\hline m. immoral & 1 & 2 & 3 & 4 & 5 & 6 & 7 \\
\hline n. delinquent & 1 & 2 & 3 & 4 & 5 & 6 & 7 \\
\hline o. unconscionable & 1 & 2 & 3 & 4 & 5 & 6 & 7 \\
\hline p. inappropriate & 1 & 2 & 3 & 4 & 5 & 6 & 7 \\
\hline q. wicked & 1 & 2 & 3 & 4 & 5 & 6 & 7 \\
\hline r. criminal & 1 & 2 & 3 & 4 & 5 & 6 & 7 \\
\hline s. liable & 1 & 2 & 3 & 4 & 5 & 6 & 7 \\
\hline t. indecent & 1 & 2 & 3 & 4 & 5 & 6 & 7 \\
\hline u. unscrupulous & 1 & 2 & 3 & 4 & 5 & 6 & 7 \\
\hline v. imprudent & 1 & 2 & 3 & 4 & 5 & 6 & 7 \\
\hline
\end{tabular}

2. Please feel free to describe the thoughts and feelings you experienced in response to the advertisement:

\section{$\underline{\text { Section } 3}$}

Below are questions regarding your attitudes and behavioural intentions towards the advertisement. Please select 1 to 7 which you consider the most appropriate.

1. Please rate your thoughts about the advertisement on these dimensions:

\begin{tabular}{|l|c|c|c|c|c|c|c|r|}
\hline uninformative & 1 & 2 & 3 & 4 & 5 & 6 & 7 & informative \\
\hline ineffective & 1 & 2 & 3 & 4 & 5 & 6 & 7 & effective \\
\hline not believable & 1 & 2 & 3 & 4 & 5 & 6 & 7 & believable \\
\hline
\end{tabular}

2. My feelings towards the message in the advertisement are that it is:

\begin{tabular}{|l|c|c|c|c|c|c|c|r|}
\hline not appropriate & 1 & 2 & 3 & 4 & 5 & 6 & 7 & appropriate \\
\hline harmful & 1 & 2 & 3 & 4 & 5 & 6 & 7 & beneficial \\
\hline unrealistic & 1 & 2 & 3 & 4 & 5 & 6 & 7 & realistic \\
\hline unconvincing & 1 & 2 & 3 & 4 & 5 & 6 & 7 & convincing \\
\hline
\end{tabular}


3. Please read each statement below and select your response according to how much you agree or disagree with each statement.

\begin{tabular}{|c|c|c|c|c|c|c|c|}
\hline \multirow[b]{2}{*}{$\begin{array}{l}\text { a. I intend to behave in ways that are } \\
\text { consistent with the advertisement. }\end{array}$} & \multicolumn{3}{|c|}{$\begin{array}{l}\text { Strongly } \\
\text { Disagree }\end{array}$} & \multicolumn{3}{|c|}{ Neutral } & \multirow{2}{*}{$\begin{array}{c}\text { Strongly } \\
\text { Agree }\end{array}$} \\
\hline & 1 & 2 & 3 & 4 & 5 & 6 & \\
\hline $\begin{array}{l}\text { b. I am going to make an effort to do what } \\
\text { the advertisement urged me to do. }\end{array}$ & 1 & 2 & 3 & 4 & 5 & 6 & 7 \\
\hline $\begin{array}{l}\text { c. I plan to act in ways that are compatible } \\
\text { with the position promoted by the } \\
\text { advertisement. }\end{array}$ & 1 & 2 & 3 & 4 & 5 & 6 & 7 \\
\hline
\end{tabular}

4. How likely are you to engage in binge drinking this year?

Binge drinking is defined as consuming 6 or more standard drinks (for men), or 4 or more standard drinks (for women) on a single occasion. A 330ml (small) can of beer is approx. 1 standard drink, a $100 \mathrm{ml}$ (small) glass of wine is approx. 1 standard drink.

Not at all likely

1

2

3

4

5

Very likely

2

\section{PART B - You as a Person}

\section{Section 1}

All questions in Section 1 concern health issues in general. Please select the most appropriate option.

1. How much attention, if any, have you paid to health communications you have heard or seen in the last three months?
a. None
1
b. Very little
2
c. Some
3
d. A lot
4 
2. To me, health issues

\begin{tabular}{|l|c|c|c|c|c|c|c|r|}
\hline are unimportant & 1 & 2 & 3 & 4 & 5 & 6 & 7 & are important \\
\hline are of no concern & 1 & 2 & 3 & 4 & 5 & 6 & 7 & are of concern \\
\hline are irrelevant & 1 & 2 & 3 & 4 & 5 & 6 & 7 & are relevant \\
\hline mean nothing & 1 & 2 & 3 & 4 & 5 & 6 & 7 & mean a lot \\
\hline do not matter & 1 & 2 & 3 & 4 & 5 & 6 & 7 & matter \\
are insignificant & 1 & 2 & 3 & 4 & 5 & 6 & 7 & are significant \\
\hline
\end{tabular}

3. Please read each statement below and select your response according to how much you agree or disagree with each statement.

\begin{tabular}{|c|c|c|c|c|c|c|c|}
\hline \multirow[b]{2}{*}{$\begin{array}{l}\text { a. If you don't have your health, you don't } \\
\text { have anything. }\end{array}$} & \multicolumn{3}{|c|}{$\begin{array}{l}\text { Strongly } \\
\text { Disagree }\end{array}$} & \multicolumn{3}{|c|}{ Neutral } & \multirow{2}{*}{$\begin{array}{r}\begin{array}{r}\text { Strongly } \\
\text { Agree }\end{array} \\
7\end{array}$} \\
\hline & 1 & 2 & 3 & 4 & 5 & 6 & \\
\hline $\begin{array}{l}\text { b. There are many things I care about more } \\
\text { than my health. }\end{array}$ & 1 & 2 & 3 & 4 & 5 & 6 & 7 \\
\hline $\begin{array}{l}\text { c. Good health is of only minor importance in } \\
\text { a happy life. }\end{array}$ & 1 & 2 & 3 & 4 & 5 & 6 & 7 \\
\hline $\begin{array}{l}\text { d. There is nothing more important than good } \\
\text { health. }\end{array}$ & 1 & 2 & 3 & 4 & 5 & 6 & 7 \\
\hline
\end{tabular}

4. Please read each question below and select your answer that is correct for you.

Remember that a $330 \mathrm{ml}$ (small) can of beer is approx. 1 standard drink, a $100 \mathrm{ml}$ (small) glass of wine is approx. 1 standard drink.

\begin{tabular}{|l|c|c|c|c|c|}
\hline & Never & $\begin{array}{c}\text { Monthly or } \\
\text { less }\end{array}$ & $\begin{array}{c}2-4 \\
\text { times/month }\end{array}$ & $\begin{array}{c}3-4 \\
\text { times/week }\end{array}$ & $\begin{array}{c}4+ \\
\text { times/week }\end{array}$ \\
\hline $\begin{array}{l}\text { a. How often do you have a drink } \\
\text { containing alcohol? }\end{array}$ & $\begin{array}{c}0 \\
{[\text { skip to Qs }} \\
\text { 4.i \& 4.J] }\end{array}$ & 1 & 2 & 3 & 4 \\
\hline
\end{tabular}

\begin{tabular}{|l|c|c|c|c|c|}
\hline & 1 or 2 & 3 or 4 & 5 or 6 & 7 to 9 & 10 or more \\
\hline $\begin{array}{l}\text { b. How many standard drinks do you } \\
\text { have on a typical day when you are } \\
\text { drinking? }\end{array}$ & 0 & 1 & 2 & 3 & 4 \\
\hline
\end{tabular}




\begin{tabular}{|l|c|c|c|c|c|}
\hline & Never & $\begin{array}{c}\text { Less than } \\
\text { monthly }\end{array}$ & Monthly & Weekly & $\begin{array}{c}\text { Daily or } \\
\text { almost daily }\end{array}$ \\
\hline $\begin{array}{l}\text { c. How often do you have six or more } \\
\text { standard drinks on one occasion? }\end{array}$ & 0 & 1 & 2 & 3 & 4 \\
\hline $\begin{array}{l}\text { d. How often during the last year have } \\
\text { you found that you were not able to } \\
\text { stop drinking once you had started? }\end{array}$ & 0 & 1 & 2 & 3 & 4 \\
\hline $\begin{array}{l}\text { e. How often during the last year have } \\
\text { you failed to do what was normally } \\
\text { expected of you because of drinking? }\end{array}$ & 0 & 1 & 2 & 3 & 4 \\
\hline $\begin{array}{l}\text { f. How often during the last year have } \\
\text { you needed a first drink in the morning } \\
\text { to get yourself going after a heavy } \\
\text { drinking session? }\end{array}$ & 0 & 1 & 2 & 3 & 4 \\
\hline $\begin{array}{l}\text { g. How often during the last year have } \\
\text { you had a feeling of guilt or remorse } \\
\text { after drinking? }\end{array}$ & 0 & 1 & 2 & 3 & 3 \\
\hline $\begin{array}{l}\text { h. How often during the last year have } \\
\text { you been unable to remember what } \\
\text { happened the night before because } \\
\text { you had been drinking? }\end{array}$ & 0 & 1 & & 3 & 4 \\
\hline
\end{tabular}

\begin{tabular}{|l|c|c|c|}
\hline & No & $\begin{array}{c}\text { Yes, but not in the last } \\
\text { year }\end{array}$ & $\begin{array}{c}\text { Yes, during the last } \\
\text { year }\end{array}$ \\
\hline $\begin{array}{l}\text { i. Have you or someone else been } \\
\text { injured because of your drinking? }\end{array}$ & 0 & 2 & 4 \\
\hline $\begin{array}{l}\text { j. Has a relative, friend, doctor or } \\
\text { other health care worker been } \\
\text { concerned about your drinking or } \\
\text { suggested you cut down? }\end{array}$ & 0 & 2 & 4 \\
\hline
\end{tabular}

\section{Section 2}

Respond to all questions in Section 2 according to how you would normally feel. Please select 1 to 7 which you consider the most appropriate.

1. Please read each statement below and select your response according to how much you agree or disagree with each statement. 


\begin{tabular}{|c|c|c|c|c|c|c|c|}
\hline \multirow[b]{2}{*}{$\begin{array}{l}\text { a. I have respect for the authority figures with } \\
\text { whom I interact. }\end{array}$} & \multicolumn{3}{|c|}{$\begin{array}{l}\text { Strongly } \\
\text { Disagree }\end{array}$} & \multicolumn{3}{|c|}{ Neutral } & \multirow{2}{*}{$\begin{array}{r}\begin{array}{r}\text { Strongly } \\
\text { Agree }\end{array} \\
7\end{array}$} \\
\hline & 1 & 2 & 3 & 4 & 5 & 6 & \\
\hline $\begin{array}{l}\text { b. I will sacrifice my self-interests for the } \\
\text { benefit of the group I am in. }\end{array}$ & 1 & 2 & 3 & 4 & 5 & 6 & 7 \\
\hline $\begin{array}{l}\text { c. My happiness depends on the happiness } \\
\text { of those in my group. }\end{array}$ & 1 & 2 & 3 & 4 & 5 & 6 & 7 \\
\hline $\begin{array}{l}\text { d. I would offer my seat on the bus to my } \\
\text { professor. }\end{array}$ & 1 & 2 & 3 & 4 & 5 & 6 & 7 \\
\hline $\begin{array}{l}\text { e. I respect people who are modest about } \\
\text { themselves. }\end{array}$ & 1 & 2 & 3 & 4 & 5 & 6 & 7 \\
\hline $\begin{array}{l}\text { f. It is important for me to maintain harmony } \\
\text { within my group. }\end{array}$ & 1 & 2 & 3 & 4 & 5 & 6 & 7 \\
\hline $\begin{array}{l}\text { g. I often have the feeling that my } \\
\text { relationships with others are more important } \\
\text { than my own accomplishments. }\end{array}$ & 1 & 2 & 3 & 4 & 5 & 6 & 7 \\
\hline $\begin{array}{l}\text { h. I should take into consideration my } \\
\text { parents' advice when making } \\
\text { education/career plans. }\end{array}$ & 1 & 2 & 3 & 4 & 5 & 6 & 7 \\
\hline $\begin{array}{l}\text { i. It is important to me to respect decisions } \\
\text { made by my group. }\end{array}$ & 1 & 2 & 3 & 4 & 5 & 6 & 7 \\
\hline $\begin{array}{l}\text { j. I will stay in a group if they need me, even } \\
\text { when I'm not happy with the group. }\end{array}$ & 1 & 2 & 3 & 4 & 5 & 6 & 7 \\
\hline $\begin{array}{l}\text { k. If my brother or sister fails, I feel } \\
\text { responsible. }\end{array}$ & 1 & 2 & 3 & 4 & 5 & 6 & 7 \\
\hline $\begin{array}{l}\text { I. Even when I strongly disagree with group } \\
\text { members, I avoid an argument. }\end{array}$ & 1 & 2 & 3 & 4 & 5 & 6 & 7 \\
\hline $\begin{array}{l}\text { m. l'd rather say "No" directly than risk being } \\
\text { misunderstood. }\end{array}$ & 1 & 2 & 3 & 4 & 5 & 6 & 7 \\
\hline $\begin{array}{l}\text { n. Speaking up in a work/task group/class is } \\
\text { not a problem for me. }\end{array}$ & 1 & 2 & 3 & 4 & 5 & 6 & 7 \\
\hline $\begin{array}{l}\text { o. Having a lively imagination is important to } \\
\text { me. }\end{array}$ & 1 & 2 & 3 & 4 & 5 & 6 & 7 \\
\hline $\begin{array}{l}\text { p. I am comfortable being singled out for } \\
\text { praise or rewards. }\end{array}$ & 1 & 2 & 3 & 4 & 5 & 6 & 7 \\
\hline
\end{tabular}




\begin{tabular}{|l|c|c|c|c|c|c|c|}
\hline \multicolumn{3}{|l|}{$\begin{array}{l}\text { Strongly } \\
\text { Disagree }\end{array}$} & \multicolumn{5}{|c|}{$\begin{array}{r}\text { Strongly } \\
\text { Agree }\end{array}$} \\
\hline $\begin{array}{l}\text { q. I am the same person at home that I am } \\
\text { at school. }\end{array}$ & 1 & 2 & 3 & 4 & 5 & 6 & 7 \\
\hline $\begin{array}{l}\text { r. Being able to take care of myself is a } \\
\text { primary concern for me. }\end{array}$ & 1 & 2 & 3 & 4 & 5 & 6 & 7 \\
\hline $\begin{array}{l}\text { s. I act the same way no matter who I am with. } \\
\text { t. I feel comfortable using someone's first } \\
\text { name soon after I meet them, even when } \\
\text { they are much older than I am. }\end{array}$ & 1 & 2 & 3 & 4 & 5 & 6 & 7 \\
\hline $\begin{array}{l}\text { u. I prefer to be direct and forthright when } \\
\text { dealing with people I've just met. }\end{array}$ & 1 & 2 & 3 & 4 & 5 & 6 & 7 \\
\hline $\begin{array}{l}\text { v. I enjoy being unique and different from } \\
\text { others in many respects. }\end{array}$ & 1 & 2 & 3 & 4 & 5 & 6 & 7 \\
\hline $\begin{array}{l}\text { w. My personal identity, independent of } \\
\text { others, is very important to me. }\end{array}$ & 1 & 2 & 3 & 4 & 5 & 6 & 7 \\
\hline
\end{tabular}

2. Please read each statement below and select your response according to how true or untrue each statement is.

\begin{tabular}{|c|c|c|c|c|c|c|c|}
\hline & $\begin{array}{l}\text { Not a } \\
\text { True }\end{array}$ & & & $\mathrm{Neu}$ & & & $\begin{array}{r}\text { remely } \\
\text { True }\end{array}$ \\
\hline $\begin{array}{l}\text { a. In general, I am focused on achieving } \\
\text { positive outcomes in my life. }\end{array}$ & 1 & 2 & 3 & 4 & 5 & 6 & 7 \\
\hline $\begin{array}{l}\text { b. I frequently imagine how I will achieve my } \\
\text { hopes and aspirations. }\end{array}$ & 1 & 2 & 3 & 4 & 5 & 6 & 7 \\
\hline $\begin{array}{l}\text { c. I often imagine myself experiencing good } \\
\text { things that I hope will happen to me. }\end{array}$ & 1 & 2 & 3 & 4 & 5 & 6 & 7 \\
\hline $\begin{array}{l}\text { d. Overall, I am more oriented towards } \\
\text { achieving success than preventing failure. }\end{array}$ & 1 & 2 & 3 & 4 & 5 & 6 & 7 \\
\hline $\begin{array}{l}\text { e. I often think about the person I would } \\
\text { ideally like to be in the future. }\end{array}$ & 1 & 2 & 3 & 4 & 5 & 6 & 7 \\
\hline $\begin{array}{l}\text { f. I typically focus on the success I hope to } \\
\text { achieve in the future. }\end{array}$ & 1 & 2 & 3 & 4 & 5 & 6 & 7 \\
\hline $\begin{array}{l}\text { g. I often think about how I will achieve } \\
\text { academic success. }\end{array}$ & 1 & 2 & 3 & 4 & 5 & 6 & 7 \\
\hline $\begin{array}{l}\text { h. My major goal in university right now is to } \\
\text { achieve my academic ambitions. }\end{array}$ & 1 & 2 & 3 & 4 & 5 & 6 & 7 \\
\hline
\end{tabular}




\begin{tabular}{|c|c|c|c|c|c|c|c|}
\hline & $\begin{array}{l}\text { Not a } \\
\text { True }\end{array}$ & & & $\mathrm{Neu}$ & & & $\begin{array}{r}\text { remely } \\
\text { True }\end{array}$ \\
\hline $\begin{array}{l}\text { i. I see myself as someone who is primarily } \\
\text { striving to reach my "ideal self" - to fulfil my } \\
\text { hopes, wishes, and aspirations. }\end{array}$ & 1 & 2 & 3 & 4 & 5 & 6 & 7 \\
\hline $\begin{array}{l}\text { j. In general, I am focused on preventing } \\
\text { negative events in my life. }\end{array}$ & 1 & 2 & 3 & 4 & 5 & 6 & 7 \\
\hline $\begin{array}{l}\text { k. I am anxious that I will fall short of my } \\
\text { responsibilities and obligations. }\end{array}$ & 1 & 2 & 3 & 4 & 5 & 6 & 7 \\
\hline $\begin{array}{l}\text { I. I often imagine myself experiencing bad } \\
\text { things that I fear might happen to me. }\end{array}$ & 1 & 2 & 3 & 4 & 5 & 6 & 7 \\
\hline $\begin{array}{l}\text { m. I am more oriented towards preventing } \\
\text { losses than I am towards achieving gains. }\end{array}$ & 1 & 2 & 3 & 4 & 5 & 6 & 7 \\
\hline $\begin{array}{l}\text { n. I often think about the person I am afraid I } \\
\text { might become in the future. }\end{array}$ & 1 & 2 & 3 & 4 & 5 & 6 & 7 \\
\hline $\begin{array}{l}\text { o. I frequently think about how I can prevent } \\
\text { failures in my life. }\end{array}$ & 1 & 2 & 3 & 4 & 5 & 6 & 7 \\
\hline $\begin{array}{l}\text { p. I often worry that I will fail to accomplish } \\
\text { my academic goals. }\end{array}$ & 1 & 2 & 3 & 4 & 5 & 6 & 7 \\
\hline $\begin{array}{l}\text { q. My major goal in university right now is to } \\
\text { avoid becoming an academic failure. }\end{array}$ & 1 & 2 & 3 & 4 & 5 & 6 & 7 \\
\hline $\begin{array}{l}\text { r. I see myself as someone who is primarily } \\
\text { striving to become the self I "ought" to be - to } \\
\text { fulfil my duties, responsibilities, and } \\
\text { obligations. }\end{array}$ & 1 & 2 & 3 & 4 & 5 & 6 & 7 \\
\hline
\end{tabular}

3. Please read each statement below and select your response according to how much you agree or disagree with each statement.

\begin{tabular}{|c|c|c|c|c|c|c|c|}
\hline \multirow[b]{2}{*}{$\begin{array}{l}\text { a. Individuals should sacrifice self-interest for } \\
\text { the group that they belong to. }\end{array}$} & \multicolumn{3}{|c|}{$\begin{array}{l}\text { Strongly } \\
\text { Disagree }\end{array}$} & \multicolumn{3}{|c|}{ Neutral } & \multirow{2}{*}{$\begin{array}{r}\begin{array}{r}\text { Strongly } \\
\text { Agree }\end{array} \\
7\end{array}$} \\
\hline & 1 & 2 & 3 & 4 & 5 & 6 & \\
\hline $\begin{array}{l}\text { b. Individuals should stick with the group } \\
\text { even through difficulties. }\end{array}$ & 1 & 2 & 3 & 4 & 5 & 6 & 7 \\
\hline $\begin{array}{l}\text { c. Group welfare is more important than } \\
\text { individual rewards. }\end{array}$ & 1 & 2 & 3 & 4 & 5 & 6 & 7 \\
\hline
\end{tabular}




\begin{tabular}{|l|c|c|c|c|c|c|c|}
\hline & \multicolumn{2}{|l|}{$\begin{array}{l}\text { Strongly } \\
\text { Disagree }\end{array}$} & \multicolumn{2}{|l|}{$\begin{array}{r}\text { Agrongly } \\
\text { Agree }\end{array}$} \\
\hline $\begin{array}{l}\text { d. Group success is more important than } \\
\text { individual success. }\end{array}$ & 1 & 2 & 3 & 4 & 5 & 6 & 7 \\
\hline $\begin{array}{l}\text { e. Individuals should pursue their goals after } \\
\text { considering the welfare of the group. }\end{array}$ & 1 & 2 & 3 & 4 & 5 & 6 & 7 \\
\hline $\begin{array}{l}\text { f. Group loyalty should be encouraged even } \\
\text { if individual goals suffer. }\end{array}$ & 1 & 2 & 3 & 4 & 5 & 6 & 7 \\
\hline
\end{tabular}

\section{PART C - About Yourself}

1. Please indicate your nationality.

a. New Zealand

b. Other (please specify) [skip to Q4]

2. Have you lived in your country since birth?

a. Yes 1

b. No 2

3. Which is the main ethnic group you belong to?
a. European/Pākehā
1
b. Māori
2
c. Asian
3
d. Pacific peoples
4
e. Middle Eastern
f. Latin American
6
g. African
7
h. Other (please specify)

4. Please indicate your age.

(in years) 


\section{PART D - Further Information}

If you are interested in learning more about responsible drinking, click on this hyperlink to go to the Health Promotion Agency site offering information, advice and resources for responsible drinking.

1. Did you click on the hyperlink?

a. Yes 1

b. No 2 [skip to prize draw]

2. Did you look at the site that provided additional information regarding responsible drinking?

a. Yes 1

b. No 2

Thank you for your participation. It is very much appreciated.

\section{Prize Draw}

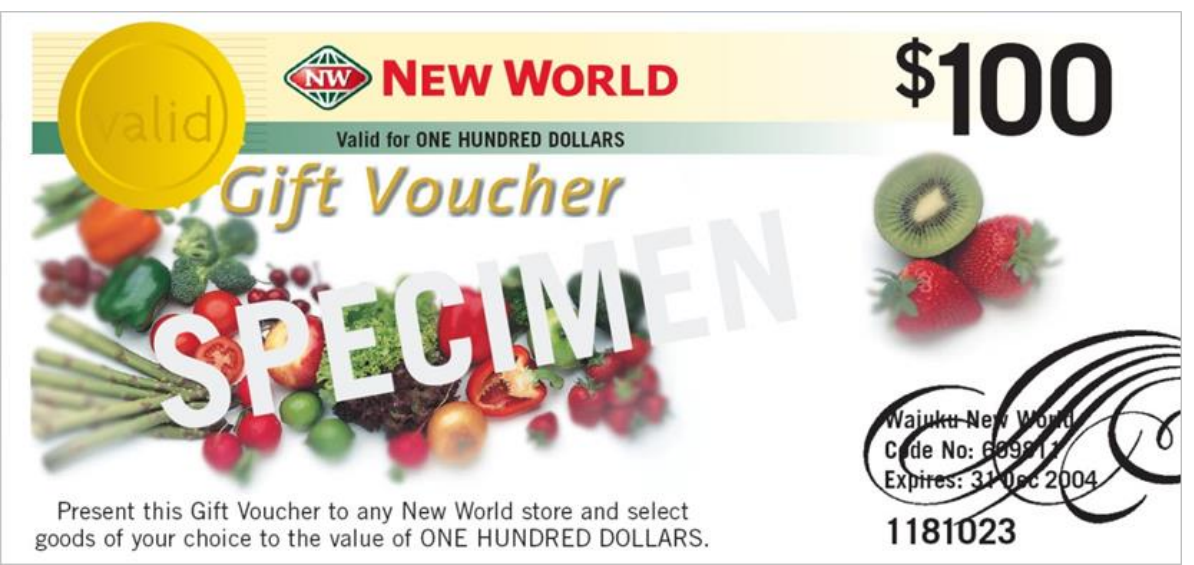

If you wish to be entered in the draw for one of two $\$ 100$ New World vouchers, please include your email address.

Email:

Please be assured that your anonymity from answering the survey is still retained. 


\section{Appendix 4. Measurements of Constructs}

\begin{tabular}{|c|c|c|c|c|c|}
\hline Constructs & Dimensions & Items & Codes & Anchors & Sources \\
\hline \multirow{2}{*}{$\begin{array}{l}\text { Emotional } \\
\text { appeal (EAp) }\end{array}$} & $\begin{array}{l}\text { Guilt appeal } \\
\text { (GAp) }\end{array}$ & $\begin{array}{l}\text { 1. How successful was the advertisement in attempting to make the viewer } \\
\text { feel guilt? }\end{array}$ & GAp1 & \multirow{2}{*}{$\begin{array}{l}\text { 7-point scale: } \\
\text { 1='not at all true' } \\
\text { 7='extremely true' }\end{array}$} & \multirow{2}{*}{$\begin{array}{l}\text { Adapted from } \\
\text { Coulter and } \\
\text { Pinto (1995) }\end{array}$} \\
\hline & $\begin{array}{l}\text { Shame appeal } \\
\text { (SAp) }\end{array}$ & $\begin{array}{l}\text { 2. How successful was the advertisement in attempting to make the viewer } \\
\text { feel shame? }\end{array}$ & SAp1 & & \\
\hline \multirow{17}{*}{$\begin{array}{l}\text { Emotional } \\
\text { arousal (EAr) }\end{array}$} & \multirow{12}{*}{$\begin{array}{l}\text { Guilt arousal } \\
\text { (GAr) }\end{array}$} & 1. condemned; & GAr1 & 7-point scale: & Adopted from \\
\hline & & 2. unethical; & GAr2 & 1='not at all' & Hoblitzelle \\
\hline & & 3. immoral; & GAr3 & 7='extremely' & $(1987): \alpha=.86$ \\
\hline & & 4. delinquent; & GAr4 & & for shame and \\
\hline & & 5. unconscionable; & GAr5 & & .88 for guilt \\
\hline & & 6. inappropriate; & GAr6 & & \\
\hline & & 7. wicked; & GAr7 & & \\
\hline & & 8. criminal; & GAr8 & & \\
\hline & & 9. liable; & GAr9 & & \\
\hline & & 10. indecent; & GAr10 & & \\
\hline & & 11. unscrupulous; & GAr11 & & \\
\hline & & 12. imprudent & GAr12 & & \\
\hline & \multirow{5}{*}{$\begin{array}{l}\text { Shame arousal } \\
(\mathrm{SAr})\end{array}$} & 1. bashful; & SAr1 & & \\
\hline & & 2. mortified; & SAr2 & & \\
\hline & & 3. shy; & SAr3 & & \\
\hline & & 4. humiliated; & SAr4 & & \\
\hline & & 5. abashed; & SAr5 & & \\
\hline
\end{tabular}




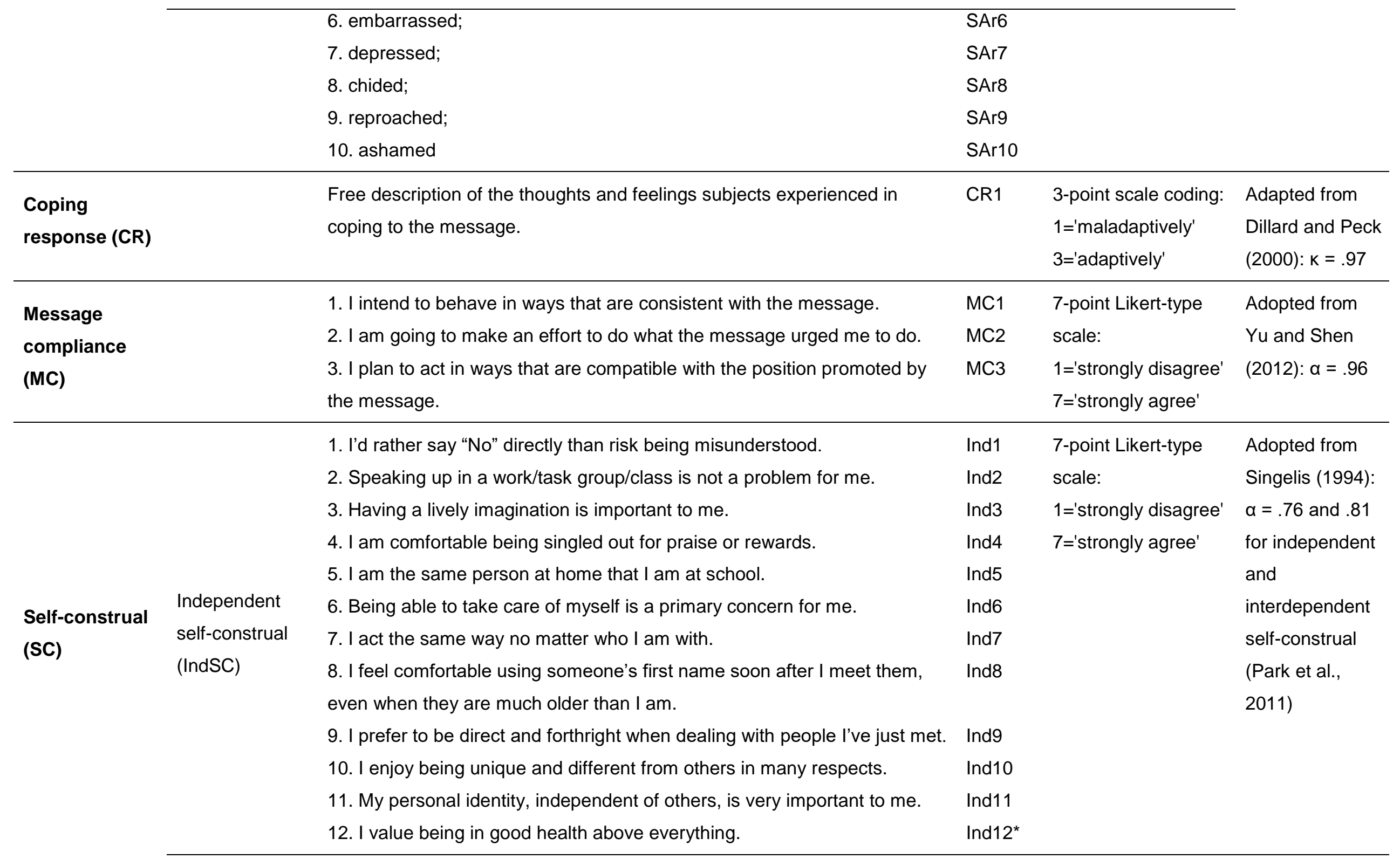




\begin{tabular}{|c|c|c|c|c|c|}
\hline & \multirow{12}{*}{$\begin{array}{l}\text { Interdependent } \\
\text { self-construal } \\
\text { (IntSC) }\end{array}$} & 1. I have respect for the authority figures with whom I interact. & Int1 & & \\
\hline & & 2. It is important for me to maintain harmony within my group. & Int2 & & \\
\hline & & 3. My happiness depends on the happiness of those in my group. & Int3 & & \\
\hline & & 4. I would offer my seat on the bus to my professor. & $\ln 4$ & & \\
\hline & & 5. I respect people who are modest about themselves. & Int5 & & \\
\hline & & 6. I will sacrifice my self-interests for the benefit of the group I am in. & Int6 & & \\
\hline & & $\begin{array}{l}\text { 7. I often have the feeling that my relationships with others are more } \\
\text { important than my own accomplishments. }\end{array}$ & Int7 & & \\
\hline & & $\begin{array}{l}\text { 8. I should take into consideration my parents' advice when making } \\
\text { education/career plans. }\end{array}$ & Int8 & & \\
\hline & & 9. It is important to me to respect decisions made by my group. & Int9 & & \\
\hline & & $\begin{array}{l}\text { 10. I will stay in a group if they need me, even when I'm not happy with the } \\
\text { group. }\end{array}$ & $\ln 10$ & & \\
\hline & & 11. If my brother or sister fails, I feel responsible. & Int11 & & \\
\hline & & $\begin{array}{l}\text { 12. Even when I strongly disagree with group members, I avoid an } \\
\text { argument. }\end{array}$ & Int12 & & \\
\hline \multirow{7}{*}{$\begin{array}{l}\text { Regulatory } \\
\text { focus (RF) }\end{array}$} & \multirow{7}{*}{$\begin{array}{l}\text { Promotion } \\
\text { focus (ProRF) }\end{array}$} & 1. In general, I am focused on achieving positive outcomes in my life. & Pro1 & 7-point scale: & Adopted from \\
\hline & & 2. I frequently imagine how I will achieve my hopes and aspirations. & Pro2 & $1=$ 'not at all true' & Lockwood et al. \\
\hline & & $\begin{array}{l}\text { 3. I often imagine myself experiencing good things that I hope will happen } \\
\text { to me. }\end{array}$ & Pro3 & $7=$ 'extremely true' & $\begin{array}{l}(2002): \alpha=.87 \\
\text { for promotion }\end{array}$ \\
\hline & & $\begin{array}{l}\text { 4. Overall, I am more oriented towards achieving success than preventing } \\
\text { failure. }\end{array}$ & Pro4 & & $\begin{array}{l}\text { and } .82 \text { for } \\
\text { prevention focus }\end{array}$ \\
\hline & & 5. I often think about the person I would ideally like to be in the future. & Pro5 & & (Zhao \& \\
\hline & & 6. I typically focus on the success I hope to achieve in the future. & Pro6 & & Pechmann, \\
\hline & & 7. I often think about how I will achieve academic success. & Pro7 & & 2007) \\
\hline
\end{tabular}




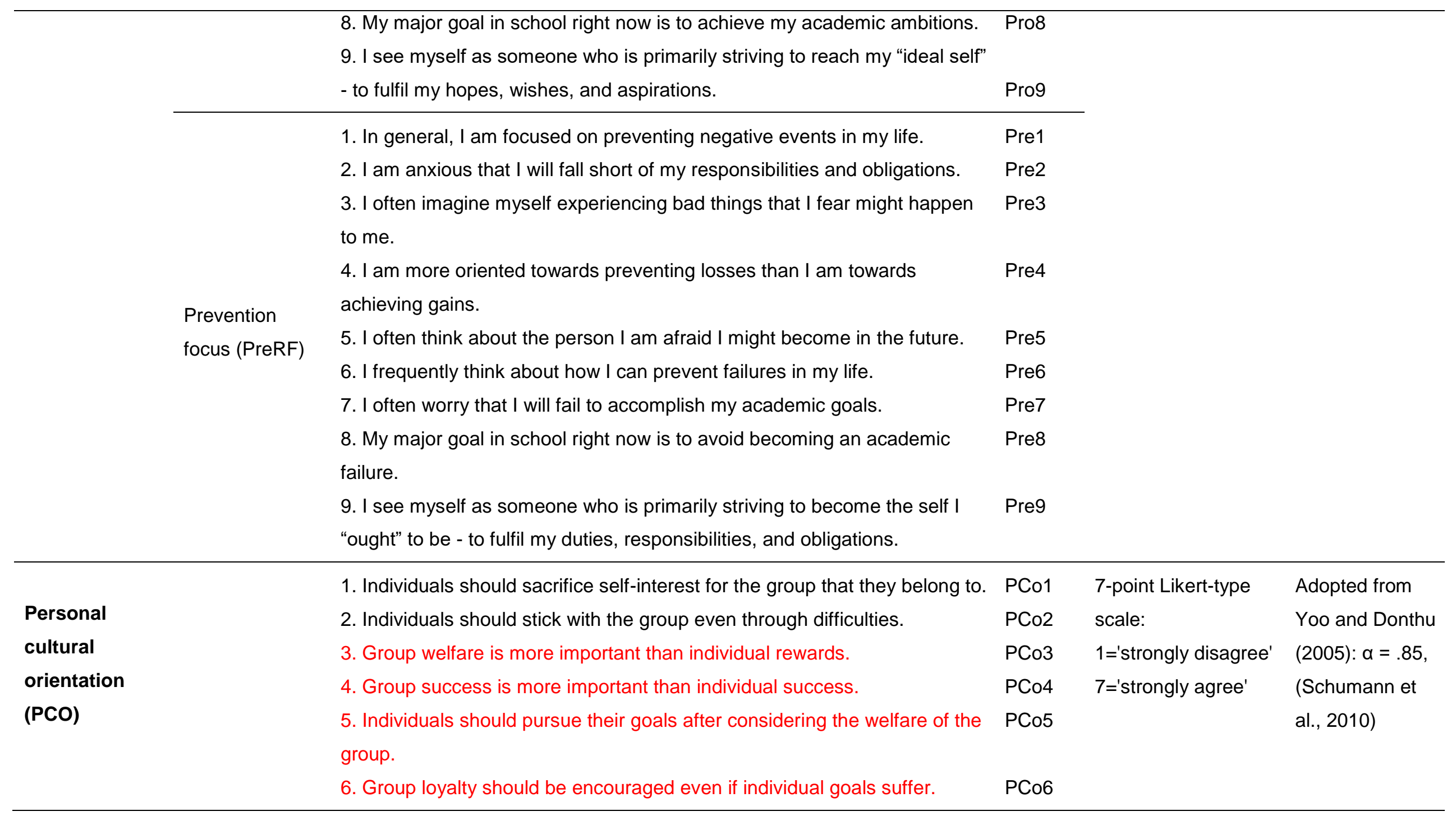

* This item was removed due to redundancy with item 4 of the health value construct (see Questionnaire in Appendix 3). 


\section{Appendix 5. Normality Test}

\begin{tabular}{|c|c|c|c|c|c|}
\hline Items & Skewness & Kurtosis & Items & Skewness & Kurtosis \\
\hline GAp1 & .026 & -1.055 & \multicolumn{3}{|c|}{ Independent self-construal (IndSC) } \\
\hline SAp1 & -.223 & -1.087 & Ind1 & -.429 & -.568 \\
\hline \multicolumn{3}{|c|}{ Guilt arousal (GAr) } & Ind2 & -.418 & -.811 \\
\hline GAr1 & .247 & -1.326 & Ind3 & -.650 & .410 \\
\hline GAr2 & .355 & -1.221 & Ind4 & -.510 & -.550 \\
\hline GAr3 & .372 & -1.198 & Ind5 & -.411 & -.844 \\
\hline GAr4 & .264 & -1.204 & Ind6 & -.807 & .491 \\
\hline GAr5 & .412 & -1.195 & Ind7 & -.134 & -1.076 \\
\hline GAr6 & .143 & -1.280 & Ind8 & -.540 & -.491 \\
\hline GAr7 & .714 & -.897 & Ind9 & -.223 & -.664 \\
\hline GAr8 & .798 & -.782 & Ind10 & -.387 & -.062 \\
\hline GAr9 & .180 & -1.371 & Ind11 & -.749 & .341 \\
\hline GAr10 & .154 & -1.381 & Ind12 & -.244 & -.909 \\
\hline GAr11 & .534 & -.939 & \multicolumn{3}{|c|}{ Interdependent self-construal (IntSC) } \\
\hline GAr12 & .607 & -.823 & Int1 & -1.452 & 2.360 \\
\hline \multicolumn{3}{|c|}{ Shame arousal (SAr) } & Int2 & -.569 & -.228 \\
\hline SAr1 & .715 & -.576 & Int3 & -.243 & -.625 \\
\hline SAr2 & .527 & -.922 & Int4 & -.880 & .044 \\
\hline SAr3 & 1.136 & .270 & Int5 & -1.120 & 1.295 \\
\hline SAr4 & .489 & -1.157 & Int6 & -1.135 & 1.856 \\
\hline SAr5 & .657 & -.821 & Int7 & -.303 & -.656 \\
\hline SAr6 & .329 & -1.267 & Int8 & -.667 & .070 \\
\hline SAr7 & .876 & -.369 & Int9 & -.792 & .891 \\
\hline SAr8 & .457 & -1.143 & Int10 & -.213 & -.853 \\
\hline SAr9 & .524 & -1.001 & Int11 & .129 & -1.137 \\
\hline SAr10 & .402 & -1.202 & Int12 & .056 & -1.037 \\
\hline CR1 & -.095 & -1.856 & \multicolumn{3}{|c|}{ Promotion focus (ProRF) } \\
\hline \multicolumn{3}{|c|}{ Message compliance (MC) } & Pro1 & -1.290 & 2.001 \\
\hline MC1 & .309 & -.934 & Pro2 & -1.044 & 1.305 \\
\hline MC2 & .295 & -1.018 & Pro3 & -1.234 & 1.370 \\
\hline MC3 & .036 & -1.007 & Pro4 & -.628 & -.079 \\
\hline \multicolumn{3}{|c|}{ Personal cultural orientation (PCO) } & Pro5 & -1.075 & .861 \\
\hline PCo1 & .147 & -.910 & Pro6 & -.859 & .437 \\
\hline PCo2 & -.236 & -.650 & Pro7 & -.577 & -.164 \\
\hline PCo3 & -.140 & -.456 & Pro8 & -1.024 & 1.016 \\
\hline PCo4 & -.184 & -.535 & Pro9 & -1.095 & 1.179 \\
\hline PCo5 & -.248 & -.708 & \multicolumn{3}{|c|}{ Prevention focus (PreRF) } \\
\hline \multirow[t]{9}{*}{ PCo6 } & .058 & -.848 & Pre1 & -.714 & .275 \\
\hline & & & Pre2 & -.754 & -.362 \\
\hline & & & Pre3 & -.270 & -1.031 \\
\hline & & & Pre4 & .074 & -.836 \\
\hline & & & Pre5 & .120 & -1.168 \\
\hline & & & Pre6 & -.175 & -.644 \\
\hline & & & Pre7 & -.557 & -.524 \\
\hline & & & Pre8 & -.273 & -1.019 \\
\hline & & & Pre9 & -.252 & -.720 \\
\hline
\end{tabular}

See explanations of acronyms for items in Appendix 4. 
Appendix 6. Harman's Single Factor Test

\begin{tabular}{cccc}
\hline Component & Total & \% of Variance & Cumulative \% \\
\hline 1 & 3.94 & 35.84 & 35.84 \\
2 & 1.62 & 14.69 & 50.53 \\
3 & 1.22 & 11.09 & 61.62 \\
4 & .90 & 8.21 & 69.83 \\
5 & .86 & 7.84 & 77.67 \\
6 & .67 & 6.05 & 83.72 \\
7 & .60 & 5.48 & 89.20 \\
8 & .46 & 4.20 & 93.40 \\
9 & .37 & 3.39 & 96.79 \\
10 & .22 & 1.96 & 98.75 \\
11 & .14 & 1.25 & 100.00 \\
\hline
\end{tabular}

Appendix 7. Marker Variable Correlations

\begin{tabular}{|c|c|c|c|c|c|c|c|c|c|c|}
\hline Constructs & 1 & 2 & 3 & 4 & 5 & 6 & 7 & 8 & 9 & 10 \\
\hline 1. EAp & - & & & & & & & & & \\
\hline 2. $E A r$ & $.56^{\star \star \star}$ & - & & & & & & & & \\
\hline 3. PCO & $.29^{* * *}$ & $.39^{* * *}$ & - & & & & & & & \\
\hline 4. $M C$ & $.37^{\star \star \star}$ & $.39^{\star \star *}$ & $.29^{\star \star \star}$ & - & & & & & & \\
\hline 5. IndSC & .06 & .10 & .10 & .10 & - & & & & & \\
\hline 6. IntSC & $.24^{* * *}$ & $.17^{\star \star}$ & $.48^{* * *}$ & .11 & $.36^{* * *}$ & - & & & & \\
\hline 7. Marker & $.24^{\star \star \star}$ & $.33^{* * *}$ & $.19^{*}$ & $.17^{\star \star}$ & $.13^{*}$ & $.13^{*}$ & - & & & \\
\hline 8. ProRF & $.13^{*}$ & .05 & .00 & .06 & $.55^{\star * *}$ & $.43^{\star * *}$ & .03 & - & & \\
\hline 9. PreRF & $.19^{\star \star}$ & $.16^{*}$ & $.33^{\star \star \star}$ & .13 & -.10 & $.26^{\star \star \star}$ & .01 & .09 & - & \\
\hline 10. $\mathrm{CR}$ & $.40^{* * *}$ & $.33^{* * *}$ & $.17^{\star *}$ & $.39^{* * *}$ & .08 & .11 & .08 & .08 & $.16^{*}$ & - \\
\hline
\end{tabular}

$* p<.05, * * p<.01, * * * p<.001$; see List of Abbreviations for explanations of acronyms. 
Appendix 8. Two Waves of Respondents Key Variable Statistics

\begin{tabular}{|c|c|c|c|c|c|}
\hline \multirow{2}{*}{ Constructs } & \multirow{2}{*}{ Dimensions } & \multicolumn{2}{|c|}{ Before $(n=51)$} & \multicolumn{2}{|c|}{ After $(n=51)$} \\
\hline & & Mean $^{*}$ & $S D$ & Mean* & $S D$ \\
\hline \multirow{2}{*}{ EAp } & GAp & 3.86 & 1.721 & 3.80 & 1.822 \\
\hline & SAp & 3.98 & 1.903 & 4.04 & 1.697 \\
\hline \multirow{2}{*}{ EAr } & $\mathrm{GAr}$ & 2.77 & 1.416 & 2.97 & 1.619 \\
\hline & SAr & 2.57 & 1.330 & 2.49 & 1.188 \\
\hline CR & & 2.07 & .957 & 1.83 & .945 \\
\hline MC & & 3.46 & 1.428 & 3.40 & 1.517 \\
\hline BDI & & 4.57 & 2.265 & 4.43 & 2.394 \\
\hline AAt & & 3.12 & 1.501 & 3.37 & 1.480 \\
\hline MAt & & 3.50 & 1.625 & 3.80 & 1.364 \\
\hline \multirow{2}{*}{ sc } & IndSC & 4.95 & 1.026 & 4.63 & 1.054 \\
\hline & IntSC & 4.67 & 1.017 & 4.53 & .850 \\
\hline \multirow{2}{*}{ RF } & ProRF & 5.46 & 1.145 & 5.27 & 1.272 \\
\hline & PreRF & 4.26 & 1.227 & 4.49 & 1.142 \\
\hline PCO & & 4.05 & 1.350 & 3.92 & 0.973 \\
\hline AUDIT & & 8.94 & 6.143 & 8.41 & 6.457 \\
\hline Variables & Dimensions & $\mathbf{N}^{*}$ & $\%$ & $N^{*}$ & $\%$ \\
\hline \multirow{2}{*}{ Risk level } & Non- or low-risk & 24 & 47.1 & 26 & 51.0 \\
\hline & At-risk or high-risk & 27 & 52.9 & 25 & 49.0 \\
\hline \multirow{2}{*}{ Gender } & Male & 19 & 37.3 & 12 & 23.5 \\
\hline & Female & 32 & 62.7 & 39 & 76.5 \\
\hline
\end{tabular}

$*$ significance at $p>.05$; see List of Abbreviations for explanations of acronyms. 


\section{Appendix 9. Items Removed from the Final Model}

\begin{tabular}{|c|c|}
\hline Codes & Items \\
\hline \multicolumn{2}{|c|}{ Message compliance (MC) } \\
\hline MC1 & I intend to behave in ways that are consistent with the message. \\
\hline \multicolumn{2}{|c|}{ Interdependent self-construal (IntSC) } \\
\hline Int4 & I would offer my seat on the bus to my professor. \\
\hline Int11 & If my brother or sister fails, I feel responsible. \\
\hline Int12 & Even when I strongly disagree with group members, I avoid an argument. \\
\hline \multicolumn{2}{|c|}{ Prevention focus (PreRF) } \\
\hline Pre1 & In general, I am focused on preventing negative events in my life. \\
\hline Pre9 & $\begin{array}{l}\text { I see myself as someone who is primarily striving to become the self I "ought" to be - to fulfil my } \\
\text { duties, responsibilities, and obligations. }\end{array}$ \\
\hline
\end{tabular}

Appendix 10. Correlation Coefficients in the Modified Measurement Model

\begin{tabular}{cccc}
\hline \multicolumn{3}{c}{ Correlations } & Estimate \\
\hline \multicolumn{2}{c}{ Personal cultural orientation (PCO) } \\
ePCo3 & $<-->$ & ePCo4 & .343 \\
ePCo5 & $<-->$ & ePCo6 & .145 \\
\hline
\end{tabular}




\section{Appendix 11. Final Overall Measurement Model for Guilt}

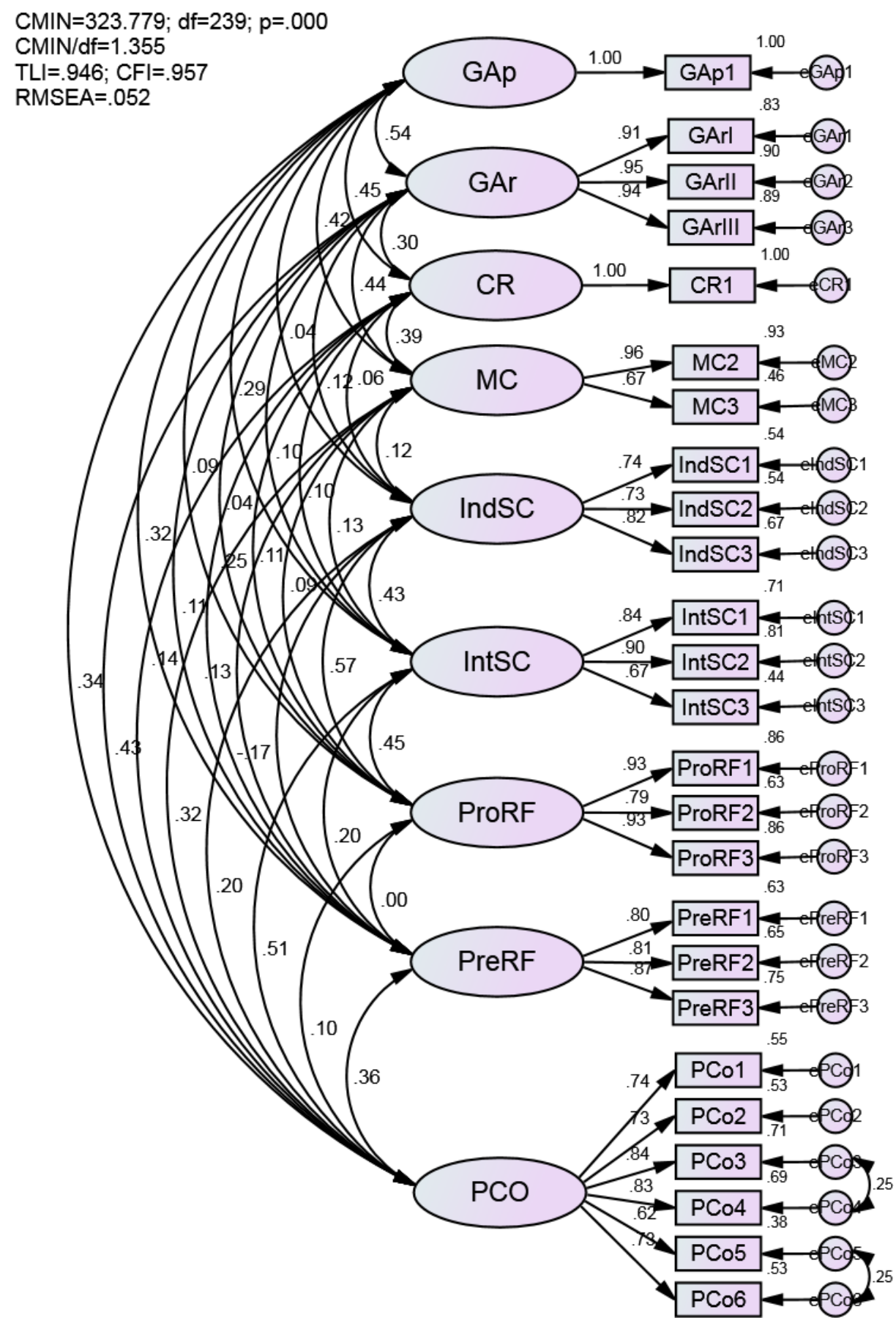


Appendix 12. Final Overall Measurement Model for Shame

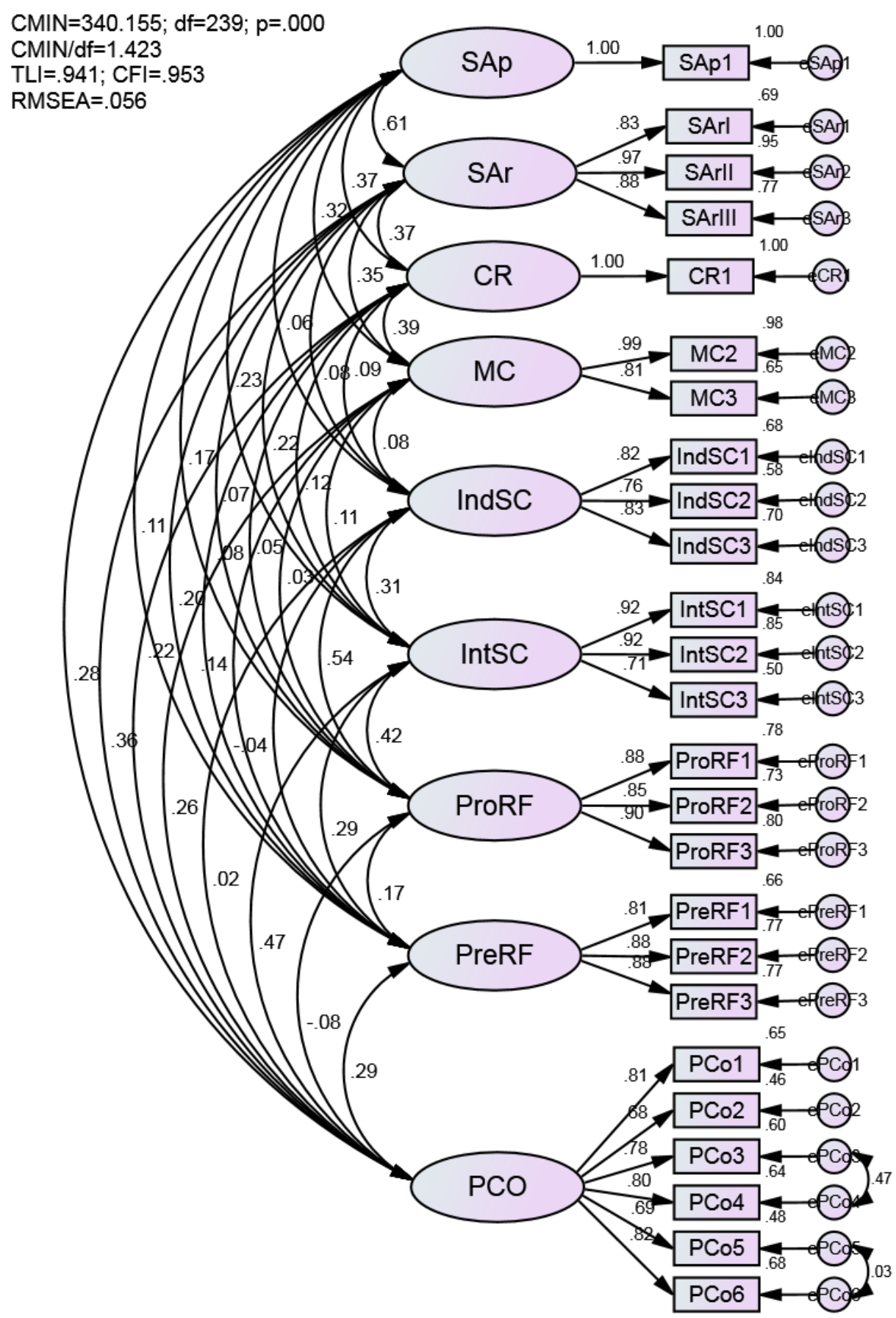

